\title{
Biochemical analysis of CYP74-enzymes in Physcomitrella patens
}

\author{
Dissertation \\ for the award of the degree \\ „Doctor rerum naturalium" \\ of the Georg-August-University Göttingen
}

submitted by

Julia Christine Scholz

from Kassel

Göttingen, 2013 
Member of the Thesis Committee (Reviewer): Prof. Dr. Ivo Feußner

Department of Plant Biochemistry, Albrecht-von-Haller-Institute, Georg-August-University Göttingen

Member of the Thesis Committee (Reviewer): $\quad$ Prof. Dr. Kai Tittmann

Department of Bioanalytics, Albrecht-von-Haller-Institute, Georg-August-University Göttingen

Member of the Thesis Committee: Prof. Dr. Marina Bennati

Electron Paramagnetic Resonance Spectroscopy Group, Max Planck Institute for Biophysical Chemistry, Göttingen

Date of oral examination: 


\section{Affidavit}

Herewith I declare that I wrote the PhD thesis on my own and without other sources and aids than quoted.

Julia Christine Scholz

Göttingen, February 2013 


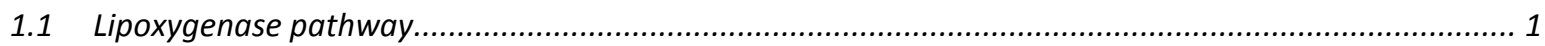

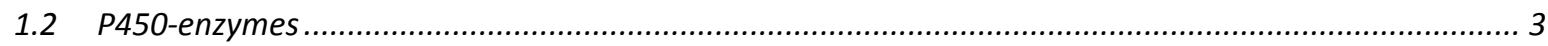

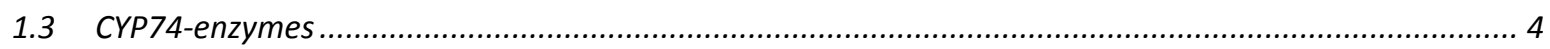

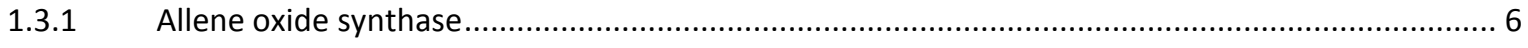

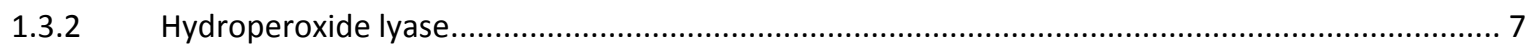

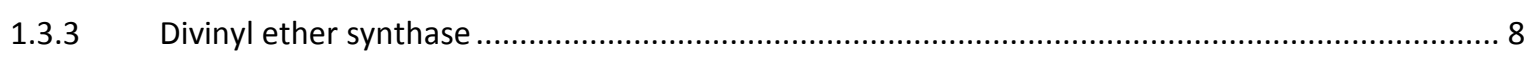

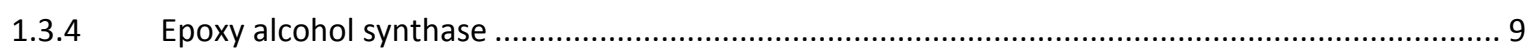

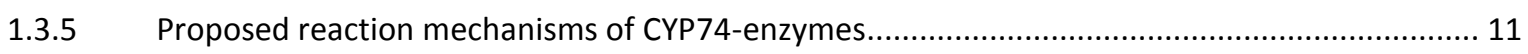

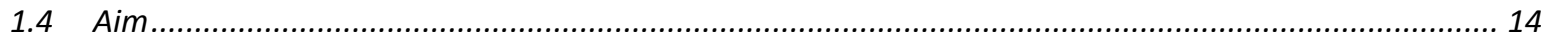

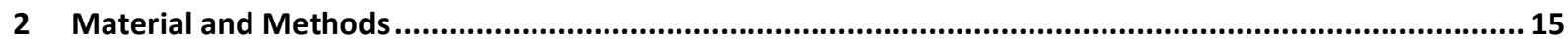

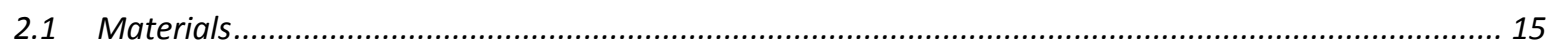

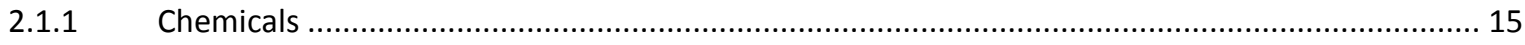

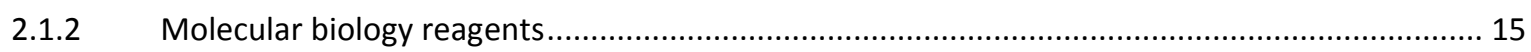

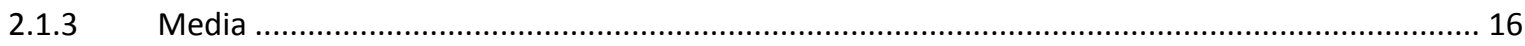

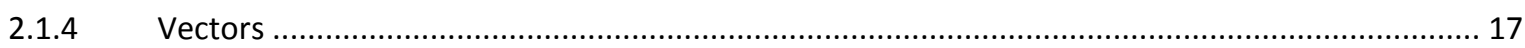

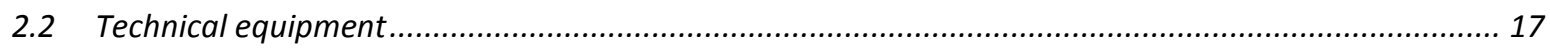

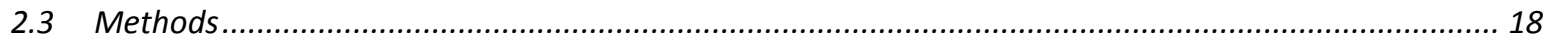

2.3.1 Amplification of specific DNA fragments by polymerase chainreaction (PCR) ......................... 18

2.3.1.1 Site-directed mutagenesis ...................................................................................... 18

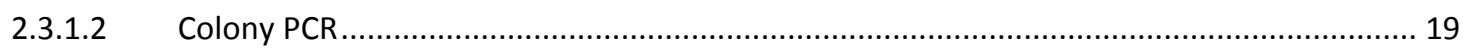

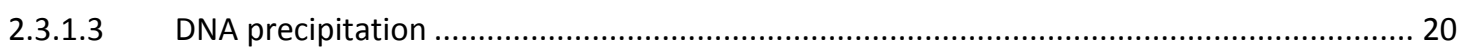

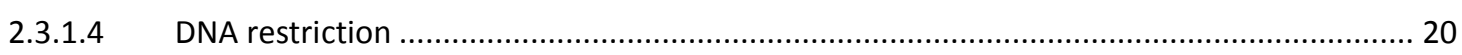

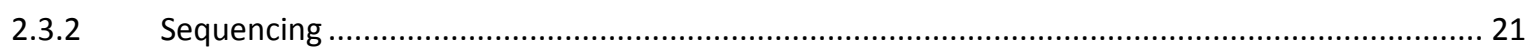

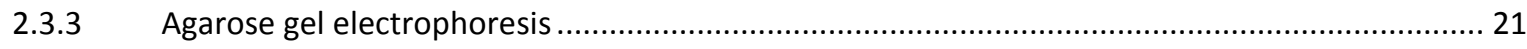

2.3.3.1 Separation of DNA fragments by agarose gel electrophoresis ...................................... 21

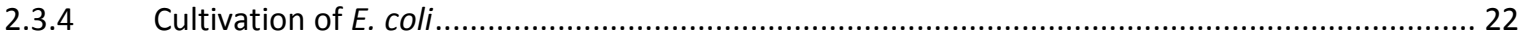

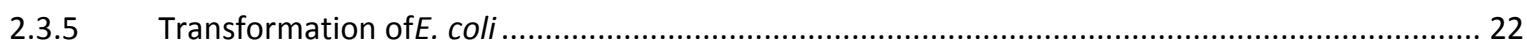

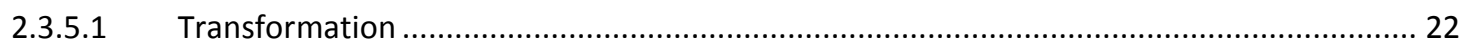

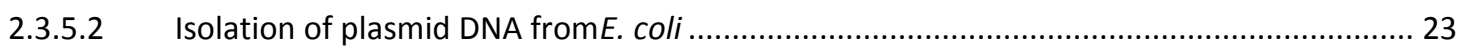

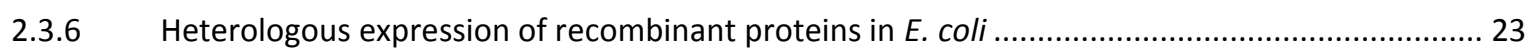

2.3.7 Cell disruption of E. coli expression cultures (adapted from (Richardson et al., 1993)) .............. 23

2.3.8 Purification of recombinant expressed proteins ...................................................................... 24

2.3.8.1 ImmobilizedMetal Ion Affinity Chromatography IMAC ............................................... 24

2.3.9 Sodium Dodecyl Sulfate PolyAcrylamide Gel Electrophoresis (SDS-PAGE) ..................................25

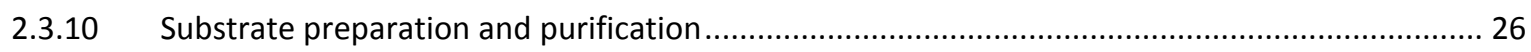

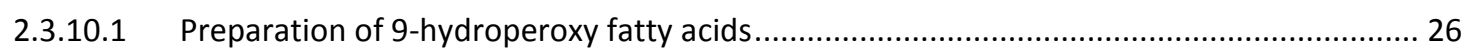

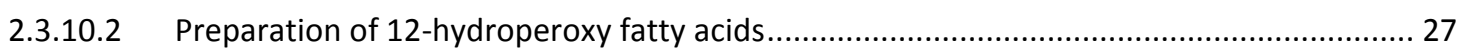

2.3.10.3 Preparation of 13- and 17-hydroperoxy fatty acids................................................. 27

2.3.10.4 Preparation of radio-labeled 9-and 13-hydroperoxy fatty acids ..................................... 27 


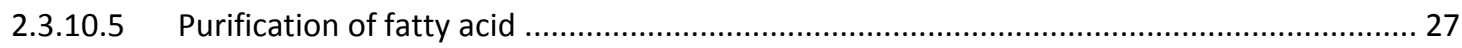

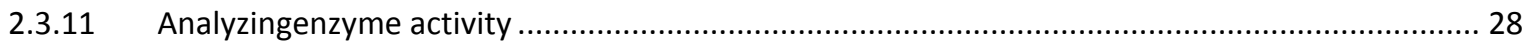

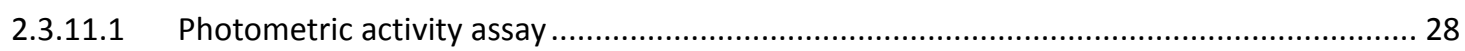

2.3.12 Determination of protein concentration with Bradford ....................................................... 29

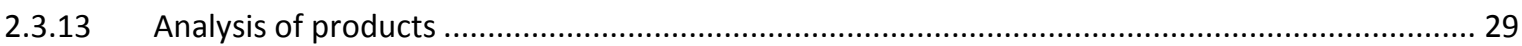

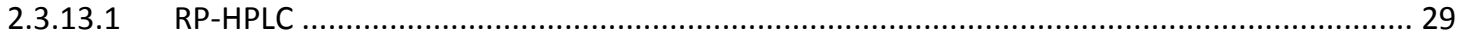

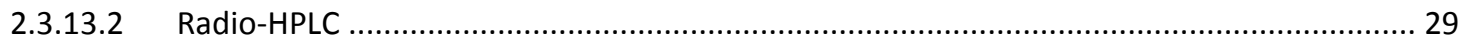

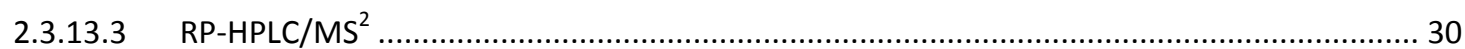

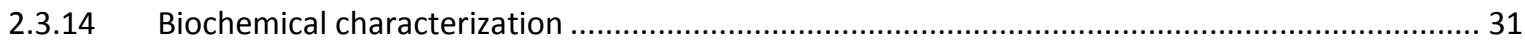

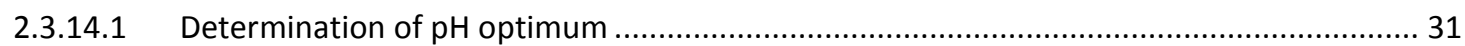

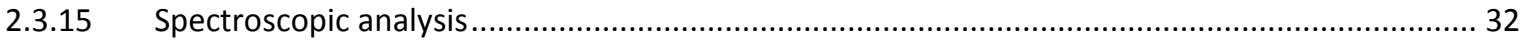

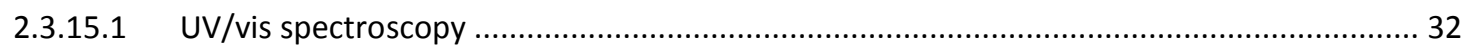

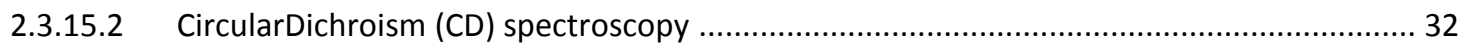

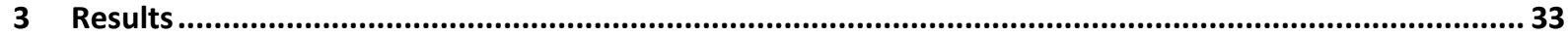

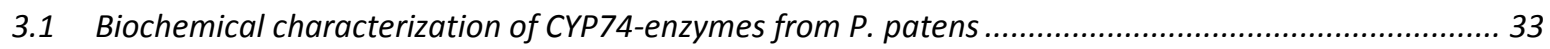

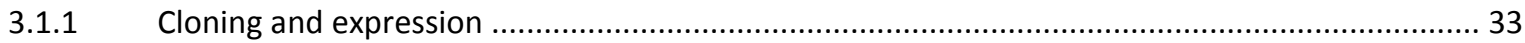

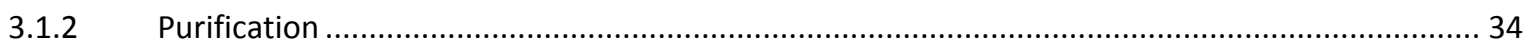

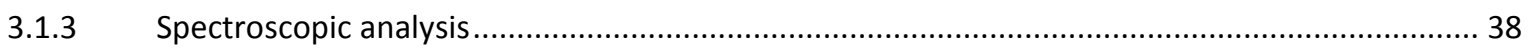

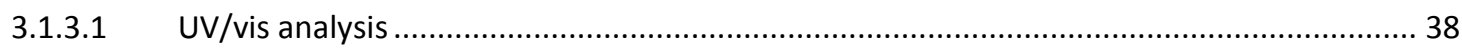

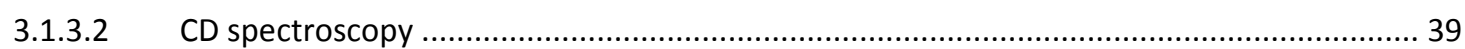

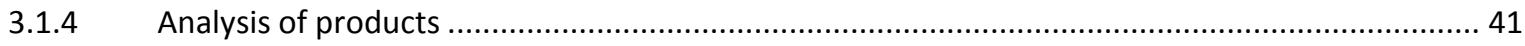

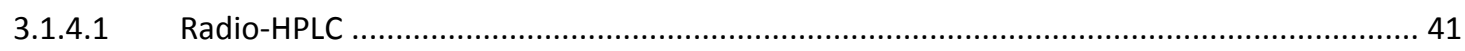

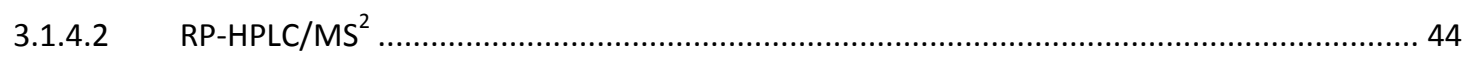

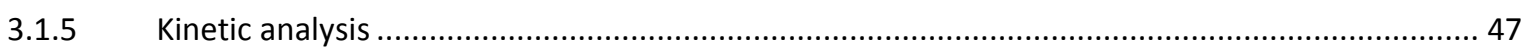

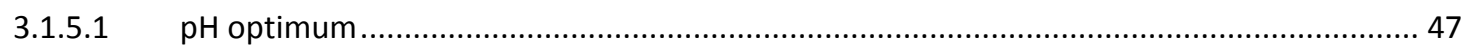

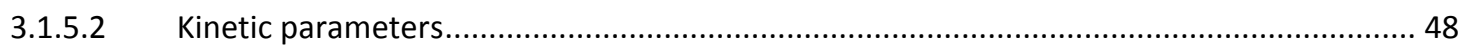

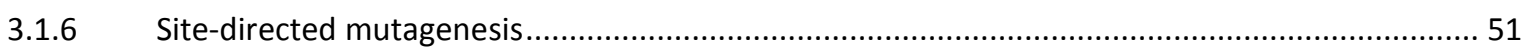

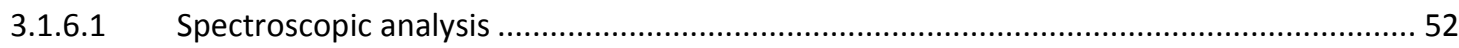

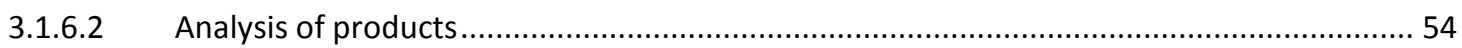

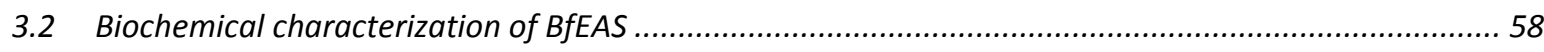

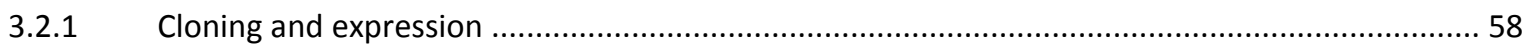

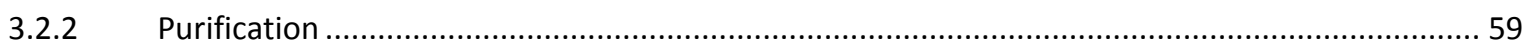

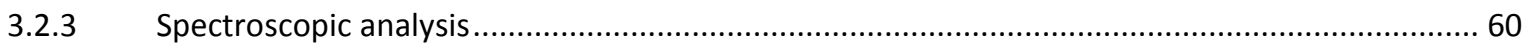

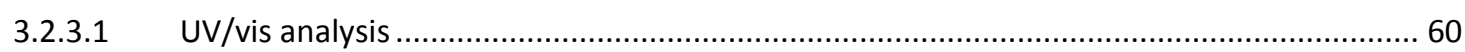

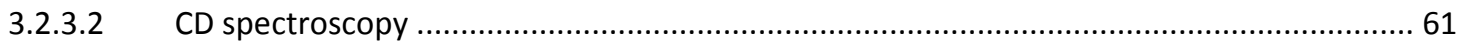

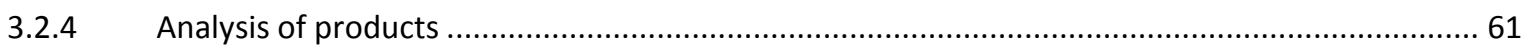

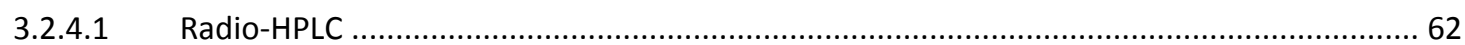

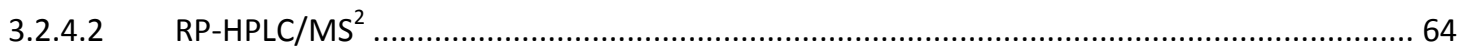

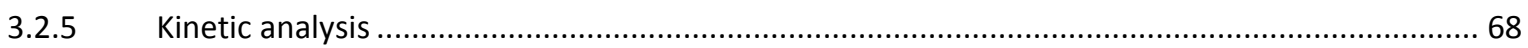

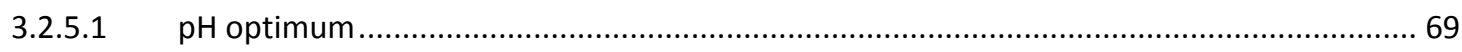

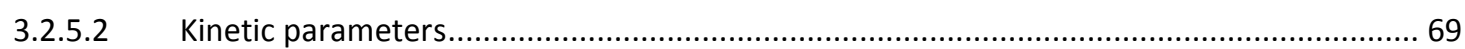

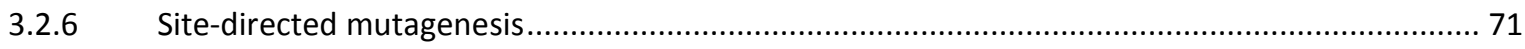

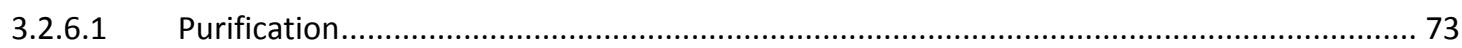




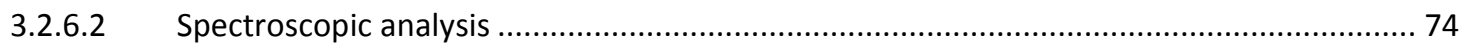

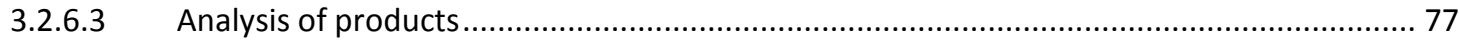

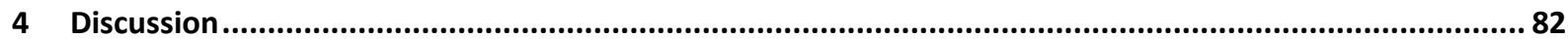

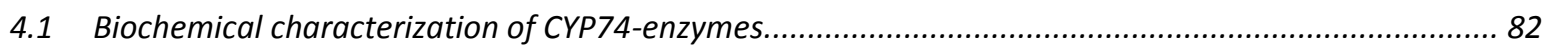

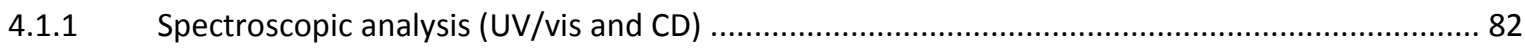

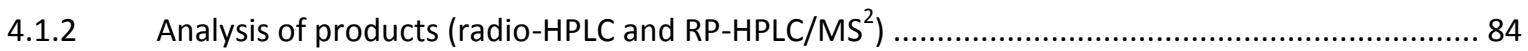

4.1.3 Kinetic analysis (pH optimum and kinetic parameters) ........................................................ 85

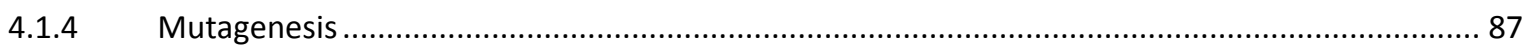

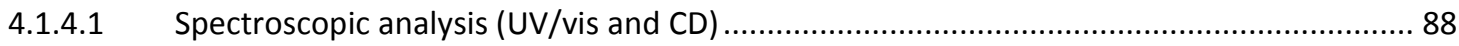

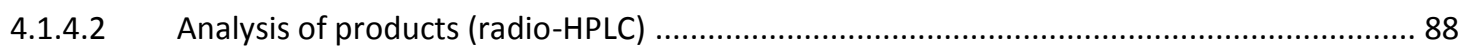

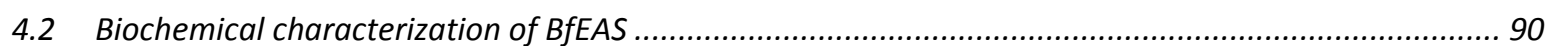

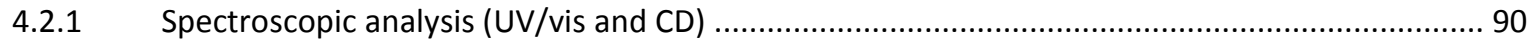

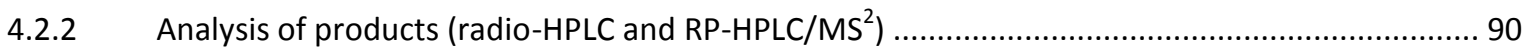

4.2.3 Kinetic analysis (pH optimum and kinetic parameters) ........................................................ 91

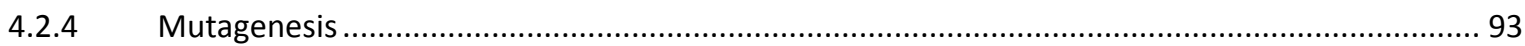

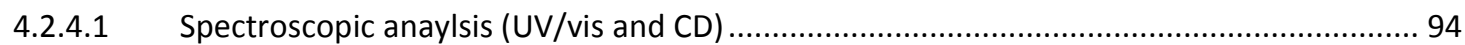

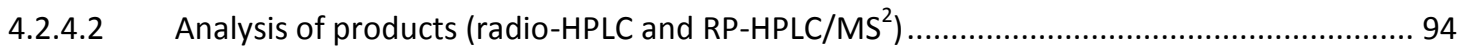

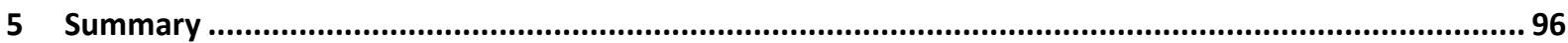

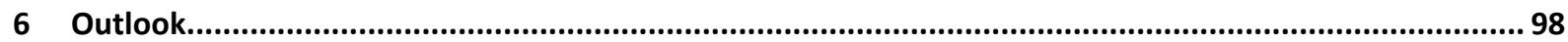

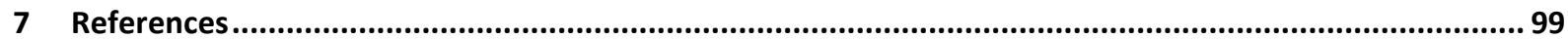

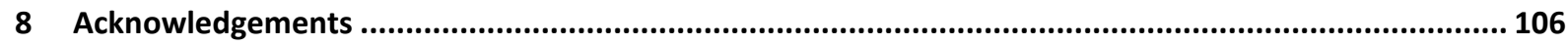

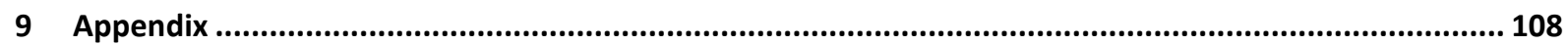

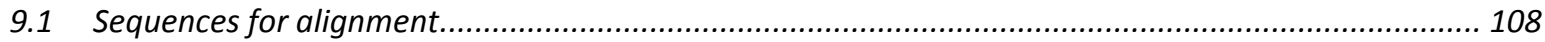

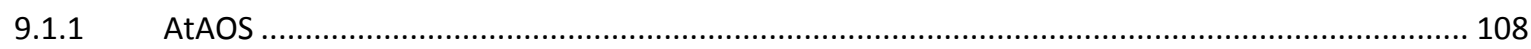

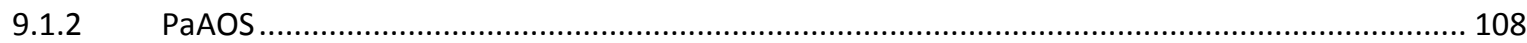

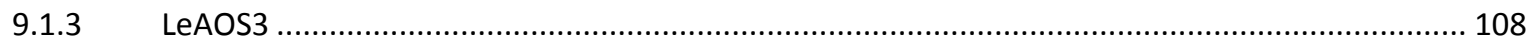

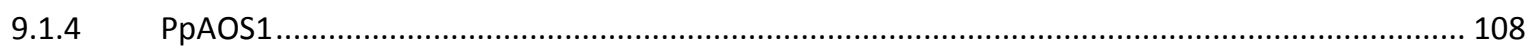

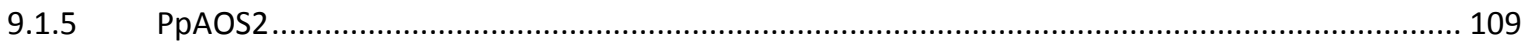

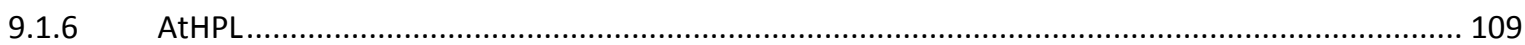

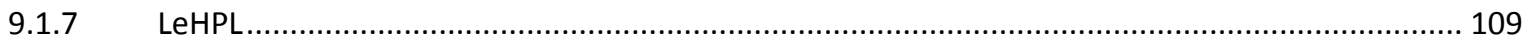

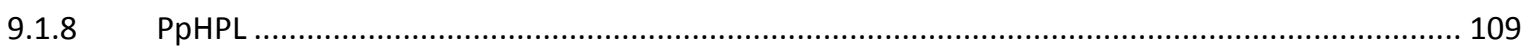

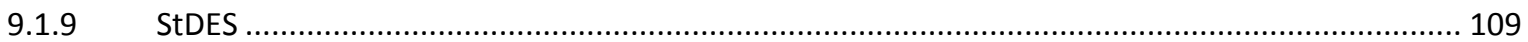

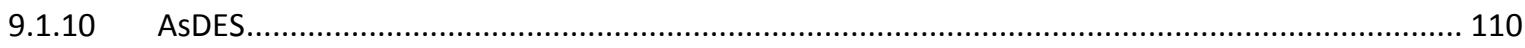

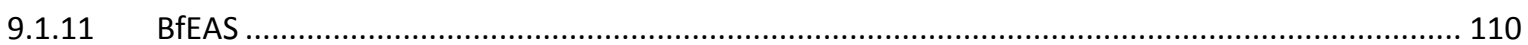

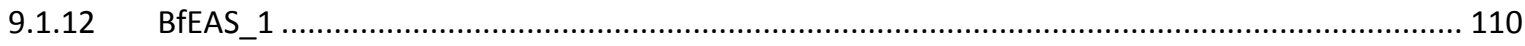

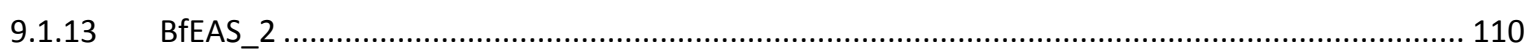

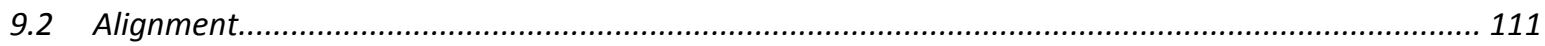

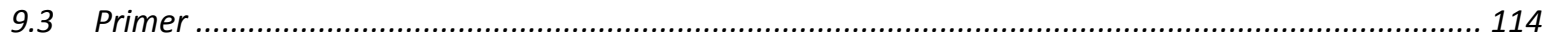




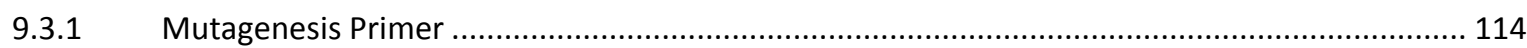

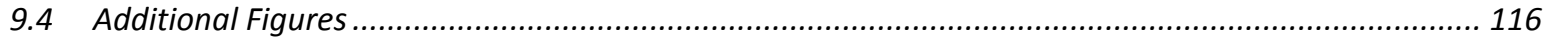

9.4.1 Biochemical characterization of CYP74-enzymes from $P$. patens ......................................... 116

9.4.1.1 Analysis of products(wild types) .......................................................................... 116

9.4.1.2 Analysis of products (site-directed mutagenesis) .................................................... 116

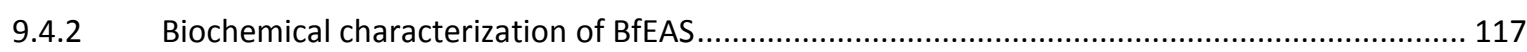

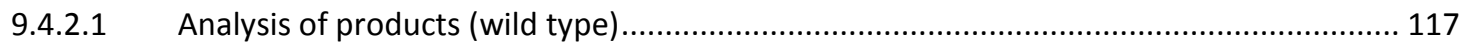

9.4.2.2 Analysis of products (site-directed mutagenesis) ..................................................... 117

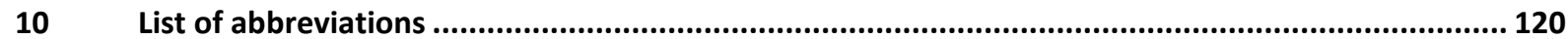

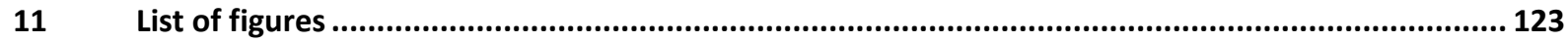

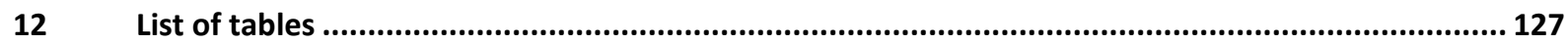

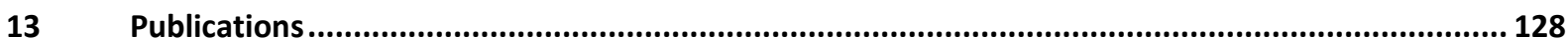




\section{Introduction}

\subsection{Lipoxygenase pathway}

Lipoxygenases (LOX) are widely spread in higher organisms and were found in fungi (Tsitsigiannis and Keller, 2007), mammals (Funk, 2001) and flowering plants (Liavonchanka and Feussner, 2006). They were also found in bacteria (Iny et al., 1993, Porta and Rocha-Sosa, 2001, Lang et al., 2008) and in non-flowering plants like the moss Physcomitrella patens(Senger et al., 2005).

LOXs catalyze the first committed step in the biosynthesis of oxylipins which function as signaling molecules. In fungi LOX-derived oxylipins are involved in the regulation of developmental stages and pathogenesis (Noverr et al., 2003), whereas in mammals the LOX-derived oxylipins (e.g. leukotrienes) are involved in the regulation of inflammatory processes (Funk, 2001). In plants they are involved in developmental processes, in wound response and in pathogen defense reactions (Stumpe et al., 2005, Howe and Jander, 2008,Wasternack, 2007). In non-flowering plants oxylipins are involved in developmental processes (Stumpe et al., 2010).The biological function in prokaryotes is still scarce (Andreou et al., 2009).

LOXs belong to the dioxygenase family and contain a non-heme iron in its active site. They catalyze the formation of $(1 Z, 3 E)$-hydroperoxy fatty acids by insertion of molecular oxygen in the $(1 Z, 4 Z)$ pentadiene system of polyunsaturated fatty acids (PUFAs). Plants use the $C_{18}$ fatty acids linoleic acid

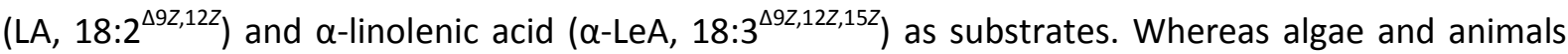
use additionally to the $C_{18}$ fatty acids the $C_{20}$ fatty acids arachidonic acid (ARA, 20:4 $4^{\Delta 5 z, 8 z, 11 z, 14 z}$ ) and eicosapentaenoic acid (EPA, 20:5 $5^{\Delta 5 z, 8 z, 11 z, 14 z, 17 z}$ ) (Wichard et al., 2005, Andreou et al., 2009).

Considering LA or $\alpha$-LeA as substrates for LOX, introduction of the hydroperoxide group can occur either at position $C_{9}$ (9-LOX) or at position $C_{13}$ (13-LOX) of the $C_{18}$ fatty acid, therefore LOXs can be divided into 9-LOX and 13-LOX. 9-LOX leads to the (9S)-hydroperoxide derivatives (9S,10E,12Z)-9hydroperoxy-10,12-octadecadienoic acid (9-HPOD) or (9S,10E,12Z,15Z)-9-hydroperoxy-10,12,15octadecatrienoic acid (9-HPOT), respectively, and 13-LOX to (13S)-hydroperoxide derivatives (9Z,11E,13S)-13-hydroperoxy-9,11-octadecadienoic acid (13-HPOD) or (9Z,11E,13S,15Z)-13hydroperoxy-9,11,15-octadecatrienoic acid (13-HPOT), respectively (Liavonchanka and Feussner, 2006, Feussner and Wasternack, 2002).

In Figure 1.1 the conversion of $C_{18}$ fatty acid linoleic acid (LA) by 9- and 13-LOX to 9-HPOD and 13HPOD, respectively, is shown. 


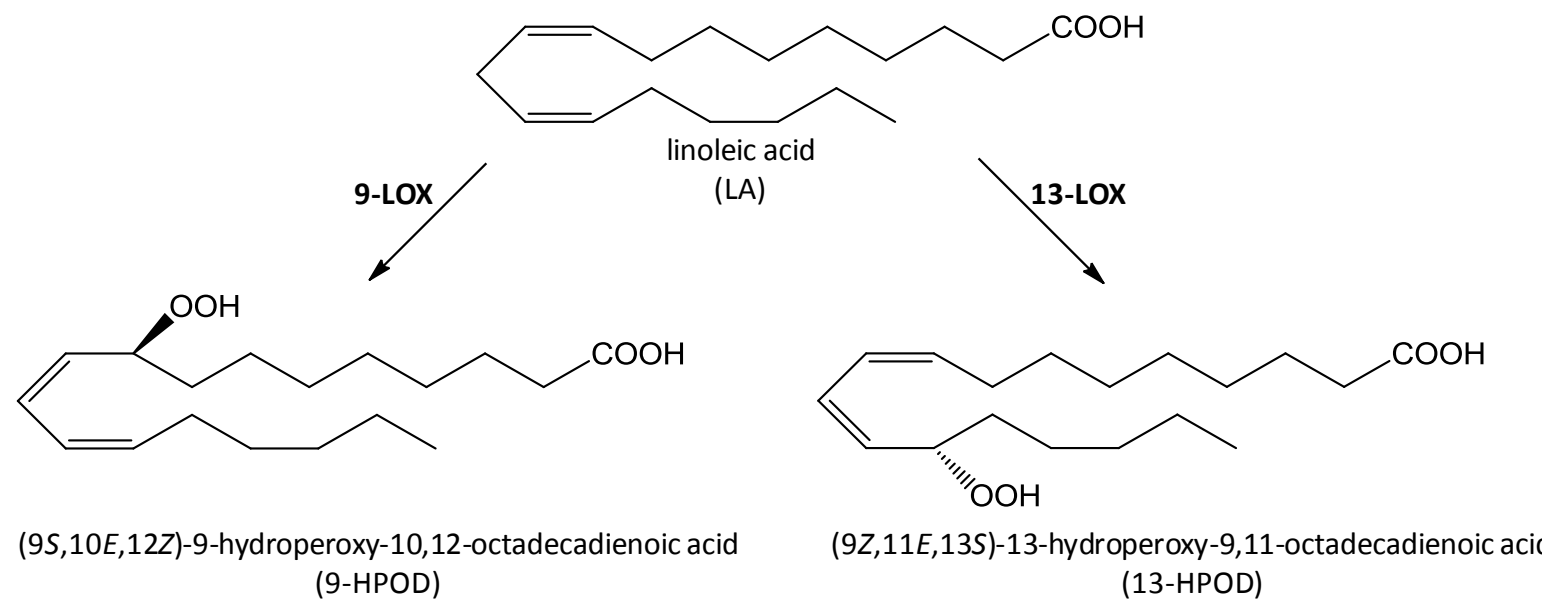

Figure 1.1: Synthesis of hydroperoxides derived from LA by 9-LOX or 13-LOX (adapted from (Stumpe and Feussner, 2006)). LOX introduce molecular oxygen into the fatty acid either at position $\mathrm{C}_{9}$ or position $\mathrm{C}_{13}$ to generate hydroperoxy fatty acids.

LOX (Figure 1.2) generated fatty acid hydroperoxides can subsequently be metabolized by further enzymes, like the allene oxide synthase (AOS), hydroperoxide lyase (HPL), divinyl ether synthase (DES), epoxy alcohol synthase (EAS), peroxygenase (PXG), and by LOX itself and lead to various products known as oxylipins (Hughes et al., 2009). Oxylipins are fatty acid hydroperoxides, hydroxy-, epoxy-, keto- and oxo-fatty acids, epoxy alcohols, divinyl ethers, volatile alcohols and aldehydes, jasmonic acid and their derivatives (Mosblech et al., 2009).

AOS, HPL, DES and EAS contribute to a special enzyme family which is described later in this chapter (see 1.3). PXG converts the hydroperoxides to epoxy-, epoxyhydroxy-, dihydroxy- and polyhydroxy fatty acids (Feussner and Wasternack, 1998). Reduction of hydroperoxy fatty acids to hydroxyl derivatives can occur in addition via a non-enzymatical or chemical process. Under anaerobic condition homolytic cleavage of the oxygen binding occurs through the LOX itself, which leads to the formation of ketodienes (KOD) and ketotrienes (KOT) (Feussner and Wasternack, 2002). 


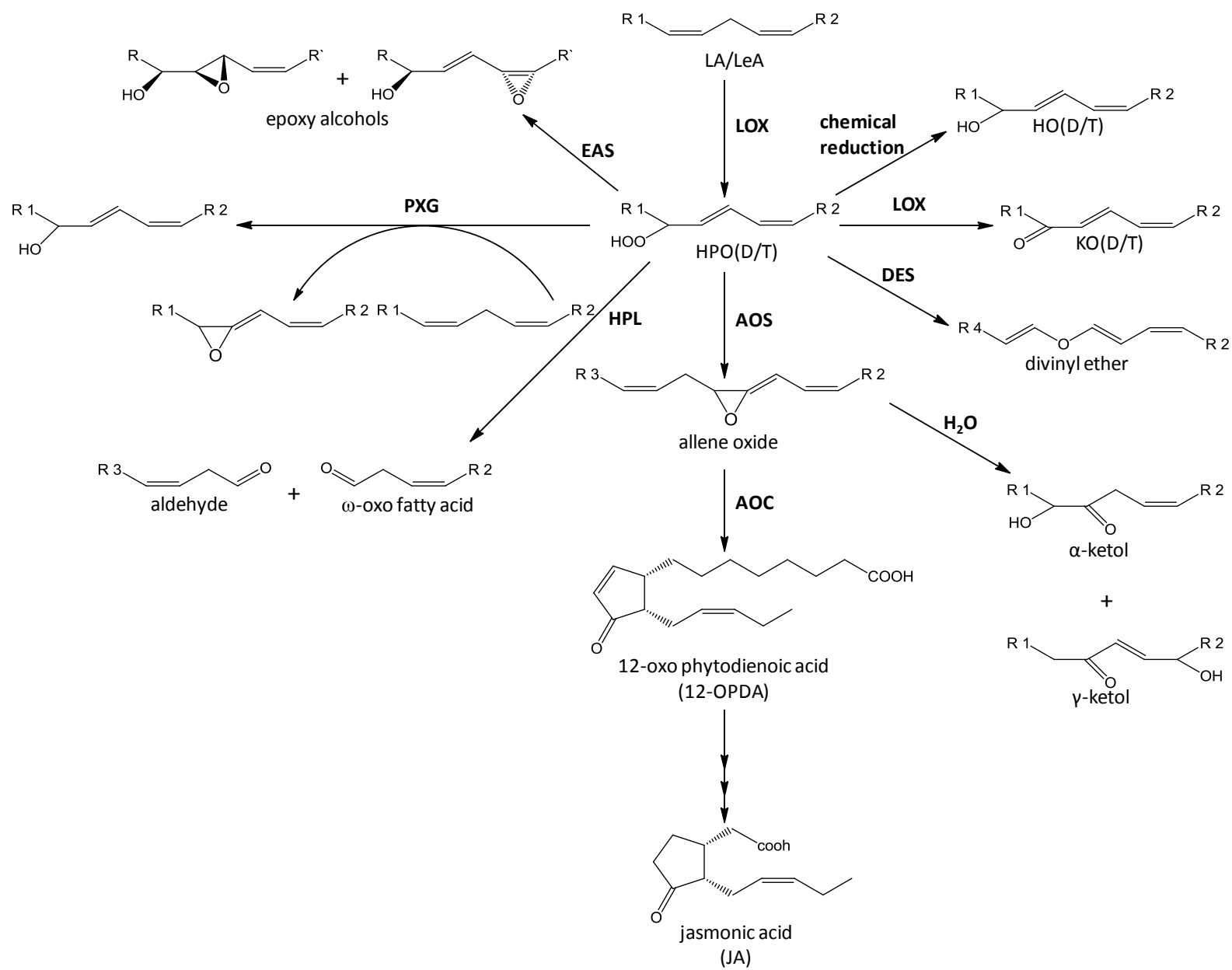

Figure 1.2: Formation of oxylipins in plants (adapted from (Göbel et al., 2001)). The insertion of molecular oxygen into polyunsaturated fatty acids (PUFAs) like linoleic (LA) or linolenic acid (LeA) by lipoxygenases (LOXs) generates hydroperoxy fatty acids which can be further metabolized to oxylipins by various enzymes. Abbreviations: HPO(D/T): hydroperoxy octadecadienoic/octadecatrienoic acid, EAS: epoxy alcohol synthase, $\mathrm{HO}(\mathrm{D} / \mathrm{T})$ : hydroxyl octadecadienoic/octadecatrienoic acid, PXG: peroxygenase, KO(D/T): keto octadecadienoic/octadecatrienoic acid, HPL: hydroperoxide lyase, AOS: allene oxide synthase, DES: divinyl ether synthase, AOC: allene oxide cyclase.

\subsection{P450-enzymes}

Cytochrome P450-enzymes (CYP) build one of the largest super families of enzymes. They are found in all biological organisms and catalyze a variety of chemical reactions in mammals and plants (Denisov et al., 2005).

P450-enzymes are heme containing enzymes and the prosthetic heme iron is bound to a cysteine residue as fifth ligand to the heme iron(Werck-Reichhart and Feyereisen, 2000).

Their name originates from the observation that in complex with carbon monoxide P450-enzymes have a characteristic absorption maximum at $450 \mathrm{~nm}$ (Denisov et al., 2005, Marnett, 2008). Enzymes of the CYP74-family also belong to the CYPs (see 1.3).

Most P450-enzymes act as monooxygenases, they need molecular oxygen and a NAD(P)H-dependent reductase as co-substrates. 


\subsection{CYP74-enzymes}

CYP74-enzymes build a subgroup of the CYP-superfamily described before (see 1.2). AOS, HPL, DES, and EAS belong to the cytochrome P450 subfamily. In contrast to most P450-enzymes the CYP74enzymes are not functioning as monooxygenases but rather as hydroperoxide isomerases (HPL) or dehydratases (AOS and DES) (Stumpe and Feussner, 2006, Lee et al., 2008,Toporkova et al., 2008). CYP74-enzymes do not need molecular oxygen or NAD(P)H-dependent reductase as cofactors. They use acylhydroperoxides both as substrate and as oxygen donor to generate new carbon bonds by isomerization reactions (Noordermeer et al., 2001a, Andreou et al., 2009). Therefore the catalytic centers of CYP74s are very active (Hughes et al., 2006a). A further difference to the classical P450enzymes is that in the center of CYP74-enzymes heme-binding loop an additional nine amino acid residue insertion is found instead of the conserved threonine found in all other P450s(Marnett, 2008).

Functional differences of CYP74- and P450-catalysis is reflected by the different active site architectures of the enzymes which was demonstrated by 3D-structures of AOSs from Arabidopsis and guayule, which preclude monooxygenase-activity from unusual P450s (Lee et al., 2008, Li et al., 2008). Mechanic aspects of the relationship between structure and function are still not fully understood (Brash, 2009).

Based on sequence similarity and substrate specificity CYP74-enzymes are further divided into four subfamilies (see Figure 1.3). Within a subfamily the amino acid sequence has an identity of more than $55 \%$ to each other (Howe and Schilmiller, 2002). 


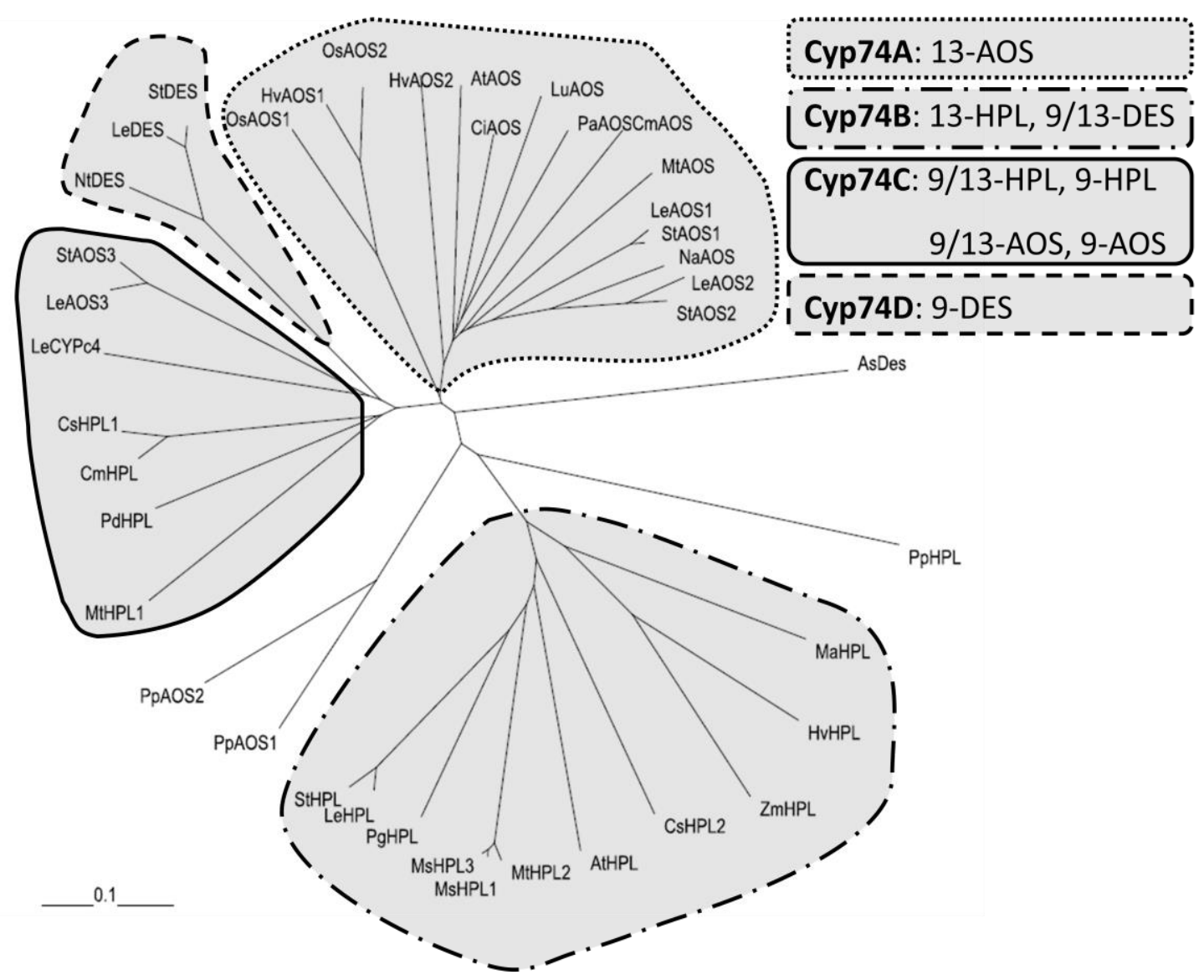

Figure 1.3: Phylogenetic analysis of different CYP74 enzymes from different plant species (taken from (Scholz et al., 2012)). The phylogenetic tree was calculated using the ClustalX software package employing default parameters. Allium sativum (As); Arabidopsis thaliana (At); Citrus sinensis (Ci); Cucumis melo (Cm); Cucumis sativum (Cs); Hordeum vulgare (Hv); Lycopersicum esculentum (Le); Musa ascuminata (Ma); Medicago sativum (Ms); Medicago truncatula (Mt); Nicotiana attentuata (Na); Nicotiana tabacum (Nt); Oryzae sativum (Os); Parthenium argentatum (Pa); Prunus dulcis (Pd); Psidium guajava (Pg); Physcomitrella patens (Pp); Solanum tuberosum (St); Zea mays (Zm). AtAOS, CAA63266; AsDES, AJ867809; AtHPL, AAC69871; CiAOS, AA072741; CmAOS, AAM66138; CmHPL, AAK54282; CsHPL1, AAF64041; CsHPL2, AF229812; HvAOS1, CAB86384; HvAOS2, CAB86383; HVHPL, CAC82980; LeAOS1, CAB88032; LeAOS2, AAF67141; LeAOS3, AAN76867; LeCYPC4, AAL86702; LeDES, AAG42261; LeHPL, AAF67142; LUAOS, AAA03353; MaHPL,CAB39331; MsHPL1, CAB54847; MsHPL2, CAB54848; MsHPL3, CAB54849; MtAOS, CAC86897; MtHPL2, CAC86899; MtHPL1,CAC86898; NaAOS, CAC82911; NtDES, AAL40900; OsAOS1, AY055775; OsAOS2, AAL38184; PaAOS, CAA55025; PdHPL, CAE18065; PgHPL, AAK15070; PpAOS1,CAC86919; PpAOS2, XP_001759629; PpHPL, CAC86920; StAOS1,CAD29735; StAOS2, CAD29736; StAOS3, CAI30876; StDES, CAC28152; StHPL, CAC44040; ZmHPL, AAS47027.

To CYP74A-subfamily belongs AOS with a preference for 13-hydroperoxides as substrate. 13-HPL and 13-DES build subfamily CYP74B. HPL with a preference for 9- and 13-hydroperoxides as substrate, and HPL which use only 9-hydroperoxides as substrate, form the CYP74C-family. 9/13- and a 9-AOS also belong to this family. Enzymes with 9-DES activity are found in the CYP74D-family(Stumpe and Feussner, 2006, Gogolev et al., 2011).

While the cDNAs of the CYP74-enzymes AOS, DES and HPL were identified in plants over the last ten years, in 2008 a fourth CYP74-enzyme EAS was isolated from an animal (Lee et al., 2008). 


\subsubsection{Allene oxide synthase}

The first described CYP74-enzyme was the AOS from flax (Linum usitatissimum) (Song and Brash, 1991). AOS is a key-enzyme in the biosynthesis of the phytohormone jasmonic acid (JA) and is therefore involved in signal and defense responses in higher plants and algae (Andreou et al., 2009). It was first described as hydroperoxide isomerase or hydroperoxide dehydratase (Hamberg and Gardner, 1992). The AOS-branch of the oxylipin pathway takes place in the plastid. AOS catalyzes the conversion of a fatty acid hydroperoxide into a highly unstable allene oxide with a life time of about $30 \mathrm{~s}$, which then is subsequently hydrolyzed into $\alpha$-ketol and $y$-ketol as well as into cyclopentenone derivatives (Brash et al., 1988, Hamberg, 1997, Hamberg, 1987). In Figure 1.4 the reaction for the conversion of 13-HPOT by AOS is shown. The 13-HPOT is dehydrated to the unstable allene oxide (9Z,11E,13S,15Z)-12,13-epoxy-9,11,15-octadecatrienoic acid (12,13S-EOT). The 12,13S-EOT is than hydrolyzed into $\alpha$-ketol $(9 Z, 15 Z)-12-o x o-13-h y d r o x y-9,15$-octadecadienoic acid and $\psi$-ketol $(10 E, 15 Z)$ 12-oxo-9-hydroxy-10,15-octadecadienoic acid or cyclized to racemic (9S,10Z,13S,15Z)-12-oxo-10,15phytodienoic acid (12-OPDA) (Brash et al., 1988). Chemical cyclization leads to the formation of racemic OPDA, whereas cyclization by allene oxide cyclase $(A O C)$ arises optically pure $(9 S, 13 S)$-12OPDA (Hamberg and Fahlstadius, 1990). The reduction of $(9 S, 13 S)$-12-OPDA by OPDA reductase isoform 3 (OPR3) and three cycles of $\beta$-oxidation leads to the formation of JA and takes place in the peroxisome (Ziegler et al., 1999, Grechkin et al., 2008, Vick and Zimmerman, 1984, Itoh et al., 2002). The active form of $\mathrm{JA}$, its isoleucine conjugate, regulates developmental processes as well as defense responses (Howe and Jander, 2008, Wasternack, 2007).

All purified AOS are membrane bound. There were each one AOS described for Arabidopsis (Arabidopsis thaliana), flax, guayule (Partheniumargentatum) and alfalfa (Medicagotruncatula), two were found in barley (Hordeum vulgare) and three have been described for tomato (Lycopersicum esculentum) and potato (Solanum tuberosum) (Andreou et al., 2009, Stumpe and Feussner, 2006, Mosblech et al., 2009). 


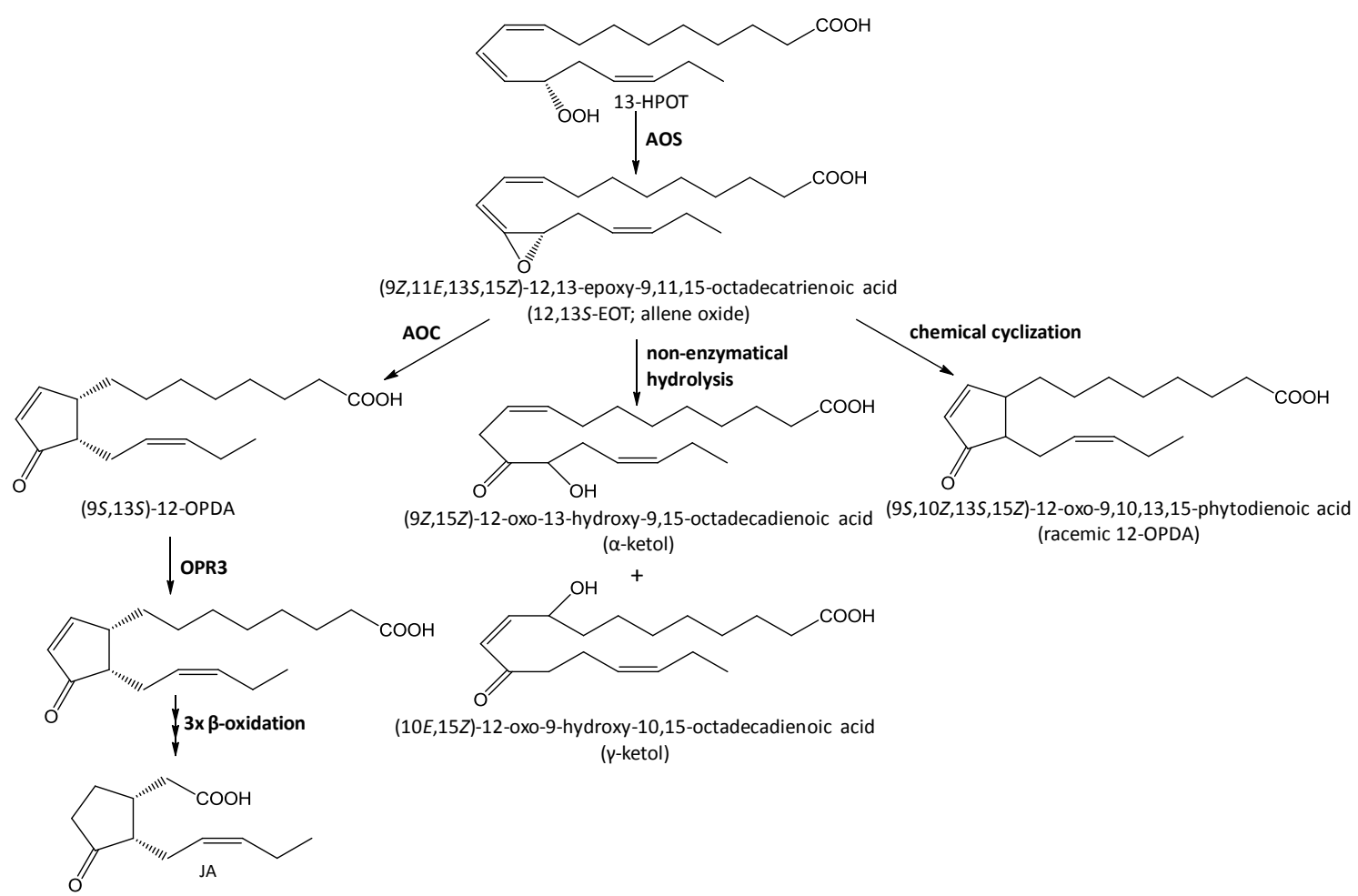

Figure 1.4: AOS pathway for the conversion of 13-HPOT (adapted from (Stumpe and Feussner, 2006)). The fatty acid hydroperoxide is converted by AOS into an unstable allene oxide, which is further hydrolyzed into $\alpha$-ketol and $y$-ketol. Chemical cyclization leads to the formation of racemic 12-OPDA. Cyclization by AOC arise enantiomeric pure 12-OPDA. The reduction by OPR3 and three cycles of $\beta$-oxidation leads to the formation of jasmonic acid. Abbreviations: 13-HPOT: 13-hydroperoxy octadecatrienoic acid, AOS: allene oxide synthase, AOC: allene oxide cyclase, 12-OPDA: (9S,10Z,13S,15Z)-12-oxo-10,15-phytodienoic acid, OPR3: OPDA reductase.

\subsubsection{Hydroperoxide lyase}

HPL catalyzes homolytic cleavage of fatty acid hydroperoxides in $\omega$-oxo fatty acids and short chain aldehydes (Andreou et al., 2009). HPL was first described as hydroperoxide isomerase and only recently its hemiacetal hydroperoxide lyase activity was unveiled (Grechkin and Hamberg, 2004).

In Figure 1.5 exemplarily the HPL pathway for 9- and 13-LOX derived fatty acid hydroperoxides from $\alpha$-LeA is shown. HPL converts the fatty acid hydroperoxide into an unstable hemiacetal, which spontaneously dissociates into short chain alkenals (Grechkin and Hamberg, 2004). The alkenals generated by $13-\mathrm{HPL}$ can be enzymatically isomerized from (3Z)- to $(2 E)$-enol formation and be reduced by alcohol dehydrogenase (ADH) to alcohols. The $\omega$-oxo fatty acids such as (9Z)-12-oxo-9dodecenoic acid can be isomerized to the wound hormone traumatin ((10E)-12-oxo-10-dodecenoic acid) which spontaneously can oxidize to traumatic acid (Noordermeer et al., 2000).

Green leaf volatiles (aldehydes and alcohols) derived from 13-LOX pathway have a chain length of six carbon atoms and are characteristic for the odor of vegetables, fruits and green leaves (Matsui et al., 1996). 
Oxylipins generated by HPL seem to be involved in plant defense processes (Howe and Jander, 2008) and in wound healing (Matsui et al., 2006).

HPL was isolated from A. thaliana, maize (Zea mays), barley, guava (Psidium guajava), bell pepper (Casicum anuum), melon (Citrullus lanatus), potato, and tomato (Matsui, 2006, Stumpe and Feussner, 2006, Mosblech et al., 2009, Matsui et al., 2006). Most HPLs have a preference for 13hydroperoxides as substrate, while HPL from melon and cucumber (Cucumis sativus) uses both 9and 13-hydroperoxides (Grechkin, 2002).

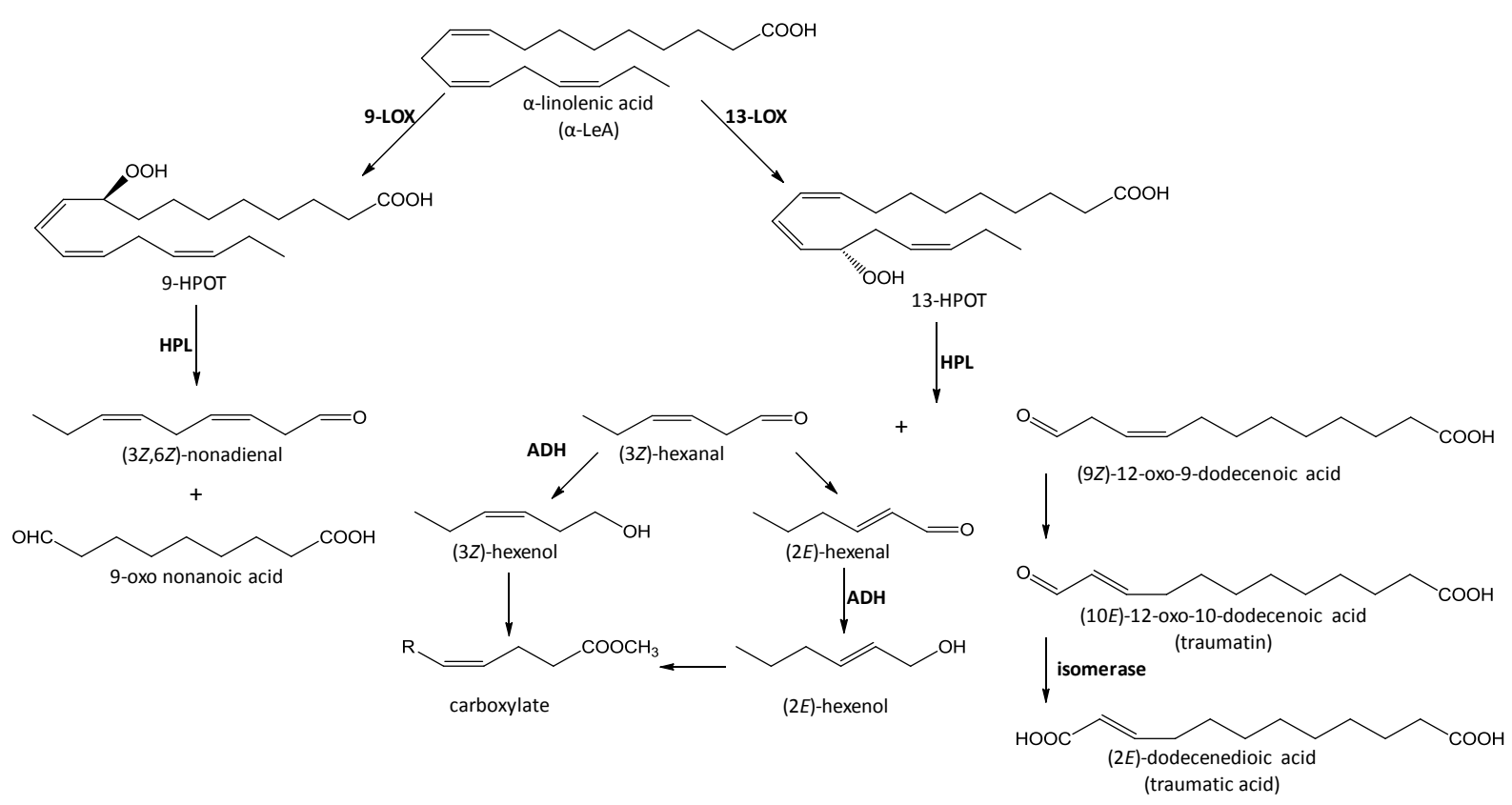

Figure 1.5: HPL pathway for the conversion of $\alpha$-LeA (adapted from (Stumpe and Feussner, 2006)). 9-HPOT and 13-HPOT derived from $\alpha$-LeA are converted by HPL into alkenals and $\omega$-oxo fatty acids. The alkenals can enzymatically be isomerized to enols and reduced to alcohols by $A D H$ as shown for the $C_{6}$ isomers. The $C_{12} \omega$ oxo fatty acid can isomerize to traumatin which can be oxidized to traumatic acid. Abbreviations: 9-LOX: 9lipoxygenase, 13-LOX: 13-lipoxygenase, 9-HPOT: 9-hydroperoxy octadecatrienoic acid, 13-HPOT: 13hydroperoxy octadecatrienoic acid, $\mathrm{ADH}$ : alcohol dehydrogenase.

\subsubsection{Divinyl ether synthase}

DES catalyzes the conversion of a fatty acid hydroperoxide derivative into divinyl ether (Stumpe et al., 2008). The conjugated divinyl ether fatty acid has molecular oxygen within its carbon chain (Itoh and Howe, 2001).

The divinyl ether colneleic acid (CA, 9-[(1' $\left.E), 3^{\prime} Z, 8 E\right)$-nonadienyloxy]-8-nonenoic acid) and colnelenic

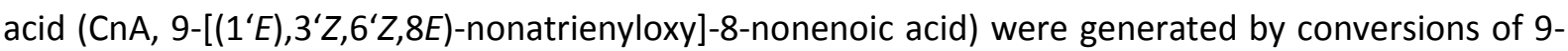
hydroperoxides derived from LA and $\alpha$-LeA, while etheroleic acid (EA, 12-[(1'E,9Z,11E)-hexenyloxy]9,11-dodecadienoic acid) and etherolenic acid (EnA, 12-[(1'E, $\left.3^{\prime} Z, 9 Z, 11 E\right)$-hexadienyloxy]-9,11dodecadienoic acid) arise by conversions of 13-hydroperoxides (Hamberg, 1998). Exemplarily in 
Figure 1.6 the DES pathway for the conversion of 9- and 13-hydroperoxides derived from $\alpha$-LeA is shown.

In tomato, tobacco (Nicotiana tabacum) and potato 9-hydroperoxide specific cDNAs were isolated (Fammartino et al., 2007, Stumpe et al., 2001,Itoh and Howe, 2001). In garlic (Allium sativum) DES with preference for both 9- and 13-hydroperoxides (9/13-DES) was isolated (Stumpe et al., 2008, Chechetkin et al., 2008). DES activity was detected in Lily-of-the-Valley (Convallaria majalis) and in flax (Stumpe et al., 2008, Chechetkin et al., 2008, Ogorodnikova et al., 2008) as well as in marine algae (Gerwick, 1994).

Divinyl ethers are involved in plant defense. In tobacco and potato accumulated CA and CnA was detected after treatment with pathogens (Weber et al., 1999, Göbel et al., 2002). However, only in defined host-pathogen-interactions the function of divinyl ethers in plant defense was demonstrated (Fammartino et al., 2007).

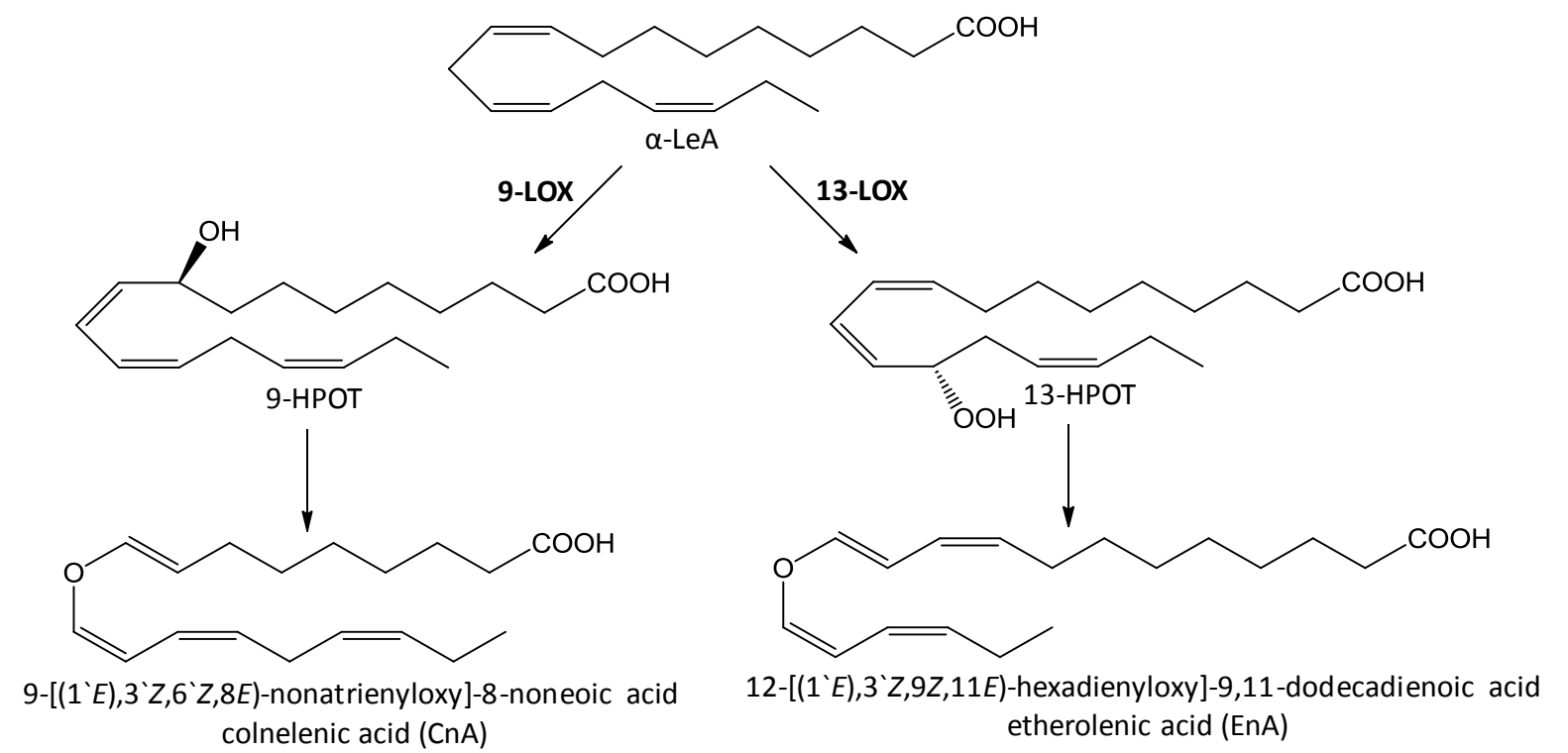

Figure 1.6: DES pathway for the conversion of $\alpha$-LeA (adapted from (Hamberg, 2004)). 9- and 13-LOX catalyze the introduction of molecular oxygen into fatty acid hydroperoxides to form divinyl ethers. CnA derives from 9HPOT and EnA from 13-HPOT. Abbreviations: 9-LOX: 9-lipoxygenase, 13-LOX: 13-lipoxygenase, 9-HPOT: 9hydroperoxy octadecatrienoic acid, 13-HPOT: 13-hydroperoxy octadecatrienoic acid, CnA: colnelenic acid, EnA: etherolenic acid.

\subsubsection{Epoxy alcohol synthase}

The epoxy alcohol synthase (EAS) is the fourth member of the CYP74-enzymes. The first CDNA of EAS was found in a marine invertebrate, the lancelet Branchiostoma floridae, and identified as CYP74enzyme (Andreou et al., 2009, Lee et al., 2008). In plants only trihydroxy products that might arise from the EAS pathway, have been found in Solanaceae like potatoes and in rice upon pathogen infection (Feussner and Wasternack, 2002). 
The synthesis of epoxyhydroxy fatty acids by intramolecular rearrangement of molecular oxygen of the hydroperoxy fatty acid is catalyzed by EAS. The conversion of 9-and 13-hydroperoxides derived from $\alpha$-LeA by EAS is shown in Figure 1.7. The resulting oxylipins seem to be involved in pathogen defense reactions (Blée, 1998b, Feussner and Wasternack, 2002).

As shown in Figure 1.2 epoxy alcohols can also be generated by PXG. It should be emphasized at this point, that this reaction is entirely unrelated to CYP74-catalysis and the stereochemistry of this substances is different. PXG catalyzes inter- and intramolecular transfer of oxygen from the hydroperoxides to generate epoxy alcohols as well as fatty acid epoxides, respectively (Blée, 1998a, Hanano et al., 2006).

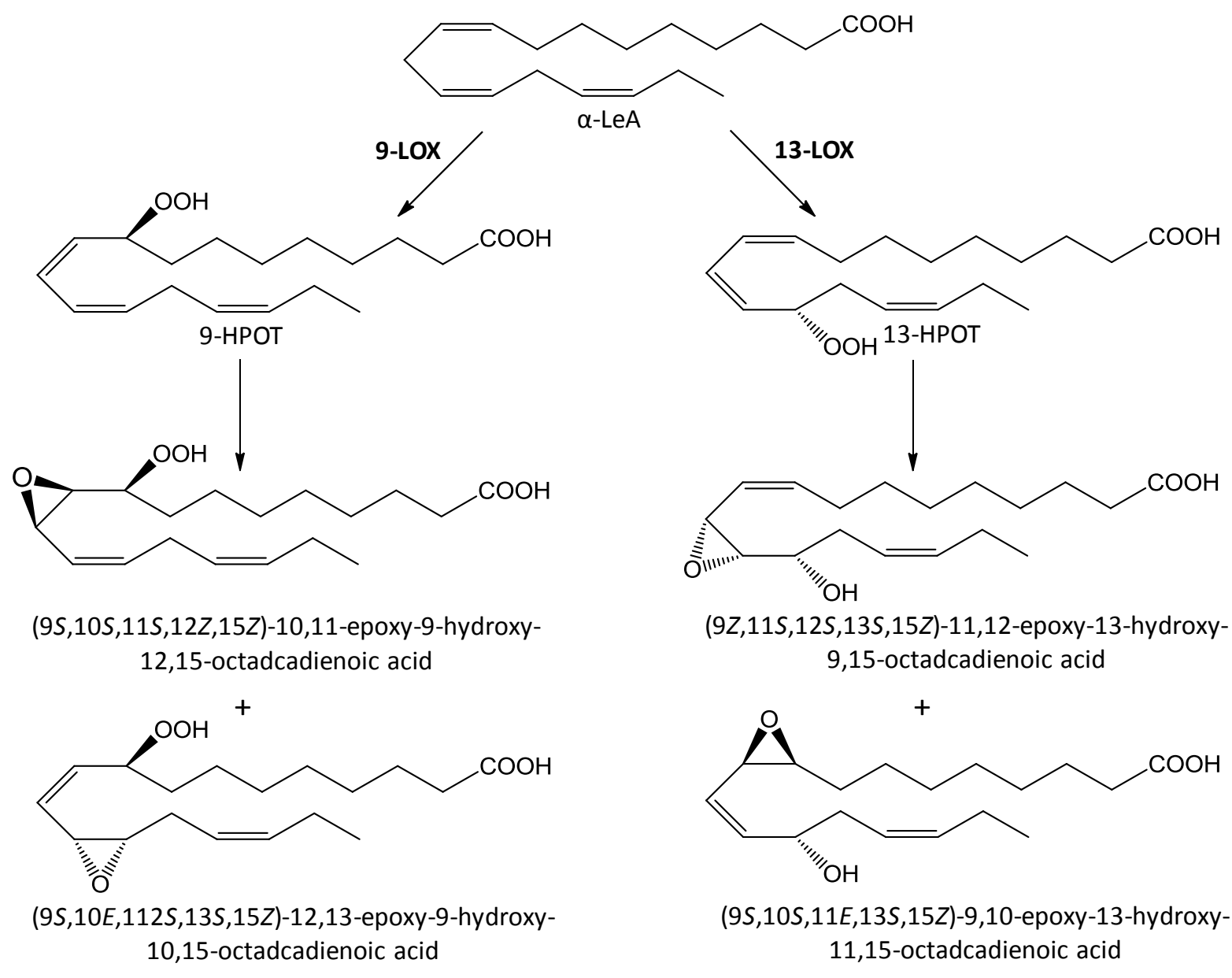

Figure 1.7: EAS pathway for the conversion of $\alpha$-LeA. Epoxyhydroxy fatty acids were generated by intramolecular rearrangement of molecular oxygen of the hydroperoxy fatty acids. Abbreviations: 9-LOX: 9lipoxygenase, 13-LOX: 13-lipoxygenase, 9-HPOT: 9-hydroperoxy octadecatrienoic acid, 13-HPOT: 13hydroperoxy octadecatrienoic acid 


\subsubsection{Proposed reaction mechanisms of CYP74-enzymes}

CYP74-enzymes have high similarities in their amino acid sequences as well as in their substrate specificities (1.3). Kuroda and coworkers showed that AOS, HPL and DES of the CYP74-family have common reaction mechanisms because of using the same substrates and formation of a common intermediate, an epoxy allylic carbocation, which arises from the fatty acid hydroperoxide (Kuroda et al., 2005). After solving the structures of AOS from Arabidopsis (AtAOS) and guayule (PaAOS) as well as after site-directed mutagenesis analysis reaction mechanisms for CYP74-enzyme catalysis were proposed. Several conserved amino acids were identified to play important roles in catalysis (Lee et al., 2008, Li et al., 2008, Toporkova et al., 2008, Schaller and Stintzi, 2009,Brash, 2009).

In Figure 1.8the proposed reaction mechanism for the CYP74-enzymes AOS and HPL is shown(Schaller and Stintzi, 2009). In the active site of the enzyme the heme iron is bound to the terminal oxygen of the hydroperoxyl group at position $\mathrm{C}_{13}$ in the hydroperoxide substrate and the second oxygen of the hydroperoxide is bound to an asparagine residue (Asn321 in AtAOS (Figure 1.8)) of the AOS enzyme. Homolytic cleavage of the oxygen bond (O-O bond) leads to an alkoxyl radical $\left(\mathrm{RO}^{\circ}\right)$ and an iron-oxo complex (Fe(IV)-OH). The alkoxyl radical interacts with the double bond between $C_{11}$ and $C_{12}$ and forms an epoxide at $C_{12}$ and $C_{13}$ and a radical $\left(C^{\circ}\right)$ at $C_{11}$. This radical is the common intermediate in the proposed AOS and HPL reaction mechanisms. In AOS sequences is a highly conserved phenylalanine residue (Phe137 in AtAOS (Figure 1.8)). Oxidation of the $C_{11}$ radical by the iron-oxo complex yields carbocation $\left(\mathrm{C}^{+}\right)$at $\mathrm{C}_{11}$. The Phe residue may be essential for AOS activity by stabilizing the intermediately formed carbon-centred substrate radical. Proton abstraction leads to formation of allene oxide. In HPL sequences is at this particular position instead of the Phe a leucine residue (Leu). This Leu is unable to stabilize the reactive product intermediate and thus leads to the formation of an unstable hemiacetal. The hemiacetal spontaneously dissociates into short chain aldehydes (Lee et al., 2008, Li et al., 2008, Toporkova et al., 2008, Schaller and Stintzi, 2009,Brash, 2009).

This concept was analyzed by Lee and coworkers by site-directed mutagenesis in AtAOS. Substitution of Phe137 into Leu137 led to alteration of enzyme catalysis (Lee et al., 2008).

Overall the epoxy allylic radical is the branching point in catalysis. As proposed by Brash (Brash, 2009) this radical is the intermediate for the synthesis of allene oxides (AOS), hemiacetals and aldehydes (HPL), divinyl ethers (DES) as well as for epoxy alcohols (EAS). 


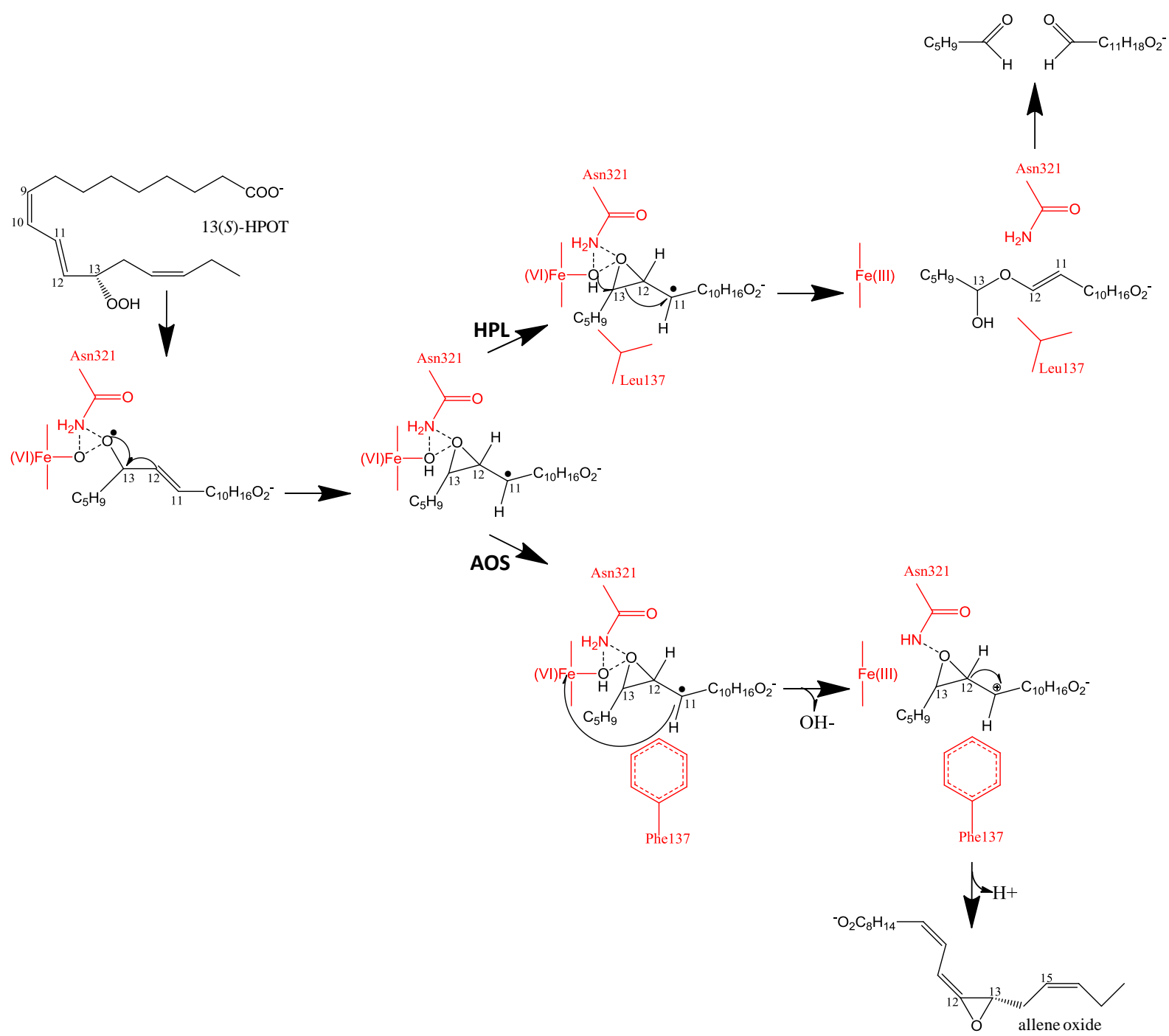

Figure 1.8: Proposed reaction mechanisms for CYP74-enzymes (adapted from (Schaller and Stintzi, 2009)). Oxygen of hydroperoxide substrate binds to the heme iron and to Asn321 of the enzyme. Homolytic cleavage leads to the formation of an epoxy allylic radical as intermediate for both AOS- and HPL-catalysis. AOS catalyzes the oxidation by the iron-oxo complex and the formation of a carbocation, which is encouraged by Phe137. Proton abstraction leads to an allene oxide. HPL catalyzes the formation of a hemiacetal, which decomposes to short chain aldehydes. Amino acid residues are numbered according to the solved structure of Arabidopsis AOS (Lee et al., 2008). Abbreviations: 13(S)-HPOT: 13-hydroperoxy octadecatrienoic acid, Asn321: asparagine 321, Phe137: phenylalanine 137, Leu137: leucine137, AOS: allene oxide synthase, HPL: hydroperoxide lyase.

From the epoxy allylic radical further reaction can be by radical (blue) or ionic (red) routes (Figure 1.9) (Brash, 2009). Catalysis of allene oxides and divinyl ethers occurs through ionic pathway where the radical intermediate is first converted into an epoxy allylic carbocation. Proton loss leads to the formation of allene oxides by AOS. Cleavage of the epoxy carbon double bond leads to the formation of a vinyl ether cation which can be converted to divinyl ethers by DES through proton loss.

Brash proposed that HPL products can be synthesized through the radical as well as through the ionic route (Figure 1.9) (Brash, 2009). In the ionic route $\mathrm{OH}^{-}$attacks the vinyl ether cation and yield the hemiacetal which spontaneously dissociates to short chain aldehydes. Whereas in the radical route the carbon double bond of the epoxy allylic radical is cleaved and led to the formation of the vinyl ether radical. Than oxygen rebound yield the short-lived hemiacetal which dissociates to short chain 
aldehydes. Synthesis of epoxy alcohols can also be through the radical as well as through the ionic route.

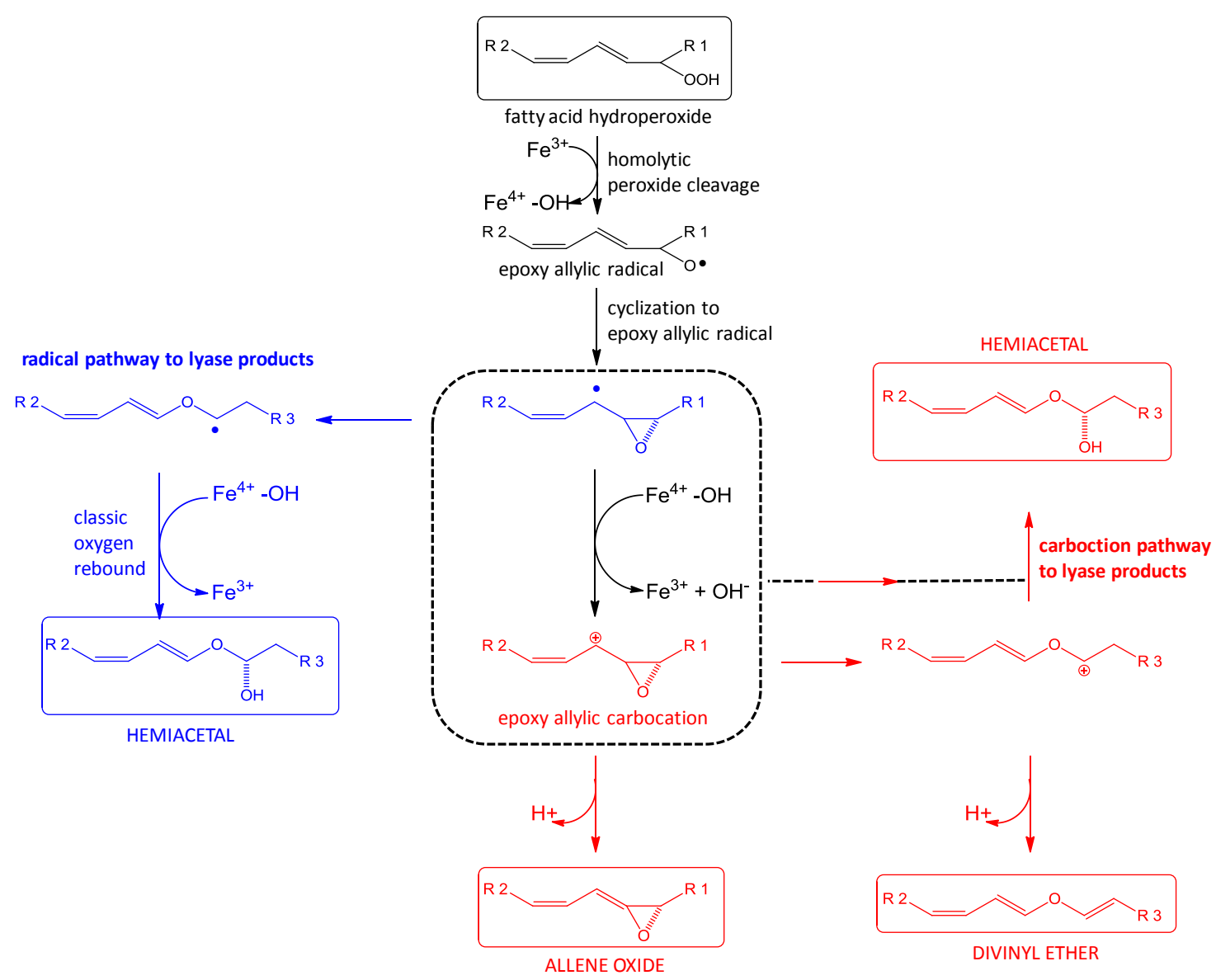

Figure 1.9: Proposed potential intermediates in radical and ionic pathways to CYP74 products (adapted from (Brash, 2009)). Homolytic cleavage of the fatty acid hydroperoxide leads to the formation of an alkoxyl radical and than cyclization to epoxy allylic radical occurs. This radical produces via the radical route the short-lived hemiacetal which spontaneously decomposes to short chain aldehydes (blue). The epoxyallylic radical can be converted to an epoxy allylic cation (black box). The carbocation can be metabolized via the ionic route to allene oxides and divinyl ethers. Hemiacetal and short chain aldehydes can also generated by the ionic route (red). Epoxy alcohols can also be synthesized via either the radical or ionic route (not shown).

Thus Brash proposed that synthesis of products by CYP74-enzymes can occur through either radical or ionic pathways which lead to potential radical or ionic intermediates (Brash, 2009). 


\subsection{Aim}

Aim of this study was to contribute to the characterization of enzymes of the CYP74-family. With the purified enzymes biochemical studies should be performed. By UV/vis and CD spectroscopy the heme-environment should be analyzed. Additionally the biochemical parameters of the enzyme should be determined. The substrate specificity against different hydroperoxides derived from LA, $\alpha$ LeA, $\gamma$-LeA and ARA and the optimal $\mathrm{pH}$ should be determined. In addition the products of the enzymatic reactions should be identified by radio-RP-HPLC and RP-HPLC/MS ${ }^{2}$.

A further aim of the study is the analysis and comparison of the reaction mechanism of the CYP74enzymes. Therefore conserved amino acids should be identified and their role in the reaction mechanism should be analyzed by site-directed mutagenesis.

It has been established that the catalytic trajectories of the different CYP74-enzymes are closely related (Brash, 2009) and that AOS and HPL seem to use a closely related mechanism and produce similar reaction intermediates in the different reactions. It was shown by single point mutagenesis that it is possible to convert the activity of the 13-hydroperoxide specific AOS from Arabidopsis into HPLactivity (Lee et al., 2008, Toporkova et al., 2008). In this study site-directed mutagenesis should be carried out in order to analyze if it is analogously possible to convert a possible unspecific AOS into HPL, DES or EAS. On the other hand, it will be investigated if a HPL can be converted into AOS, DES or EAS.

A fourth CYP74-enzyme EAS was discovered in marine invertebrates (Lee et al., 2008). For comparison with the other three CYP74s the EAS from Branchiostoma floridae should be characterized. Therefore the biochemical parameters as well as the reaction products were analyzed. In addition site-directed mutagenesis should be carried out in order to investigate if the concept established for AOS- and HPLactivity can also be applied on EAS. 


\section{Material and Methods}

The molecular and microbiological experiments were done as described in Current Protocols in Molecular Biology (Ausubel et al., 1993). The glassware was heat sterilized ( $8 \mathrm{~h}$ at $180{ }^{\circ} \mathrm{C}$ ) and sterile plastic articles were used. Furthermore double distilled water $\left(\mathrm{ddH}_{2} \mathrm{O}\right)$ was used.

\subsection{Materials}

\subsubsection{Chemicals}

All chemicals used in this study were purchased from Sigma-Aldrich (Steinheim, Germany) or from Carl Roth \& Co. (Karlsruhe, Germany) unless stated otherwise. Agarose was obtained from Biozym Scientific $\mathrm{GmbH}$ (Hess. Oldendorf, Germany), while the fatty acids were acquired from Cayman Chemicals (Ann Harbor, MI, USA) as well as from Enzo Life Sciences GmbH (Lörrach, Germany) and the restriction enzymes from MBI Fermentas (St. Leon-Rot, Germany).

\subsubsection{Molecular biology reagents}

Molecular biology kits

Nucleo Spin ${ }^{\oplus}$ Plasmid Kit

Big Dye ${ }^{\circledR}$ Terminator v3.1 Cycle Sequencing Kit

Macherey-Nagel (Düren, Germany)

Applied Biosystems (Darmstadt, Germany)

\section{DNA-Ladder}

Gene Ruler ${ }^{\text {TM }} 1 \mathrm{~kb}$ DNA-Ladder

MBI Fermentas (St. Leon-Rot, Germany)

\section{Protein Marker}

Protein Molecular Weight Marker

MBI Fermentas (St. Leon-Rot, Germany)

\section{Enzymes}

Phusion ${ }^{\mathrm{TM}}$ DNA Polymerase

Finnzyme New England Biolabs $\mathrm{GmbH}$ (Frankfurt am Main, Germany)

Pfu Polymerase ${ }^{\mathrm{TM}}$

MBI Fermentas (St. Leon-Rot, Germany)

Tfl DNA Polymerase ${ }^{\mathrm{TM}}$

Biozym Scientific GmbH(Hess. Oldendorf, Germany) 


\section{DNA}

The cDNA of PpAOS1, PpHPL, StDES and AsDES was kindly provided by Dr. Michael Stumpe (GeorgAugust-University, Göttingen, Germany), and the cDNA of PpAOS2 was kindly provided by Dr. Ellen Hornung (Georg-August-University, Göttingen, Germany).

The synthetic cDNA of BfEAS was ordered from GENEWIZ (South Plainfield, NJ, USA).

\subsubsection{Media}

Bacterial media(Ausubel et al., 1993)

Luria Bertani (LB)-medium:

$\begin{array}{ll}\text { Peptone } & 10 \mathrm{~g} / \mathrm{L} ; 0.1 \%(\mathrm{w} / \mathrm{v}) \\ \text { Yeast extract } & 5 \mathrm{~g} / \mathrm{L} ; 0.05 \%(\mathrm{w} / \mathrm{v}) \\ \mathrm{NaCl} & 10 \mathrm{~g} / \mathrm{L} ; 0.1 \%(\mathrm{w} / \mathrm{v})\end{array}$

in $\mathrm{ddH}_{2} \mathrm{O}$ and autoclaved for $20 \mathrm{~min}$ at $120^{\circ} \mathrm{C}$

2x YT medium:

$$
\begin{array}{ll}
\text { Peptone } & 30 \mathrm{~g} / \mathrm{L} ; 0.3 \%(\mathrm{w} / \mathrm{v}) \\
\text { Yeast extract } & 20 \mathrm{~g} / \mathrm{L} ; 0.2 \%(\mathrm{w} / \mathrm{v}) \\
\mathrm{NaCl} & 5 \mathrm{~g} / \mathrm{L} ; 0.05 \%(\mathrm{w} / \mathrm{v}) \\
\text { in } \mathrm{ddH}_{2} \mathrm{O} \text { and autoclaved for } 20 \mathrm{~min} \text { at } 120{ }^{\circ} \mathrm{C}
\end{array}
$$

LB agar plates: $\quad 1.5 \%(\mathrm{w} / \mathrm{v})$ agar was added to the LB-medium

Antibiotics: $\quad$ For selection of plasmid containing colony antibiotics were added to the medium:

\begin{tabular}{|c|c|c|c|c|}
\hline organism & strain & genotype & reference & $\begin{array}{l}\text { purchased } \\
\text { from }\end{array}$ \\
\hline $\begin{array}{l}\text { Escherichia coli } \\
\text { (E. coli) }\end{array}$ & XL1-Blue & $\begin{array}{l}\text { recA1endA1gyrA96 thi- } \\
\text { 1hsdR17supE44 } \\
\text { relA1lac[F'proAB lac9zM15 } \\
\text { Tn19 (Tetr)] }\end{array}$ & $\begin{array}{l}\text { (Bullock et al., } \\
\text { 1987) }\end{array}$ & $\begin{array}{l}\text { Stratagene } \\
\text { (Berlin, } \\
\text { Germany) }\end{array}$ \\
\hline E. coli & BL21 Star & $\begin{array}{l}\text { FompT hsdS } S_{B}\left(r_{B} m_{B}^{-}\right) \text {gal } \\
\text { dcm ara B::T7 RNAP-tetA }\end{array}$ & & $\begin{array}{l}\text { Invitrogen } \\
\text { (Karlsruhe, } \\
\text { Germany) }\end{array}$ \\
\hline E. coli & SG13009[pREP4] & 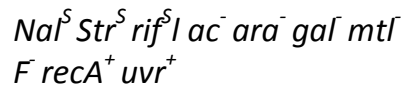 & $\begin{array}{l}\text { (Gottesman } \\
\text { et al., 1981) }\end{array}$ & $\begin{array}{l}\text { Qiagen (Hilden, } \\
\text { Germany) }\end{array}$ \\
\hline
\end{tabular}

$$
\begin{array}{lll}
\text { - } & \text { Carbenicillin: } & 100 \mu \mathrm{gg} / \mathrm{ml} \\
\text { - } & \text { Kanamycin: } & 25 \mu \mathrm{g} / \mathrm{ml}
\end{array}
$$

\section{Bacterial strains}

Table 2.1: Bacterial strains used. 


\subsubsection{Vectors}

Table 2.2: Vectors used.

\begin{tabular}{ccc}
\hline vector & selection marker & source \\
\hline pET24b & Kanamycin & Novagen/Merck Chemicals (Darmstadt, Germany) \\
pET28a & Kanamycin & Novagen/Merck Chemicals (Darmstadt, Germany) \\
pQE30 & Carbenicillin, Kanamycin & Qiagen (Hilden, Germany) \\
\hline
\end{tabular}

\subsection{Technical equipment}

Sterile bench Prett| ${ }^{\circledast}$ Telstar Bio II A

Thermocycler

Mastercycler personal

Mastercycler gradient

Centrifuge 5415D

Centrifuge 5417R

Centrifuge 5810R

Avanti $^{\mathrm{TM}}$ J-25 Centrifuge

Mini-PROTEAN Tetra System III

Raytest IDA gel documentation system
Telstar (Terrassa, Spain)

Eppendorf AG (Hamburg, Germany)

Eppendorf AG (Hamburg, Germany)

Eppendorf AG (Hamburg, Germany)

Eppendorf AG (Hamburg, Germany)

Eppendorf AG (Hamburg, Germany)

Beckman Coulter (Krefeld, Germany)

Bio-Rad Laboratories (Hercules, A, USA)

Herolab (Wiesloch, Germany)

Sonifier ${ }^{\circledR}$ Cell Disruptor B15Branson(Dietzenbach-Steinberg, Germany)

Corning ${ }^{\circledR}$ Spin- $X^{\circledR}$ UF Concentrators

Life Sciences (Lowell, MA, USA)

ÄKTAprime ${ }^{\mathrm{TM}}$ plus

GE Healthcare (Munich, Germany)

Ultrospec 1100 pro Photometer

GE Healthcare (Munich, Germany)

CARY 100 Bio UV-vis Spectrophotometer

Varian(Darmstadt, Germany)

Chirascan $^{\text {TM }}$ CD SpectrometerAppliedPhotophysicsLtd.(Leatherhead,

United Kingdom)

1100 series HPLC systemAgilent Technologies (Waldbronn, Germany)

LCQ Ion trap mass spectrometer

Thermo Finnigan (San Jose, CA, USA)

Surveyor HPLC system

Thermo Finnigan (San Jose, CA, USA)

Radio detector

Raytest (Straubenhardt, Germany) 


\subsection{Methods}

\subsubsection{Amplification of specific DNA fragments by polymerase chainreaction (PCR)}

Polymerase chain reaction (PCR) (Mullis et al., 1986) allows the exponential amplification of a specific DNA fragment. The PCR reaction mixture contains the template DNA, a heat stable polymerase, the four desoxyribonucleotides adenine, cytosine, guanine, and thymine, and two sequence specific primers. The PCR is performed in several steps with different temperatures. In the first step (denaturation) the double stranded DNA is separated into single strands $\left(92-98^{\circ} \mathrm{C}\right)$. In the second step (annealing) the sequence specific primers bind to the single stranded DNA $\left(55-60^{\circ} \mathrm{C}\right.$ ), and in the third step (elongation) DNA strands complementary to the template DNA are synthesized $\left(68-74{ }^{\circ} \mathrm{C}\right)$.

\subsubsection{Site-directed mutagenesis}

Site-directed mutagenesis in the amino acid sequence of PpHPL, PpAOS1, StDES, AsDES and BfEAS

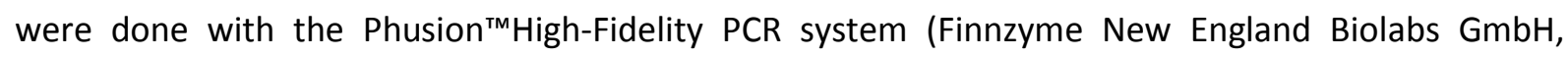
Frankfurt am Main, Germany) or $\mathrm{Pfu}^{\mathrm{TM}}$ DNA polymerase. For this oligonucleotide primers which contained the desired mutation and were complementary to each other were used. Primers contain additionally desired recognition sites for analysis by DNA restriction with restriction endonucleases. Using these primers and the Phusion ${ }^{\mathrm{TM}}$ DNA polymerase or $\mathrm{Pfu}^{\mathrm{TM}}$ DNA polymerase the plasmid with the desired point mutation was amplified. Subsequently the template DNA was digested with Dpnl.

Reaction mixture for Phusion ${ }^{\mathrm{TM}}$ High-Fidelity PCR system (Vol $\left.=50 \mu \mathrm{l}\right)$ :

\begin{tabular}{|c|c|}
\hline $10 \mu \mathrm{l}$ & $5 x$ Phusion $^{\text {TM }} H F$ reaction buffer \\
\hline $1 \mu \mathrm{l}$ & 10 mM dNTP-Mix \\
\hline $2 \mu \mathrm{l}$ & $10 \mu \mathrm{M}$ 3' Primer \\
\hline $2 \mu \mathrm{l}$ & $10 \mu \mathrm{M}$ 5' Primer \\
\hline $0.5 \mu \mathrm{l}$ & Phusion $^{\mathrm{TM}}$ DNA polymerase \\
\hline $1 \mu \mathrm{l}$ & 50 ng DNA \\
\hline
\end{tabular}

filled up with $\mathrm{ddH}_{2} \mathrm{O}$ to $50 \mu \mathrm{l}$

PCR program:

1. Denaturation: $2 \min$ at $98^{\circ} \mathrm{C}$

2. Denaturation: $\quad 30 \mathrm{~s}$ at $98^{\circ} \mathrm{C}$

3. Annealing: $\quad 30 \mathrm{~s}$ at $55^{\circ} \mathrm{C}$

4. Elongation: $\quad 30 \mathrm{~s} / \mathrm{kb}$ at $72{ }^{\circ} \mathrm{C}$ 
Repeat step 2 through 4: 16 times for single amino acid changes and 18 times for multiple amino acid deletions

Reaction mixture for $\mathrm{Pfu}^{\mathrm{TM}}$ DNA polymerase $(\mathrm{Vol}=50 \mu \mathrm{l})$ :

$\begin{array}{ll}5 \mu \mathrm{l} & 10 x \mathrm{Pfu}^{\mathrm{TM}} \text { reaction buffer with } \mathrm{MgCl}_{2} \\ 1 \mu \mathrm{l} & 10 \mathrm{mM} \text { dNTP-Mix } \\ 2 \mu \mathrm{l} & 10 \mu \mathrm{M} \text { 3' Primer } \\ 2 \mu \mathrm{l} & 10 \mu \mathrm{M} \text { 5' Primer } \\ 1 \mu \mathrm{l} & \mathrm{Pfu}^{\mathrm{TM}} \text { DNA polymerase } \\ 1 \mu \mathrm{l} & 50 \mathrm{ng} \text { DNA } \\ \text { filled up with } \mathrm{ddH}_{2} \mathrm{O} \text { to } 50 \mu \mathrm{l}\end{array}$

PCR program:

1. Denaturation:

2 min at $95^{\circ} \mathrm{C}$

2. Denaturation: $30 \mathrm{~s}$ at $95^{\circ} \mathrm{C}$

3. Annealing: $30 \mathrm{~s}$ at $55^{\circ} \mathrm{C}$

4. Elongation: $2 \mathrm{~min} / \mathrm{kb}$ at $72{ }^{\circ} \mathrm{C}$

Repeat step 2 through 4: 16 times for single amino acid changes and 18 times for multiple amino acid deletions

Restriction of the maternal DNA:

For the digestion of the template DNA $1 \mu \mathrm{l} \mathrm{Dpnl} \mathrm{was} \mathrm{added} \mathrm{and} \mathrm{incubated} \mathrm{for} 1 \mathrm{~h}$ at $37^{\circ} \mathrm{C}$.

\subsubsection{Colony PCR}

Colony PCR was applied for the identification of positive transformants. Therefore each bacterial colony was picked with a toothpick, transferred into a PCR tube containing $10 \mu \mathrm{H}_{2} \mathrm{O}$, and used as template for the PCR. Furthermore the colonies were streaked in parallel on a selection plate and incubated over night at $37^{\circ} \mathrm{C}$.

$\begin{array}{lll}\text { Reaction mixture }(\mathrm{Vol}=25 \mu \mathrm{l}): & 1.25 \mu \mathrm{l} & \begin{array}{l}\text { 20xTfl DNA polymerase } \\ \text { bufm }\end{array} \\ & 0.5 \mu \mathrm{l} & 10 \mathrm{mM} \text { dNTP-Mix } \\ 1.5 \mu \mathrm{l} & 10 \mu \mathrm{M} 3^{\prime} \text { Primer } \\ 1.5 \mu \mathrm{l} & 10 \mu \mathrm{M} 5^{\prime} \text { Primer } \\ 0.25 \mu \mathrm{l} & \text { Tfl polymerase } \\ 2.5 \mu \mathrm{lm} & 25 \mathrm{mM} \mathrm{MgCl}_{2}\end{array}$


$10 \mu \mathrm{l}$

$\mathrm{ddH}_{2} \mathrm{O}$ with bacterial colony as template

filled up with $\mathrm{ddH}_{2} \mathrm{O}$ to $25 \mu \mathrm{l}$

PCR Program:

1. Denaturation:

2. Denaturation:

3. Annealing:

4. Elongation:
2 min at $94^{\circ} \mathrm{C}$

30 s at $94^{\circ} \mathrm{C}$

$30 \mathrm{~s}$ at $55^{\circ} \mathrm{C}$

$1 \mathrm{~min} / \mathrm{kb}$ at $72^{\circ} \mathrm{C}$

Repeat step 2 through 4: 10 times

5. Denaturation:

30 s at $94^{\circ} \mathrm{C}$

6. Annealing:

$30 \mathrm{~s}$ at $55^{\circ} \mathrm{C}$

7. Elongation:

$1 \mathrm{~min} / \mathrm{kb}$ at $72{ }^{\circ} \mathrm{C}+5 \mathrm{~s}$ time increment

Repeat step 5 through 7: 15 times
8. Elongation:
5 min at $72^{\circ} \mathrm{C}$

\subsubsection{DNA precipitation}

For precipitation of amplified DNA fragments (2.3.1) 1/10 volume of $3 \mathrm{M} \mathrm{NaAc}$ and 2.5 time the volume of $98 \% \mathrm{EtOH}$ was added to the reaction mix, gently mixed, and incubated for $30 \mathrm{~min}$ at$20{ }^{\circ} \mathrm{C}$. Then the mixture was centrifuged for $15 \mathrm{~min}$ at $20.000 \times \mathrm{g}$ at $4{ }^{\circ} \mathrm{C}$. After centrifugation the supernatant was removed and the precipitate was washed with $25 \mu \mathrm{l} 70 \% \mathrm{EtOH}$ and centrifuged again for $5 \mathrm{~min}$ at $20.000 \times \mathrm{g}$ at $4{ }^{\circ} \mathrm{C}$. Finally the supernatant was removed again, the precipitated DNA was dried for $5-10 \mathrm{~min}$ at $65^{\circ} \mathrm{C}$, dissolved in $10 \mu \mathrm{lddH_{2 }} \mathrm{O}$ and stored at $-20^{\circ} \mathrm{C}$.

\subsubsection{DNA restriction}

To verify the presence of the correct mutation in the plasmid the DNA was analyzed by restriction analysis. Therefore the double stranded DNA was incubated with restriction endonucleases overnight at $37{ }^{\circ} \mathrm{C}$. Restriction endonucelases cleave double stranded DNA sequence specifically by hydrolysis of covalent bonds.

$\begin{array}{lll}\text { Restriction mixture }(\mathrm{Vol}=20 \mu \mathrm{l}): & 15 \mu \mathrm{l} & \text { of the colony PCR mixture } \\ 2 \mu \mathrm{l} & 10 x \text { reaction buffer } \\ 1 \mu \mathrm{l} & \text { Restriction enzyme } \\ 2 \mu \mathrm{l} & \mathrm{H}_{2} \mathrm{O}\end{array}$




\subsubsection{Sequencing}

To determine the correct nucleotide sequences of the plasmids sequencing analysis according to Sanger (Sanger et al., 1977) were done. Therefore the Big Dye ${ }^{\circledR}$ Terminator v3.1 Cycle Sequencing Kit (Applied Biosystems, Darmstadt, Germany) was used as described below. For DNA precipitation $1 \mu \mathrm{l}$ $125 \mathrm{mM}$ EDTA, $1 \mu \mathrm{l} 3 \mathrm{M} \mathrm{NaAc}$ and $50 \mu \mathrm{l} 98 \%$ EtOH was added, gently mixed and incubated for 5 min at $4{ }^{\circ} \mathrm{C}$. Afterwards the DNA was precipitated by centrifugation for $15 \mathrm{~min}$ at $20.000 \times \mathrm{g}$ at $4{ }^{\circ} \mathrm{C}$. The resulting pellet was washed with $70 \mu \mathrm{l} 70 \% \mathrm{EtOH}$ and centrifuged again for $5 \min 20.000 \mathrm{~g} \times$ at $4{ }^{\circ} \mathrm{C}$. Subsequently the supernatant was removed and the precipitate was dried for 1 min at $65^{\circ} \mathrm{C}$, taken up in $15 \mu \mathrm{l}$ formamide and stored at $-20^{\circ} \mathrm{C}$.

$\begin{array}{lll}\text { Reaction mixture }(\mathrm{Vol}=10 \mu \mathrm{l}): & 1 \mu \mathrm{l} & 200-400 \text { ng template DNA } \\ 1 \mu \mathrm{l} & 8 \text { pmol Primer } \\ 1.5 \mu \mathrm{l} & \text { Sequencing mix } \\ 1.5 \mu \mathrm{l} & \text { Sequencing reaction buffer }\end{array}$

filled up with $\mathrm{ddH}_{2} \mathrm{O}$ to $10 \mu \mathrm{l}$

Temperature profile of sequencing reaction:
1. Denaturation:
1 min at $96^{\circ} \mathrm{C}$
2. Denaturation:
$10 \mathrm{~s}$ at $96^{\circ} \mathrm{C}$
3. Annealing:
$15 \mathrm{~s}$ at $55^{\circ} \mathrm{C}$
4. Elongation:
4 min at $60^{\circ} \mathrm{C}$

Repeat step 2 through 4: $\quad 25$ times

\subsubsection{Agarose gel electrophoresis}

\subsubsection{Separation of DNA fragments by agarose gel electrophoresis}

The separation and size determination of DNA fragments was performed by agarose gel electrophoresis. For this $1 \%(w / v)$ agarose gel was used and prepared with 1 X TAE buffer. Before application $1 / 10$ of the volume DNA loading buffer was added to the samples. As DNA standard GeneRuler 1kb DNA-Ladder (MBI Fermentas, St. Leon-Rot, Germany) was used. The electrophoresis was performed for 20-30 min at $120 \mathrm{~V}$ in $1 \mathrm{X}$ TAE buffer. Afterwards the agarose gels were incubated in an ethidium bromide solution( $2 \mu \mathrm{g} / \mathrm{L})$ for 10-15 min and briefly destained in water. Ethidium bromide can intercalate between the bases of the DNA and emits fluorescence under UV light. The DNA bands were visualized under UV light and documented with the raytest IDA gel documentation system (Herolab, Wiesloch, Germany). 


$\begin{array}{lll}\text { TAE buffer (50x): } & 2 \mathrm{M} & \text { Tris/HCl, pH } 8.0 \\ 1 \mathrm{M} & \text { Acetic acid } \\ 0.05 \mathrm{M} & \text { EDTA } \\ & & \\ \text { DNA sample buffer: } & 100 \mathrm{mM} & \text { EDTA, pH } 8.0 \\ 0.1 \%(\mathrm{w} / \mathrm{v}) & \text { SDS } \\ & 40 \%(\mathrm{v} / \mathrm{v}) & \text { Glycerol } \\ 0.25 \%(\mathrm{w} / \mathrm{v}) & \text { Bromphenol } \\ & 0.25 \%(\mathrm{w} / \mathrm{v}) & \text { Xylene cyanol }\end{array}$

\subsubsection{Cultivation of $E$. coli}

The E. coli strains used in this work were cultivated on solid or in liquid medium. The precultures were cultivated in liquid LB medium shaking at $200 \mathrm{rpm}$ at $37^{\circ} \mathrm{C}$. The expression cultures were incubated in liquid $2 \mathrm{x}$ YT shaking at $200 \mathrm{rpm}$ at various temperatures $\left(11^{\circ} \mathrm{C}\right.$ for $1 \mathrm{~d}, 28^{\circ} \mathrm{C}$ for $1 \mathrm{~d}$ or $16{ }^{\circ} \mathrm{C}$ for $3 \mathrm{~d}$ ). To select plasmid containing bacterial cells antibiotics were added. For selection of pET24b and pET28a clones kanamycin was added to the medium. The PQE30 clones were selected by adding both carbenicillin and kanamycin.

\subsubsection{Transformation of E. coli}

\subsubsection{Transformation}

For the transformation of plasmids frozen chemically competent $E$. coli cells were thawed on ice. Then $100 \mu \mathrm{l}$ of the cell suspension were added to $1 \mu \mathrm{l}$ of plasmid DNA or $10 \mu \mathrm{l}$ of precipitated mutated DNA (2.3.1.3) and incubated for at least $20 \mathrm{~min}$ on ice. Subsequently the cells were shocked for $45 \mathrm{~s}$ at $42{ }^{\circ} \mathrm{C}$ in a water bath. After incubation for 5 min on ice $900 \mu \mathrm{L}$ LB medium was added and then the mixture was incubated for $1.5 \mathrm{~h}$ shaking at $200 \mathrm{rpm}$ at $37^{\circ} \mathrm{C}$. Afterwards the cells were spun down for $1 \mathrm{~min}$ at $800 \mathrm{xg}$ and the supernatant was discarded. The cell precipitate was resuspended in the remaining medium and then spread on selective LB agar plates and incubated over night at $37^{\circ} \mathrm{C}$.

To analyze the transformed cells colony PCR was used (see 2.3.1.2). 


\subsubsection{Isolation of plasmid DNA fromE. coli}

For isolation of plasmid DNA the E. coli strain XL1-Blue was used. $5 \mathrm{ml}$ of LB medium were inoculated with cells from a selected bacterial colony and the appropriate antibiotics and were grown over night shaking at $200 \mathrm{rpm}$ at $37^{\circ} \mathrm{C}$. After harvesting the cells by centrifugation the isolation of the plasmid DNA was done according to the manual of the Nucleo Spin ${ }^{\circledR P l a s m i d ~ K i t ~(M a c h e r e y-N a g e l, ~ D u ̈ r e n, ~}$ Germany). This method is based on alkaline lysis according to Birnboim and Doly (Birnboim and Doly, 1979).

DNA was eluted from the column with $50 \mu \mathrm{lddH_{2 }} \mathrm{O}$ and then stored at $-20^{\circ} \mathrm{C}$.

\subsubsection{Heterologous expression of recombinant proteins in $E$. coli}

For expression of recombinant proteins the E. coli strain BL21 Star was used. First a preculture of the expression clones in LB medium with the appropriate antibiotics was prepared and grown over night shaking at $200 \mathrm{rpm}$ at $37^{\circ} \mathrm{C}$. The expression culture was inoculated with $1 / 20$ volume of the preculture consisting of $2 x$ YT medium which contained the appropriate antibiotics. The cells were incubated at $200 \mathrm{rpm}$ at $37{ }^{\circ} \mathrm{C}$ until they reached an $\mathrm{OD}_{600}$ of $0.6-0.8$. To ensure strong heme production $80 \mathrm{mg} / \mathrm{L} \delta$-amino levulinoic acid was added and $150 \mu \mathrm{M} 0.1 \mathrm{mM}$ ammonium ferric citrate. After an incubation of $15 \mathrm{~min}$ shaking at $200 \mathrm{rpm}$ at $30^{\circ} \mathrm{C}$ expression of recombinant protein was induced by the addition of IPTG to a final concentration of $0.1 \mathrm{mM}$. Then the cells were either incubated for $3 \mathrm{~d}$ shaking at $200 \mathrm{rpm}$ at $16^{\circ} \mathrm{C}$ or for $1 \mathrm{~d}$ at $11^{\circ} \mathrm{C}$ or for $1 \mathrm{~d}$ at $28^{\circ} \mathrm{C}$. Cells were harvested for $20 \mathrm{~min}$ at $3.000 \times \mathrm{g}$ at $4{ }^{\circ} \mathrm{C}$.

\subsubsection{Cell disruption of $E$. coli expression cultures (adapted from (Richardson et al., 1993))}

The harvested cells were lysed in accordance to (Richardson et al., 1993), with some modifications. Cells from $1 \mathrm{~L}$ culture were dissolved in $150 \mathrm{ml}$ Tris buffer. Lysozyme was added up to a final concentration of $0.2 \mathrm{mg} / \mathrm{ml}$ and the cells were incubated for $30 \mathrm{~min}$ while gently stirred at $4{ }^{\circ} \mathrm{C}$. Then the solution was centrifuged for $10 \mathrm{~min}$ at $3.000 \times \mathrm{g}$ at $4{ }^{\circ} \mathrm{C}$. The sedimented spheroblasts were dissolved in $50 \mathrm{ml}$ sodium phosphate buffer and frozen over night at $-80^{\circ} \mathrm{C}$.

While thawing the frozen cells protease inhibitor phenylmethylsulfonyl fluoride (PMSF) was added to a final concentration of $0.5 \mathrm{mM}$. Subsequently the cells were further lysed by using a sonifier cell disrupter (B15) from Branson (5x 1 min at $40 \%$ power and $40 \%$ pulse) on ice. Afterwards the cell debris was removed by centrifugation for $20 \mathrm{~min}$ at $50.000 \mathrm{xg}$. The resulting supernatant was used for further analysis. 
Tris buffer:

Sodium phosphate buffer:

$$
\begin{array}{ll}
100 \mathrm{mM} & \text { Tris/HCl, pH } 7.8 \\
20 \%(\mathrm{v} / \mathrm{v}) & \text { Glycerol }
\end{array}
$$

$\begin{array}{ll}10 \mathrm{mM} & \text { Sodium phosphate, } \mathrm{pH} 8.0 \\ 14 \mathrm{mM} & \text { MgAc } \\ 60 \mathrm{mM} & \text { KAc } \\ 0.1 \mathrm{mM} & \text { DTT }\end{array}$

\subsubsection{Purification of recombinant expressed proteins}

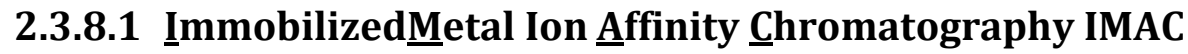

For the purification of recombinant proteins which have a polyhistidine sequence at the $\mathrm{N}$ - or $\mathrm{C}$ terminal end the immobilized metal ion affinity chromatography (IMAC) is used. In this chromatographic separation method the matrix of the column consists of immobilized metal ion groups, such as nickel, copper or cobalt. The polyhistidine residues of the recombinant proteins bind on the immobilized metal ions. Proteins can be eluted from the column by imidazole which replaces the polyhistidine residues at the metal ions.

For the IMAC a cell culture was harvested and lysed as described in 2.3.6 and 2.3.7. Afterwards the supernatant was applied to a $1 \mathrm{ml}^{-H_{i s T r a p}{ }^{\mathrm{TM}}} \mathrm{HP}$-column (GE Healthcare, Munich, Germany) which was connected to an ÄKTAprime ${ }^{\mathrm{TM}}$ plus system (GE Healthcare, Munich, Germany). The column was washed with $10-20 \mathrm{ml}$ of binding buffer. Then unspecific bound proteins were eluted with $10-20 \mathrm{ml}$ of $5 \%(\mathrm{v} / \mathrm{v})$ elution buffer. Finally protein was eluted from the column with a gradient from $5 \%$ $100 \%$ elution buffer within $20 \mathrm{~min}$ and a flow rate of $1 \mathrm{ml} / \mathrm{min}$. Meanwhile $2 \mathrm{ml}$ fractions were collected.

Binding buffer:

$\begin{array}{ll}50 \mathrm{mM} & \text { Sodium phosphate, } \mathrm{pH} 8.0 \\ 1 \mathrm{M} & \mathrm{NaCl} \\ 500 \mathrm{mM} & \text { Urea }\end{array}$

Elution buffer:

$\begin{array}{ll}50 \mathrm{mM} & \text { Sodium phosphate, } \mathrm{pH} 8.0 \\ 1 \mathrm{M} & \mathrm{NaCl} \\ 500 \mathrm{mM} & \text { Urea } \\ 300 \mathrm{mM} & \text { Imidazole }\end{array}$




\subsubsection{Sodium $\underline{\text { Dodecyl }} \underline{\text { Sulfate }} \underline{\text { Poly }} \underline{\text { Acrylamide }}$ Gel Electrophoresis (SDS-PAGE)}

To verify protein expression in E.coli and purity of recombinant protein SDS-PAGE according to Laemmli (Laemmli, 1970)was performed. In this method proteins are separated according to their molecular weight.

For this purpose $30 \mu \mathrm{l}$ of purified proteins were treated with $30 \mu \mathrm{l}$ of 2x SDS sample buffer and gently mixed. Afterwards the samples were denaturated for $5 \mathrm{~min}$ at $95^{\circ} \mathrm{C}$.

The Laemmli system consists of a separation gel, whose acrylamide concentration depends on the desired separation area and of a stacking gel, which overlays the polymerized separation gel. The separation gel was poured in unpolymerized condition between two glass plates and was immediately overlaid with ethanol. After polymerization of the separation gel the ethanol was removed with water from the separation gel surface. Subsequently the stacking gel was poured in and the sample comb was inserted. After polymerization the sample comb was removed carefully.

5-15 $\mu \mathrm{l}$ of the denaturated samples were loaded per well. Gels were run with $30 \mathrm{~mA}$ per gel for 1$1.5 \mathrm{~h}$. Afterwards the SDS-polyacrylamide gels were stained by Coomassie Brilliant Blue G250 and destained in destaining solution to decolorize the background of the gel.

Stacking gel (4.8\%):

Separating gel (10\%):

SDS-PAGE buffer:
$375 \mu l$

$240 \mu \mathrm{l}$

$3 \mu \mathrm{l}$

$6 \mu \mathrm{l}$

$885 \mu \mathrm{l}$

$1 \mathrm{ml}$

$1.33 \mathrm{ml}$

$3.1 \mu l$

$6.1 \mu \mathrm{l}$

$1.67 \mathrm{ml}$

$25 \mathrm{mM}$

$20 \mathrm{mM}$

$0.1 \%(w / v)$
$0.5 \mathrm{mM}$ Tris/HCl, $\mathrm{pH} 6.8 ; 0.4 \%$ (w/v) SDS $30 \%$ (w/v) Acrylamide/Bisacrylamide $25 \%(w / v)$ APS

TEMED

$\mathrm{ddH}_{2} \mathrm{O}$

1.5 M Tris/HCl, $\mathrm{pH} 8.8 ; 0.4 \%$ (w/v) SDS

$30 \%$ (w/v) Acrylamide/Bisacrylamide $25 \%(w / v)$ APS

TEMED

$\mathrm{ddH}_{2} \mathrm{O}$

Tris/ $\mathrm{HCl}, \mathrm{pH} 8.0$

Glycine

SDS 


$\begin{array}{lll}\text { SDS sample buffer (2x): } & 20 \mathrm{mM} & \text { Tris/HCl, pH } 6.5 \\ 4 \%(\mathrm{w} / \mathrm{v}) & \text { SDS } \\ 40 \%(\mathrm{v} / \mathrm{v}) & \text { Glycerol } \\ 10 \%(\mathrm{v} / \mathrm{v}) & \text { ß-Mercaptoethanol } \\ & 0.002 \%(\mathrm{w} / \mathrm{v}) & \text { Bromophenol blue } \\ \text { Coomassie staining solution: } & 0.002 \%(\mathrm{w} / \mathrm{v}) & \text { Coomassie brilliant Blue R250 } \\ & 40 \%(\mathrm{v} / \mathrm{v}) & \text { Methanol } \\ & 5 \%(\mathrm{v} / \mathrm{v}) & \text { Acetic acid } \\ & & \\ \text { Destaining solution: } & 30 \%(\mathrm{v} / \mathrm{v}) & \text { Ethanol } \\ & 5 \%(\mathrm{v} / \mathrm{v}) & \text { Acetic acid } \\ & 65 \%(\mathrm{v} / \mathrm{v}) & \text { ddH }{ }_{2} \mathrm{O}\end{array}$

\subsubsection{Substrate preparation and purification}

\subsubsection{Preparation of 9-hydroperoxy fatty acids}

For the preparation of 9-hydroperoxy fatty acids recombinant StLOX and AtLOX1 were used as described before (Stumpe et al., 2006a, Stumpe and Feussner, 2006). $2.5 \mathrm{mg}$ LA was added to a $10 \mathrm{ml}$ reaction mix and incubated in open tubes shaking for $30 \mathrm{~min}$ at RT.

For the protonation of the fatty acids $1 / 10$ of volume acetic acid was added. The isolation of the hydrophilic substances was done by extraction with diethyl ether. For the separation of the two phases the solution was centrifuged for $5 \mathrm{~min}$ at $3.220 \times \mathrm{g}$ at $4{ }^{\circ} \mathrm{C}$. The upper ether phase was removed and transferred into another tube and the extraction step was repeated. The diethyl ether was evaporized under streaming nitrogen. Subsequently the product was resolved in methanol, vortexed, and transferred into a new tube for storage at $-20^{\circ} \mathrm{C}$.

Incubation buffer:

$\begin{array}{ll}50 \mathrm{mM} & \text { Tris/HCl, pH } 7.5 \\ 500 \mathrm{mM} & \mathrm{NaCl} \\ 10 \% / \mathrm{v} / \mathrm{v}) & \text { Glycerol } \\ 0.1 \%(\mathrm{v} / \mathrm{v}) & \text { Tween } 20\end{array}$




\subsubsection{Preparation of 12-hydroperoxy fatty acids}

For the preparation of 12-hydroperoxy fatty acids cell pellets of recombinant PpLOX was resuspended in lysis buffer and subsequently digested as described in 2.3.10.1. Then $1 \mathrm{mg}$ ARA was added to the supernatant and incubated in open tubes shaking for $30 \mathrm{~min}$ at RT.

The extraction of the hydrophilic substances was performed as described in 2.3.10.1.

\subsubsection{Preparation of 13- and 17-hydroperoxy fatty acids}

For the preparation of 13- and 17-hydroperoxy fatty acids $100 \mathrm{mM}$ borate buffer, pH 10.4 was incubated with purified recombinant GmLOX1. $2.5 \mathrm{mg} \alpha$-LeA or $2.5 \mathrm{mg} \gamma$-LeA or $2.5 \mathrm{mg}$ LA were added for the generation of 13-hydroperoxides, whereas docosahexaenoic acid (DHA) was added for 17-hydroperoxides. The incubation and extraction was done as described in 2.3.10.1.

\subsubsection{Preparation of radio-labeled 9-and 13-hydroperoxy fatty acids}

For the preparation of radio-labeled hydroperoxy fatty acids, $\left[1-{ }^{14} \mathrm{C}\right]$-labeled substrates were used and prepared similar to the method described above in 2.3.10.1. To ensure high amounts of substrates, unlabeled fatty acids were additionally added.

\subsubsection{Purification of fatty acid}

It was necessary to purify DHA prior to using it as substrate due to autoperoxidation products. This was done by using a high performance liquid chromatography (HPLC). This method is for the separation and analysis of liquid mixtures of substances. The separation takes place on a column between a stationary and a mobile phase. The stationary phase is a fixed, inert, and highly porous column material like silica gel. The mobile phase is the substance which is dissolved in solvent and which should be analyzed. The mobile phase is pressed through the column under high pressure.

Via RP-HPLC (reversed phase) the peroxides can be separated from the fatty acid. By SP-HPLC (straight phase) the hydroxides and hydroperoxides as well as their positional isomers can be separated(Stumpe et al., 2005). 
HPLC Parameters:

RP-HPLC:

Column: EC 250/4 Nucleosil 120-5 C18 (250 x $4.6 \mathrm{~mm}, 5 \mu \mathrm{m}$ particle size; Macherey-Nagel, Düren, Germany)

Eluent: 90 : $10: 0.1$ (v:v:v) Methanol : Water : Acetic acid

Flow rate: $1 \mathrm{ml} / \mathrm{min}$

SP-HPLC:

Column:

$3 \mu \quad$ LUNA SILICA $(50 \times 4.6 \mathrm{~mm}, \quad 3 \mu \mathrm{m}$ particle size; Phenomenex, Aschaffenburg, Germany)

Eluent: $100: 1: 0.02$ (v:v:v) n-Hexane : Isopropanol : TFA

Flow rate: $1 \mathrm{ml} / \mathrm{min}$

\subsubsection{Analyzingenzyme activity}

\subsubsection{Photometric activity assay}

For the activity analysis it is usefully that the substrates of the CYP74-enzymes, the hydroperoxides produced by LOX, possess a conjugated double bound system which absorbs at 234-236 nm and therefore can be recorded by a spectrophotometer (Varian, Darmstadt, Germany) under UV light (Stumpe et al., 2006b).

For the analysis a preparation of reaction buffer, substrate, and enzyme was used. First a zero adjustment with buffer was done and then the substrate was added. The substrate concentration in the reaction mixture was determined. After that the reaction was started by adding the enzyme and the absorption curve at $234 \mathrm{~nm}$ was recorded.

The calculation of the substrate concentration was done by using the Lambert-Beer law. For this the concentration change $\Delta \mathrm{C} / \Delta \mathrm{t}$ has been determined through the measured extinction change $\Delta \mathrm{E} / \Delta \mathrm{t}$ :

$$
\frac{\Delta C}{\Delta t}=\frac{\Delta E}{\epsilon * \Delta t * d} * \frac{m M}{\min }
$$

The extinction coefficient for hydro(pero)xy fatty acids with a conjugated double bound system is $23 \mathrm{mM}^{-1} \mathrm{~cm}^{-1}\left(=23\left[\mu \mathrm{mol} / \mathrm{ml}^{-1} \mathrm{~cm}^{-1}\right)\right.$ at $234 \mathrm{~nm}$.

Reaction buffer: $\quad 50 \mathrm{mM}$ sodium phosphate 


\subsubsection{Determination of protein concentration with Bradford}

The determination of protein concentration was performed with the colorimetric method of Bradford (Bradford, 1976). This method is based on the fact that the color brilliant blue G250 shifts its absorption maximum from $465 \mathrm{~nm}$ to $595 \mathrm{~nm}$ in the presence of proteins.

For the measurement $1 \mathrm{ml}$ Bradford solution and $20 \mu \mathrm{l}$ protein were incubated for $5 \mathrm{~min}$ at RT. The absorption was measured with a spectrophotometer (Varian, Darmstadt, Germany) at $595 \mathrm{~nm}$. As blank $1 \mathrm{ml}$ Bradford solution with $20 \mu$ l buffer was used.

Bradford solution:

$\begin{array}{ll}425 \mathrm{ml} & \text { Water } \\ 50 \mathrm{ml} & \text { Phosphoric acid (85\%, v/v) } \\ 25 \mathrm{ml} & \text { EtOH }(96 \%, \mathrm{v} / \mathrm{v}) \\ 35 \mathrm{mg} & \text { Serva blue G } \\ \text { filled up with } \mathrm{ddH}_{2} \mathrm{O} \text { to } 1 \mathrm{~L}\end{array}$

\subsubsection{Analysis of products}

\subsubsection{RP-HPLC}

For the RP-HPLC analysis samples were evaporated and then dissolved in $80 \mu \mathrm{l} \mathrm{RP}$-solvent (Acetonitrile : Water : Acetic acid $50: 50: 0.1, \mathrm{v} / \mathrm{v} / \mathrm{v}$ ). The signals of the products were detected by a diode array detector (DAD).

Table 2.3: Flow diagram for the RP-HPLC separation.

\begin{tabular}{ccc}
\hline time $[\mathrm{min}]$ & \% eluent B & flow [ml/min] \\
\hline $\mathbf{0}$ & 0 & 0.2 \\
30 & 100 & 0.2 \\
32 & 100 & 0.2 \\
35 & 0 & 0.2 \\
\hline
\end{tabular}

\subsubsection{Radio-HPLC}

The radio-RP-HPLC uses additionally to the diode array detector a scintillation detector which detects the radioactive radiation. As substrates $\left[1-{ }^{14} \mathrm{C}\right]$-labeled hydroperoxides derived from LA and LeA was used (2.3.10.4).

The enzymatic conversions were typically performed in $1 \mathrm{ml} 100 \mathrm{mM}$ sodium phosphate buffer, pH 6.0 at RT under constant shaking for 30 min. After the incubation remaining substrate and 
products were extracted twice with $500 \mu \mathrm{l}$ diethyl ether. The diethyl ether phase was evaporated under nitrogen, the residue was resolved in $100 \mu \mathrm{l}$ methanol and evaporated again under nitrogen prior dissolving in $80 \mu \mathrm{l}$ Acetonitrile : Water : Acetic acid (50:50:0.1). Samples were then analyzed according to Blée (Blée and Joyard, 1996).

HPLC Parameters:

RP-HPLC:

Column:

LiChroCART ${ }^{\circledR}$ 125-4 LiChrospher ${ }^{\circledR} 100-R P-18 E C ~(5 \mu \mathrm{m}$ particle size; Merck, Darmstadt, Germany)

Eluent:

A: $50: 50: 0.1$ (v:v:v) Acetonitrile : Water : Acetic acid

B: $80: 20: 0.1(v: v: v)$ Acetonitrile : Water : Acetic acid

Flow rate: $1 \mathrm{ml} / \mathrm{min}$

Table 2.4: Flow diagram for the radio-RP-HPLC separation.

\begin{tabular}{ccc}
\hline time $[\mathrm{min}]$ & \% eluent B & flow [ml/min] \\
\hline 0 & 0 & 1 \\
25 & 0 & 1 \\
26 & 100 & 1 \\
36 & 100 & 1 \\
38 & 0 & 1 \\
\hline
\end{tabular}

\subsubsection{RP-HPLC/MS 2}

The coupling of HPLC with mass spectrometry (MS) is described as HPLC/MS ${ }^{2}$. The separation of the sample molecules occurs first by RP-HPLC to which the analysis by mass spectrometry is connected. The analytes are transferred and ionized into the vacuum of the mass spectrometer by electrospray ionization (ESI). The so formed ions are then analyzed with an ion trap according to their mass/charge ratios $(\mathrm{m} / \mathrm{z})$. Furthermore it is possible by using an ion trap mass spectrometer that the collision induced dissociation fragments the analytes into daughter ion fragments that can be analyzed by $\mathrm{MS}^{2}$.

The enzymatic conversions were performed as described in 2.3.13.2. As substrates unlabeled hydroperoxides derived from LA and LeA were used. For the HPLC/MS ${ }^{2}$ measurements the sample were dissolved in $50 \mu \mathrm{l}$ Acetonitrile: Water : Acetic acid $(50: 50: 0.1)$ and analyzed according to Brodhun (Brodhun et al., 2009). 
HPLC Parameters:

Device: Surveyor HPLC System (Thermo Finnigan, San Jose, CA, USA)

Column: EC $250 / 2$ Nucleosil $100-5$ C-18 $(250 \times 2.1 \mathrm{~mm}, 5 \mu \mathrm{m}$ particle size; Macherey-Nagel, Düren, Germany)

Running time: $45 \mathrm{~min}$

Eluent: A: 40 : 60 : 0.1 (v:v:v:) Acetonitrile : Water : Acetic acid B: $100: 0.1(\mathrm{v}: \mathrm{v})$ Acetonitrile : Acetic acid

Mass spectrometry parameters:

Device: LCQ ion trap mass spectrometer (Thermo Finnigan, San Jose, CA, USA)

Ionization: negative ESI

Source voltage/collision energy $4 \mathrm{kV} / 1 \mathrm{~V}$ Capillary tension and temperature: $27 \mathrm{~V}$ and $300^{\circ} \mathrm{C}$

Mass range: $m / 250-450$

Table 2.5: Flow diagram for the RP-HPLC/MS ${ }^{2}$ separation.

\begin{tabular}{cccc}
\hline time $[\mathrm{min}]$ & A \% & B \% & flow [ml/min] \\
\hline $\mathbf{0}$ & 80 & 20 & 0.2 \\
$\mathbf{1 0}$ & 80 & 20 & 0.2 \\
$\mathbf{3 0}$ & 0 & 100 & 0.2 \\
$\mathbf{3 5}$ & 0 & 100 & 0.3 \\
$\mathbf{4 0}$ & 0 & 100 & 0.3 \\
$\mathbf{4 4 . 5}$ & 80 & 20 & 0.3 \\
$\mathbf{4 5}$ & 80 & 20 & 0.2 \\
& 100 & 0 & 0.2 \\
\hline
\end{tabular}

\subsubsection{Biochemical characterization}

\subsubsection{Determination of pH optimum}

For the determination of the $\mathrm{pH}$ optimum of the investigated enzymes, the conversion of the substrate 13-HPOD to the corresponding products was measured by recording the decrease in absorbance at $234 \mathrm{~nm}$ with a CARY 100 Bio UV/vis spectrophotometer (Varian, Darmstadt, Germany). The reaction and analysis was carried out using various buffers with defined pH (see Table 2.6 and Table 2.7) with $30 \mu \mathrm{M}$ substrate solution and $0.1 \mathrm{nM}$ (PpAOS1) or $1 \mathrm{nM}$ (BfEAS) of enzyme. 
Table 2.6: Range $\mathrm{pH}$ and buffer used for measurements of PpAOS1.

\begin{tabular}{cc}
\hline buffer & pH range \\
\hline Acetate & $4.7-5.5$ \\
Phosphate & $5.5-8.0$ \\
Boric Acid-Borax & $8.0-10.5$ \\
\hline
\end{tabular}

Table 2.7: Range $\mathrm{pH}$ and buffer used for measurements of BfEAS.

\begin{tabular}{cc}
\hline buffer & pH range \\
\hline Citrate-Phosphate & $2.82-7.25$ \\
Phosphate & $5.5-8.0$ \\
Tris & $7.31-9.22$ \\
Glycine-NaOH & $8.71-9.9$ \\
\hline
\end{tabular}

\subsubsection{Spectroscopic analysis}

\subsubsection{UV/vis spectroscopy}

UV/vis spectra were performed in $50 \mathrm{mM}$ sodium phosphate buffer, $\mathrm{pH}$ 8.0 (PpHPL and BfEAS) or in $100 \mathrm{mM}$ sodium phosphate buffer, $\mathrm{pH} 6.0$ (PpAOS1 and PpAOS2) at RT in the range of $650 \mathrm{~nm}$ to $250 \mathrm{~nm}$. It was measured with a Varian Cary 100 Bio UV/vis Spectrophotometer (Varian Inc., Palo Alto, CA, USA).

\subsubsection{C $\quad$ ircularDichroism (CD) spectroscopy}

With the CD spectroscopy method the optical activity of chiral molecules in solution can be analyzed. The CD spectra shows how circularly polarized light can be absorbed by optically active substances. Therefore information about the native folding of a protein can be obtained.

$\mathrm{CD}$ spectra were performed in $50 \mathrm{mM}$ or $100 \mathrm{mM}$ sodium phosphate buffer, $\mathrm{pH} 8.0$ in the range of $260 \mathrm{~nm}$ to $190 \mathrm{~nm}$ on a Chirascan ${ }^{\mathrm{TM}}$ CD Spectrometer (Applied Photophyscics Ltd., Leatherhead, United Kingdom). 


\section{Results}

The aim of the study was the biochemical characterization of the CYP74-enzymes in the moss Physcomitrella patens. P. patens is a model organism and a special feature of the moss is that it contains additionally to the $\mathrm{C}_{18}$ fatty acids LA and LeA a large amount of the $\mathrm{C}_{20}$ fatty acids ARA and EPA (Dembitsky, 1993, Girke et al., 1998) which can also be used as substrates for the oxylipin biosynthesis (Wichard et al., 2005).

In addition to gather insights into CYP74-catalysis and to identify factors determining product specificity the EAS of the lancelet Branchiostoma floridae was also characterized. B. floridae contains various fatty acids as potential precursors of EAS substrates. Beside the $C_{18}$ fatty acids it contains the $C_{22}$ fatty acid docosahexaenoic acid (Lee et al., 2008).

For characterization substrate specificity against hydroperoxides derived from $\mathrm{C}_{18}$ - and $\mathrm{C}_{22}$ fatty acids were determined. Spectral analysis, $\mathrm{pH}$ optima as well kinetic parameters for different substrates were determined. Product formation was analyzed by radio-HPLC and RP-HPLC/MS ${ }^{2}$. Additionally site-directed mutagenesis was carried out in order to identify amino acid residues that are essential for a distinctive EAS activity.

In order to compare the CYP74-enzyme EAS with the CYP74-enzymes from Physcomitrella the substrate specificity against the hydroperoxides from the $C_{18}$ fatty acids LA, $\alpha$-LeA, and $\gamma$-LeA, and the $\mathrm{C}_{20}$ fatty acid ARA were determined. Furthermore spectral analysis, the $\mathrm{pH}$ optima as well the kinetic parameters $\mathrm{K}_{\mathrm{M}}$ and $\mathrm{V}_{\max }$ for the different substrates were determined. The formed products were analyzed by radio-HPLC. Also site-directed mutagenesis was carried out and analyzed.

\subsection{Biochemical characterization of CYP74-enzymes from P. patens}

\subsubsection{Cloning and expression}

The plasmids have previously been constructed as part of former studies (Table 3.1).The two AOS genes as well as the two DES genes were cloned with $\mathrm{N}$-terminal hexahistidine peptide, whereas the HPL was cloned with a C-terminal hexahistidine peptide. In PpHPL the chloroplastidial signal peptide was cut off by truncation of 42 amino acids at the $\mathrm{N}$-terminal. Than an additionally amino acid sequence, MAKKTSS (methionine-alanine-lysine-lysine-threonine-serine-serine) was added at the $\mathrm{N}$ terminal to improve the solubility and the amount of protein. This additional sequence was successfully used before for the purification of AtAOS (Lee et al., 2008). 
Table 3.1: Expression vectors used in this study.

\begin{tabular}{|c|c|c|}
\hline cDNA & vector & $\mathrm{N}$ - or $\mathrm{C}$-terminal amino acid sequence \\
\hline PpAOS1* & pQE30 & MRGSHHHHHHGSACM \\
\hline PpAOS2 ${ }^{\#}$ & pET28a & MGSSHHHHHHSSGLVPRGSHM \\
\hline PpHPL & pET24b & $\begin{array}{c}\text { DVNKLAAALEHHHHHH } \\
\text { MAKKTSSR(43) }\end{array}$ \\
\hline StDES* & pQE30 & MRGSHHHHHHGSM \\
\hline AsDES* & pQE30 & MRGSHHHHHHGSM \\
\hline
\end{tabular}

*cloned by Dr. Michael Stumpe "cloned by Dr. Ellen Hornung

The transitions between the $\mathrm{C}$ - or $\mathrm{N}$-terminal His-tag and the protein were underlined.

The genes were amplified by PCR and expressed in the E. coli strain BL21 Star (2.3.1 and 2.3.6). Because CYP74-enzymes are heme containing enzymes, $80 \mathrm{mg} / \mathrm{L} \delta$-amino levulinoic acid and $150 \mu \mathrm{M}$ $0.1 \mathrm{mM}$ ammonium ferric citrate as cofactors were added. The amino levulinoic acid is a precursor in the heme biosynthesis-pathway and the ferric citrate serves as iron source for the heme.

\subsubsection{Purification}

The purification of the His-tagged proteins was done according to a previously established purification protocol (diploma thesis Scholz, 2009). Cells were prepared according to the membrane preparation method reported by Richardson (Richardson et al., 1993) which is used for the isolation of P450-enzymes from membranes with some minor modifications (2.3.7). After membrane preparation the protein was purified by affinity chromatography.

Proteins were loaded onto a $1 \mathrm{ml}$ His-trap column and then washed with 20 volumes of binding buffer. Non- and unspecifically bound proteins were washed from the column with 20 volumes of elution buffer containing $15 \mathrm{mM}$ imidazole. Finally the bound protein was directly eluted with $300 \mathrm{mM}$ imidazole. Collected fractions were tested for activity (2.3.11.1) and analyzed by SDS-PAGE (2.3.9). Figure 3.1 shows the SDS-PAGE of the purification of PpAOS1. The fractions of the eluted protein showed a significant enrichment, but were not very pure, because they still showed in addition to the strong PpAOS1 band at $55 \mathrm{kDa}$ also further protein bands at $30 \mathrm{kDa}, 50 \mathrm{kDa}, 66 \mathrm{kDa}$, $95 \mathrm{kDa}$ and $105 \mathrm{kDa}$. 


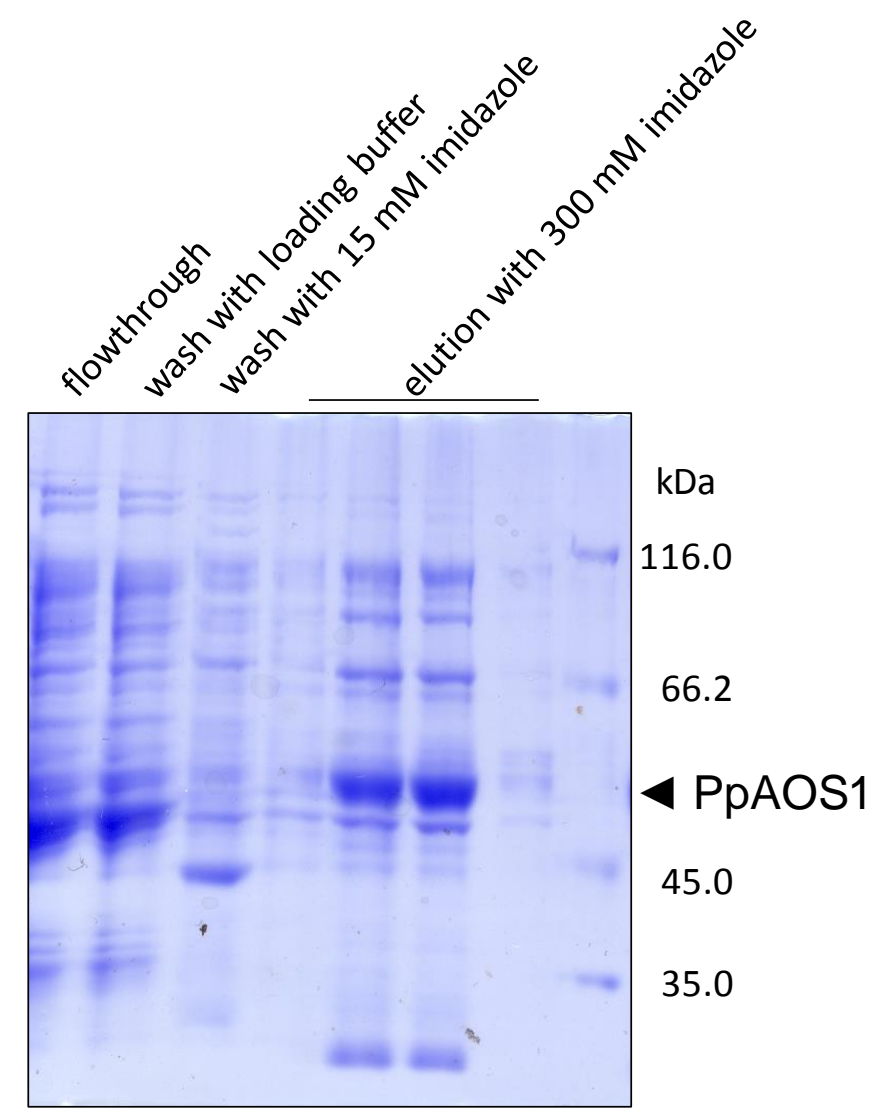

Figure 3.1: SDS-PAGE analysis of PpAOS1purification by stepwise elution. PpAOS1 was heterologously expressed in BL21 Star. The cells were harvested and disrupted by sonification and the cell debris was removed by centrifugation. The purification was done by $\mathrm{Ni}^{2+}$-affinity chromatography. First the column was washed with binding buffer ( $50 \mathrm{mM}$ sodium phosphate, $\mathrm{pH} 8.0,1 \mathrm{M} \mathrm{NaCl}, 500 \mathrm{mM}$ urea) and then with buffer which contains additionally $15 \mathrm{mM}$ imidazole. The protein was directly eluted from the column with $300 \mathrm{mM}$ imidazole. Aliquots of collected fractions of the purification were loaded on a $10 \%$ SDS-PAGE gel. The figure is representative for at least three purifications.

In this work further modifications were done in order to optimize the purification procedure: e. g. the elution of the protein was performed by employing a linear gradient with an increasing imidazole concentration (15 $\mathrm{mM}$ to $300 \mathrm{mM}$ ) to elute unspecifically bound proteins. The SDS-PAGE for the PpAOS1 purification using this gradient (Figure 3.2) showed that the eluted protein was more homogenous after these modifications. Thus the gradual elution led to an optimized purification of the protein compared to the stepwise elution. 


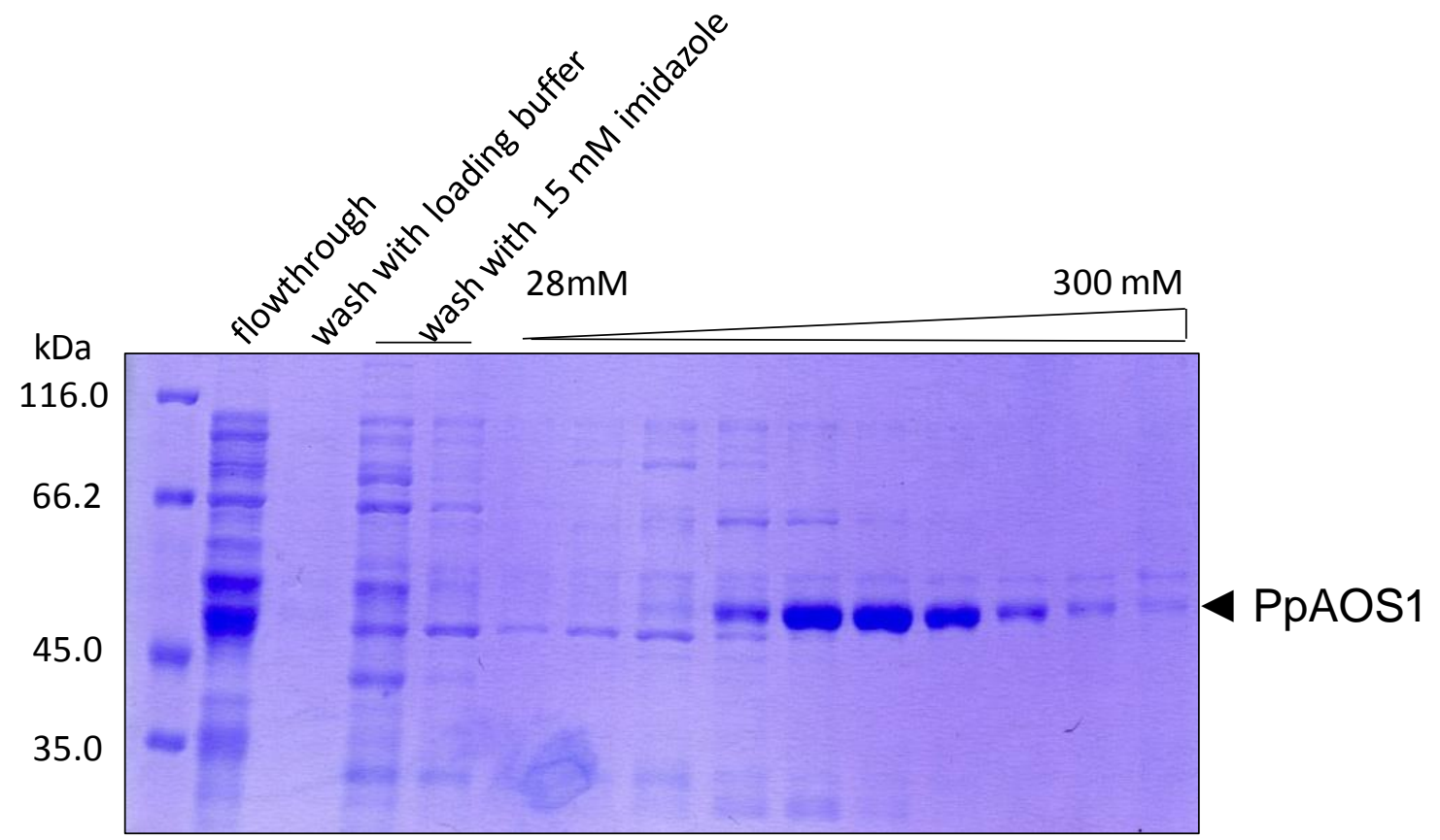

Figure 3.2: SDS-PAGE analysis of PpAOS1purification by gradual elution. PpAOS1 was heterologously expressed in BL21 Star. The cells were harvested and disrupted by sonification and the cell debris was removed by centrifugation. The purification was done by $\mathrm{Ni}^{2+}$-affinity chromatography. First the column was washed with binding buffer ( $50 \mathrm{mM}$ sodium phosphate, $\mathrm{pH} 8.0,1 \mathrm{M} \mathrm{NaCl}, 500 \mathrm{mM}$ urea) and then with buffer which contains additionally $15 \mathrm{mM}$ imidazole. The protein was eluted from the column by a linear gradient with an increasing imidazole concentration ( $15 \mathrm{mM}$ to $300 \mathrm{mM}$ ). Aliquots of collected fractions of the purification were loaded on a $10 \%$ SDS-PAGE gel. The figure is representative for at least three purifications.

In Figure 3.3 the SDS-PAGE analyses of the purification of PpHPL (A and B) and PpAOS2 (C) by stepwise elution ( $A$ ) and by gradual elution ( $B$ and $C$ ) are shown. Both enzymes were also purified more homogenous by elution with an imidazole gradient than by stepwise elution with imidazole.

By using these modified purification procedures it was possible to obtain approx. between 5-15 mg protein/ml expression cultures. The protein was highly enriched and homogenous and was used for further analysis. 

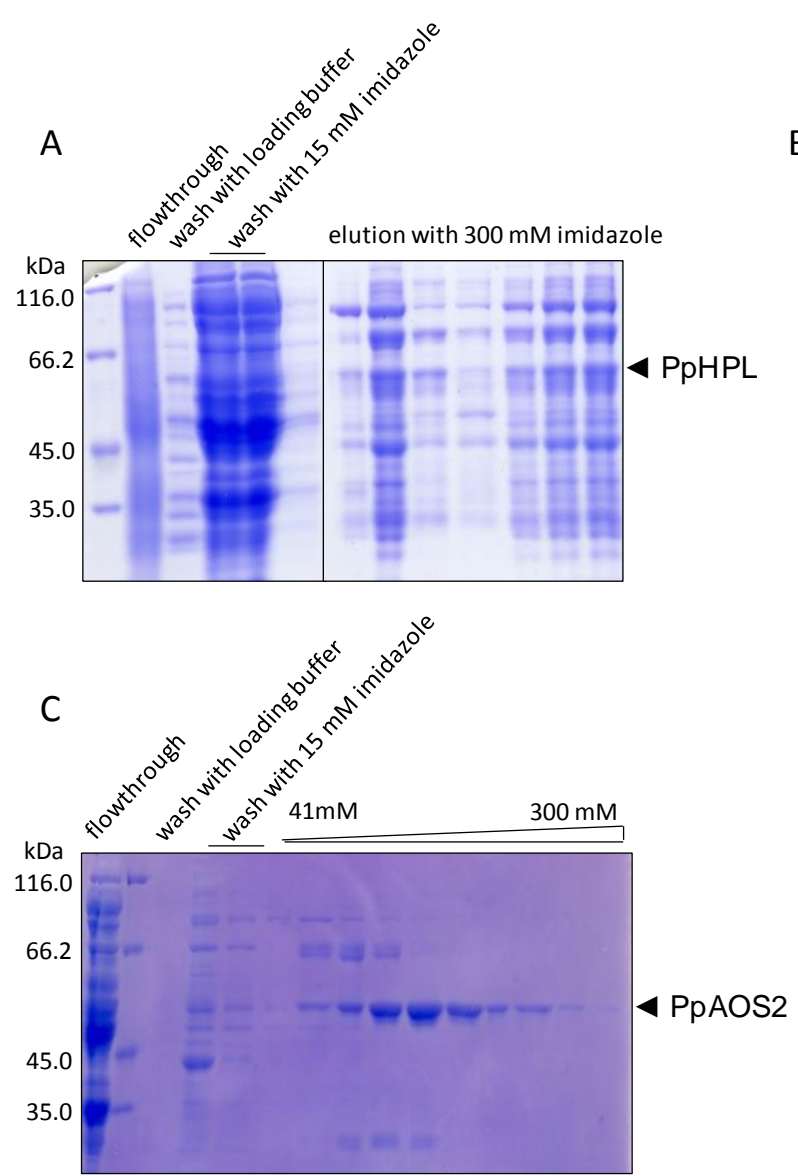

B

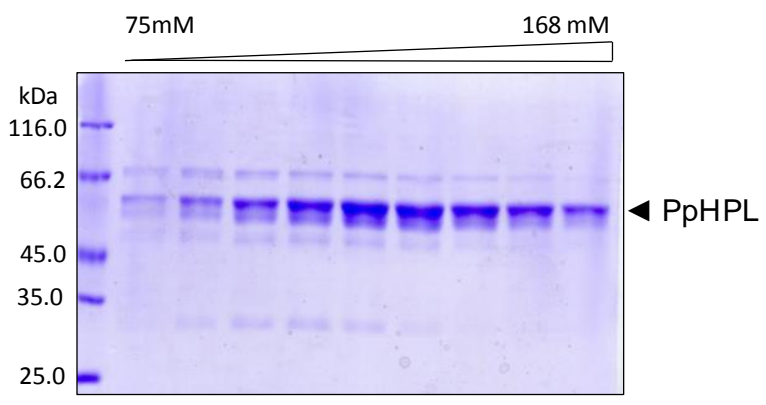

Figure 3.3: SDS-PAGE analysis of PpHPL (A, B) and PpAOS2 (C) purification.The enzymes were heterologously expressed in BL21 Star. The cells were harvested and disrupted by sonification and the cell debris were removed by centrifugation. The purification was done by $\mathrm{Ni}^{2+}$-affinity chromatography. First the column was washed with binding buffer ( $50 \mathrm{mM}$ sodium phosphate, $\mathrm{pH} 8.0,1 \mathrm{M} \mathrm{NaCl}, 500 \mathrm{mM}$ urea) and then with buffer which contains additionally $15 \mathrm{mM}$ imidazole. The protein was directly eluted from the column with $300 \mathrm{mM}$ imidazole $(A)$ or it was eluted by a linear gradient with an increasing imidazole concentration (15 mM to $300 \mathrm{mM})(\mathrm{B}, \mathrm{C})$. Aliquots of collected fractions of the purification were loaded on a $10 \%$ SDS-PAGE gel.The figure is representative for at least three purifications.

By size exclusion chromatography the native molecular weight of the proteins can be determined. In this method the proteins were separated according to their size. The protein mix interacts with the dextrane matrix of the column, whereas the smaller molecules can be retained longer by the matrix than the bigger ones.

SDS-PAGE analysis of PpHPL showed a molecular weight of $57 \mathrm{kDa}$ (Figure $3.3 \mathrm{~A}$ and B) and the theoretical calculated mass was of $57 \mathrm{kDa}$. Analysis by size exclusion chromatography (data not shown) showed that PpHPL has under the conditions applied a native molecular weight of $113 \mathrm{kDa}$ and was therefore 2 -fold higher than the theoretical calculated weight suggesting that PpHPL is a homodimeric protein complex.

PpAOS1 (Figure 3.2) and PpAOS2 (Figure 3.3C) showed on SDS-PAGE analysis molecular weights of $55 \mathrm{kDa}$ which is in agreement with the theoretical calculated masses of $55 \mathrm{kDa}$. The analysis by size exclusion chromatography showed a native molecular weight of $40 \mathrm{kDa}$ for PpAOS1 which was similar to the theoretical mass and therefore PpAOS1 is a monomer (data not shown). The native 
weight of PpAOS2 was determined to be $240 \mathrm{kDa}$ and therefore about 4.5 -fold higher than the theoretical weight indicating that PpAOS2 might be a tetrameric or pentameric protein complex.

\subsubsection{Spectroscopic analysis}

\subsubsection{UV/vis analysis}

The protein fractions obtained by affinity chromatography showed reddish (PpHPL and PpAOS1) and yellowish (PpAOS2) colours, which indicated the presence of a heme cofactor. To prove and determine the heme content UV/vis spectra of the purified enzymes were recorded in $50 \mathrm{mM}$ sodium phosphate buffer, pH 8.0 (PpHPL) and in 100 mM sodium phosphate buffer, pH 6.0 (PpAOS1 and PpAOS2), respectively, at RT in the range of $650 \mathrm{~nm}$ to $250 \mathrm{~nm}$ (2.3.15.1).

Heme containing proteins absorb in visible light and have characteristic absorption maxima (Lottspeich, 2006). The UV/vis spectrum of PpAOS1 in Figure 3.4 A showed these characteristic maxima. The Soret-band had an absorption maximum at $415 \mathrm{~nm}$. Further maxima were at $280 \mathrm{~nm}\left(\gamma^{-}\right.$ band) and $535 \mathrm{~nm}$ ( $\beta$-band).

The spectrum of PpAOS2 (Figure $3.4 \mathrm{~B}$ ) is similar to that of PpAOS1 with a Soret-band at $411 \mathrm{~nm}, \mathrm{\gamma}$ band at $280 \mathrm{~nm}$, and the $\beta$-band at $529 \mathrm{~nm}$.

PpHPL (Figure $3.4 \mathrm{C}$ ) showed absorption maxima at $415 \mathrm{~nm}$ (Soret-band) and $280 \mathrm{~nm}$ ( $\gamma$-band), and smaller maxima at $540 \mathrm{~nm}$ ( $\beta$-band), $360 \mathrm{~nm}$ ( $\delta$-band), and $570 \mathrm{~nm}$ ( $\alpha$-band). 
A

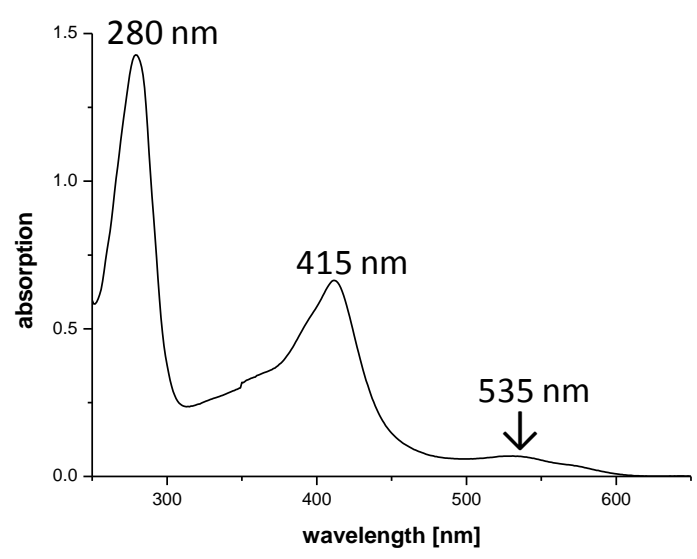

C

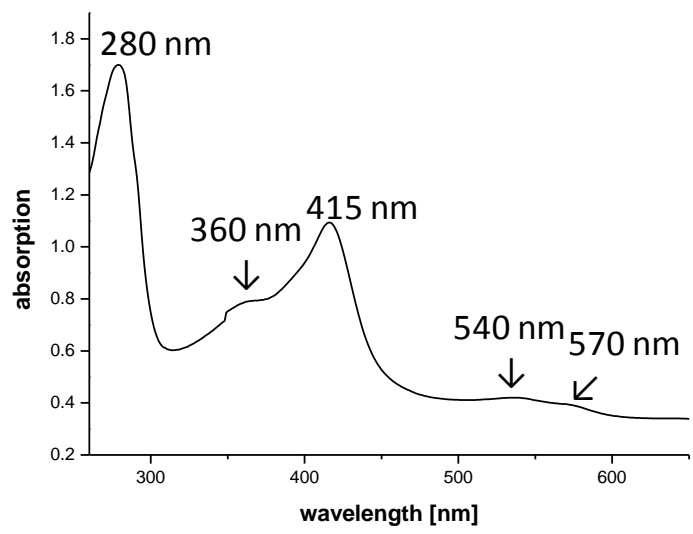

B

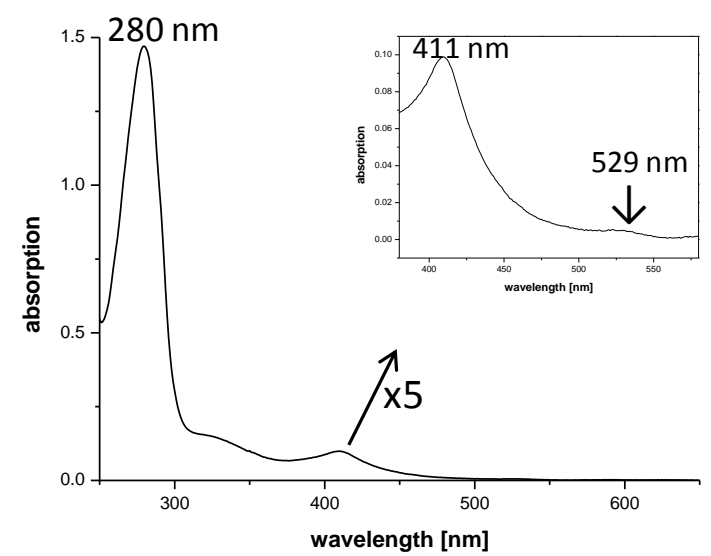

Figure 3.4: UV/vis spectra of purified PpAOS1 (A), PpAOS2 (B) and PpHPL (C). They were measuredin $100 \mathrm{mMsodium}$ phosphate buffer, $\mathrm{pH} 6.0$ (A and B) or $50 \mathrm{mMsodium}$ phosphate buffer, $\mathrm{pH} 8.0$ (C), respectively. Note that spectrum A and B were adapted from a former study (diploma thesis Scholz, 2009) and are shown for reasons of comparison. The spectra are representative for at least two experiments.

The heme content of those enzymes varies significantly: Based on theoretical molar extinction coefficients (calculated with the Protparam-software tool (http://web.expasy.org/protparam/l) at $280 \mathrm{~nm}\left(\varepsilon_{280}(\mathrm{PpAOS} 1) \approx 62.000 \mathrm{M}^{-1} \mathrm{~cm}^{-1}\right.$ and $\left.\varepsilon_{280}(\mathrm{PpAOS} 2) \approx 57.000 \mathrm{M}^{-1} \mathrm{~cm}^{-1}\right)$ and an excepted molar extinction coefficient for the Soret-band of $\varepsilon_{\text {soret }} \approx 100.000 \mathrm{M}^{-1} \mathrm{~cm}^{-1}$, the calculated heme content of PpAOS1 is of approx. $30 \%$ and of PpAOS2 only $4 \%$ PpHPL $\left(\varepsilon_{280}(\mathrm{PpHPL}) \approx 71.000 \mathrm{M}^{-1} \mathrm{~cm}^{-1}\right)$ had a calculated heme content of $46 \%$.

All spectra did not show signals at $630 \mathrm{~nm}$, which are characteristic for high-spin iron-III-complexes. Therefore the hemeiron in the active site is apparently in its low-spin state, which is characteristic for P450 ezymes.

\subsubsection{CD spectroscopy}

To analyze the native protein folding of the proteins $C D$ spectra were measured. The spectra were recorded in $50 \mathrm{mM}$ or $100 \mathrm{mM}$ sodium phosphate buffer, $\mathrm{pH} 8.0$, respectively, in the range of $260 \mathrm{~nm}$ to $190 \mathrm{~nm}$ (2.3.15.2). In Figure 3.5 the spectrum of purified PpHPL is shown, there were negative 
peaks at $215 \mathrm{~nm}$ and $220 \mathrm{~nm}$, and a positive peak at $195 \mathrm{~nm}$. These peaks are typical for proteins with a mainly $\alpha$-helical fold (Panagakou et al., 2012).

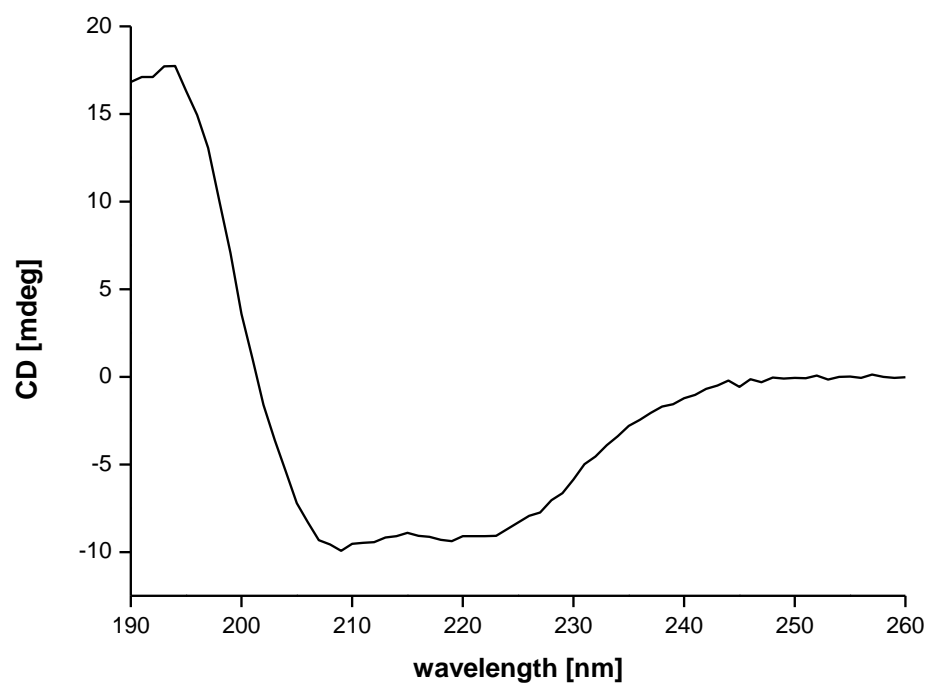

Figure 3.5: CD spectrum of purified PpHPL. It was measured in $100 \mathrm{mM}$ sodium phosphate buffer, $\mathrm{pH} 8.0$, on a Chirascan $^{\mathrm{TM}}$ CD Spectrometer (Applied Photophysics Ltd., Leatherhead, United Kingdom). The spectrum was recorded in the range of $260 \mathrm{~nm}$ to $190 \mathrm{~nm}$. The figure shows the spectrum of oneexperiment.

PpAOS1 showed negative peaks at $215 \mathrm{~nm}$ and $222 \mathrm{~nm}$, and a positive peak at $193 \mathrm{~nm}$ (Figure 3.6).

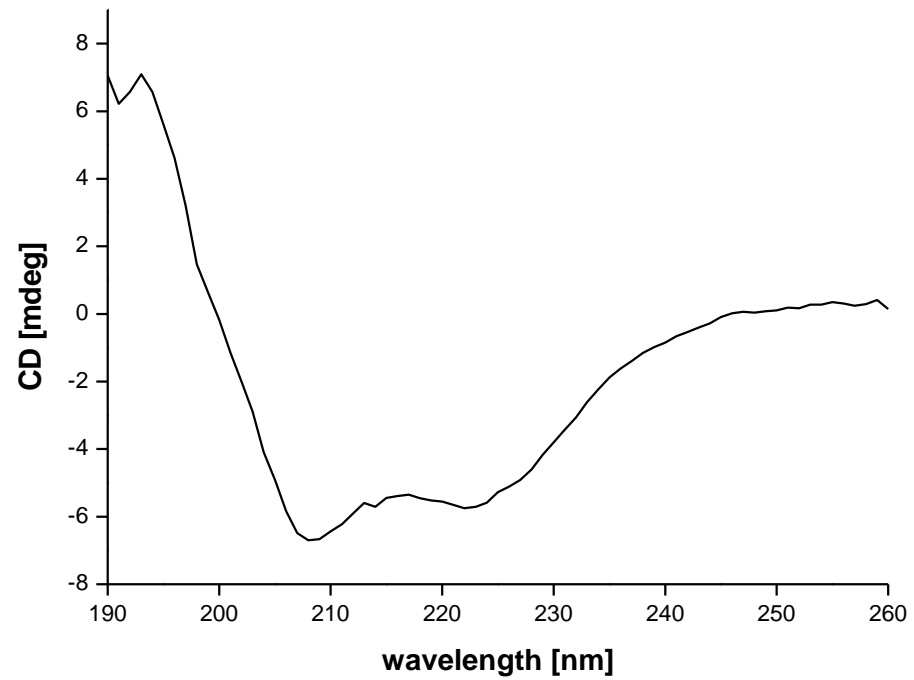

Figure 3.6: $\mathrm{CD}$ spectrum of purified PpAOS1. It was measured in $50 \mathrm{mM}$ sodium phosphate buffer, $\mathrm{pH} 8.0$ on a Chirascan $^{\mathrm{TM}}$ CD Spectrometer (Applied Photophysics Ltd., Leatherhead, United Kingdom). The spectrum was recorded in the range of $260 \mathrm{~nm}$ to $190 \mathrm{~nm}$. The figure shows the spectrum of one experiment. 


\subsubsection{Analysis of products}

\subsubsection{Radio-HPLC}

Reaction products formed by incubations of different $\left[1-{ }^{14} \mathrm{C}\right]$-hydroperoxy fatty acid derivatives with PpAOS1, PpAOS2 and PpHPL were analyzed by means of radio-HPLC. In this method the different products were separated via RP-HPLC (2.3.13.1) and detected by using a scintillation detector which was connected to the chromatographic system. The enzymatic conversions were performed with purified PpAOS1 and PpHPL with 9- and 13 -hydroperoxides derived from $\left[1-{ }^{14} \mathrm{C}\right]$-labeled LA and $\alpha$-LeA as substrates (2.3.10.4). The reaction mix was incubated and the products were extracted with diethyl ether as described (2.3.13).

Exemplarily shown in Figure 3.7 are the radio-RP-HPLC analyses of products formed by incubation of PpAOS1 and PpHPL, respectively, with 9-HPOD. In analogy, the respective analysis of products formed from incubation of 13-HPOT with PpAOS1 and PpHPL is exemplarily shown in Figure 3.8.

The reaction of PpAOS1 with $\left[1-{ }^{14} \mathrm{C}\right]$-labeled $9-\mathrm{HPOD}$ led to the formation of various products. These products were identified as $\alpha$ - (RT $7.5 \mathrm{~min}$; main product) and $\gamma$-ketol (RT $5 \mathrm{~min}$ ), and as 9-oxo nonanoic acid (RT $2 \mathrm{~min}$ ). The ketols arise from the non-enzymatically hydrolysis of the unstable allene oxide. The detected 9-oxo nonanoic acid is a distinctive HPLproduct. The main product of the conversion of 9-HPOD by PpHPL was 9-oxo nonanoic (RT $2 \mathrm{~min}$ ) but also different side products as $\alpha$ and $\mathrm{y}$-ketols (RT $7.5 \mathrm{~min}$ and RT $5 \mathrm{~min}$ ) were formed. As control buffer was incubated with substrate. The chromatograms for the conversions of $9 \alpha$-HPOT (see appendixFigure $9.1 \mathrm{~A}$ ) were similar to that of 9-HPOD. 


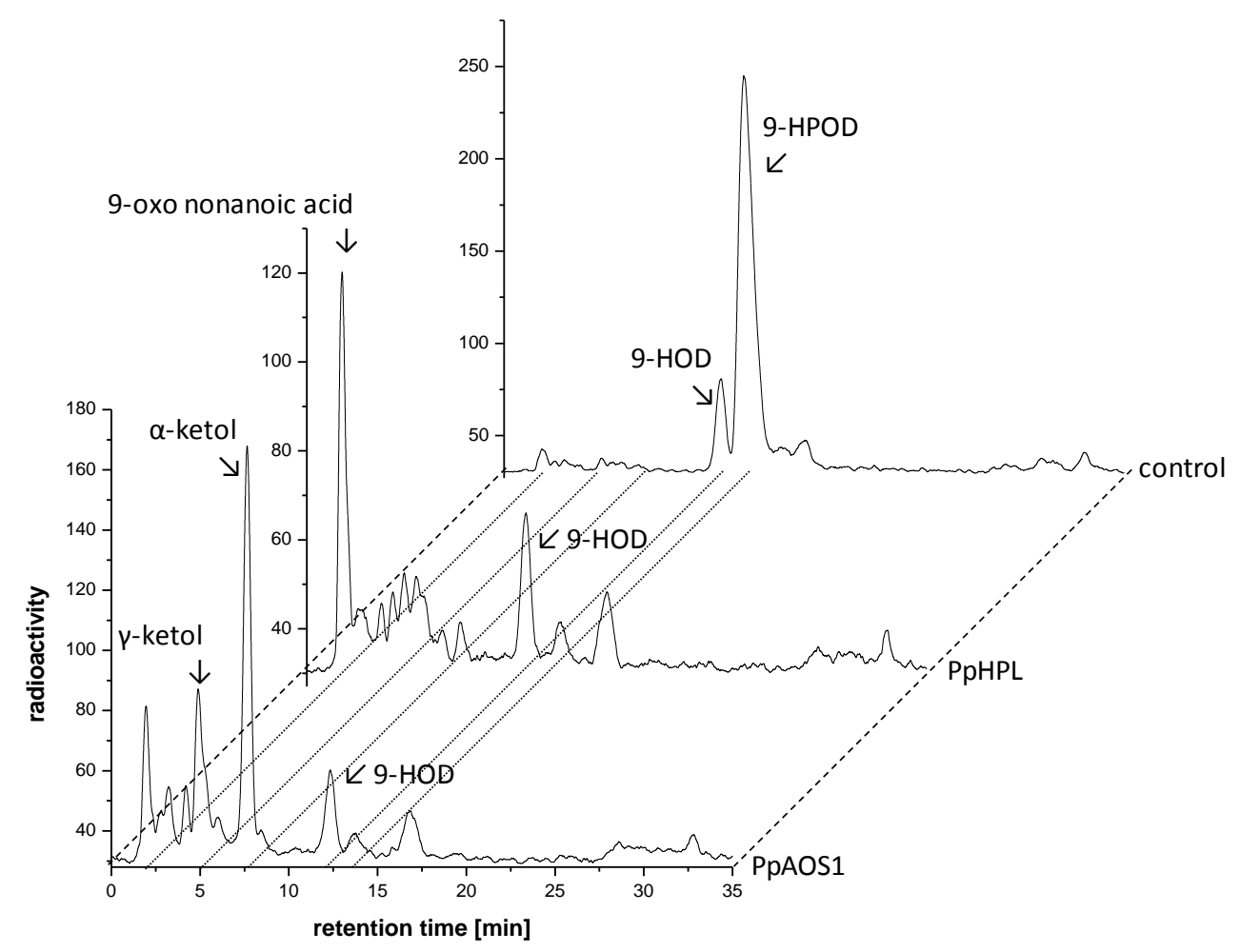

Figure 3.7: Product analysis of PpAOS1 and PpHPL with $\left[1-{ }^{14} \mathrm{C}\right]-9-\mathrm{HPOD}$. The purified enzymes as well as only buffer (control) were incubated with the radio-labeled substrate for approx. $30 \mathrm{~min}$ at RT. After extraction with diethyl ether the products were analyzed by RP-HPLC coupled to a radio-detector. The chromatograms are representative for three experiments.

In Figure 3.8 the chromatograms for the reactions of radio-labeled $13 \alpha$-HPOT are shown. The conversion of $13 \alpha$-HPOT by PpHPL yielded (9Z)-12-oxo-9-dodecenoic acid (RT $2.5 \mathrm{~min}$ ) as main product. As for the conversion of the respective 9-hydroperoxy fatty acids also incubation of PpAOS1 with $13 \alpha$-HPOT yielded a considerable amount of this HPLproduct. However, the main product was $\alpha$-ketol (RT $5.5 \mathrm{~min}$ ). In addition small amounts of the respective $\gamma$-ketol (RT $3.5 \mathrm{~min}$ ) and cyclopentenone (RT $7.5 \mathrm{~min}$ ) derivatives were detected. The latter product is known to arise from the non-enzymatical cyclization of the allene oxide (Hamberg et al., 1988). The peak at about $11 \mathrm{~min}$ could not be identified. The reactions of PpHPL with 13-HPOD as substrate (see appendix Figure 9.1B) gave a similar product pattern as observed with 13 $\alpha$-HPOT as substrate.

The occurrences of both catalytic activities were consistent with a close interconnection of the enzymatic AOS- and HPL-branches as proposed before (Lee et al., 2008, Li et al., 2008,Cho et al., 2011). 


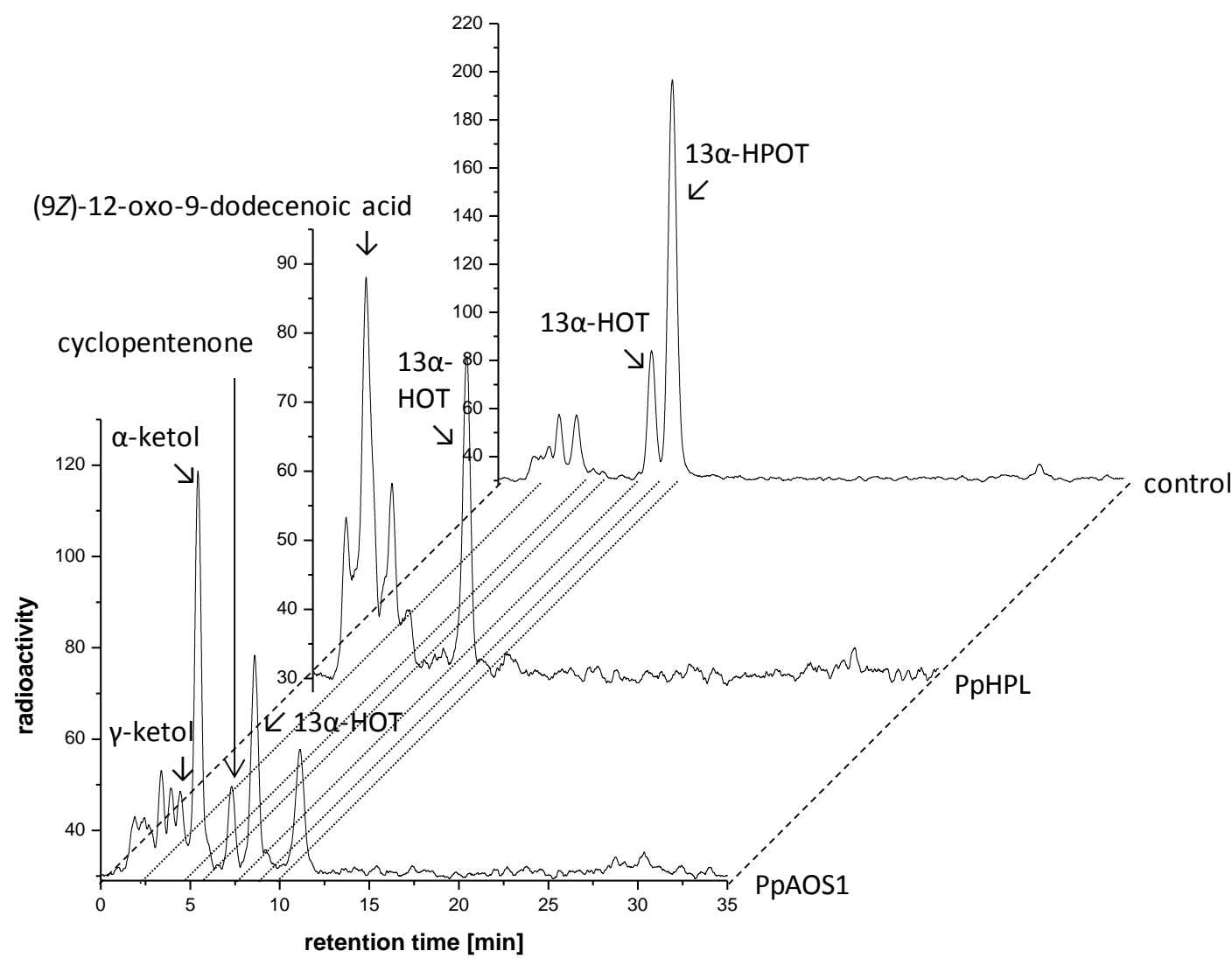

Figure 3.8: Product analysis of PpAOS1 and PpHPL with $\left[1-{ }^{14} \mathrm{C}\right]-13 \alpha-\mathrm{HPOT}$. The purified enzymes as well as only buffer (control) were incubated with the radio-labeled substrate for approx. $30 \mathrm{~min}$ at RT. After extraction with diethyl ether the products were analyzed by RP-HPLC coupled to a radio-detector. The chromatograms are representative for five experiments.

Table 3.2 gives a semi quantitative summary of the products formed by incubations of PpAOS1 and PpHPL with radio-labeled 9- and 13-hydroperoxides derived from LA and $\alpha$-LeA.

Table 3.2: Product specificities of PpHPL and PpAOS1. Affinity purified enzymes were incubated with [1 $\left.{ }^{14} \mathrm{C}\right]-$ labeled hydroperoxy fatty acids for approx. $30 \mathrm{~min}$ at RT. After extraction with diethyl ether the isolated products were analyzed by RP-HPLC that was coupled to a radio-detectorand quantified by integration of the respective peak area. The relative amounts of each product is indicated by the number of " + ".

\begin{tabular}{ccccc}
\hline substrate & enzyme & $\begin{array}{c}\text { w-oxo fatty acids } \\
\text { (= HPL activity) }\end{array}$ & $\begin{array}{c}\text { ketols } \\
\text { (= AOS activity) }\end{array}$ & $\begin{array}{c}\text { cyclopentenone } \\
\text { (= AO cyclization) }\end{array}$ \\
\hline 9-HPOD & HPL & ++++ & + & n. d. \\
\hline 9 $\alpha$-HPOT & AOS1 & + & ++++ n. d. \\
\hline 13-HPOD & HOS1 & ++++ & + & n.d. \\
& HPL & + & ++++ n. d. \\
\hline 13 $\alpha$-HPOT & AOS1 & ++++ n. d. & n.d. \\
\hline
\end{tabular}


The product analysis of PpAOS1 and PpHPL showed that in both enzymes both catalytic activities (AOS and HPL) occurred. This suggests that the branches of both CYP74-enzymes were closely interconnected. To analyze if the DES-branch is also closely interconnected to the AOS- and HPLbranches product analysis of StDES and AsDES were performed. The conversion of $\left[1-{ }^{14} \mathrm{C}\right]-9 \alpha-\mathrm{HPOT}$ by StDES yielded the divinyl ether colnelenic acid (RT $28 \mathrm{~min}$ ) as main product. Also some of the HPLproduct 9-oxo nonanoic acid (RT $2 \mathrm{~min}$ ) was detected (Figure 3.9). The reaction of AsDES with $13 \alpha$-HPOT yielded etherolenic acid as main substrate and also less amounts of (9Z)-12-oxo-9dodecenoic acid as side product (data not shown). Product analysis with 9- and 13-hydroperoxides derived from LA was not performed. Product analysis of DES-enzymes showed that the DES-branch is also closely interconnected to the HPL-branch, because both enzyme catalytic activities occurred in DES.

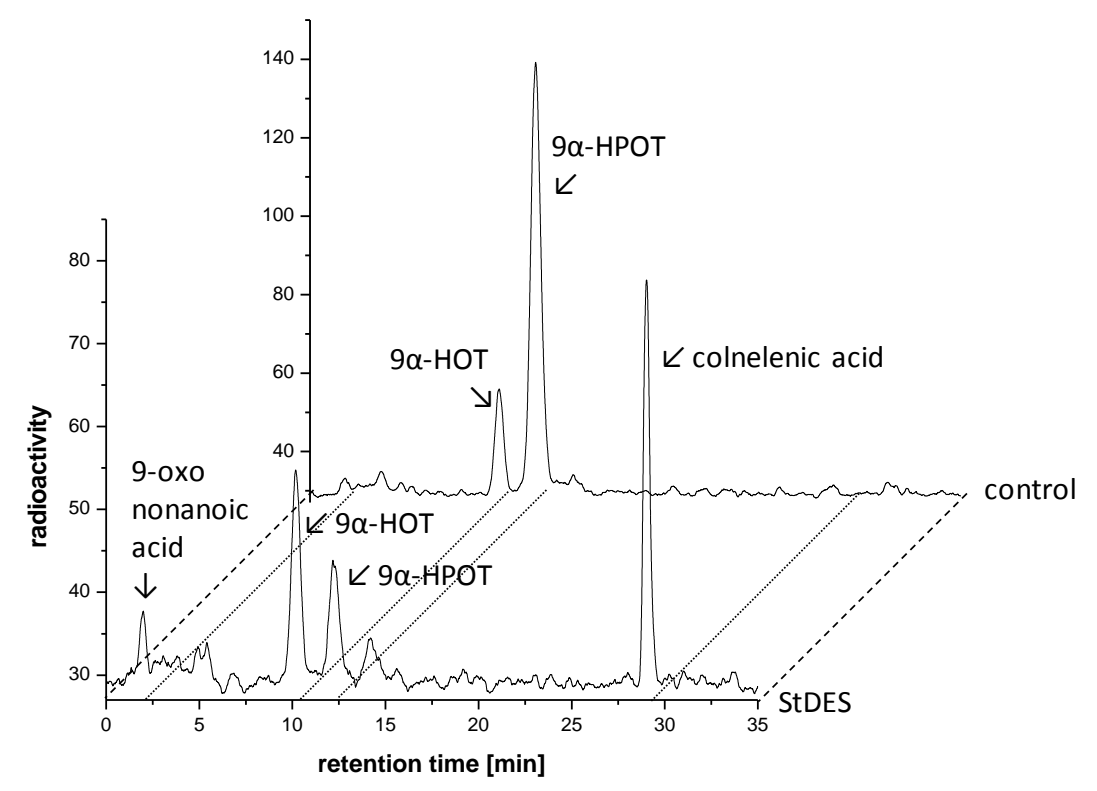

Figure 3.9: Product analysis of StDES with $\left[1-{ }^{14} \mathrm{C}\right]-9 \alpha$-HPOT. The purified enzyme wasas well as only buffer (control) were incubated with the radio-labeled substrate for approx. $30 \mathrm{~min}$ at RT. After extraction with diethyl ether the products were analyzed by RP-HPLC coupled to a radio-detector. The chromatograms are representative for three experiments.

\subsubsection{RP-HPLC/MS ${ }^{2}$}

Next the different products were also analyzed by RP-HPLC/MS ${ }^{2}$. With the radio-HPLC method the different products were solely identified by comparison of the retention times with authentic standards. This method is very sensitive and therefore various sideproducts can be detected. RP$\mathrm{HPLC} / \mathrm{MS}^{2}$ analysis on the other hand gives structural information about the different products. The purified enzymes were incubated with hydroperoxides derived from LA and $\alpha$-LeA, extracted and analyzed as described in 2.3.13.3. The products were first separated by RP-HPLC and subsequently analyzed by ESI-MS. 
In the following figures the extracted ion chromatograms and their associated tandem mass spectra and potential fragment patterns of products from conversion of PpAOS1 with $13 \alpha$-HPOT are shown. For analysis of the products formed by the incubation of PpAOS1 with fatty acid hydroperoxide the respective products were pre-isolated by RP-HPLC/DAD and then analyzed by RP-HPLC/MS ${ }^{2}$. The extracted ion chromatogram $(\mathrm{m} / \mathrm{z} 309)$ and the associated tandem mass spectrum (Figure 3.10$)$ of the collected $\gamma$-ketol fraction of the RP-HPLC/DAD analysis confirmed this product peak as $\gamma$-ketol.

A

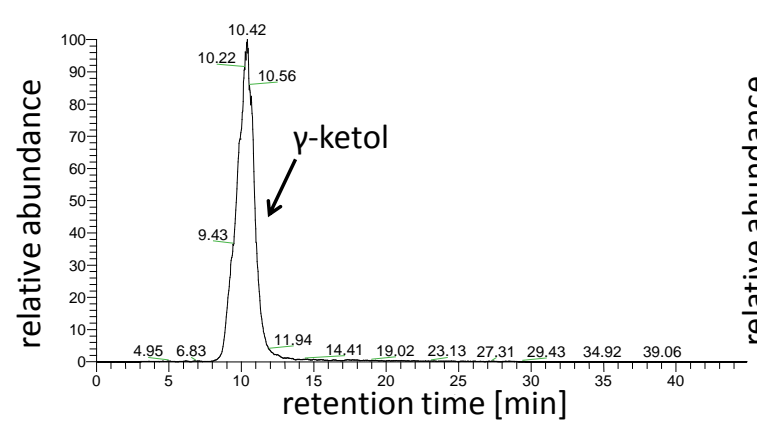

B
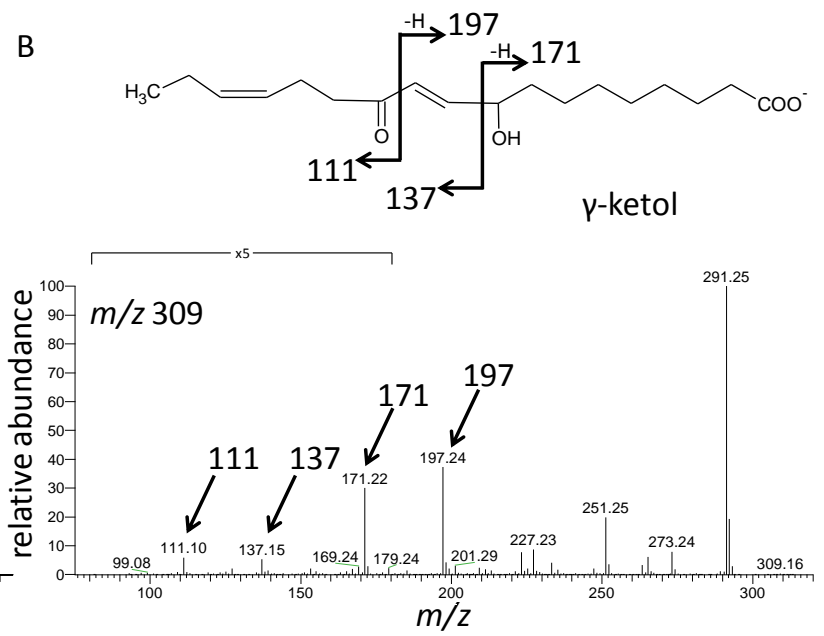

Figure 3.10: RP-HPLC/MS ${ }^{2}$-analysis of products formed by incubation of PpAOS1 with $13 \alpha-\mathrm{HPOT}(\nu$-ketol). Shown are the extracted $\mathrm{m} / \mathrm{z}$ 309ion chromatogram $(\mathrm{A})$ and the associated tandem mass spectrum (B) of thecollected $\gamma$-ketolfraction of the RP-HPLC/DAD analysis.

RP-HPLC/MS ${ }^{2}$ analysis of the collected main product fraction ( $\alpha$-ketol) of the RP-HPLC/DAD analysis confirmed this signal as $\alpha$-ketol, the main product of AOS (Figure 3.11).

A

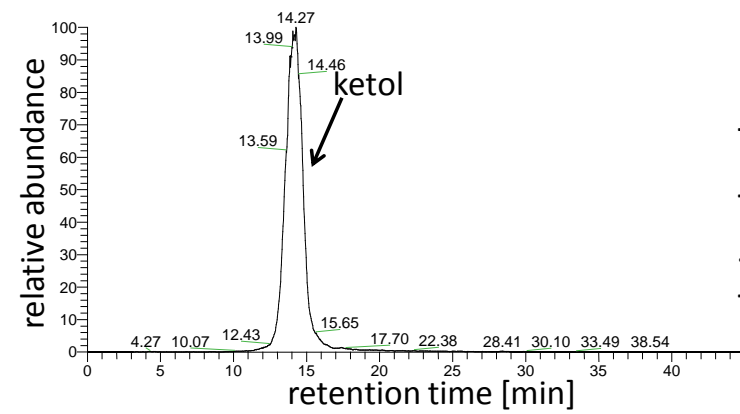

B
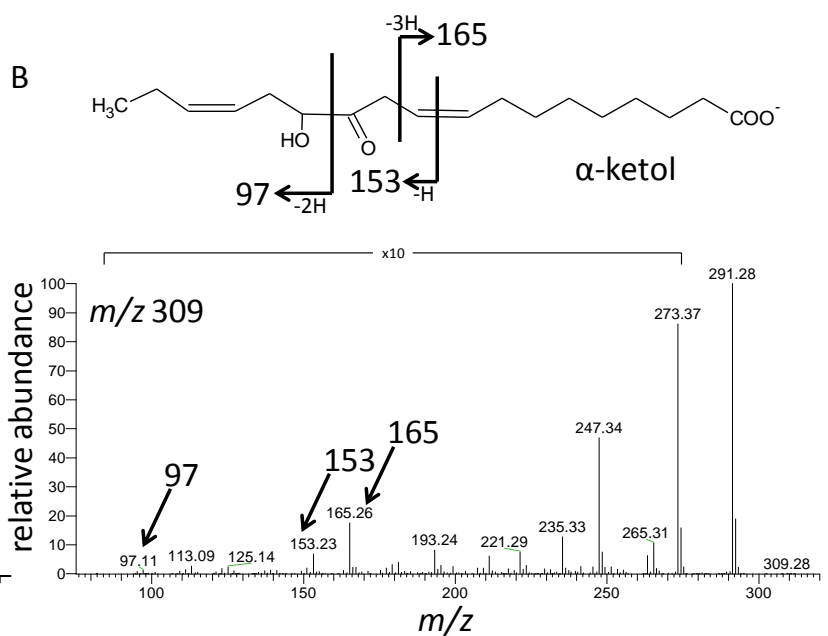

Figure 3.11: RP-HPLC/MS ${ }^{2}$-analysis of products formed by incubation of PpAOS1 with 13 $\alpha$-HPOT ( $\alpha$-ketol). Shown are the extracted $\mathrm{m} / \mathrm{z}$ 309ion chromatogram $(A)$ and the associated tandem mass spectrum (B) of the collected $\alpha$-ketolfraction of the RP-HPLC/DAD analysis.

The $m / z 291$ chromatogram (A) and the tandem mass spectrum (B) shown in Figure 3.12 confirmed the collected cyclopentenone peak of RP-HPLC/DAD analysis as OPDA. 

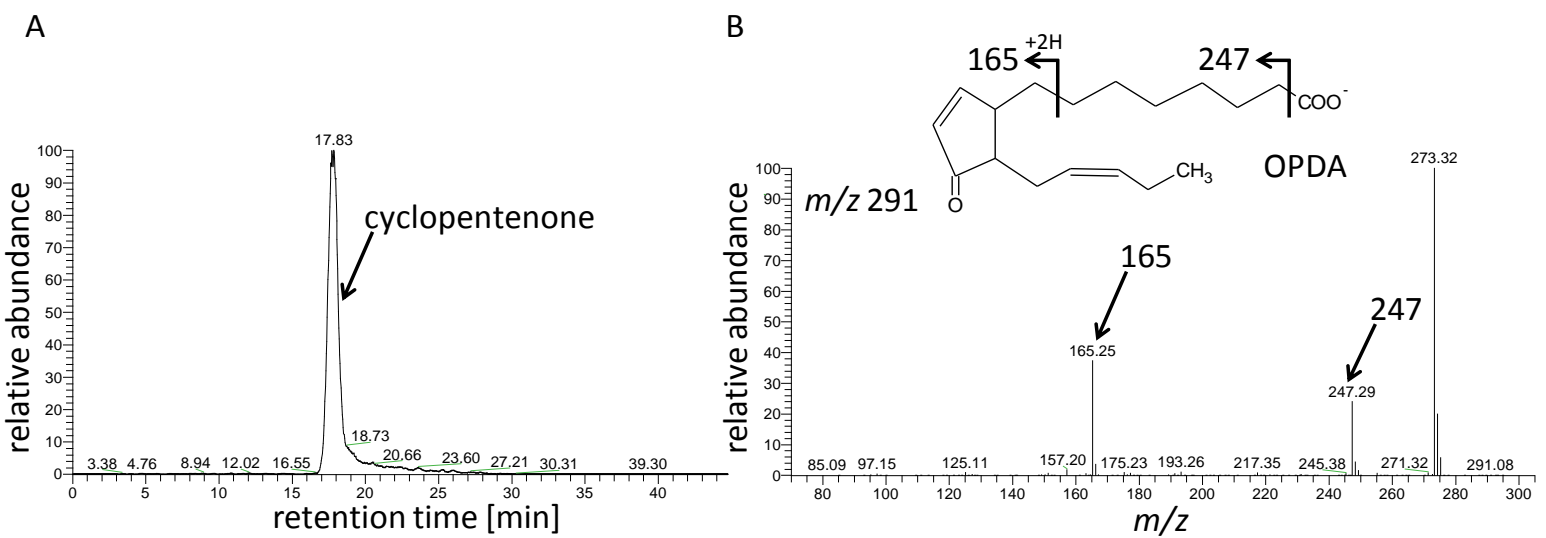

Figure 3.12: RP-HPLC/MS ${ }^{2}$-analysis of products formed by incubation of PpAOS1 with 13 $\alpha$-HPOT (cyclopentenone). Shown are the extracted $\mathrm{m} / \mathrm{z} 291$ ion chromatogram $(A)$ and the associated tandem mass spectrum (B)of the collected cyclopentenonefraction of the RP-HPLC/DAD analysis.

The analysis of incubation of PpHPL with $13 \alpha$-HPOT identified collected product fractions from RPHPLC/DAD analysis as $\alpha$ - (Figure 3.13 A and B)and $\gamma$-ketol (Figure 3.13 $\mathrm{C}$ and D). The extracted ion chromatograms $\mathrm{m} / \mathrm{z} 309$ as well as the tandem mass spectra of the collected products in Figure 3.13were similar to that of PpAOS1 conversions (Figure 3.10 and Figure 3.11).

A

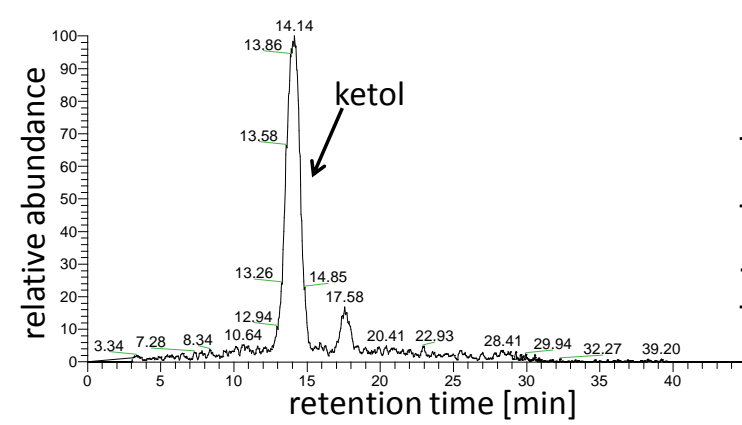

c

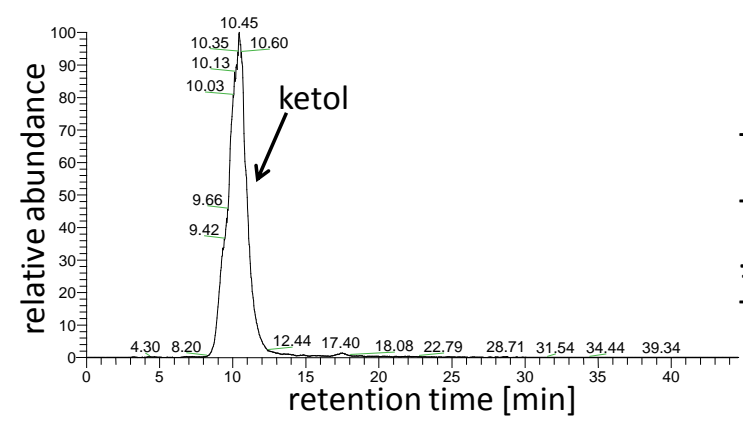

B
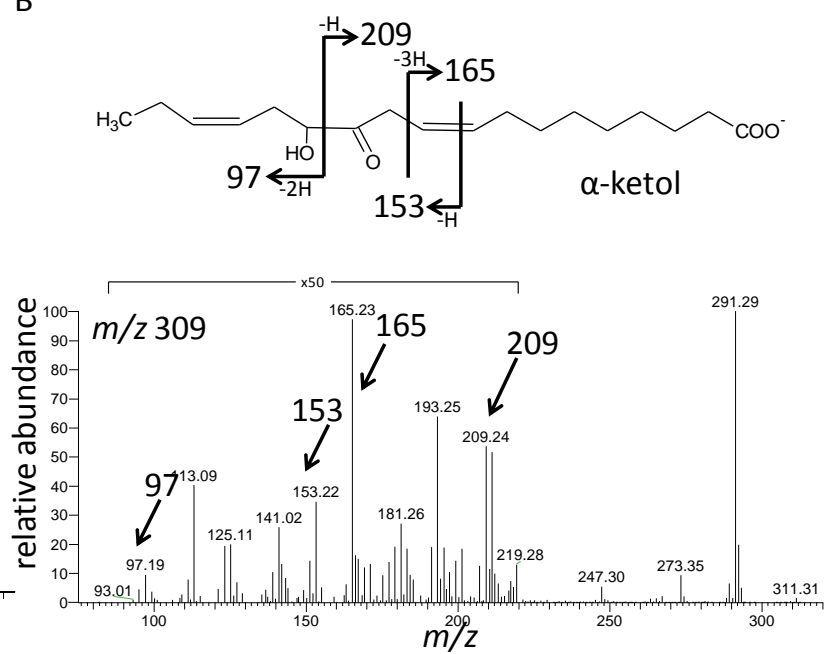

D
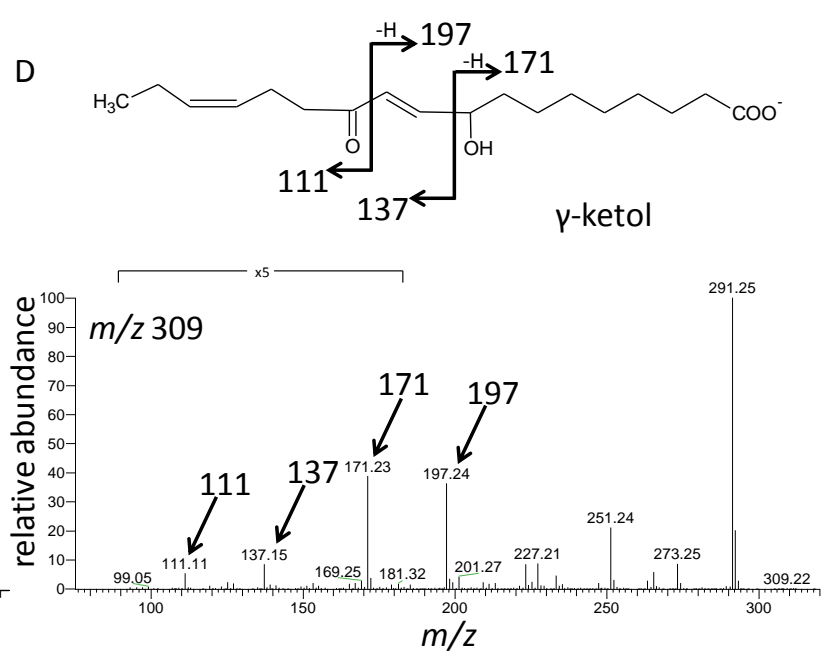
Figure 3.13: RP-HPLC/MS ${ }^{2}$-analysis of products formed by incubation of PpHPL with $13 \alpha$-HPOT ( $\alpha$-and $\gamma$-ketol). Shown are the extracted ion chromatograms $(m / z 309)$ and the associated tandem mass spectra of the collected $\alpha$ - (A and $B$ ) and $\gamma$-ketol (C and D) fractions from the RP-HPLC/DAD analysis.

\subsubsection{Kinetic analysis}

The moss Physcomitrella patens contains $\mathrm{C}_{18^{-}}$and $\mathrm{C}_{20}$ fatty acids. Analysis of kinetic parameters of conversions of PpAOS1 with different fatty acid hydroperoxides derived from LA, $\alpha$-LeA and ARA should determine which substrate is preferred.

Kinetic measurements were done by photometrically approaches as described in 2.3.11.1.

\subsubsection{1 pH optimum}

The determination of the $\mathrm{pH}$ optimum was performed with $0.1 \mathrm{nM}$ PpAOS1 and $30 \mu \mathrm{M} 13-\mathrm{HPOD}$ as described in 2.3.14.1. Therefore the initial velocity for the conversion of the fatty acid hydroperoxide at $234 \mathrm{~nm}$ was measured in different buffers. An acetate buffer was used for the $\mathrm{pH}$ range 4.7 to 5.5 and a phosphate buffer for the range from 5.5 to 8.0.

In Figure 3.14 the initial velocity in dependence on different $\mathrm{pH}$ values is shown. The optimal $\mathrm{pH}$ for PpAOS1 is at $\mathrm{pH} 6.5$.

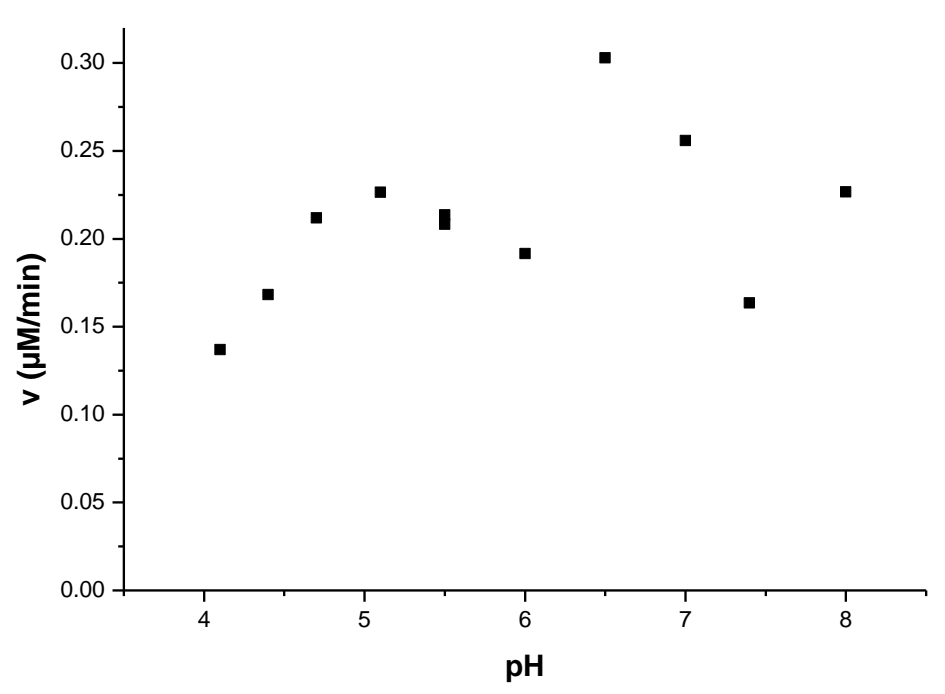

Figure 3.14: The pH optimum of PpAOS1 with 13-HPOD. Measurements of the initial velocity in dependence of different $\mathrm{pH}$ values were carried out with $0.1 \mathrm{nM}$ PpAOS1 and $30 \mu \mathrm{M}$ of 13-HPOD at RT.Each data point represents the mean value of three measurements. 


\subsubsection{Kinetic parameters}

For the determination of the various specificities of PpAOS1 for different substrates the kinetic parameters were measured. Based on the kinetic parameters the different specificities of PpAOS1 and PpAOS2 can be elucidated and compared. Therefore the kinetic parameters $K_{M}$ and $V_{\max }$ for the reaction of PpAOS1 with various hydroperoxides were determined by measuring the initial timedependent substrate consumption at $234 \mathrm{~nm}$ at different substrate concentrations (2.3.11.1). The reactions were performed in sodium phosphate buffer $(50 \mathrm{mM}, \mathrm{pH} 8.0)$ at $\mathrm{RT}$ and were started by the addition of PpAOS1. 9- and 13-hydroperoxides derivatives of LA, $\alpha$-LeA, and $\gamma$-LeA, and 12hydroperoxide derivative of ARA were used as substrates. For the measurements of 9- and 13-HPOD, $9 \alpha$ - and $13 \alpha$-HPOT, and 12-HPETE $0.1 \mathrm{nM} \mathrm{PpAOS1}$ and for the measurements of $9 \gamma$ - and $13 \gamma$-HPOT $0.05 \mathrm{nM}$ PpAOS1 was added. In Figure 3.15 the Michaelis-Menten diagram for the reactions of PpAOS1 with 9- and 13-hydroperoxides derived from the above mentioned $\mathrm{C}_{18}$ fatty acid are shown. It should be noted at this point, that substrate concentration higher than $100 \mu \mathrm{M}$ resulted in an absorption approaching the saturation of the photometer. In order to circumvent this problem the maximal substrate concentration used in standard experiments (with substrates yielding $\mathrm{K}_{\mathrm{M}}<100 \mu \mathrm{M}$ ) was $100 \mu \mathrm{M}$. However, for some substrates, yielding $\mathrm{K}_{\mathrm{M}}>100 \mu \mathrm{M}$, the maximal substrate concentration was $150 \mu \mathrm{M}$. Although this value gives an absorbance higher than the detection limit, it was possible to derive reasonable data from these experiments, since, due to the experimental setup and the resulting dead-time, the substrate was already consumed to some extend when we started to record the absorption after enzyme addition. This normally results in "starting- absorbance" below $3 \mathrm{AU}$. 
A

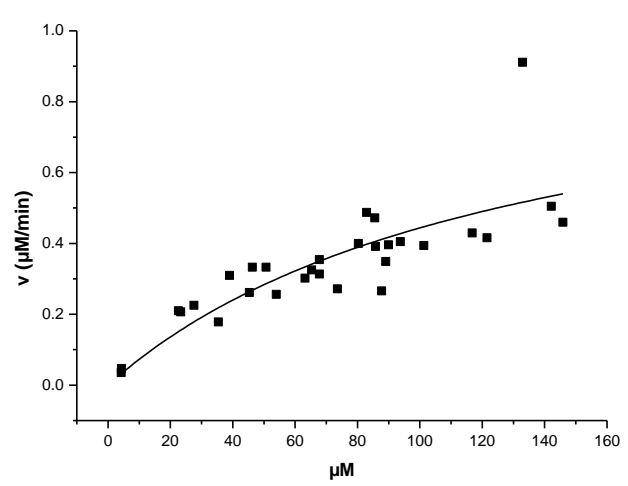

C

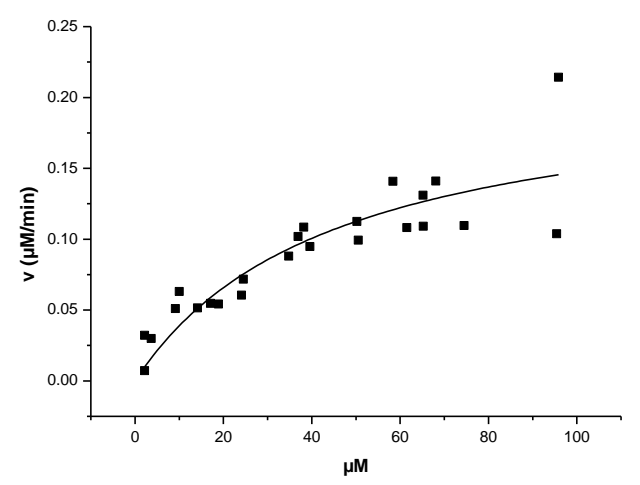

$\mathrm{E}$

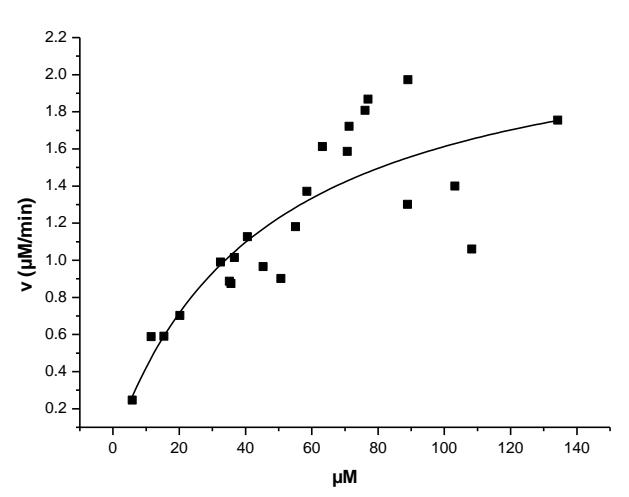

B

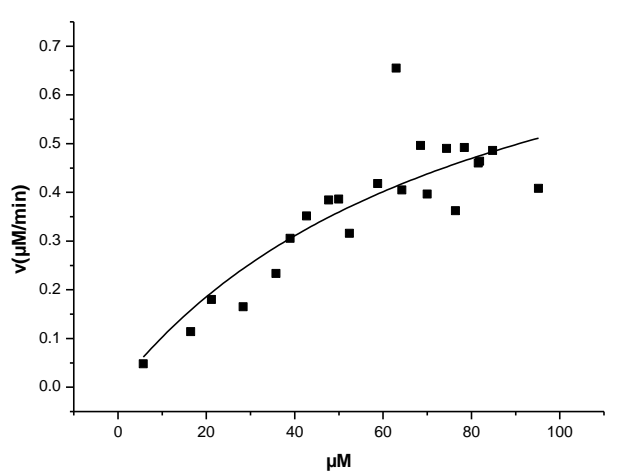

D

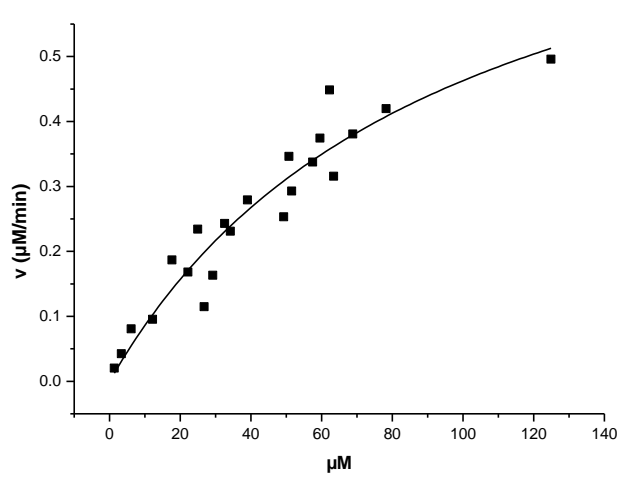

$\mathrm{F}$

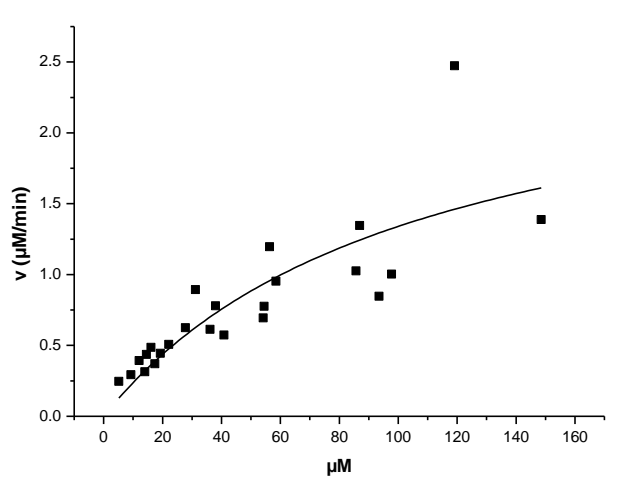

Figure 3.15: Kinetic analyses of PpAOS1 with different hydroperoxides from $\mathrm{C}_{18}$ fatty acids as substrates. The reactions wereperformed in $50 \mathrm{mM}$ sodium phosphate buffer, $\mathrm{pH} 8.0$ at $\mathrm{RT}$ and started by addition of $0.1 \mathrm{nM}$ $(A, B, C, D)$ or $0.05 \mathrm{nM}(E, F)$ PpAOS1. The initial time-dependent changes at $234 \mathrm{~nm}$ were measured. A-F shows Michaelis-Menten diagrams for the conversions of PpAOS1 with A: 9-HPOD, B:13-HPOD, C: 9 $\alpha-H P O T, D: 13 \alpha-$ HPOT,E: $9 \gamma$-HPOT and F: 13y-HPOT.For each data point the substrate concentration was measured again.

Figure 3.16 shows the Michaelis-Menten diagram for the reaction of PpAOS1 with the $C_{20}$ fatty acid derived hydroperoxide 12-HPETE. 


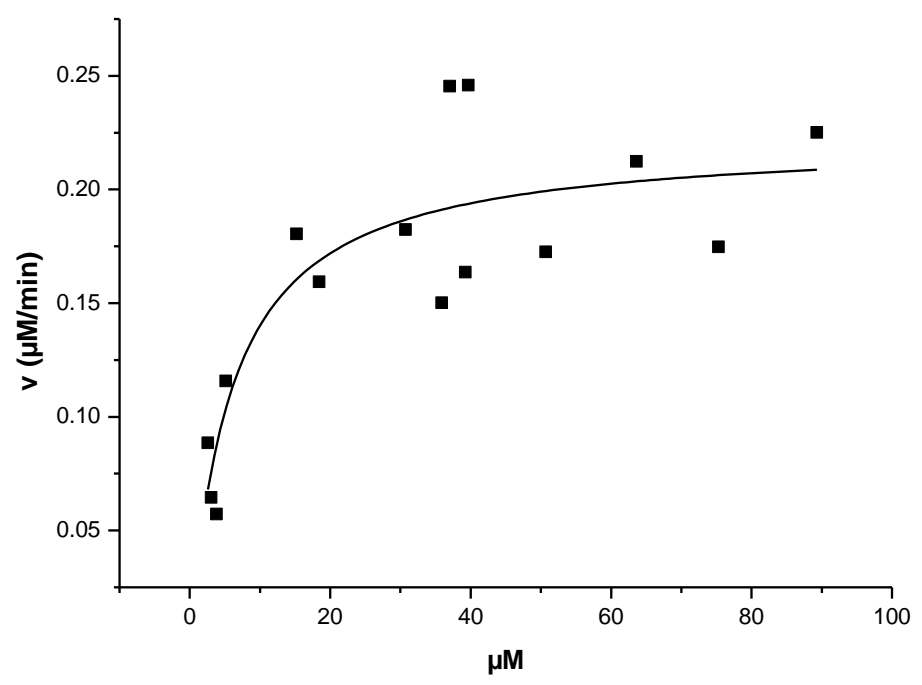

Figure 3.16: Kinetic analysis of PpAOS1 with 12-HPETE. The Michaelis-Menten diagram is shown. The reaction was performed in $50 \mathrm{mM}$ sodium phosphate buffer, $\mathrm{pH} 8.0$ at RT and was started by addition of $0.1 \mathrm{~nm}$ PpAOS1.The initial time-dependent changes at $234 \mathrm{~nm}$ were measured. For each data point the substrate concentration was measured again.

The measured data points were plotted to the Michaelis-Menten equation and the derived parameters are summarized in Table 3.3. The values for $k_{\text {cat }}$ were calculated by considering heme occupancy of approx. $30 \%$. The values for $\mathrm{k}_{\mathrm{cat}} / \mathrm{K}_{\mathrm{M}}$ show that the PpAOS1 isomerized both 9 - and 13hydroperoxides derived from LA, $\alpha$-LeA, and $\gamma$-LeA with a similar specificity as 12-HPETE.

Table 3.3: Kineticparameters for the reaction of PpAOS1 with different hydroperoxides from $\mathrm{C}_{18}$ - and $\mathrm{C}_{20}$ fatty acids. The kinetic parameters were determined by measuring the initial time-dependent substrate consumption at $234 \mathrm{~nm}$ at different substrate concentrations. For analysis between 20 and 30 data points were fitted to the Michaelis-Menten equation. The concentration of PpAOS1 was $0.1 \mathrm{nM}$ except for the measurements of $9 \mathrm{y}$ - and $13 \mathrm{\gamma}$-HPOT $(0.05 \mathrm{nM})$. The values for $\mathrm{k}_{\mathrm{cat}}$ were calculated by considering heme occupancy of approx. $30 \%$.

\begin{tabular}{|c|c|c|c|c|}
\hline substrate & $\begin{array}{c}\mathrm{K}_{\mathrm{M}} \\
{[\mu \mathrm{M}]}\end{array}$ & $\begin{array}{c}\mathrm{V}_{\max } \\
{[\mu \mathrm{M} / \mathrm{min}]}\end{array}$ & $\begin{array}{c}\mathbf{k}_{\mathrm{cat}} \\
{[1 / \mathrm{min}]}\end{array}$ & $\begin{array}{c}k_{\mathrm{cat}} / \mathrm{K}_{\mathrm{M}} \\
{\left[\mathrm{min}^{-1} \mathrm{M}^{-1} \times 10^{6}\right]}\end{array}$ \\
\hline 9-HPOD & $121+/-61$ & $0.98+/-0.29$ & 32680 & 270 \\
\hline $9 \alpha-$ НРОТ & $39+/-14$ & $0.20+/-0.03$ & 6706 & 172 \\
\hline $9 \gamma$-НРОТ & $46+/-17$ & $2.35+/-0.37$ & 156733 & 3426 \\
\hline 13-HPOD & $83+/-42$ & $0.96+/-0.27$ & 31856 & 384 \\
\hline $13 \alpha-\mathrm{HPOT}$ & $95+/-27$ & $0.90+/-0.16$ & 30070 & 316 \\
\hline $13 \gamma-\mathrm{HPOT}$ & $107+/-57$ & $2.77+/-0.88$ & 184500 & 1731 \\
\hline 12-HPETE & $7+/-2$ & $0.23+/-0.02$ & 7657 & 1176 \\
\hline
\end{tabular}




\subsubsection{Site-directed mutagenesis}

To investigate if the site-directed mutagenesis-concept reported before for AtAOS (Lee et al., 2008) can be applied to PpAOS and PpHPL different variants were generated. Determinants were chosen which were essential for the alteration of the enzyme activity as it was shown for AtAOS. Whereas the AtAOS showed specificity for 13-hydroperoxides (Lee et al., 2008), the PpAOS1 and PpHPL were unspecific enzymes.

\begin{tabular}{|c|c|}
\hline AtAOS & DKVEKKDLETGTYMPSTELTGGYRIISYYLDPSEPK \\
\hline PaAOS & SKVEKKDLETGTYMPSTKLTGAYRVISYLDPSEPR \\
\hline LeAOS3 & SQVDKENYFEGTFMSSPSFNGGYKY \\
\hline PpAOS & KVEKKDVETGTYMPSVSFTSGY \\
\hline PpAOS2 & NKCEKRDLFLGTYMPDLSYTSGHRVI \\
\hline AtHPL & DLVDKRDVIIGDFRPSLGFYGGVRVGVYLDTTEPK \\
\hline LeHPL & EIVEKANVIVGDFMPSVVYTGDMRVDAYLDTSEPK \\
\hline PpHPL & DKVDKYKAEAGTIMPSTEYNGGYEVDAYLDASDKK \\
\hline StDES & GTFKPGKEYYSGYRPVAFIDTKDPN \\
\hline AsDES & SKVSKVNSLTGNYMPALSETGGYRV \\
\hline BfEAS & FGVA-EVRRDFTEGI \\
\hline BfEAS_1 & IDLFKKEEFCFGVVGEVRKDFTEGVC \\
\hline BfEAS_2 & VDLFRKEEEYFGPI-EVRKDFTEGVCPSITSNGKI \\
\hline
\end{tabular}

Figure 3.17: Partial multiple sequence alignment of different CYP74-enzymes from different plant species and marine invertebrates. Allium sativum (As); Arabidopsis thaliana (At); Branchiostoma floridae (Bf); Lycopersicum esculentum (Le); Parthenium argentatum (Pa); Physcomitrella patens (Pp); Solanum tuberosum (St); AtAOS, CAA63266; AsDES, AJ867809; AtHPL, AAC69871; BfEAS, EU555186; BfEAS_1, XP2601390; BfEAS_2, XP2602690; LeAOS3, AAN76867; LeHPL, AAF67142; PaAOS, CAA55025; PpAOS1,CAC86919; PpAOS2, XP_001759629; PpHPL, CAC86920; StDES, CAC28152. The highly conserved residues are in grey brackets. Amino acid positions used for site-directed mutagenesis in this study are marked in bold letters.

Sequence alignments in Figure 3.17 showed that particular amino acid-residues which have been shown to be important for the product specificity in AtAOS are also conserved in PpAOS1 and PpAOS2. Thus both AOS isozymes contain a strictly conserved phenylalanine $(F)$ at position 93 which is an essential residue for the AOS activity (Lee et al., 2008). It is suggested that this F-residue stabilizes an intermediately formed carbon-centered substrate radical. The essential residue at this position in HPL is a leucine (L), which cannot stabilize reactive product intermediates and leads therefore to the formation of an unstable hemiacetal, which decomposes spontaneously to short chain aldehydes (Grechkin and Hamberg, 2004).

Interestingly, sequence alignments showed, that in PpHPL an AOS-specific F-residue instead of that for HPL characteristic $L$ can be found at this particular position (F151; PpHPL numbering). To analyze the product activity and the importance of this residue the $F$ was substituted by L (PpHPL_F151L) (Lee et al., 2008).Additionally the PpAOS1_F93L variant was generated.

At position 111 a conserved AOS-specific serine $(S)$ in PpAOS1 is located, which was substituted by an HPL-specific alanine (A) (PpAOS1_S111A). Additionally in HPL the PpHPL_A169S variant was generated (Lee et al., 2008). 


\begin{tabular}{lll}
\hline AtAOS & REEATHNLLFATCFNTWGGMKILFPNMV-KRIGRA 340 \\
PaAOS & KDEAVHNILFAVCFNTFGGVKILFPNTL-KWIGVA 295 \\
LeAOS3 & RDEACHNFVFLAGFNSYGGLKVFFPSLI-KWIGTS 315 \\
PpAOS1 & RNDATANLLFFLCFNAYGGFSIFFPLIT-ILISSC 300 \\
PpAOS2 & RDDAIANLLFLLGFNAYGGFNFFFPQT-GHIAQC 299 \\
AtHPL & RDEAIQNLLFVLGFNAYGGFSVFLPSLIGRITGD- 216 \\
LeHPL & EQEAIHNLLFILGFNAFGGFSIFLPTLLGNLGDEK 207 \\
PpHPL & QDEAVHNLIFFLILNAHGGFCRFLPVIL-REVAKN 356 \\
StDES & REEAVQNILFLVGINMFAGLNAFSPHLF-RFVGEA 301 \\
AsDES & REEAIHHLIFTWAINAYLGIRTCLMRLF-KWIVAS 295 \\
BfEAS & EAETTAQLLLPVFFNGVAGIRANLVSSF-ARLDTI 243 \\
BfEAS_1 & EKEATAQLLFPVFINGAYGLAAHLVCTF-ACLDTI 244 \\
BfEAS_2 & EKETTGQLLFSIMENGVGGMAVNLVPSF-AHLDTI 366
\end{tabular}

Figure 3.18: Partial multiple sequence alignment of different CYP74-enzymes from different plant species and marine invertebrates. Allium sativum (As); Arabidopsis thaliana (At); Branchiostoma floridae (Bf); Lycopersicum esculentum (Le); Parthenium argentatum (Pa); Physcomitrella patens (Pp); Solanum tuberosum (St); AtAOS, CAA63266; AsDES, AJ867809; AtHPL, AAC69871; BfEAS, EU555186; BfEAS_1, XP2601390; BfEAS_2, XP2602690; LeAOS3, AAN76867; LeHPL, AAF67142; PaAOS, CAA55025; PpAOS1,CAC86919;PpAOS2, XP_001759629; PpHPL, CAC86920; StDES, CAC28152. The highly conserved residues are in grey brackets. Amino acid positions used for site-directed mutagenesis in this study are marked in bold letters.

For tomato AOS was reported that there is a further F (LeAOS3_F295) which is strictly conserved in AOS and HPL (Toporkova et al., 2008). In PpHPL is at this position an AOS-specific F instead of that HPL-specific L (Figure 3.18). Therefore the PpHPL_L336F variant was generated. For LeAOS3 was also reported that the variant with substitution of lysine (K) by S (LeAOS3_K302S) has AOS- and HPLactivities (Toporkova et al., 2010). In PpAOS1 S is at this position and in PpHPL cysteine (C). In both enzymes the residues were substituted by K (PpAOS1_S287K and PpHPL_C343K).

Site-directed mutagenesis of strictly conserved asparagine $(N)$ by a glutamine (Q) in AtAOS (AtAOS_N321Q) showed abolish enzyme activity (Lee et al., 2008). In PpAOS1 and PpHPL these residues were substituted (PpAOS1_N281Q and PpHPL_N337Q).

\subsubsection{Spectroscopic analysis}

\subsection{UV/vis analysis}

In order to investigate if the site-directed mutations effect protein folding and cofactor incubation, the purified variants were analyzed via UV/vis and CD spectroscopy (2.3.15).

In Figure 3.19 the UV/vis spectra of PpHPL variants F151L (A), A169S (B), and F151L, A169S (C) are shown. The spectra of the various PpHPL variants were similar to the spectrum of the wild type (Figure 3.19 D). PpHPL_F151L (Figure 3.19 A) had absorption maxima at $415 \mathrm{~nm}$ (Soret-band), $570 \mathrm{~nm}$ ( $\alpha$-band), $540 \mathrm{~nm}(\beta-), 280 \mathrm{~nm}$ ( $\gamma$-band), and $350 \mathrm{~nm}$ ( $\delta$-band). The maxima of PpHPL_A169S (Figure $3.19 \mathrm{~B}$ ) were at $414 \mathrm{~nm}$ (Soret-band), $570 \mathrm{~nm}$ ( $\alpha$-band), $531 \mathrm{~nm}(\beta-), 278 \mathrm{~nm}(\gamma$-band), and $350 \mathrm{~nm}(\delta-$ band). The Soret-band of the PpHPL double-mutant_F151L, A169S (Figure $3.19 \mathrm{C}$ ) was shifted to an absorption maximum at $421 \mathrm{~nm}$, the $\alpha$-band was at $570 \mathrm{~nm}, \beta$-band at $540 \mathrm{~nm}, \gamma$-band at279 nm, and the $\delta$-band at $357 \mathrm{~nm}$. 
A

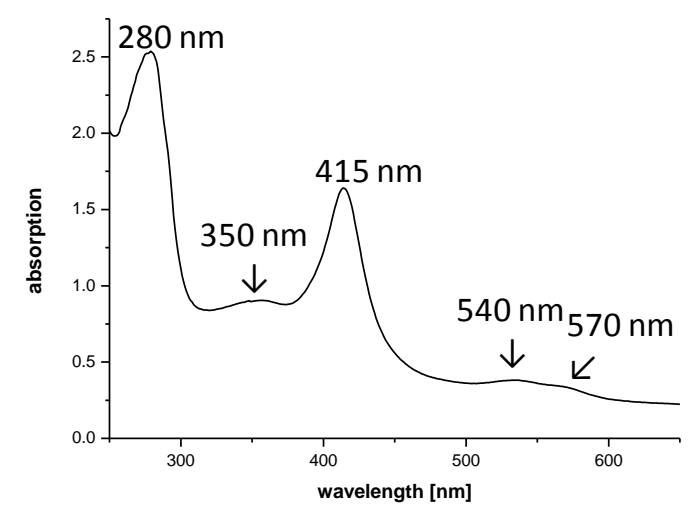

C

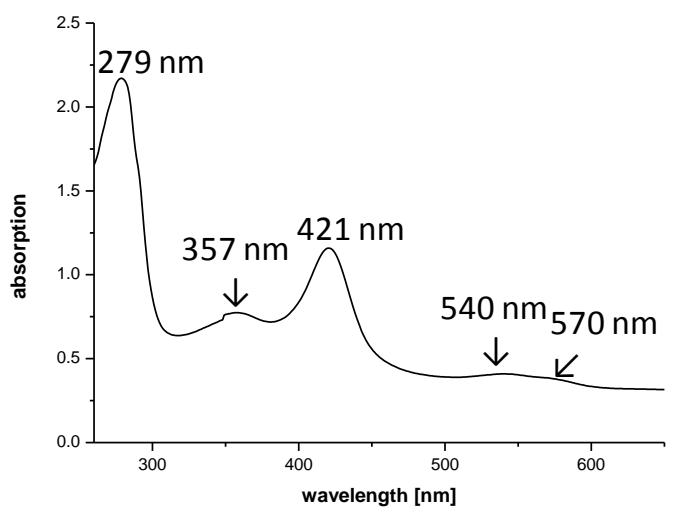

B

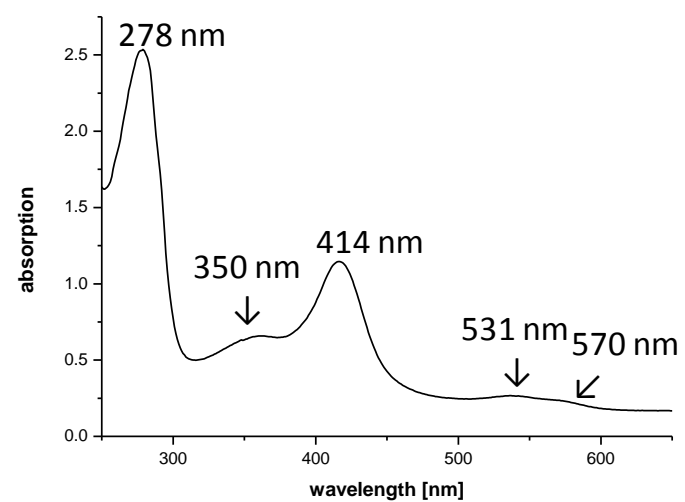

D

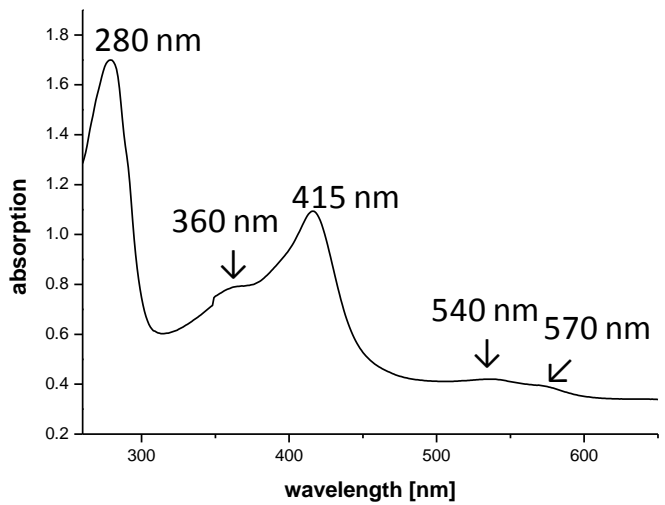

Figure 3.19: UV/vis spectra of purified PpHPL_F151L (A), PpHPL_A169S (B), and PpHPL_F151L, A169S (C) in comparison to the wild type (D). They were measured in $50 \mathrm{mM}$ sodium phosphate buffer, $\mathrm{pH}$ 8.0.The spectra are representative for one to three experiments.

The calculated heme content for the wild type was of $46 \%$. The heme content of PpHPL_F151L is of about $45 \%$ and therefore like the content of wild type. Whereas the heme content of the two other variants is slightly lower. PpHPL_A169S has a heme content of $31 \%$ and the double variant PpHPL_F151L, A169S of $37 \%$.

\subsection{CD spectroscopy}

For the analysis of the native protein folding of the variants $C D$ spectra were measured in a range of $260 \mathrm{~nm}$ to $190 \mathrm{~nm}$ (Figure 3.20). The spectra of PpHPL_A169S (A) and PpHPL_F151L, A169S (B) were similar to that of the wild type (C). Both variants had also the negative peaks at $215 \mathrm{~nm}$ and $220 \mathrm{~nm}$ and the positive peak at $193 \mathrm{~nm}$ which are typical for proteins consisting of $\alpha$-helix (Panagakou et al., 2012). 
A

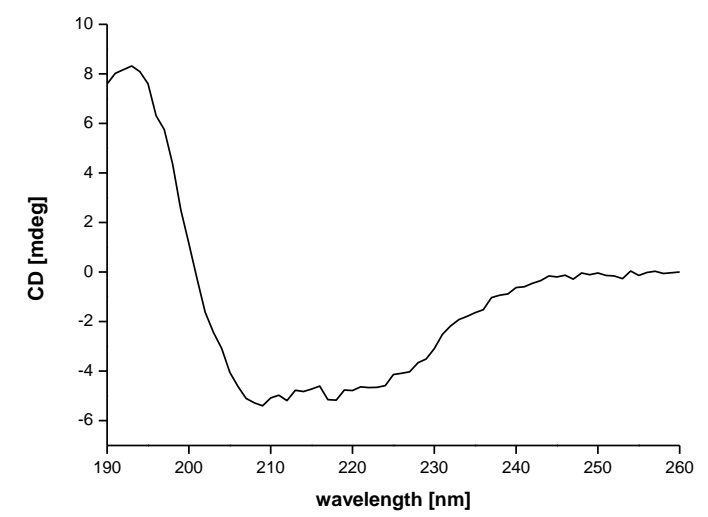

C

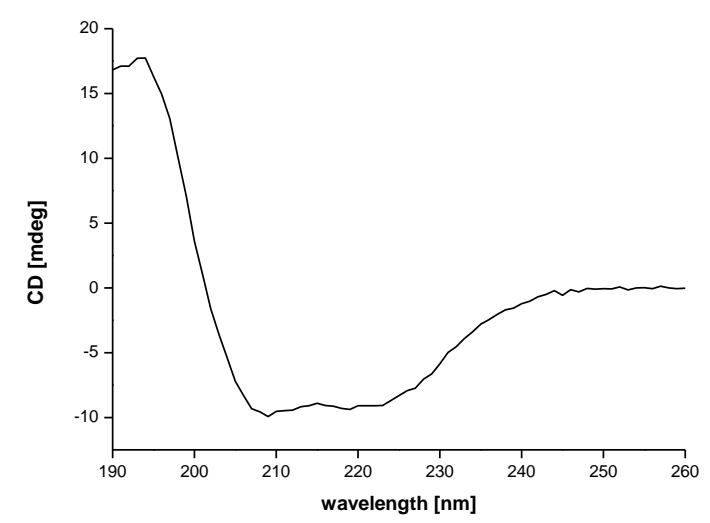

B

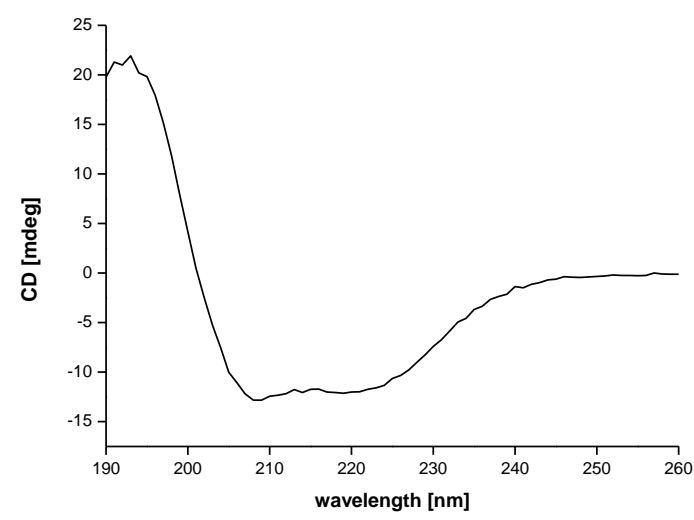

Figure 3.20: $C D$ sepcra of purified variants from PpHPL in comparison to the wild type. They were measured in $50 \mathrm{mM}$ sodium phosphate buffer, $\mathrm{pH} 8.0$ on a Chirascan ${ }^{\mathrm{TM}}$ CD Spectrometer (Applied Photophysics Ltd., Leatherhead, United Kingdom). The spectra were recorded in the range of $260 \mathrm{~nm}$ to $190 \mathrm{~nm}$. A: PpHPL_A169S, B: PpHPL_F151L, A169S, and C: PpHPL wild type.The figure shows the spectra of oneexperiment.

Therefore these measurements showed that the single amino acid exchanges had no impact on the folding of the proteins.

\subsubsection{Analysis of products}

\subsection{Radio-HPLC}

Spectroscopic analysis showed that site-directed mutagenesis had no effect on protein folding. To analyze if the substitutions of some amino acid residues can alter enzymeactivity product analysis were done. The products of the conversions of PpAOS1 variants and PpHPL variants with radiolabeled 9- and 13-hydroperoxides were analyzed by radio-HPLC. The conversions were performed as described in 2.3.13.2.

In Figure 3.21 the product analysis for the variant PpAOS1_F93L with 9-HPOD is exemplarily shown. For comparison the analysis of incubations with PpAOS1 wild type and PpHPL wild type are also shown in this chromatogram. Incubations with the PpAOS1_F93L variant led to a strong decrease in the classical AOS product, the $\alpha$-ketol-derivative (RT $7.5 \mathrm{~min}$ ), whereas an increase of the HPLproduct 
9-oxo nonanoic acid (RT 2 min) occurred. Incubations with 9 $\alpha$-HPOT showed similar product formations for the AOS variant (see appendix Figure 9.2A).

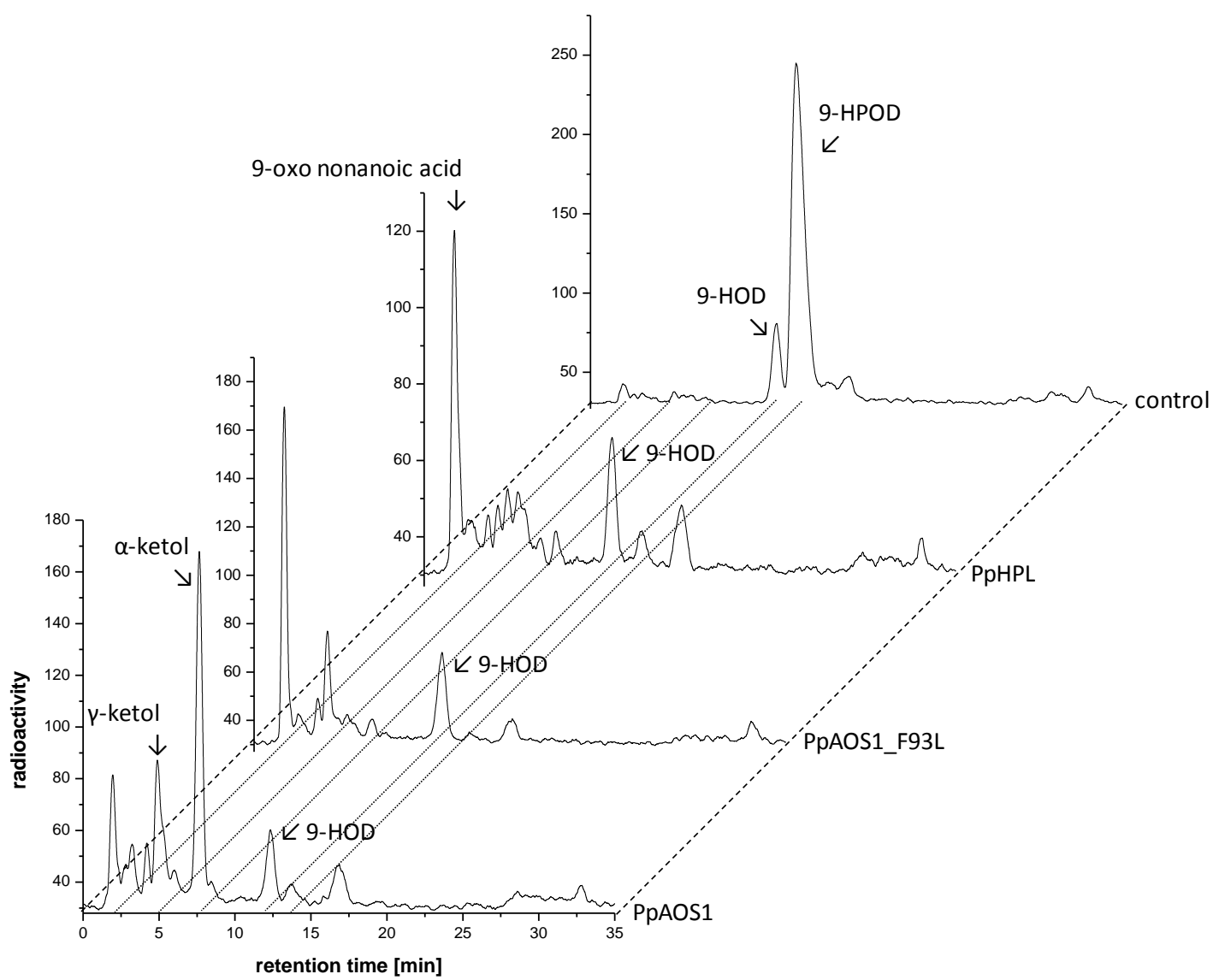

Figure 3.21: Product analysis of PpAOS_F93L with $\left[1-{ }^{14} \mathrm{C}\right]-9-\mathrm{HPOD}$ in comparison to the PpAOS1 and PpHPL wild types. The purified enzymes as well as only buffer (control) were incubated with the radio-labeled substrate for $30 \mathrm{~min}$ at RT. After extraction with diethyl ether the products were analyzed by RP-HPLC coupled to a radiodetector.The chromatograms shown are representative for two to three experiments.

The incubation with $13 \alpha$-HPOT showed also a strong decrease in the AOSproduct, $\alpha$-ketol-derivative (RT $5.5 \mathrm{~min}$ ), and a light increase of the HPLproduct (RT $2.5 \mathrm{~min}$ ) (Figure 3.22). 13-HPOD incubations gave similar results (see appendix Figure 9.2 B). 


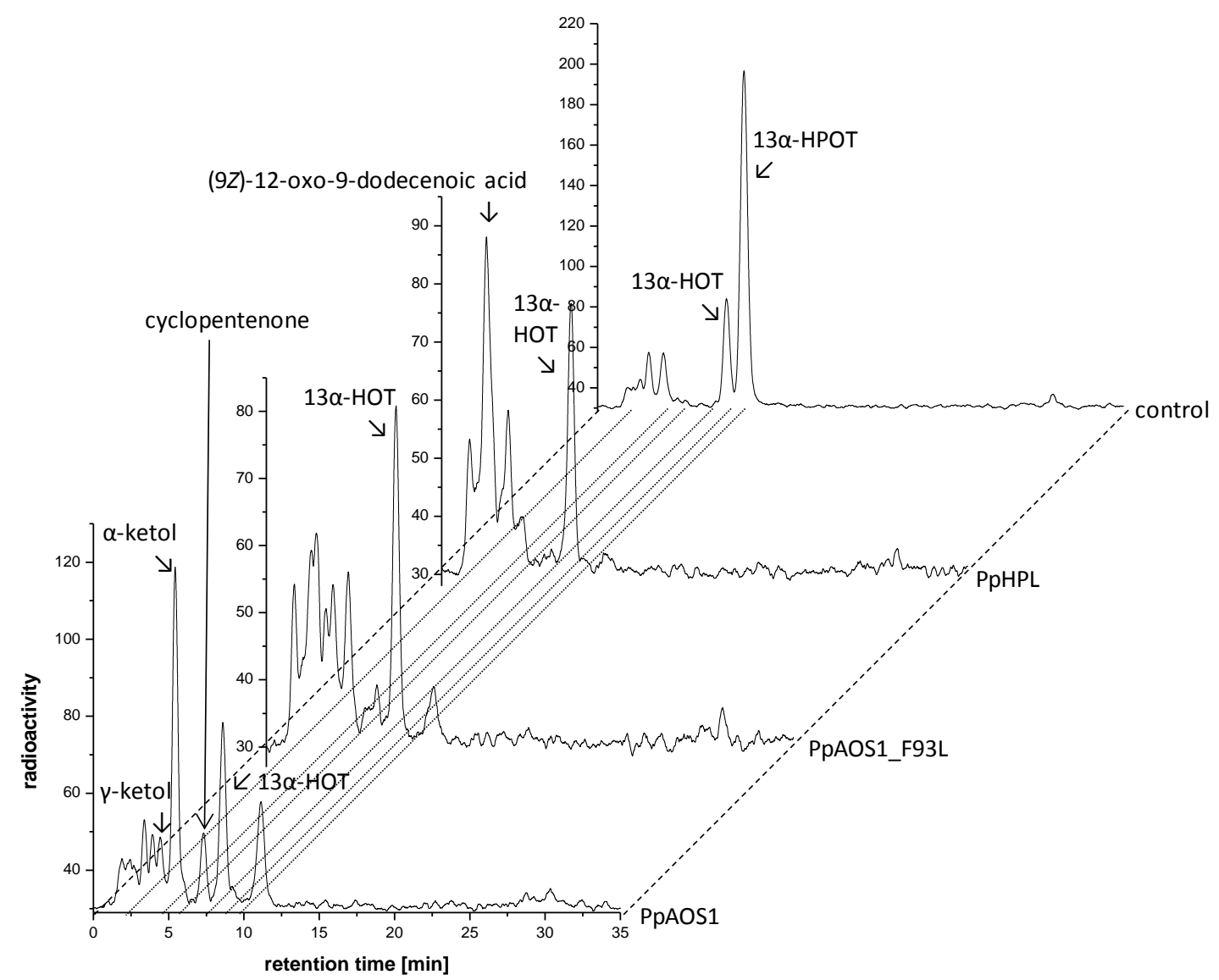

Figure 3.22: Product analysis of PpAOS_F93L with $\left[1-{ }^{14} \mathrm{C}\right]-13 \alpha-\mathrm{HPOT}$ in comparison to the PpAOS1 and PpHPL wild types. The purified enzymes as well as only buffer (control) were incubated with the radio-labeled substrate for $30 \mathrm{~min}$ at RT. After extraction with diethyl ether the products were analyzed by RP-HPLC coupled to a radio-detector.The chromatograms shown are representative for two to five experiments.

It seems that the $\mathrm{F}$ at position 93 (PpAOS1_F93) is an essential determinant for the dehydration of both 9- and 13-hydroperoxides. Therefore an appropriate variant for PpHPL was generated (PpHPL_F151L). The product analysis of PpHPL_F151L for all 9 $\alpha-$, 13- and 13 $\alpha$-hydroperoxides showed no changes in its product formations and was similar to that of wild type. The generated PpHPL_A169S variant in which a conserved HPL-specific alanine (A) was substituted by a AOS-specific serine (S), which has been reported to determine AOS activity, also did not showed any alterations in its product formations. As well the double variant PpHPL_F151L/A169S showed no changes in its specificity. The results for the PpHPL variants and the PpAOS1_F93L variant compared to the wild type enzymes are summarized in Table 3.4. 
Table 3.4: Product specificities of different PpHPL and PpAOS1 variants. Affinity purified enzymes were incubated with $\left[1-{ }^{14} \mathrm{C}\right]$-labeled hydroperoxy fatty acids for approx. $30 \mathrm{~min}$ at RT. After extraction with diethyl ether the isolated products were analyzed by RP-HPLC that was coupled to a radio-detector and quantified by integration of the respective peak area. The relative amounts of each product is indicated by the number of "+".

\begin{tabular}{|c|c|c|c|c|}
\hline substrate & enzyme-variant & $\begin{array}{c}\text { w-oxo fatty acids } \\
\text { (=HPL activity) }\end{array}$ & $\begin{array}{c}\text { ketols } \\
\text { (= AOS activity) }\end{array}$ & $\begin{array}{l}\text { cyclopentenone } \\
\text { (= AO cyclization) }\end{array}$ \\
\hline \multirow[t]{3}{*}{ 9-HPOD } & HPL & ++++ & + & n. d. \\
\hline & AOS1 & + & ++++ & n. d. \\
\hline & AOS1_F93L & ++++ & + & n. d. \\
\hline \multirow[t]{6}{*}{$9 \alpha-H Р O T$} & HPL & ++++ & + & n. d. \\
\hline & HPL_F151L & ++++ & + & n. d. \\
\hline & HPL_A169S & ++++ & + & n. d. \\
\hline & HPL_F151L/A169S & ++++ & + & n. d. \\
\hline & AOS1 & + & ++++ & n. d. \\
\hline & AOS1_F93L & ++++ & - & n. d. \\
\hline \multirow[t]{6}{*}{ 13-HPOD } & $\mathrm{HPL}$ & ++++ & + & n. d. \\
\hline & HPL_F151L & ++++ & + & n. d. \\
\hline & HPL_A169S & ++++ & + & n. d. \\
\hline & HPL_F151L/A169S & ++++ & + & n. d. \\
\hline & AOS1 & + & ++++ & n. d. \\
\hline & AOS1_F93L & ++++ & + & n. d. \\
\hline \multirow[t]{6}{*}{$13 \alpha-H P O T$} & $\mathrm{HPL}$ & ++++ & + & n. d. \\
\hline & HPL_F151L & ++++ & + & n. d. \\
\hline & HPL_A169S & ++++ & + & n. d. \\
\hline & HPL_F151L/A169S & ++++ & + & n. d. \\
\hline & AOS1 & + & +++ & + \\
\hline & AOS1_F93L & ++ & ++ & + \\
\hline
\end{tabular}




\subsection{Biochemical characterization of BfEAS}

For comparison of catalysis of the different CYP74-enzymes BfEAS from the lancelet B. floridae was characterized.

\subsubsection{Cloning and expression}

Codon optimized DNA encoding for the amino acid sequence of BfEAS with a C-terminal hexahistidine peptide and an additional amino acid sequence (MAKKTSS) at the N-terminus was synthesized.The amino acids transition between the C-terminal His-tag and the protein is DLEHHHHHH and between the additional amino acid sequence at the $\mathrm{N}$-terminus and the protein is MAKKTSSM.

Determination of optimal expression conditions was done in E. coli BL21 Star cells. Therefore various expression conditions after the induction with $0.1 \mathrm{M}$ IPTG were tested. BfEAS was either incubated at $11{ }^{\circ} \mathrm{C}$ for $1 \mathrm{~d}$, at $16^{\circ} \mathrm{C}$ for $3 \mathrm{~d}$ or at $28^{\circ} \mathrm{C}$ for $1 \mathrm{~d}$. In order to improve EAS expression $\delta$-amino levulinoic acid and ammonium ferric citrate as cofactors was added to the cultures.

In the following figures the SDS-PAGE analyses of purifications of BfEAS expressed in E. coli BL21 Star that were grown under the different expression conditions were shown. The SDS-PAGE analyses shown in Figure 3.23 illustrates that the cultivations for $1 \mathrm{~d}$ at $11^{\circ} \mathrm{C}(\mathrm{A})$ and for $1 \mathrm{~d}$ at $28^{\circ} \mathrm{C}(\mathrm{B})$ led to the production of a similar protein amount.

A

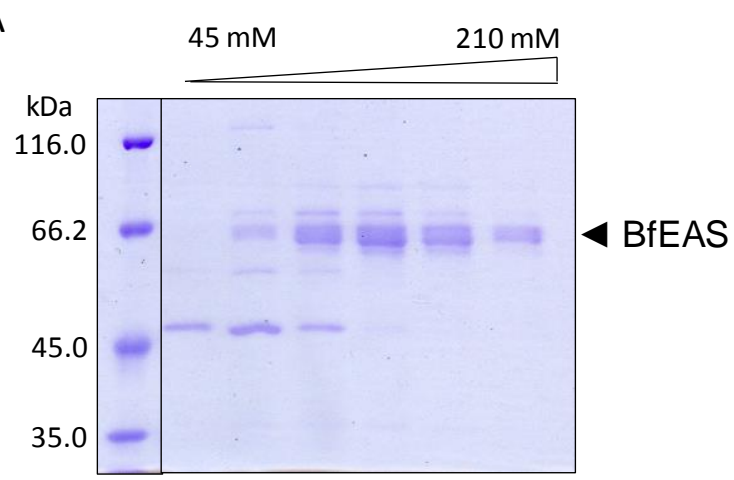

B

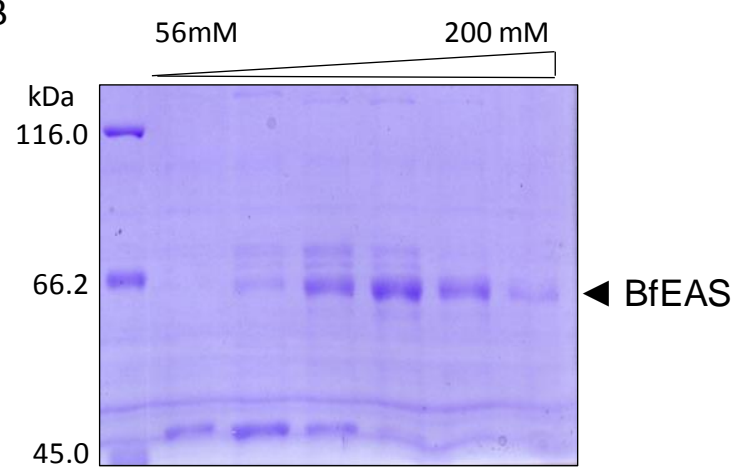

Figure 3.23: SDS-PAGE analyses of BfEASpurified from different E. coli BL21 Star cultivations $\left(11^{\circ} \mathrm{C}\right.$ or at $28^{\circ} \mathrm{C}$ for $1 \mathrm{~d}$ ). They were cultivated either at $11^{\circ} \mathrm{C}$ for $1 \mathrm{~d}$ (A, $10 \%$ gel) or at $28^{\circ} \mathrm{C}$ for $1 \mathrm{~d}(\mathrm{~B}, 8 \%$ gel).Cells were harvested and disrupted by sonification. The cell debris was removed by centrifugation. BfEAS was purified by $\mathrm{Ni}^{2+}$-affinity chromatography and eluted from the column by a linear gradient with an increasing imidazole concentration (15 mM to $300 \mathrm{mM}$ ). The figure shows the SDS-PAGEsof one purification.

The most BfEAS protein was obtained from E. coli BL21 Star that was cultivated at $16^{\circ} \mathrm{C}$ for $3 \mathrm{~d}$ as shown in the SDS-PAGE in Figure 3.24. These optimized conditions were used for the further expressions. 


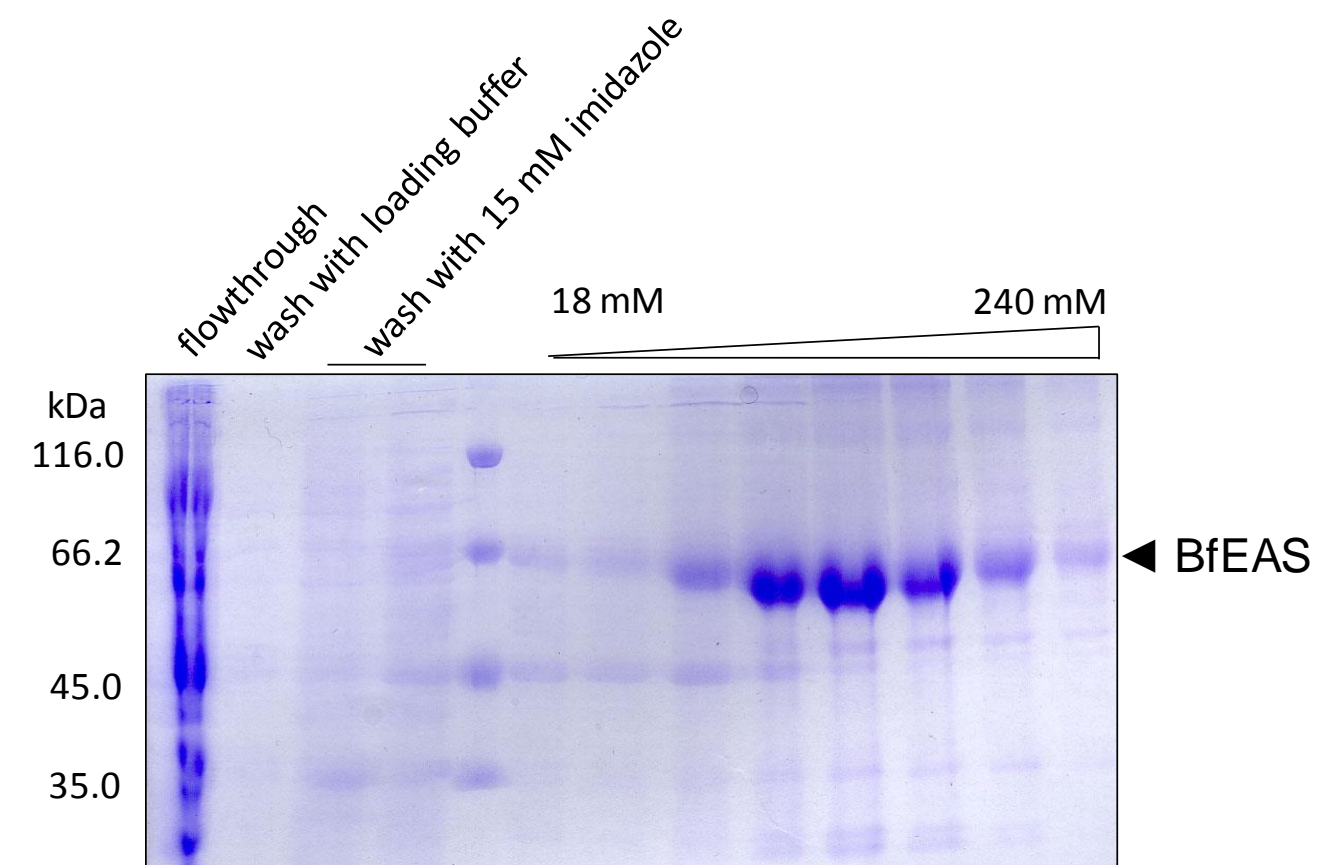

Figure 3.24: SDS-PAGE of purified BfEAS expression at $16^{\circ} \mathrm{C}$ for $3 \mathrm{~d}$. The enzyme was heterologously expressed in BL21 Star. The cells were harvested and disrupted by sonification. The cell debris was removed by centrifugation. The protein was purified by $\mathrm{Ni}^{2+}$-affinity chromatography and eluted from the column by a linear gradient with an increasing imidazole concentration (15 mM to $300 \mathrm{mM}$ ). The figure is representative for at least five purifications.

\subsubsection{Purification}

BfEAS was purified by an optimized purification protocol that was established in this work (2.3.8.1): After membrane preparation (see 2.3.7) the resulting supernatant was loaded onto a $1 \mathrm{ml} \mathrm{His-trap}$ column. Unspecifically bound proteins were washed from the column with $15 \mathrm{mM}$ imidazole. Subsequently, bound BfEAS was eluted with a gradually increasing imidazole concentration of $15 \mathrm{mM}$ to $300 \mathrm{mM}$. Activity tests (2.3.11.1) and SDS-PAGE analysis (2.3.9) were done with collected fractions. SDS-PAGE analyses of purifications of BfEAS that was isolated from E. coli BL21 Star grown under different expression conditions as shown in Figure 3.23 and Figure 3.24 illustrate clear protein bands at $66 \mathrm{kDa}$ for eluted BfEAS. Very high amounts of homogenous protein (approx. $35 \mathrm{mg}$ protein/ml expression cultures) were obtained and used for further analysis.

By size exclusion chromatography a native molecular weight of $60 \mathrm{kDa}$ was determined for BfEAS (data not shown). SDS-PAGE showed a molecular weight of approx. $60 \mathrm{kDa}$ and theoretical calculated mass was $66 \mathrm{kDa}$. The molecular mass determined by size exclusion chromatography is thus consistent with the theoretical mass of $66 \mathrm{kDa}$ indicating that BfEAS exists as a monomeric protein. 


\subsubsection{Spectroscopic analysis}

\subsubsection{UV/vis analysis}

Purified BfEAS showed a deep reddish color, which indicated the presence of a heme cofactor. Therefore UV/vis spectra were measured and the heme content was determined. The spectrum of BfEAS showed an absorption maximum of the Soret-band at $420 \mathrm{~nm}$, whereas the maxima of $\alpha$-band was at $565 \mathrm{~nm}, \beta$-band at $539 \mathrm{~nm}, \gamma$-band at $281 \mathrm{~nm}$, and $\delta$-band at $368 \mathrm{~nm}$ (Figure 3.25).

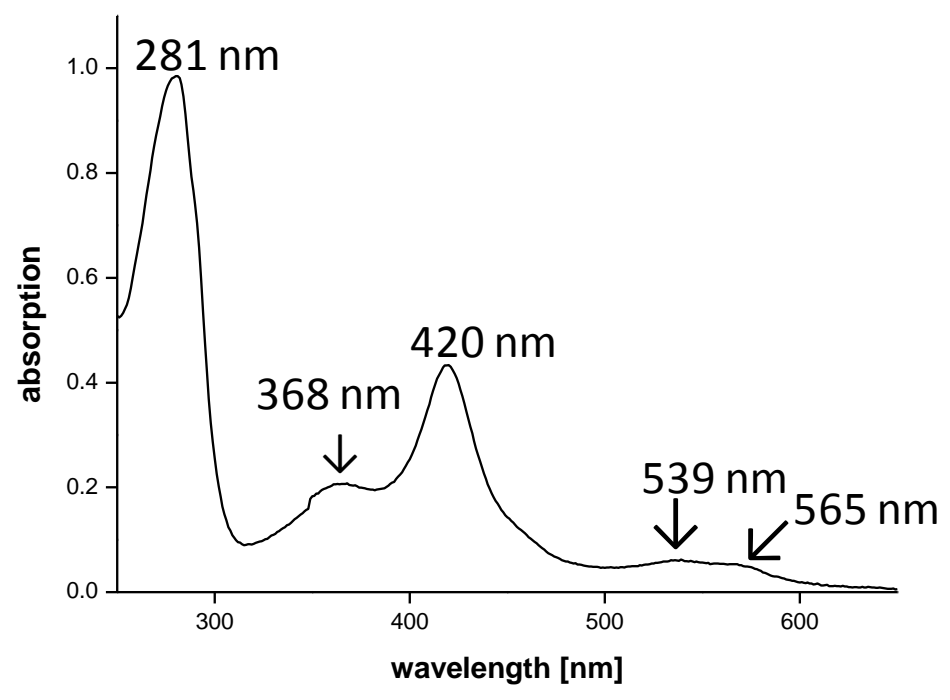

Figure 3.25: UV/vis spectrum of purified BfEAS in $50 \mathrm{mM}$ sodium phosphate buffer, $\mathrm{pH}$ 8.0. The UV/vis spectrum shows absorption maxima at $281 \mathrm{~nm}(\nu)$ and $420 \mathrm{~nm}$ (Soret) and smaller maxima at $36 \mathrm{~nm}(\delta)$, $539 \mathrm{~nm}(\beta)$, and $565 \mathrm{~nm}(\alpha)$.The figure is representative for three experiments.

The calculated heme content of BfEAS with a theoretical molar extinction coefficient $\varepsilon_{280}(\mathrm{BfEAS}) \approx$ $89.500 \mathrm{M}^{-1} \mathrm{~cm}^{-1}$ and an excepted molar extinction coefficient for the Soret-band of $\varepsilon_{\text {soret }} \approx 100.000 \mathrm{M}^{-}$ ${ }^{1} \mathrm{~cm}^{-1}$, is of approx. $37 \%$.

The BfEAS does not show the characteristic high-spin iron-III-complex signal at $630 \mathrm{~nm}$, suggesting the presence of a mainly low-spin heme-iron complex. 


\subsubsection{CD spectroscopy}

The CD spectrum of BfEAS was measured in $50 \mathrm{mM}$ sodium phosphate buffer, $\mathrm{pH} 8.0$ in a range of $260 \mathrm{~nm}$ to $190 \mathrm{~nm}$ (Figure 3.26).

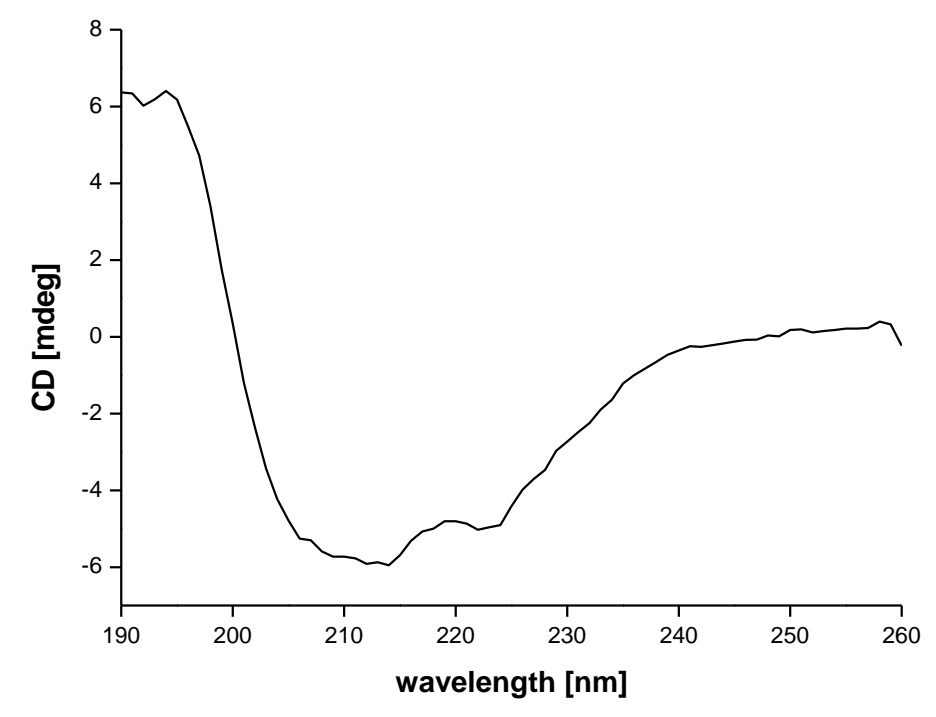

Figure 3.26: CD sepctrum of purified BfEAS. It was measured in $50 \mathrm{mM}$ sodium phosphate buffer, $\mathrm{pH} 8.0$ on a Chirascan $^{\text {TM }}$ CD Spectrometer (Applied Photophysics Ltd., Leatherhead, United Kingdom). The spectrum was recorded in the range of $260 \mathrm{~nm}$ to $190 \mathrm{~nm}$. The figure shows the spectrum of oneexperiment.

The spectrum showed negative peaks at $212 \mathrm{~nm}$ and $222 \mathrm{~nm}$, and a positive peak at $194 \mathrm{~nm}$, which are typical peaks for proteins with a mainly $\alpha$-helical fold (Panagakou et al., 2012).

\subsubsection{Analysis of products}

B. floridae contains the $\omega-3$ fatty acid docosahexaenoic acid. Because of its 6 double bonds (Figure 3.27) upon oxidation formation of twelve different positional isomers is possible of which ten of them may be of enzymatic origin.

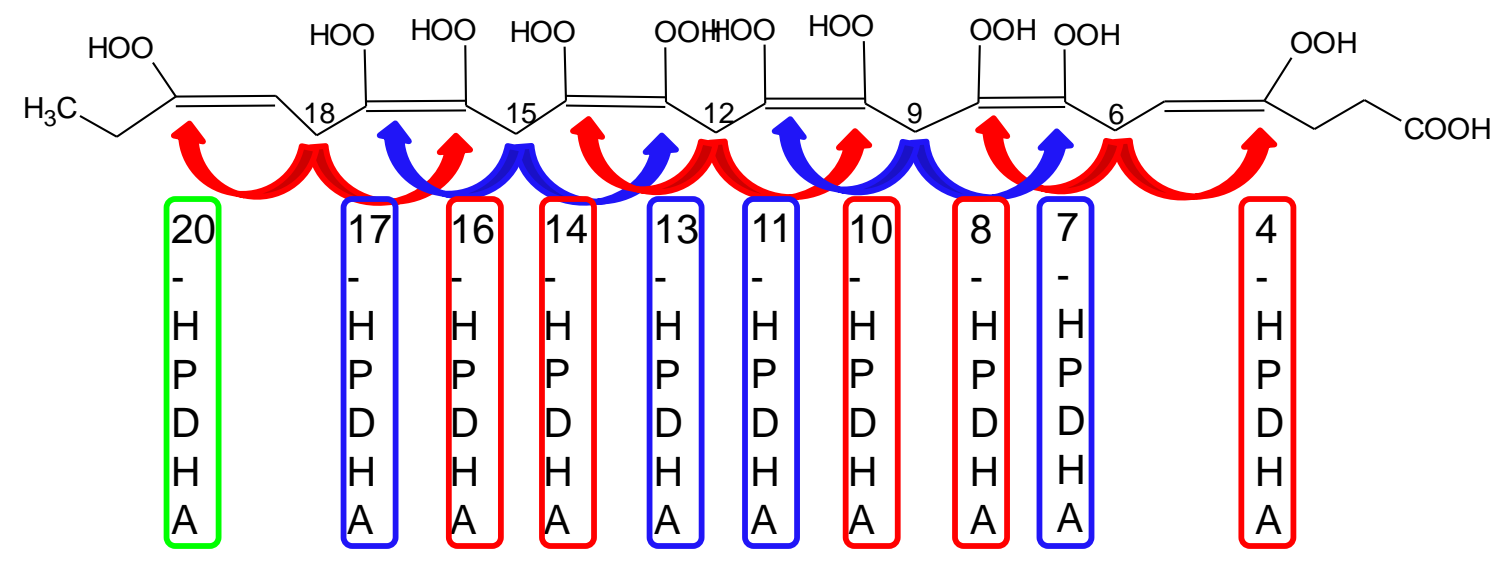

Figure 3.27: Structure of $\omega$-3 fatty acid docosahexaenoic acid (DHA) and its possible ten positions that may be oxidized enzymes. Abbreviations: HPDHA: Hydroperoxy docosahexaenoic acid. 
To determine which products were formed by the conversions of BfEAS with hydroperoxides derived from LA and $\alpha$-LeA as well as with hydroperoxides derived from DHA product analysis of the different reactions were performed.

\subsubsection{Radio-HPLC}

Products formed from incubations of purified BfEAS with radio-labeled 9- and 13-hydroperoxides as substrates were analyzed by RP-HPLC coupled to a radio-detector (2.3.13.2).

In Figure 3.28 the RP-HPLC analysis of products formed from incubation of BfEAS with $\left[{ }^{14} \mathrm{C}\right]-9 \alpha-\mathrm{HPOT}$ as substrate is shown. Besides the main product that eluted after $3.5 \mathrm{~min}$ additional products were detected. Based on the respective RT and absorption maxima, these products were identified as 9oxo nonanoic acid (RT $2 \mathrm{~min}$ ), an epoxy alcohol derivative (RT $3.5 \mathrm{~min}$ ) as well as colnelenic acid (RT $28 \mathrm{~min}$ ). Thus, beside the EAS specific epoxy alcohol product also small amounts of the characteristic HPL product (9-oxo nonanoic acid) as well as a characteristic DES product (colnelenic acid) are formed during catalysis. The peak at about 6 min could not be identified.

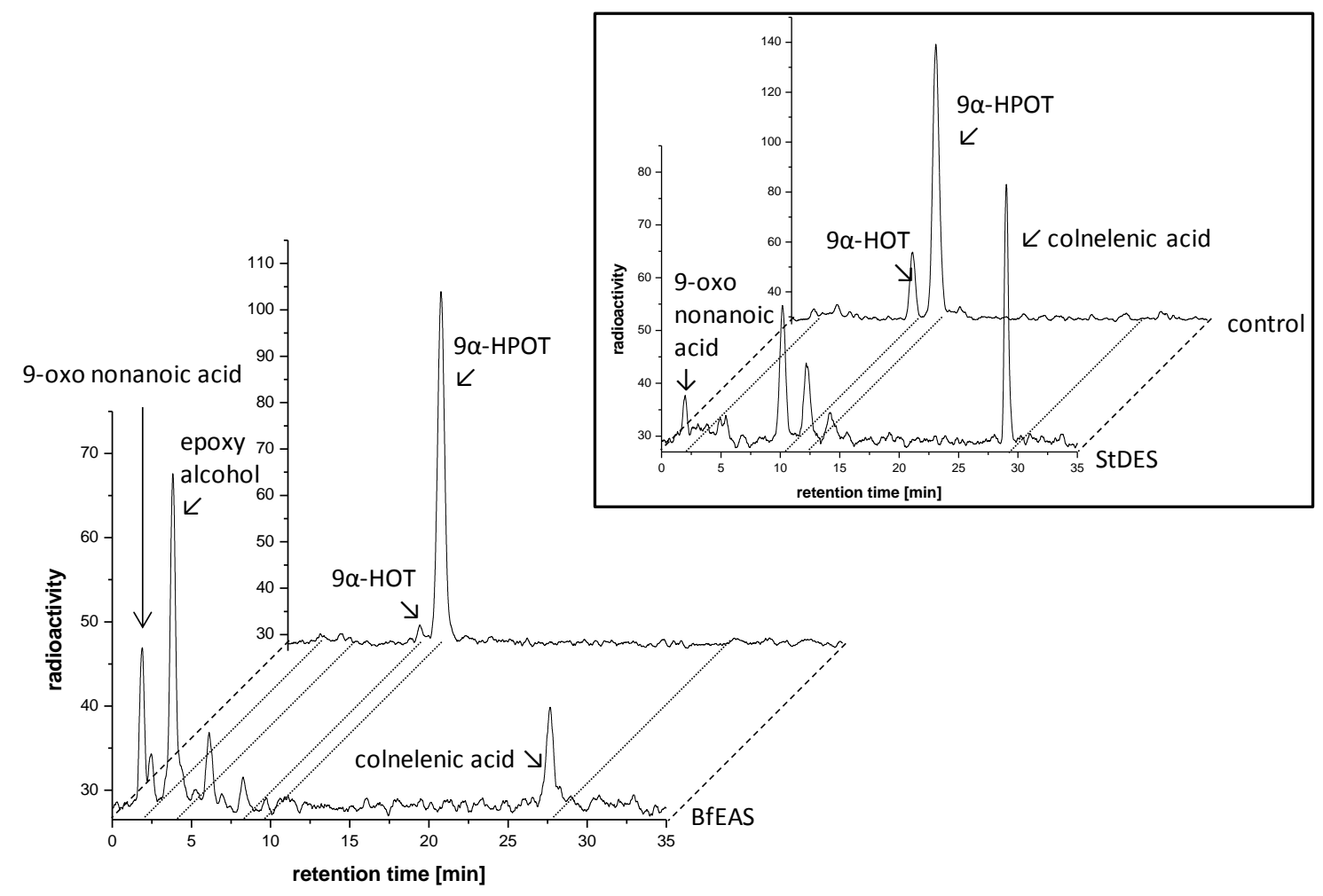

Figure 3.28: Product analysis from incubation of BfEAS with $\left[1-{ }^{14} \mathrm{C}\right]-9 \alpha$-HPOT. The purified enzyme was incubated with the radio-labeled substrate for $30 \mathrm{~min}$ at RT. After extraction with diethyl ether the products were analyzed by RP-HPLC coupled to a radio-detector. The insertion shows product analysis of purified StDES with $\left[1-{ }^{14} \mathrm{C}\right]-9 \alpha$-HРOT.The figure is representative for five experiments. 
The conversion of $\left[{ }^{14} \mathrm{C}\right]-9-\mathrm{HPOD}$ with BfEAS gave a similar product pattern as with $\left[{ }^{14} \mathrm{C}\right]-9 \alpha-\mathrm{HPOT}$ as substrate: an epoxy alcohol derivative, 9-oxo nonanoic acid as well as colneleic acid could be detected (see appendix Figure 9.3 A).

Interestingly, analogous conversions of 13 -hydroperoxides derived from LA and $\alpha$-LeA with BfEAS appeared to be more specific. Conversions of $13 \alpha$-HPOT yielded mainly the respective epoxy alcohol derivative (RT $4 \mathrm{~min}$ ) as product. Divinyl ether derivative could not be detected as side product. The only side product that was formed in those incubations was (9Z)-12-oxo-9-dodecenoic acid

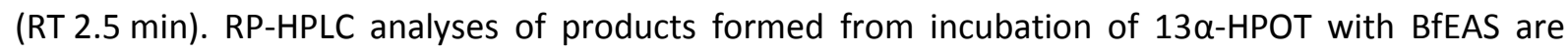
exemplarily shown in Figure 3.29.Product patterns of conversion of $\left[{ }^{14} \mathrm{C}\right]-13-\mathrm{HPOD}$ with BfEAS were similar to that of $\left[{ }^{14} \mathrm{C}\right]-13 \alpha$-HPOT (see appendix Figure $9.3 \mathrm{~B}$ ).

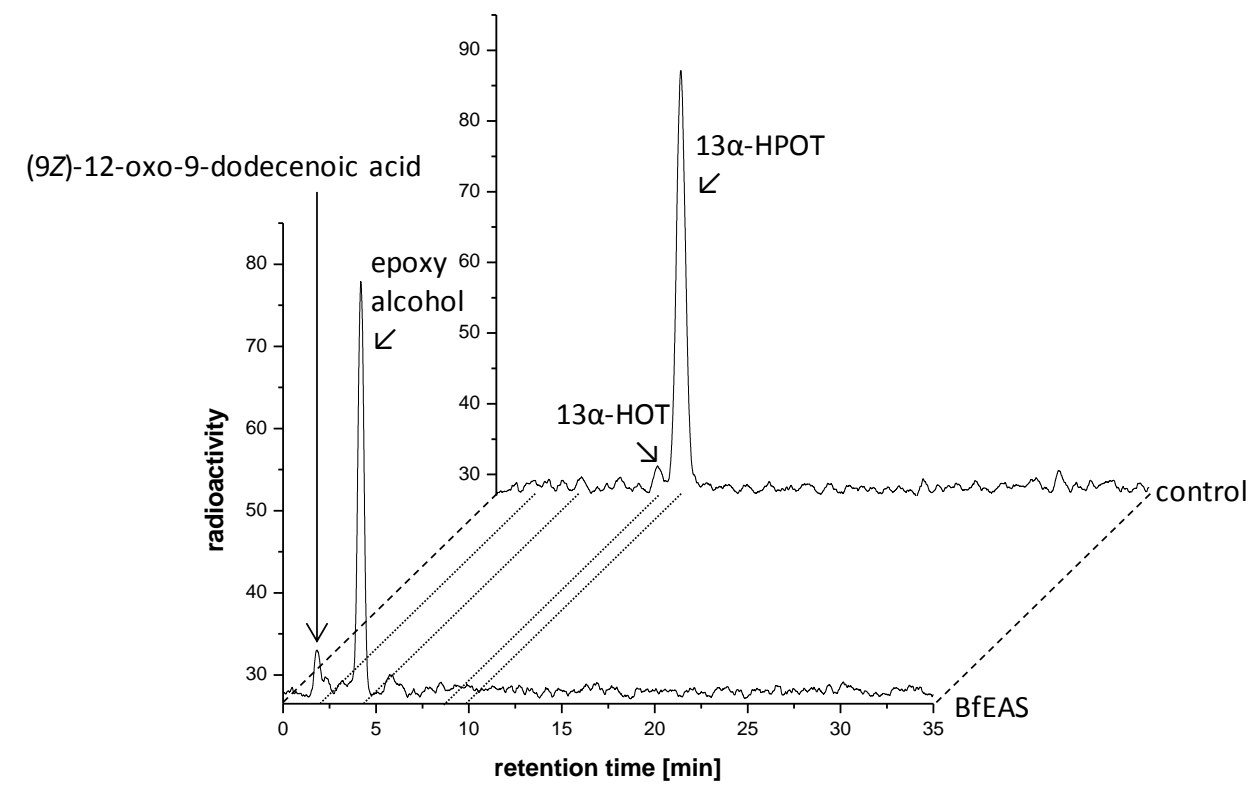

Figure 3.29: Product analysis from incubation of BfEAS with $\left[1-{ }^{14} \mathrm{C}\right]-13 \alpha-\mathrm{HPOT}$. The purified enzyme was incubated with the radio-labeled substrate for $30 \mathrm{~min}$ at RT. After extraction with diethyl ether the products were analyzed by RP-HPLC coupled to a radio-detector. The figure is representative for five experiments.

The occurrence of EAS-, HPL- and DES-specific products suggested close interconnection of the different enzymatic trajectories in CYP74-catalysis (Lee et al., 2008, Li et al., 2008, Brash, 2009,Cho et al., 2011).

Table 3.5 summarizes the products formed by incubations of BfEAS with the different radio-labeled 9-and 13-hydroperoxides derived from LA and $\alpha$-LeA. 
Table 3.5: Product specificities of BfEAS. Affinity purified enzymes were incubated with $\left[1-{ }^{14} \mathrm{C}\right]$-labeled hydroperoxy fatty acids for approx. $30 \mathrm{~min}$ at RT. After extraction with diethyl ether the isolated products were analyzed by RP-HPLC that was coupled to a radio-detector and quantified by integration of the respective peak area. The relative amounts of each product is indicated by the number of "+".

\begin{tabular}{lcccc}
\hline substrate & $\begin{array}{c}\text { w-oxo fatty acids } \\
\text { (= HPL activity) }\end{array}$ & $\begin{array}{c}\text { epoxy alcohols } \\
\text { (= EAS activity) }\end{array}$ & $\begin{array}{c}\text { divinyl ether } \\
\text { (= DES activity) }\end{array}$ & other products \\
\hline 9-HPOD & + & ++++ & + & n. d. \\
$9 \alpha-$ HPOT & ++ & +++ & ++ & + \\
$13-$ HPOD & + & +++++ & n. d. & n. d. \\
$13 \alpha-$ HPOT & + & +++++ & n. d. & - \\
\hline
\end{tabular}

\subsubsection{RP-HPLC/MS ${ }^{2}$}

In order to elucidate the exact structure of the different epoxy alcohol derivatives, the formed products were also analyzed by means of RP-HPLC/MS'. Therefore incubations of purified BfEAS with 9- and 13-hydroperoxides derived from LA and $\alpha$-LeA were performed as described in (2.3.13). The following figures exemplarily show extracted ion chromatograms as well as the respective tandem mass spectra with potential fragmentation patterns of the epoxy alcoholsderived from incubations of BfEAS with 9 $\alpha$-HPOT (Figure 3.30 and Figure 3.31) and 13 $\alpha$-HPOT (Figure 3.32).

In Figure 3.30 the extracted $m / z 309$ ion chromatogram (A) and tandem mass spectrum (B) for the conversion of BfEAS with $9 \alpha$-HPOT is shown. The fragment pattern is similar to that of known pattern reported before (Oliw et al., 2006). The $m / z 185$ and $m / z 201$ signals suggested that epoxide migration from $(9 S, 10 S, 12 Z, 15 Z)$-11-hydroxy-9,10-epoxy-12,15-octadecadienoic acid to (12Z,15Z)-9hydroxy-10,11-epoxy-12,15-octadecadienoic acid occurred as it has also been reported before by Oliw and coworkers (Oliw et al., 2006).

A

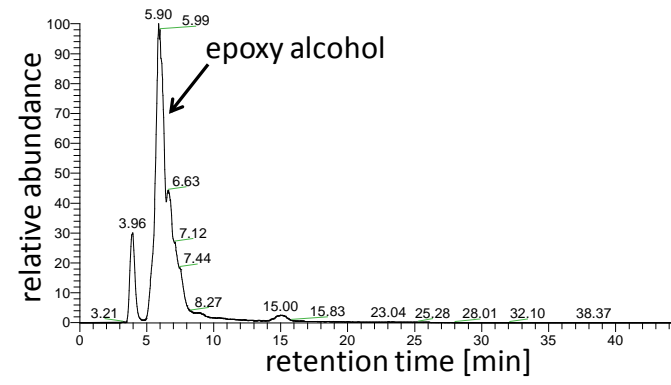

B

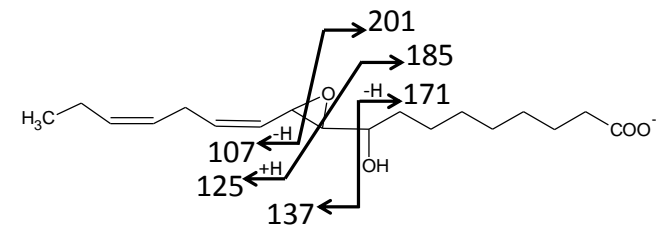

(12Z,15Z)-9-hydroxy-10,11-epoxy-12,15-octadecadienoic acid

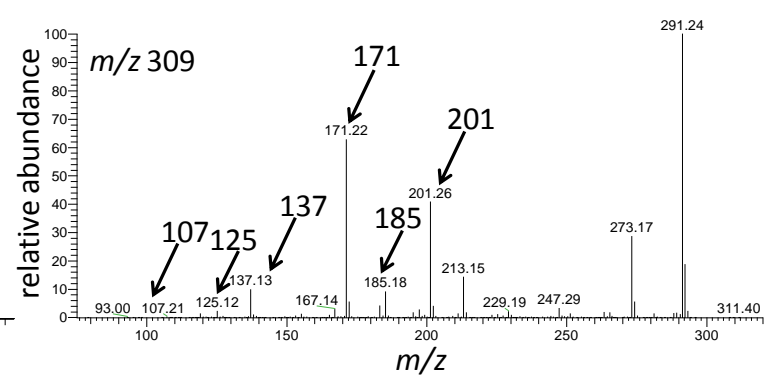

Figure 3.30: RP-HPLC/MS ${ }^{2}$-analysis of products formed by incubation of BfEAS with $9 \alpha-\mathrm{HPOT}$. Shown are the extracted ion chromatogram $(\mathrm{m} / \mathrm{z}$ 309) $(\mathrm{A})$ and the associated tandem mass spectrum of the epoxy alcohol(B). 
In order to confirm the formation of the divinyl ether derivative detected using radio-HPLC analysis and to analyze its chemical structure the respective product was pre-isolated via RP-HPLC/DAD and subsequently analyzed by RP-HPLC/MS². Theextracted $\mathrm{m} / \mathrm{z} 291$ ion chromatogram and the associated tandem mass spectrum and potential fragment pattern of the collected divinyl ether are shown in Figure 3.31. The collected peak was verified as colnelenic acid.

A

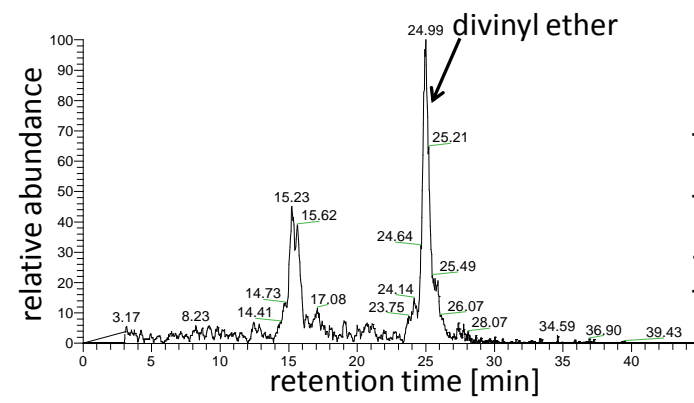

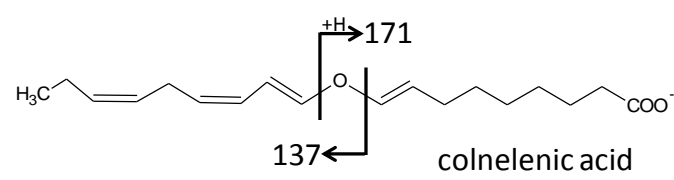

Figure 3.31: RP-HPLC/MS ${ }^{2}$-analysis of products formed by incubation of BfEAS with $9 \alpha-\mathrm{HPOT}$ (divinyl ether). Shown are the extracted $\mathrm{m} / \mathrm{z} 291$ ion chromatogram $(A)$ and the associated tandem mass spectrum (B) of the collected divinyl etherfraction of the RP-HPLC/DAD analysis.

The extracted $m / z 309$ ion chromatogram in Figure 3.32 shows the epoxy alcohol for the conversion of $13 \alpha$-HPOT. Its fragment pattern is in agreement with that of known pattern reported before (Cristea and Oliw, 2006).

A

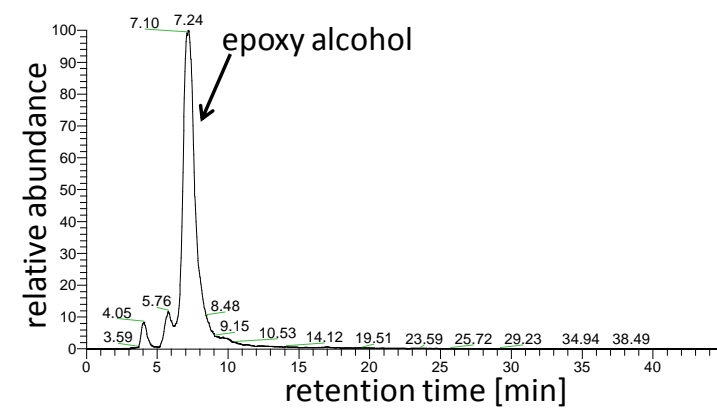

B

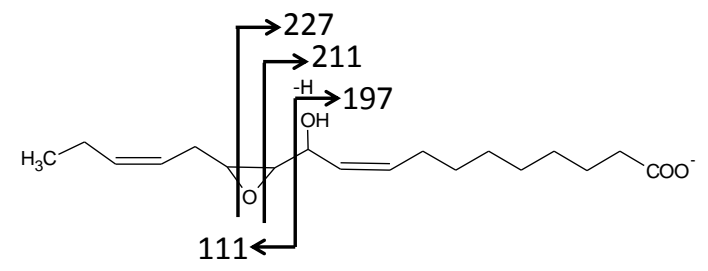

(9Z,15Z)-11-hydroxy-12,13-epoxy-9,15-octadecadienoic acid

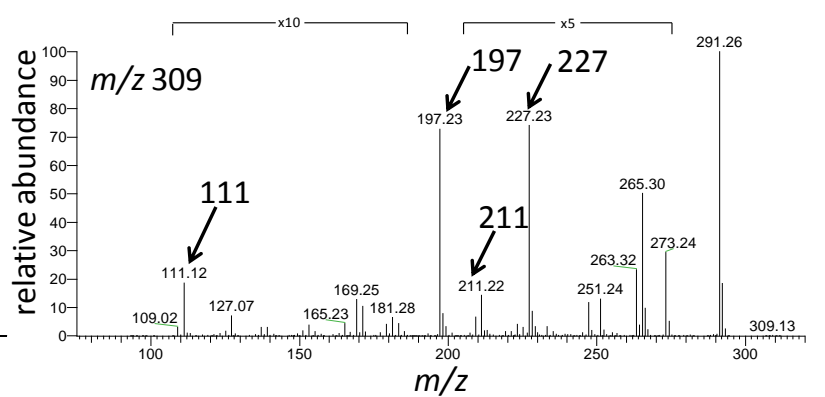

Figure 3.32: RP-HPLC/MS ${ }^{2}$-analysis of products formed by incubation of BfEAS with $13 \alpha-\mathrm{HPOT}$. Shown are the extracted ion chromatogram ( $\mathrm{m} / \mathrm{z}$ 309) (A)and the associated tandem mass spectrum of the epoxy alcohol (B).

Various fatty acid hydroperoxide isomers derived from $\mathrm{C}_{22}$ fatty acid DHA may be generated by the conversion with different LOXs-isoforms (Figure 3.27).In this study LOXs from soybean (GmLOX1), 
moss (PpLOX2), Arabidopsis (AtLOX1) and potato (StLOX), were used in order to produce different hydroperoxy isomers as potential substrates for BfEAS. Incubations were performed as described in 2.3.13 and the products were analyzed by RP-HPLC/MS ${ }^{2}$ (2.3.13.3).

In Figure 3.33 extracted ion chromatogram and associated tandem mass spectrum and potential fragment pattern of reaction of GmLOX1 with DHA are exemplarily shown.

Conversion of DHA by GmLOX1 led to the formation of 17-HDHA (Figure $3.33 \mathrm{~A}$ and $\mathrm{B}$ ) and the respective hydroperoxide 17-HPDHA.

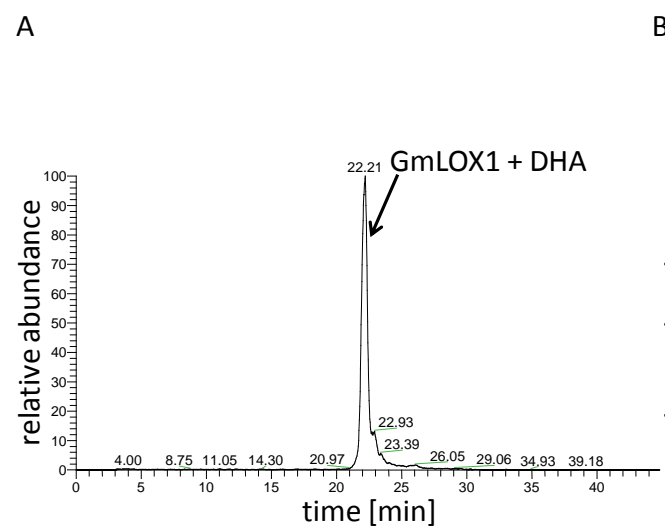

B
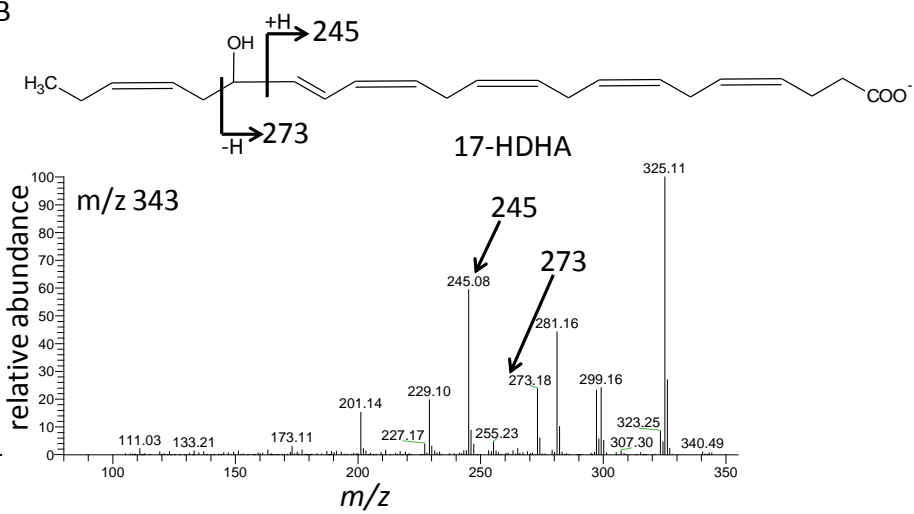

Figure 3.33: RP-HPLC/MS ${ }^{2}$-analysis of products formed by incubation of GmLOX1 with DHA. Shown are the extracted ion chromatogram $\mathrm{m} / \mathrm{z} 343$ and the associated tandem mass spectrum of 17-HDHA.Abbreviations: 17-HPDHA: 17-hydroperoxy docosahexaenoic acid.

In Table 3.6 products formed from reactions of DHA with different LOXs are summarized.Conversions of AtLOX1 and StLOX with DHA yielded 10-H(P)DHA, whereas PpLOX led to the formation of 14H(P)DHA.

Table 3.6: Product formation of conversions by various LOXs with DHA.

\begin{tabular}{cc}
\hline enzyme & hydro(pero)xides derived from DHA \\
\hline AtLOX1 & $10-\mathrm{H}(\mathrm{P}) \mathrm{DHA}$ \\
GmLOX1 & $17-\mathrm{H}(\mathrm{P}) \mathrm{DHA}$ \\
PpLOX & $14-\mathrm{H}(\mathrm{P}) \mathrm{DHA}$ \\
StLOX & $10-\mathrm{H}(\mathrm{P}) \mathrm{DHA}$ \\
\hline
\end{tabular}

The extracted ion chromatograms and associated tandem mass spectra and potential fragment patterns of 10-, 14- and 17-HDHA were consistent to known patterns (Hong et al., 2007).

After identification of possible isomers of conversions of DHA by various LOXs which can be used as substrates, analysis of product formations of incubations of the different LOXs with DHA and BfEAS were also analyzed. InFigure 3.34exemplarily the analysis of the reaction products from coincubation of DHA with GmLOX1 and BfEAS is shown. Extracted ion chromatogram and associated tandem mass spectrum and potential fragment pattern indicated formation of epoxy alcohol that 
was tentatively assigned as $(4 Z, 7 Z, 10 Z, 13 Z, 19 Z)-17$-hydroxy-15,16-epoxy-4,7,10,13,19-

docosapentaenoic acid as product.

A
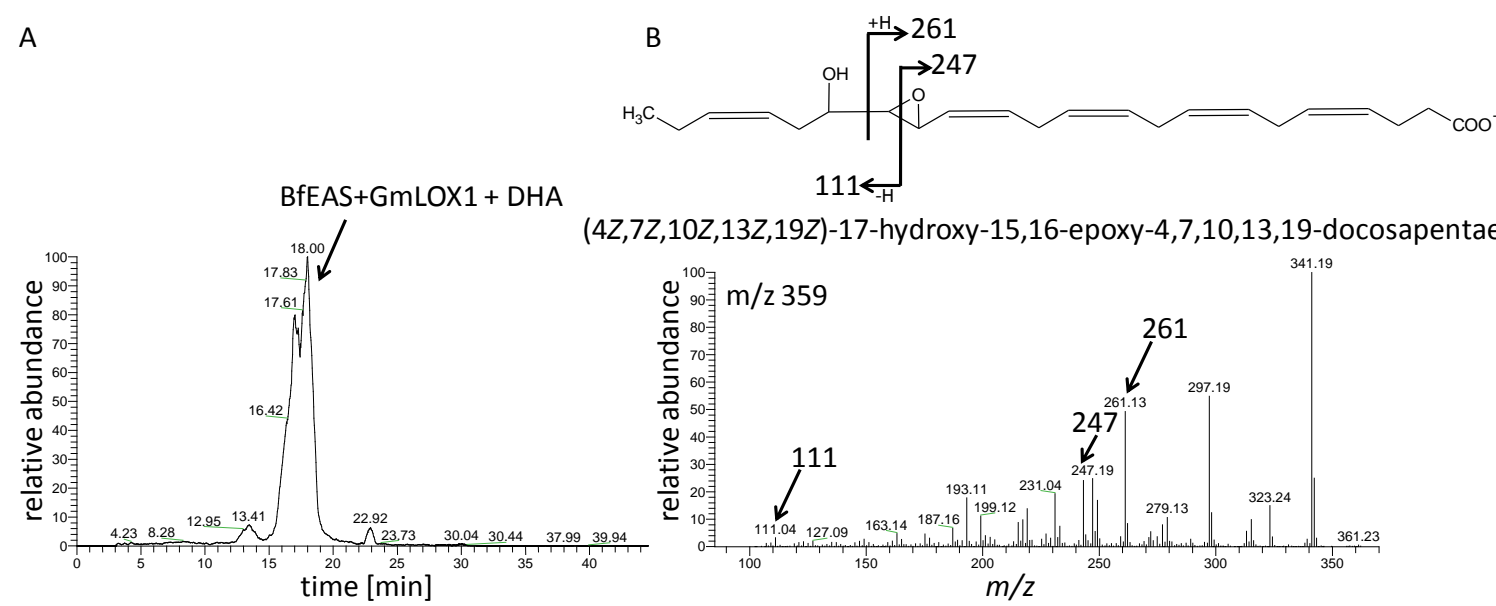

(4Z,7Z,10Z,13Z,19Z)-17-hydroxy-15,16-epoxy-4,7,10,13,19-docosapentaenoic acid

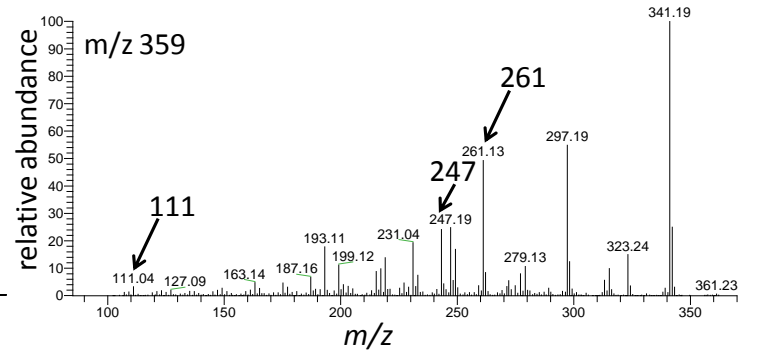

Figure 3.34: RP-HPLC/MS ${ }^{2}$-analysis of products formed by incubation of GmLOX1 with DHA and BfEAS. Shown are the extracted $m / z 359$ ion chromatogram (A) and the associated tandem mass spectrum (B).

Conversions of DHA with AtLOX1, StLOX and PpLOX, respectively, and BfEAS led as well with GmLOX to the formation of epoxy alcohols as products.

Co-incubations of DHA with AtLOX1 and BfEASlead to formation of an epoxy alcohol that was tentatively assigned as $(4 Z, 7 Z, 13 Z, 16 Z, 19 Z)$-10-hydroxy-11,12-epoxy-4,7,13,16,19-docosapentaenoic acid. The extracted ion chromatogram and associated tandem mass spectrum and potential fragment patterns are shown in Figure 3.35.

A
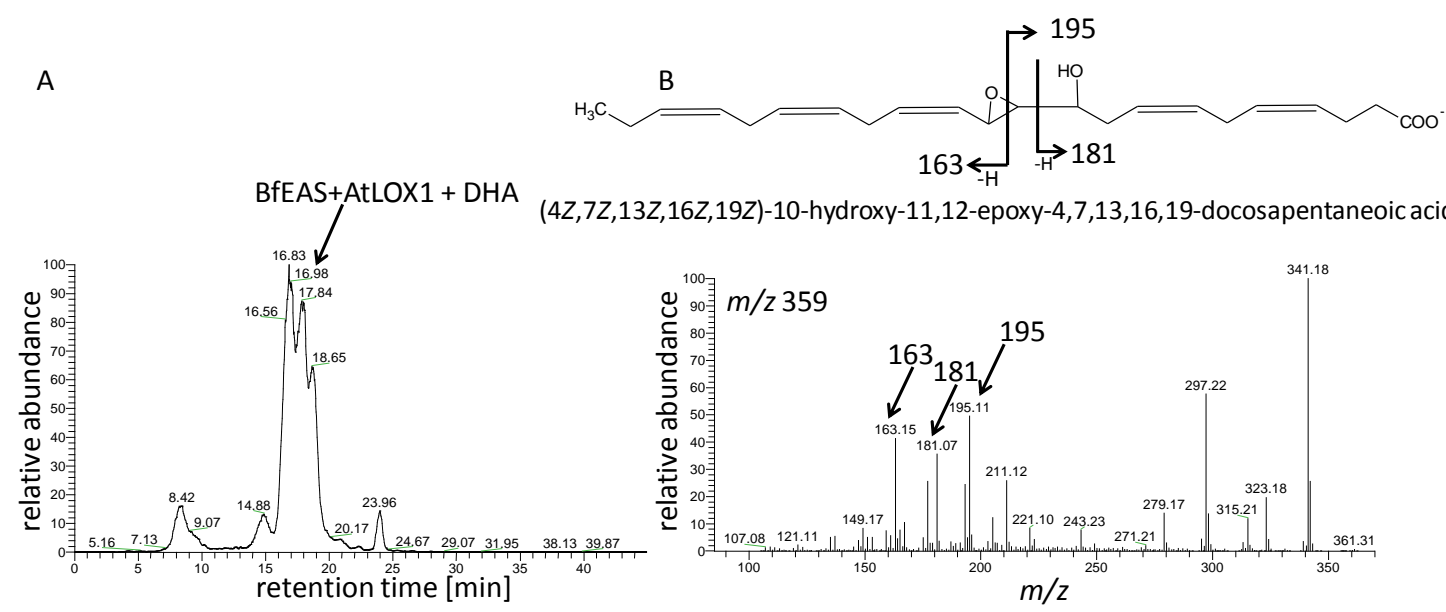

Figure 3.35: RP-HPLC/MS ${ }^{2}$-analysis of products formed by incubation of AtLOX1 with DHA and BfEAS. Shown are the extracted $m / z 359$ ion chromatogram (A) and the associated tandem mass spectrum (B).

Like AtLOX1 the StLOX led also to the formation of $(4 Z, 7 Z, 13 Z, 16 Z, 19 Z)$-10-hydroxy-11,12-epoxy$4,7,13,16,19-$ docosapentaenoic acid by co-incubation with BfEAS and DHA.The extracted ion chromatogram and associated tandem mass spectrum and potential fragment pattern are shown inFigure 3.36 . 
A

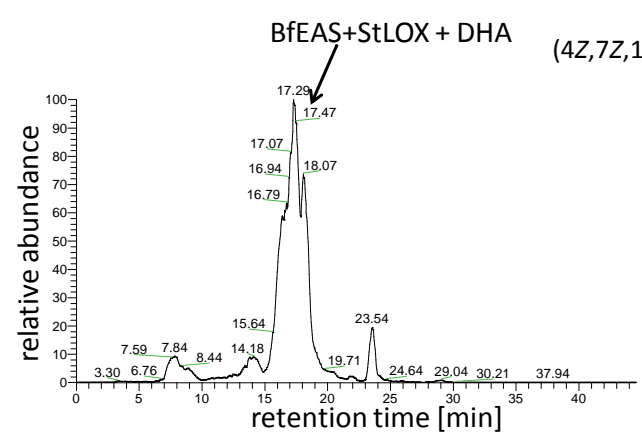

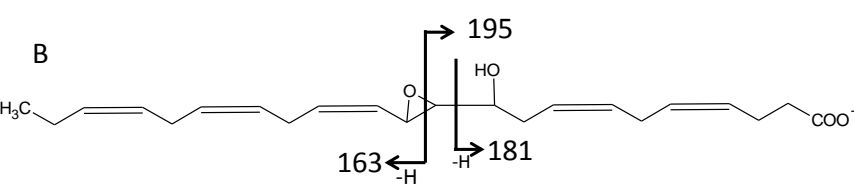

,7Z,13Z,16Z,19Z)-10-hydroxy-11,12-epoxy-4,7,13,16,19-docosapentaneoic acid

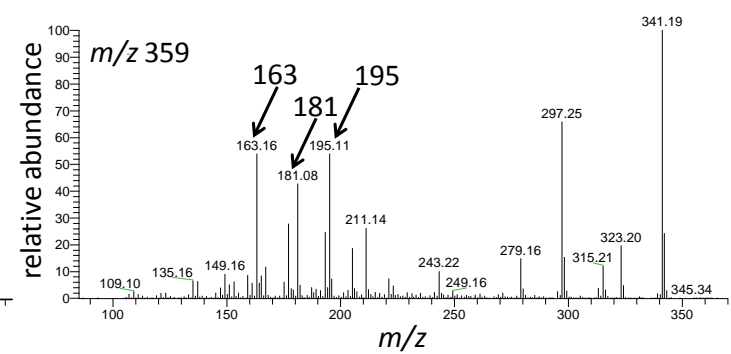

Figure 3.36: RP-HPLC/MS ${ }^{2}$-analysis of products formed by incubation of StLOX with DHA and BfEAS. Shown are the extracted $m / z 359$ ion chromatogram (A) and the associated tandem mass spectrum (B).

Figure 3.37 shows the extracted ion chromatogram and associated tandem mass spectrum and potential fragment pattern of co-incubation of BfEAS with PpLOX and DHA. The formed epoxy alcohol was tentatively assigned as $(4 Z, 7 Z, 10 Z, 16 Z, 19 Z)-14$-hydroxy-12,13-epoxy-4,7,10,16,19docosapentaenoic acid as product.

A

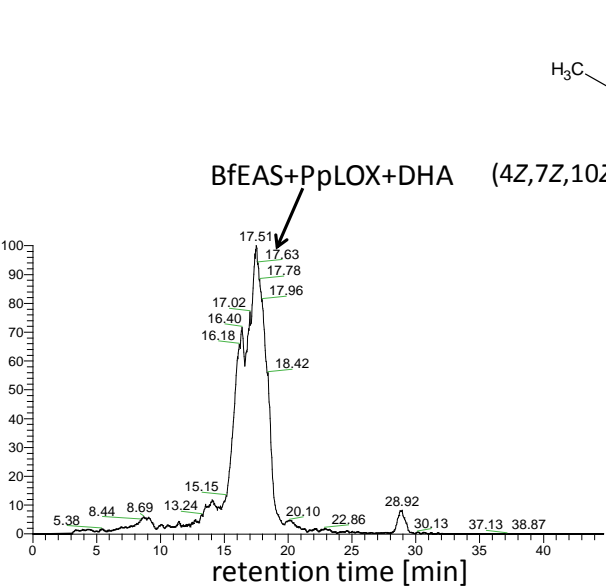

B

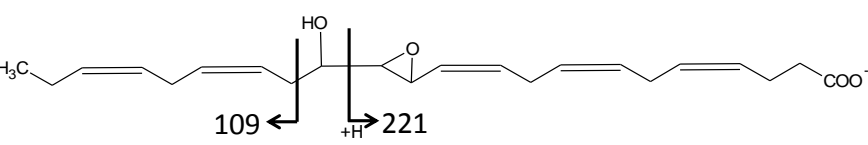

Figure 3.37: RP-HPLC/MS ${ }^{2}$-analysis of products formed by incubation of PpLOX with DHA and BfEAS. Shown are the extracted $m / z 359$ ion chromatogram (A) and the associated tandem mass spectrum (B).

\subsubsection{Kinetic analysis}

DHA leads to the formation of ten positional isomers which may be used as substrates (Figure 3.27). Therefore it is of interest to determine substrate preference of conversions of BfEAS with hydroperoxides derived from $\mathrm{C}_{18}$ - and $\mathrm{C}_{22}$ fatty acids by spectrometrically approaches (2.3.11.1). 


\subsubsection{1 pH optimum}

To determine the optimal buffer conditions for measurements of the kinetic parameters first the pH optimum of EAS was measured as described in 2.3.14.1. Therefore the initial velocity for the reaction of $1 \mathrm{nM}$ BfEAS with $30 \mu \mathrm{M} 13-\mathrm{HPOD}$ at $234 \mathrm{~nm}$ was measured in different buffers. A citratephosphate buffer was used for the $\mathrm{pH}$ range 2.82 to 7.25 , phosphate buffer for the $\mathrm{pH}$ range 5.5 to 8.0, Tris buffer for the range 7.31 to 9.22 and a glycine- $\mathrm{NaOH}$ buffer for the range from 8.71 to 9.9 . The optimal $\mathrm{pH}$ for the BfEAS was determined to be around $\mathrm{pH} 6.75$, which can be seen in Figure 3.38 .

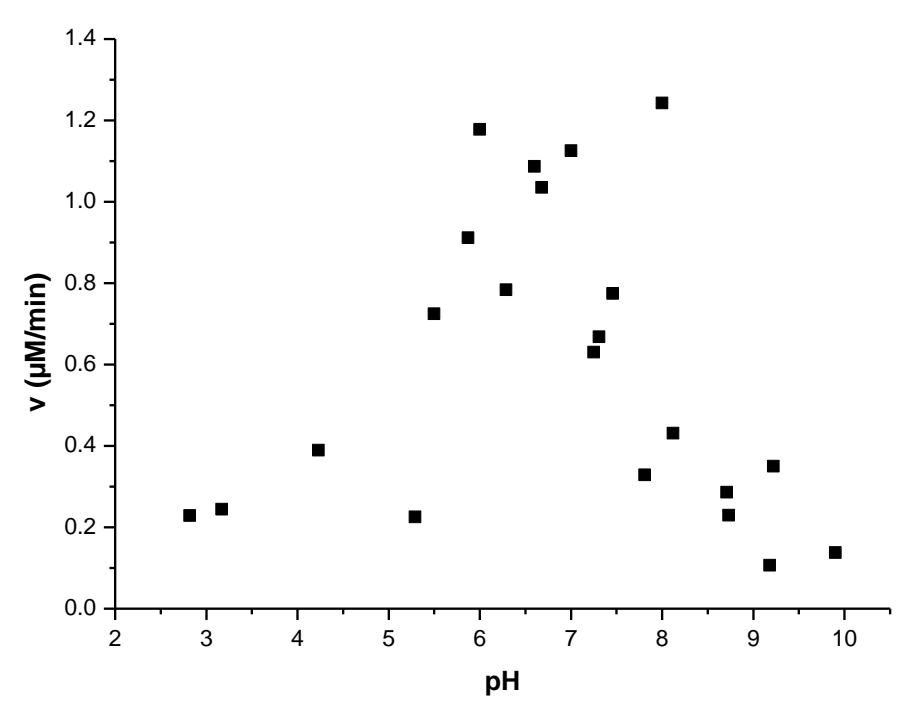

Figure 3.38: The pH optimum of BfEAS with 13-HPOD. The initial velocity for the conversion of $1 \mathrm{nM}$ BfEAS with $30 \mu \mathrm{M} 13-\mathrm{HPOD}$ at $234 \mathrm{~nm}$ in different buffers at RT was measured. Each data point represents the mean value of three measurements.

\subsubsection{Kinetic parameters}

To determine the substrate preference of BfEAS kinetic parameters for reactions of BfEAS with 13hydroperoxides derived from $C_{18}$ fatty acids $L A$ and $\alpha$-LeA, and for reaction of BfEAS with 17hydroperoxide derived from $C_{22}$ fatty acid DHA were measured as described (2.3.11.1). The reaction was started by addition of $1 \mathrm{nM}$ BfEAS.

The kinetic parameters were determined by plotting the derived data points to the MichaelisMenten equation.

The Michaelis-Menten diagrams for the reactionsof BfEAS with 13-HPOD and 13 $\alpha$-HPOT, respectively, where the initial time-dependent changes at $234 \mathrm{~nm}$ were measured, are shown in Figure 3.39. 
A

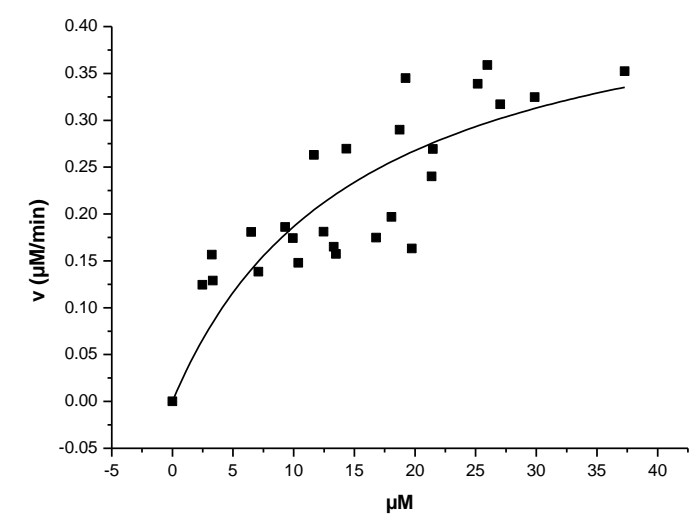

B

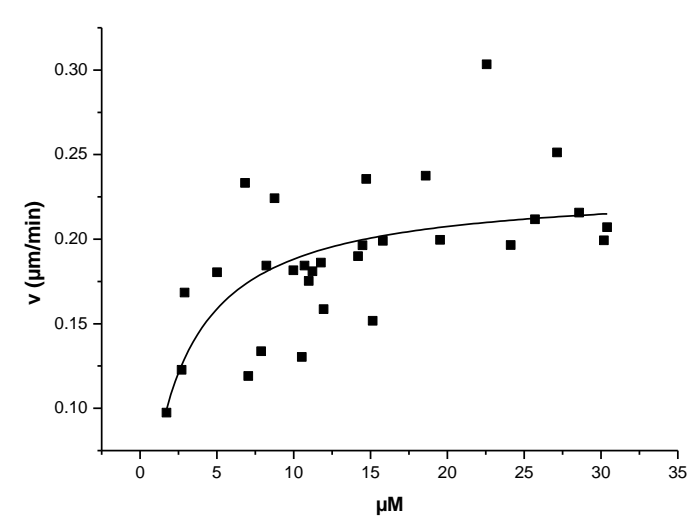

Figure 3.39: Kinetic analyses of BfEAS with 13-HPOD (A) and $13 \alpha-$ HPOT (B). The reactions were performed in $50 \mathrm{mM}$ sodium phosphate buffer, $\mathrm{pH} 6.5$ at RT and were started by addition of $1 \mathrm{nM}$ BfEAS. The initial timedependent changes at $234 \mathrm{~nm}$ were measured.For each data point the substrate concentration was measured again.

InFigure 3.40 the Michaelis-Menten diagram derived from measuring the initial time-dependent substrate consumption at $234 \mathrm{~nm}$ for the reaction of BfEAS with $\mathrm{C}_{22}$ fatty acid derived hydroperoxide 17-HPDHA is shown.

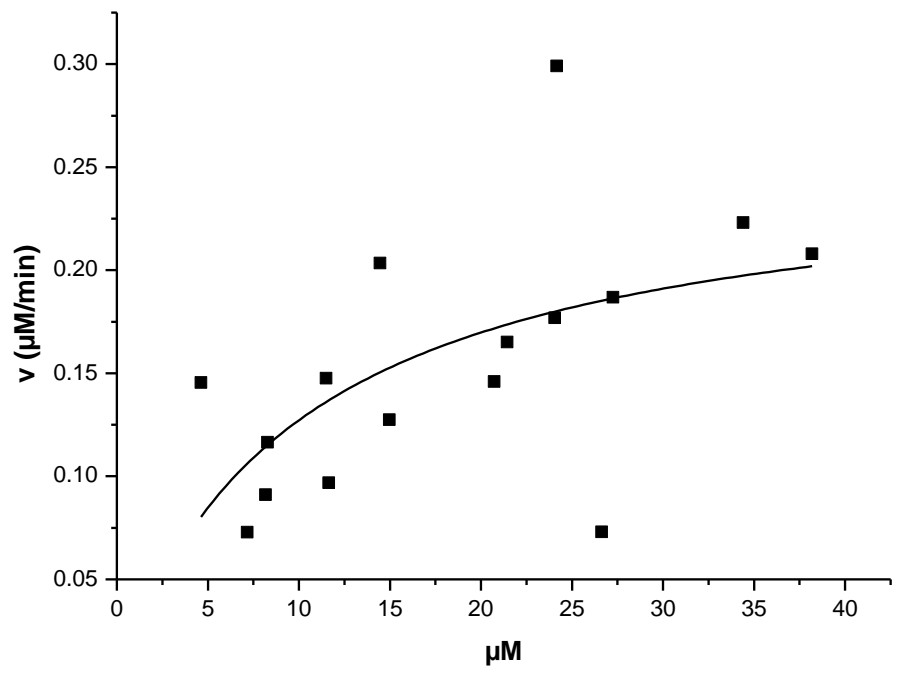

Figure 3.40: Kinetic analysis of BfEAS with 17-HPDHA.The reaction was performed in $50 \mathrm{mM}$ sodium phosphate buffer, pH 6.5 at RT and was started by addition of $1 \mathrm{nM}$ BfEAS. The initial time-dependent changes at $234 \mathrm{~nm}$ were measured.For each data point the substrate concentration was measured again.

Table 3.7 summarizes the derived kinetic parameters. The $\mathrm{k}_{\mathrm{cat}}$-values were calculated by considering heme occupancy of approx. $37 \%$. 
Table 3.7: Kinetic parameters for the reaction of BfEAS with different hydroperoxides from $\mathrm{C}_{18^{-}}$and $\mathrm{C}_{22}$ fatty acids. The kinetic parameters were determinedby measuring the initial time-dependent substrate consumption at $234 \mathrm{~nm}$ at different substrate concentrations. For analysis between 15 and 25 data points were fitted to the Michaelis-Menten equation. The concentration of BfEAS was $1 \mathrm{nM}$. The values for $\mathrm{k}_{\mathrm{cat}}$ were calculated by considering heme occupancy of approx. $37 \%$.

\begin{tabular}{|c|c|c|c|c|}
\hline substrate & $\begin{array}{c}\mathrm{K}_{\mathrm{M}} \\
{[\mu \mathrm{M}]}\end{array}$ & $\begin{array}{c}V_{\max } \\
{[\mu \mathrm{M} / \min ]}\end{array}$ & $\begin{array}{c}\mathbf{k}_{\text {cat }} \\
{[1 / \mathrm{min}]}\end{array}$ & $\begin{array}{c}\mathbf{k}_{\text {cat }} / K_{M} \\
{\left[\min ^{-1} \mathrm{M}^{-1} \times 10^{6}\right]}\end{array}$ \\
\hline 13-HPOD & $\begin{array}{c}15 \\
+/-6\end{array}$ & $\begin{array}{c}0.5 \\
+/-0.09\end{array}$ & 1351 & 90 \\
\hline $13 \alpha$-НРОТ & $\begin{array}{c}2 \\
+/-0.9\end{array}$ & $\begin{array}{c}0.23 \\
+/-0.02\end{array}$ & 622 & 311 \\
\hline 17-HPDHA & $\begin{array}{c}10 \\
+/-7\end{array}$ & $\begin{array}{c}0.25 \\
+/-0.0\end{array}$ & 676 & 68 \\
\hline
\end{tabular}

The values for $\mathrm{k}_{\mathrm{cat}} / \mathrm{K}_{\mathrm{M}}$ showed that BfEAS isomerized both 13-hydroperoxides derived from LA and $\alpha$ LeA and 17-hydroperoxide derived from DHA with specificity for $13 \alpha-$ HPOT.

Kinetic measurements of BfEAS with 9-hydroperoxides derived from LA and $\alpha$-LeA turned out to be very difficult since divinyl ethers were also formed in significant amount besides the epoxy alcohol derivative (Figure 3.28). As these compounds have relatively broad absorption maxima at $252 \mathrm{~nm}$ (CA, EA and EnA) to $268 \mathrm{~nm}(\mathrm{EnA})$ that overlap with that of the hydroperoxide substrate at $234 \mathrm{~nm}$ no reasonable data were obtained in this analysis. Measurements of the kinetic parameters with other hydroperoxides derived from DHA (10- and 14-HPDHA) were not performed, sinceit was not possible to produce enough of these substrates.

\subsubsection{Site-directed mutagenesis}

Sequence alignments (Figure 3.41) showedthat in BfEAS particular amino acid-residues which are important for product specificity in AtAOS are also conserved in BfEAS as it has also been reported before (Lee et al., 2008). At position 58 BfEAS contains a strictly conserved (F), which is AOS-specific. Analysis of products of an analog PpAOS1 variant (PpAOS1_F93L) where this conserved $F$ was substituted by $L$ showed that the AOS altered its product activity to HPL activity (see 3.1.6.2.1). To analyze product activity and importance of this conservedF in BfEAS it was substituted by the essential residue for HPL activity L (BfEAS_F58L). Also, at position 75 BfEAS contains a strictly conserved proline (P). This residue was substituted by a HPL-specific A (BfEAS_P75A) and also by an AOS-specific S (BfEAS_P75S) (Figure 3.41 A). 


\section{A}

\begin{tabular}{l} 
AtAOS \\
\hline PaAOS \\
LeAOS3 \\
PpAOS1 \\
PpAOS2 \\
AtHPL \\
LeHPL \\
PpHPL \\
StDES \\
AsDES \\
BfEAS \\
BfEAS_1 \\
BfEAS_2
\end{tabular}

DKVEKKDIETGTYMPSTELTGGYRIISYLDPSEPK 163 SKVEKKDIFTGTYMPSTKLTGAYRVI SYLDPSEPR 118 SQVDKENYFEGTFMSSPSFNGGYKVCGFLGTSDPK 134 SKVEKKDVFTGTYMPSVSFTSGYRVCSYLDPSEER 119 NKCEKRDI FLGTYMPDLSYTSGHRVI SYLDPSEVR 119 DLVDKRDVII IGDFRPSLGFYGGVRVGVYLDTTEPK 36 EIVEKANVIVGDFMPSVVYTGDMRVCAYLDTSEPK 127 DKVDKYKAFAGTIMPSTEYNGGYEVCAYLDASDKK 177 SLIDKTDTLGGTFKPGKEYYSGYRPVAFIDTKDPN 122 SKVSKVNSITGNYMPALSFTGGYRVCAYLDPSEPT 123 IELFKKEEF CFGVA-EVRRDFTEGICPSVSSNGKI 82 IDLFKKEEFCFGVVGEVRKDFTEGVCPCILSNGKI 84

VDLFRKEEFYFGPI-EVRKDFTEGVCPSITSNGKI 206
B

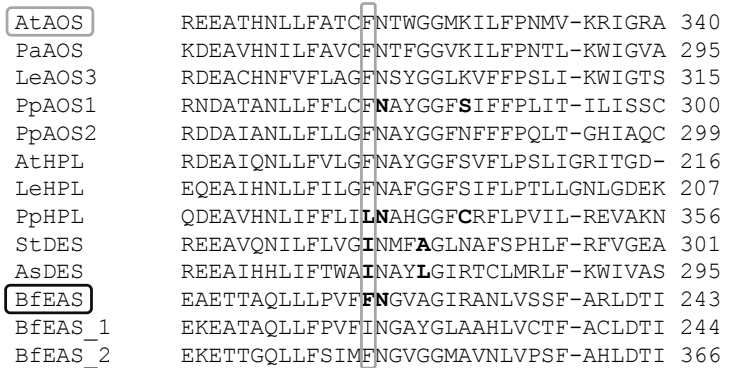

Figure 3.41:Partial multiple sequence alignment of different CYP74 enzymes from different plant species and marine invertebrates. Allium sativum (As); Arabidopsis thaliana (At); Branchiostoma floridae (Bf); Lycopersicum esculentum (Le); Parthenium argentatum (Pa); Physcomitrella patens (Pp); Solanum tuberosum (St); AtAOS, CAA63266; AsDES, AJ867809; AtHPL, AAC69871; BfEAS, EU555186; BfEAS_1, XP2601390; BfEAS_2, XP2602690; LeAOS3, AAN76867; LeHPL, AAF67142; PaAOS, CAA55025; PpAOS1,CAC86919; PpAOS2, XP_001759629; PpHPL, CAC86920; StDES, CAC28152. The highly conserved residues are in grey brackets. Amino acid positions used for site-directed mutagenesis in this study are marked in bold letters.

Additionally to the BfEAS_F58L variant a BfEAS_F60L variant was generated. Lee and coworkers (Lee et al., 2008) showed in alignments and structures that the particular F which is highly conserved and determining enzymeactivity was located at position 60. Sequence alignments in this study showed that this special $F$ might be the $F$ located at position 58 (Figure $3.41 \mathrm{~A}$ ). To determine which $F$ is the specific residue variants for both substitutions (F58L and F60L) were generated.

As reported before for tomato AOS (LeAOS3_F295) (Toporkova et al., 2008) a further F is strictly conserved in AOS and HPLS. In DES an isoleucin (I) is located at this position. Substitutions of this conserved $\mathrm{F}$ by I (BfEAS_F223I) and by PpHPL-specific L (BfEAS_F223L) (Figure 3.41 B) were performed (2.3.1.1).

According to site-directed mutagenesis in AtAOS (AtAOS_N321Q) BfEAS_N224Q variant was generated. This substitution should abolish enzyme activity (Lee et al., 2008).

A very special feature of the CYP74-enzymes is that they contain in contrast to typical P450-enzymes an insertion of 9 amino acids in the heme-bindingloop (Lee et al., 2008). In order to analyze the molecular function of this unique feature and its impact on catalysis, a truncation variant of BfEAS was generated lacking these nine amino acids (Figure 3.42). 


\begin{tabular}{|c|c|c|}
\hline & 9AS-insert & \\
\hline & $\mathrm{x} \times \mathrm{x} \mathrm{CxG}$ & \\
\hline AtAOS & RHVLWSNG $\overline{\text { PETETPTVGNKQCAGK--D }}$ & 475 \\
\hline PaAOS & KYVWWSNGPETESPTVENKQCAGK--D & 430 \\
\hline LeAOS3 & KYVYWSNGKEIDNPSVNDKQCPGK--D & 448 \\
\hline PpAOS1 & KYIFWSNCYETDEPTTANKQCAGK--D & 435 \\
\hline PpAOS2 & GNVFWSNGRETDDPTVHDKQCAGK--D & 434 \\
\hline AtHPL & NYLYWSNGPQTGTPSASNKQCAAK--D & 350 \\
\hline LeHPL & NYLFWSNGPQTGRPTESNKQCAAK--D & 442 \\
\hline PpHPL & AHLVWSNGRQTDETTVYIKQCAGK--E & 493 \\
\hline StDES & KHVLWSNGRETENPAPDNKQCPGK--D & 435 \\
\hline AsDES & KHVLWANCYGTDAPKADDKICAGK--D & 429 \\
\hline BfEAS & KHHVIFHCRLTDTPAVNNHMCPGRDVS & 383 \\
\hline BfEAS_1 & KHHVTFHCRLTDTPAVNNHMCPGKDVS & 384 \\
\hline $\mathrm{BfEAS}^{-} 2$ & EHHVTFHCRLIDTPATNNHMCPGKDVG & 506 \\
\hline
\end{tabular}

Figure 3.42:Partial multiple sequence alignment of different CYP74 enzymes from different plant species and marine invertebrates. Allium sativum (As); Arabidopsis thaliana (At); Branchiostoma floridae (Bf); Lycopersicum esculentum (Le); Parthenium argentatum (Pa); Physcomitrella patens (Pp); Solanum tuberosum (St); AtAOS, CAA63266; AsDES, AJ867809; AtHPL, AAC69871; BfEAS, EU555186; BfEAS_1, XP2601390; BfEAS_2, XP2602690; LeAOS3, AAN76867; LeHPL, AAF67142; PaAOS, CAA55025; PpAOS1,CAC86919; PpAOS2, XP_001759629; PpHPL, CAC86920; StDES, CAC28152. The additional 9 amino acid insertion is in grey brackets. Amino acids removed by mutagenesis in this study are marked in bold letters.

In analogy to the site directed mutagenesis of PpAOS1 and PpHPL described before (3.1.6) BfEAS was mutated. Therefore the activity determining amino acid residues in BfEAS were changed or removed (Figure 3.41 and Figure 3.42).

\subsubsection{Purification}

BfEAS variants were purified as described in 2.3.8.1. The SDS-PAGEanalyses of the different variants (data not shown) were similar to that of wild type (Figure 3.24). However, SDS-PAGE analysis of the BfEAS_9AS-deletion variant showed multiple smaller bands (Figure 3.43). At $66 \mathrm{kDa}$ the protein band of BfEAS is shown. Beside this band there were also strong protein bands at $55 \mathrm{kDa}, 57 \mathrm{kDa}$, and $60 \mathrm{kDa}$. It seemed that these bands belong to the eluted BfEAS and weren't unspecifically bound proteins which were eluted with the bound BfEAS. 


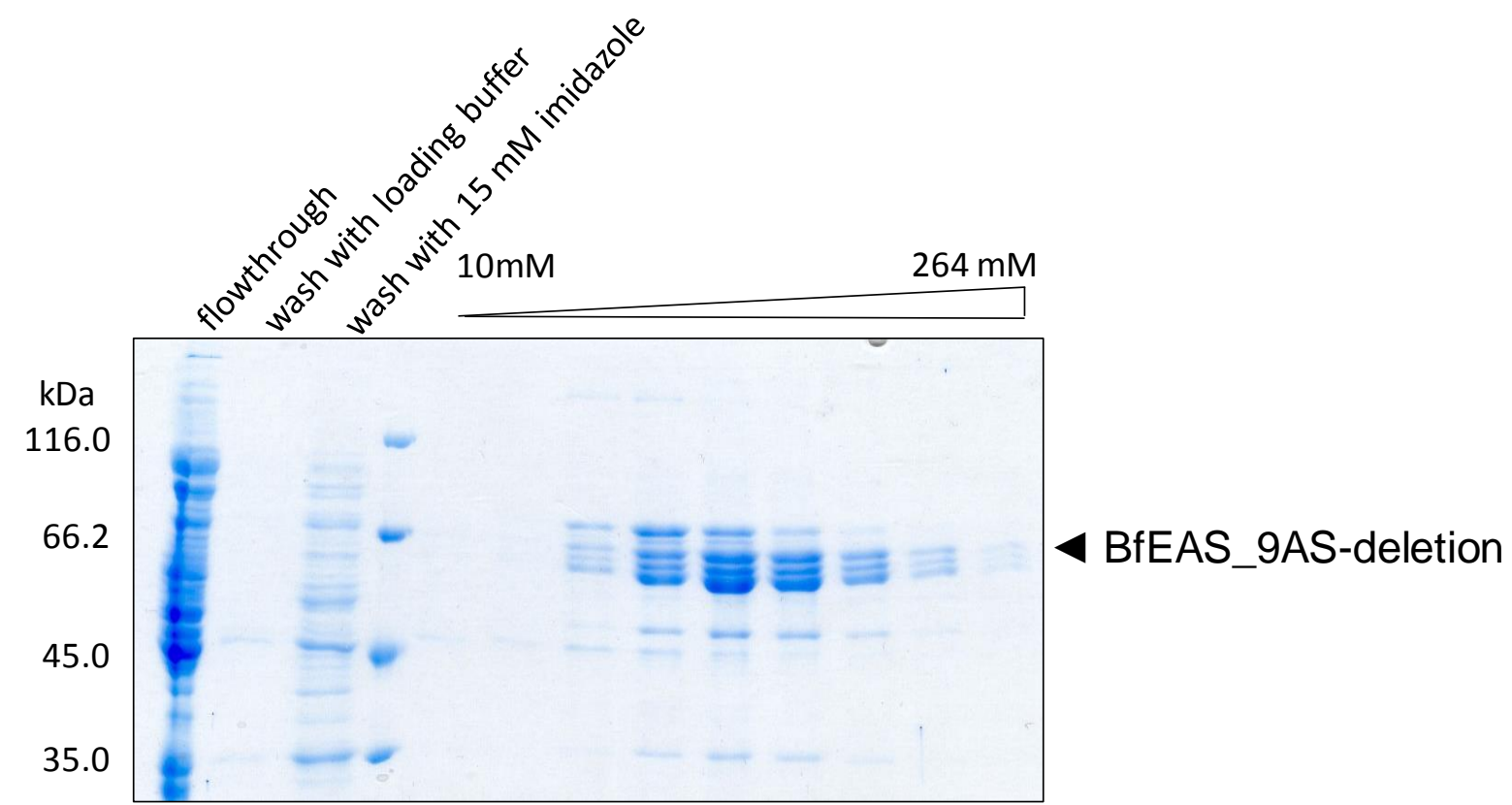

Figure 3.43: SDS-PAGE analysis of purified BfEAS_9AS-deletion. The enzyme was heterologously expressed in BL21 Star. The cells were harvested and disrupted by sonification. The cell debris were removed by centrifugation. The purification was done by $\mathrm{Ni}^{2+}$-affinity chromatography. The column was first washed with loading buffer ( $50 \mathrm{mM}$ sodium phosphate, $\mathrm{pH} 8.0,1 \mathrm{M} \mathrm{NaCl}, 500 \mathrm{mM}$ urea) and then with buffer which contains additionally $15 \mathrm{mM}$ imidazole. The protein was eluted from the column by a linear gradient with an increasing imidazole concentration (15 mM to $300 \mathrm{mM}$ ).Aliquots of collected fractions of the purification were loaded on a $10 \%$ SDS-PAGE gel.The figure is representative for two purifications.

Similar to the purification of wild type enzyme by affinity chromatography, very high amounts of homogenous protein were isolated from E. coli expressing the different variant enzymes (about to $30 \mathrm{mg}$ protein/ml expression culture). The purified proteins were used for further analysis.

\subsubsection{Spectroscopic analysis}

To investigate if the above mentioned substitutions of special amino acid residues have an effect on the protein folding and cofactor incubation, UV/vis and CD spectroscopy analysis were performed as described (2.3.15).

\subsection{UV/vis analysis}

UV/vis spectra ofBfEAS variants which werecompared to that of the wild type enzyme are shown in Figure 3.44. BfEAS_F58L (A) had absorption maxima at $420 \mathrm{~nm}$ (Soret), $281 \mathrm{~nm}(\gamma)$ and a very small at $348 \mathrm{~nm}(\delta)$. Absorption maxima for the $\alpha$ - and $\beta$-bands were not measured.The absorption maxima of BfEAS_9AS-deletion were at $423 \mathrm{~nm}$ (Soret), $280 \mathrm{~nm}(\gamma)$ and at $356 \mathrm{~nm}(\delta)$ (B). There was a small maximum at $546 \mathrm{~nm}(\beta)$ and no absorption maximum for $\alpha$-band. 
A

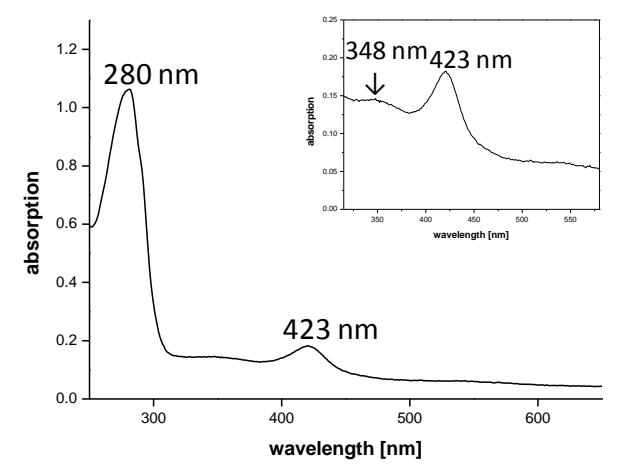

C

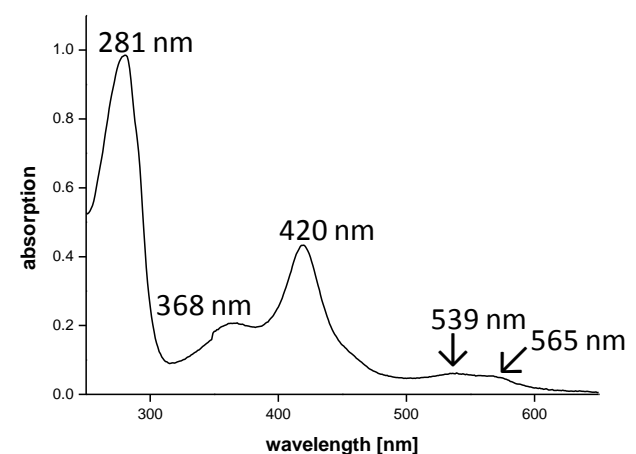

B

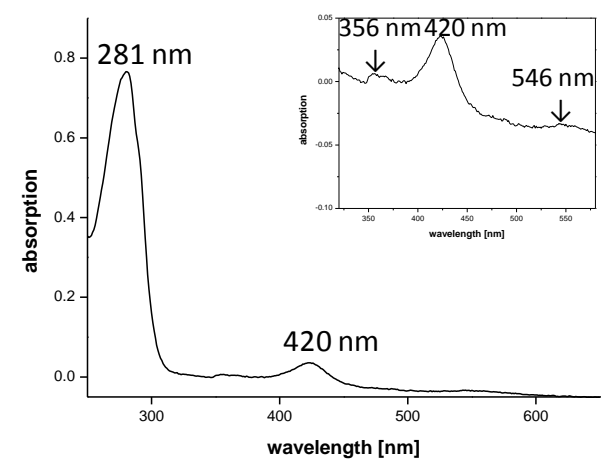

Figure 3.44: UV/vis spectra of purified BfEAS_F58L (A) and BfEAS_9AS-deletion (B) in comparison to the BfEAS wild type (C). The spectra were measured in $50 \mathrm{mM}$ sodium phosphate buffer, $\mathrm{pH}$ 6.0.The spectraare representative for one to three experiments.

The difference between mutants and wild type is that the mutants did not show absorption maxima for $\alpha$ - and $\beta$-bands (BfEAS_F58L) or even very small absorption maximum only for $\beta$-band (BfEAS_9AS-deletion). Maxima for $\delta$-band were also very small in the variants. Another difference is in the heme content. The heme content of BfEAS_F58L is of $16 \%$ and of BfEAS_9AS-deletion of $2 \%$, whereas the content of wild type of $37 \%$ was. Therefore the variants had lower heme contents than the wild type especially the 9AS-deletion variant, where the amino acid insertion near the hemebinding loop was deleted. 


\subsection{CD spectroscopy}

CDspectra of BfEAS_F58L and BfEAS_9AS-deletion were measured to analyze the native protein folding of the variants. The spectra were recorded in a range of $260 \mathrm{~nm}$ to $190 \mathrm{~nm}$.

A

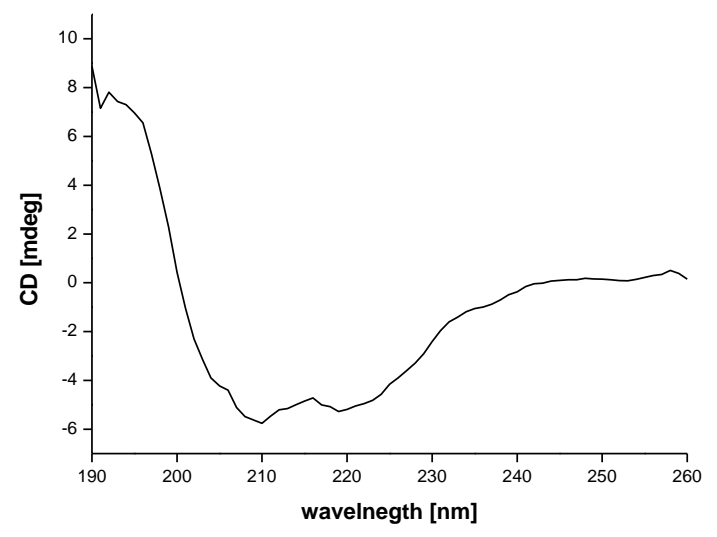

C

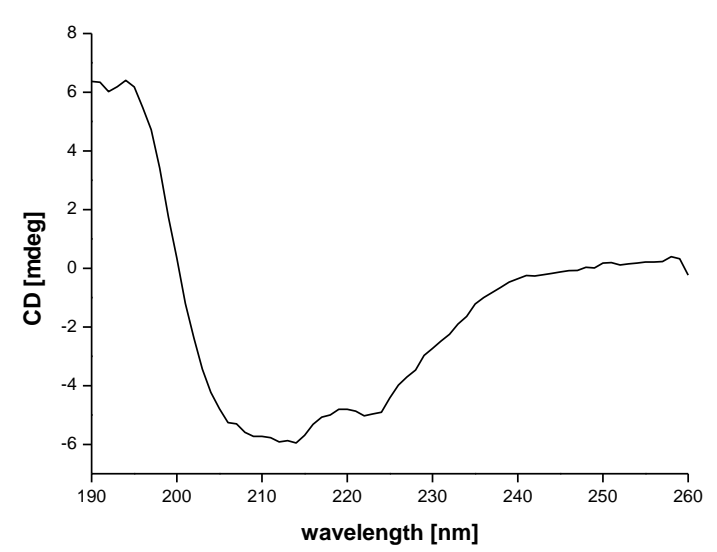

B

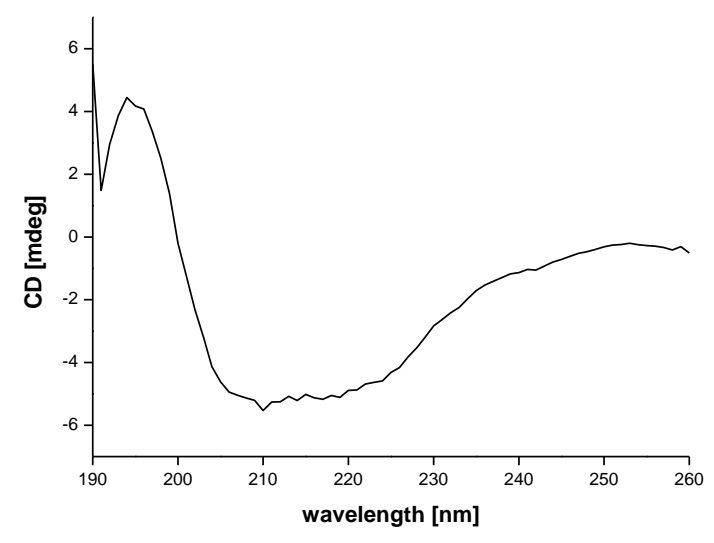

Figure 3.45: CD sepcra of purified BfEAS_F58L (A) and BfEAS_9AS-deletion (B) in comparison to BfEAS wild type (C). Spectra were measured in50 mM sodium phosphate buffer, $\mathrm{pH} 8.0$ on a Chirascan ${ }^{\mathrm{TM}} \mathrm{CD}$ Spectrometer (Applied Photophysics Ltd., Leatherhead, United Kingdom) in the range of $260 \mathrm{~nm}$ to $190 \mathrm{~nm}$. The figure shows the spectra of one experiment.

In Figure 3.45 CD spectra of the variants in comparison to wild type (C) are shown. Like the wild type the variants had two negative peaks and one positive peak which are characteristic for proteins consisting of $\alpha$-helix (Panagakou et al., 2012). The negative peaks of BfEAS_F58L (A) were at $210 \mathrm{~nm}$ and $219 \mathrm{~nm}$ and the positive peak at $192 \mathrm{~nm}$, whereas the peaks of BfEAS_9AS-deletion (B) were at $210 \mathrm{~nm}, 222 \mathrm{~nm}$ and $193 \mathrm{~nm}$. 


\subsubsection{Analysis of products}

\subsection{Radio-HPLC}

Analysis of products derived from reactions of BfEAS variants with $\left[1-{ }^{14} \mathrm{C}\right]$-labeled substrates were carried out by radio-HPLC. The reactions were incubated and extracted as described in 2.3.13.2. The conversion of 9 $\alpha$-HPOTby the variants BfEAS_F58L and BfEAS_9AS-deletion in comparison to the wild type areexemplarily shown in Figure 3.46. The reaction of BfEAS_F58L leads to a decrease in epoxy alcohol formation and in a slight increase of 9-oxo nonanoic acid. Colnelenic acid was not detected. The products of BfEAS_9AS-deletion variant were similar to the products of the wild type, with the exception that epoxy alcohol slightly decreased and only small amounts of divinyl ether CnA were detected. The peak at about 11 min could not be identified.

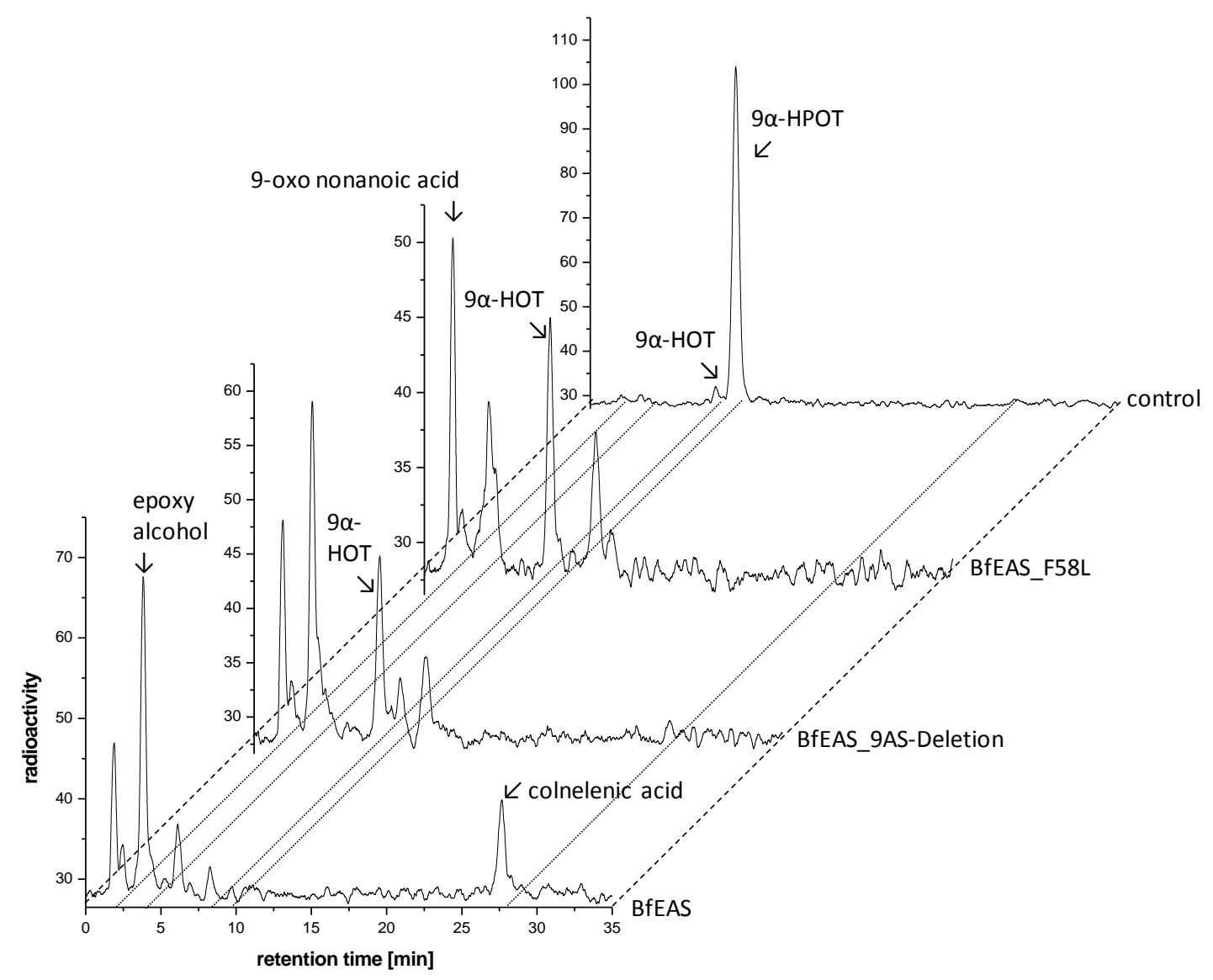

Figure 3.46: Product analysis from incubation of BfEAS, BfEAS_F58L, and BfEAS_9AS-deletion with $\left[1-{ }^{14} \mathrm{C}\right]-9 \alpha-$ HPOT. The purified enzymes were incubated with the radio-labeled substrate for $30 \mathrm{~min}$ at RT. After extraction with diethyl ether the products were analyzed by RP-HPLC coupled to a radio-detector. The figure is representative for two to five experiments.

Incubation with 9-HPOD showed that with the BfEAS_F58L variant the epoxy alcohol was strongly decreased whereas the HPL product was strongly increased (see appendix Figure 9.4 A). By incubation with 9-HPOD the 9AS-deletion variant was not as active as the wild type or other variants. 
InFigure 3.47 the chromatograms for conversion of $13 \alpha$-HPOT by BfEAS variants are exemplarily shown. Similar to the wild type the variants did not generate any divinyl ether derivative. BfEAS_F58L variant showed a strong reduction in formation of epoxy alcohol and a slight increase in formation of oxo fatty acid. BfEAS_9AS-deletion variant showed also a decrease in epoxy alcohol formation as well as a slightly increase in oxo fatty acid formation.

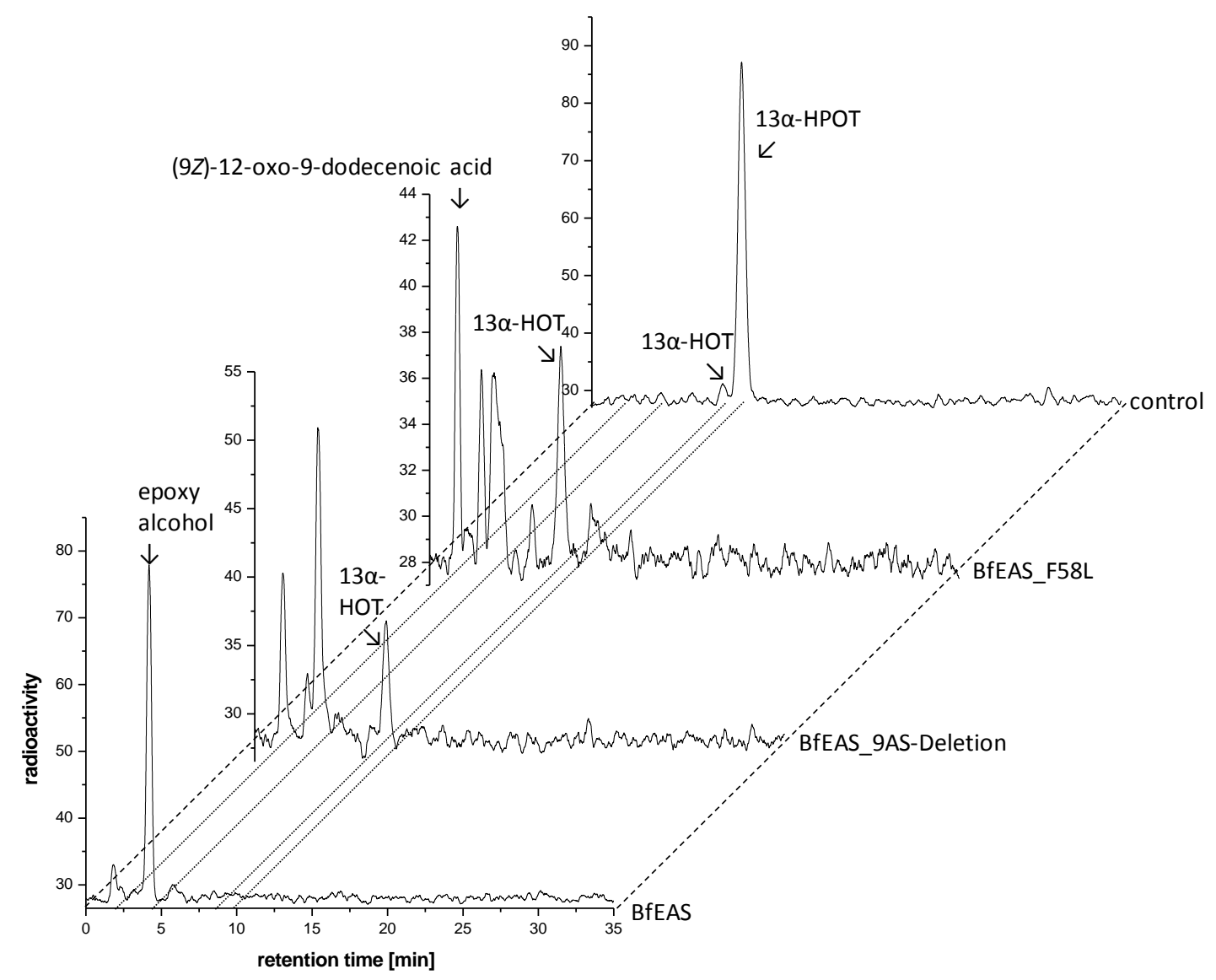

Figure 3.47: Product analysis from incubation of BfEAS, BfEAS_F58L, and BfEAS_9AS-deletion with [1- $\left.{ }^{14} \mathrm{C}\right]-13 \alpha-$ HPOT. The purified enzymes were incubated with the radio-labeled substrate for 30 min at RT. After extraction with diethyl ether the products were analyzed by RP-HPLC coupled to a radio-detector.The figure is representative for two to five experiments.

Similarly, conversion of 13-HPOD by both variants (BfEAS_F58L and BfEAS_9AS-deletion) showed strong decreases of EASproducts. BfEAS_F58L showed a slight increase of HPLproducts, whereas BfEAS_9AS has HPLproduct amounts like the wild type. The F58L variant was more active than the 9AS-deletion variant. Similar to the wild type DES products were not generated (see appendix Figure 9.4B).

In Table 3.8product analysis of BfEAS wild type and various BfEAS variants with radio-labeled 9- and 13-hydroperoxides are summarized. 
Table 3.8: Product specificities of BfEAS wild type and various BfEAS variants. Affinity purified enzymes were incubated with [1- ${ }^{14} \mathrm{C}$-labeled hydroperoxy fatty acids for approx. $30 \mathrm{~min}$ at RT. After extraction with diethyl ether the isolated products were analyzed by RP-HPLCthat was coupled to a radio-detector and quantified by integration of the respective peak area. The relative amounts of each product is indicated by the number of "+".

\begin{tabular}{|c|c|c|c|c|c|}
\hline substrate & variant & $\begin{array}{c}\text { w-oxo fatty acids } \\
\text { (= HPL activity) }\end{array}$ & $\begin{array}{l}\text { epoxy alcohols } \\
\text { (= EAS activity) }\end{array}$ & $\begin{array}{c}\text { divinyl ether } \\
\text { (= DES activity) }\end{array}$ & other products \\
\hline \multirow[t]{9}{*}{ 9-HPODE } & $W t$ & + & ++++ & + & n. d. \\
\hline & F58L & +++ & + & n. d. & + \\
\hline & F60L & + & ++++ & - & - \\
\hline & P75A & +++ & + & n. d. & + \\
\hline & P75S & + & + & n. d. & - \\
\hline & F223I & ++ & ++++ & n. d. & - \\
\hline & F223L & + & +++++ & - & - \\
\hline & $\mathrm{N} 224 \mathrm{Q}$ & + & ++++ & + & n. d. \\
\hline & 9AS-deletion & + & ++++ & - & n. d. \\
\hline \multirow[t]{9}{*}{$9 \alpha$-НРОТ } & Wt & ++ & +++ & ++ & + \\
\hline & F58L & ++ & + & n. d. & + \\
\hline & F60L & ++ & ++++ & - & - \\
\hline & P75A & ++ & + & n. d. & + \\
\hline & P75S & ++ & + & n. d. & + \\
\hline & F223I & ++ & ++++ & - & - \\
\hline & F223L & ++ & ++++ & - & - \\
\hline & N224Q & ++ & +++ & + & + \\
\hline & 9AS-deletion & ++ & ++ & - & + \\
\hline \multirow[t]{9}{*}{ 13-HPOD } & $W t$ & + & ++++ & n. d. & n. d. \\
\hline & F58L & ++ & + & n. d. & + \\
\hline & F60L & ++ & ++++ & n. d. & n. d. \\
\hline & P75A & ++ & + & n. d. & + \\
\hline & P75S & + & + & n. d. & n. d. \\
\hline & F223I & + & + & n. d. & - \\
\hline & F223L & + & +++ & n. d. & n. d. \\
\hline & $\mathrm{N} 224 \mathrm{Q}$ & + & +++ & n. d. & - \\
\hline & 9AS-deletion & + & + & n. d. & n. d. \\
\hline \multirow[t]{9}{*}{$13 \alpha$-НРОТ } & $W t$ & + & +++++ & n. d. & n. d. \\
\hline & F58L & ++ & + & n. d. & + \\
\hline & F60L & ++ & +++++ & n. d. & n. d. \\
\hline & P75A & ++ & + & n. d. & + \\
\hline & P75S & + & + & n. d. & - \\
\hline & F223I & ++ & + & n. d. & + \\
\hline & $\mathrm{F} 223 \mathrm{~L}$ & + & +++++ & n. d. & n. d. \\
\hline & N224Q & + & +++ & n. d. & + \\
\hline & 9AS-deletion & + & +++ & n. d. & n. d. \\
\hline
\end{tabular}




\subsection{RP-HPLC/MS}

Product formations of the various BfEAS variants were also analyzed by RP-HPLC/MS'. Reactions were performed as described (2.3.13.2 and 2.3.13.3). After purification the variants were incubated with 9- and 13-hydroperoxides derived from LA or $\alpha$-LeA.

The extracted ion chromatogram and associated tandem mass spectrum and potential fragment pattern for the conversion of $9 \alpha$-HPOT by BfEAS_F58L is shown in Figure 3.48. The insertion in A shows the extracted ion chromatogram of BfEAS wild type. The fragment pattern identified the epoxy alcohol as (12Z,15Z)-9-hydroxy-10,11-epoxy-12,15-octadecadienoic acid (Figure 3.48 B).
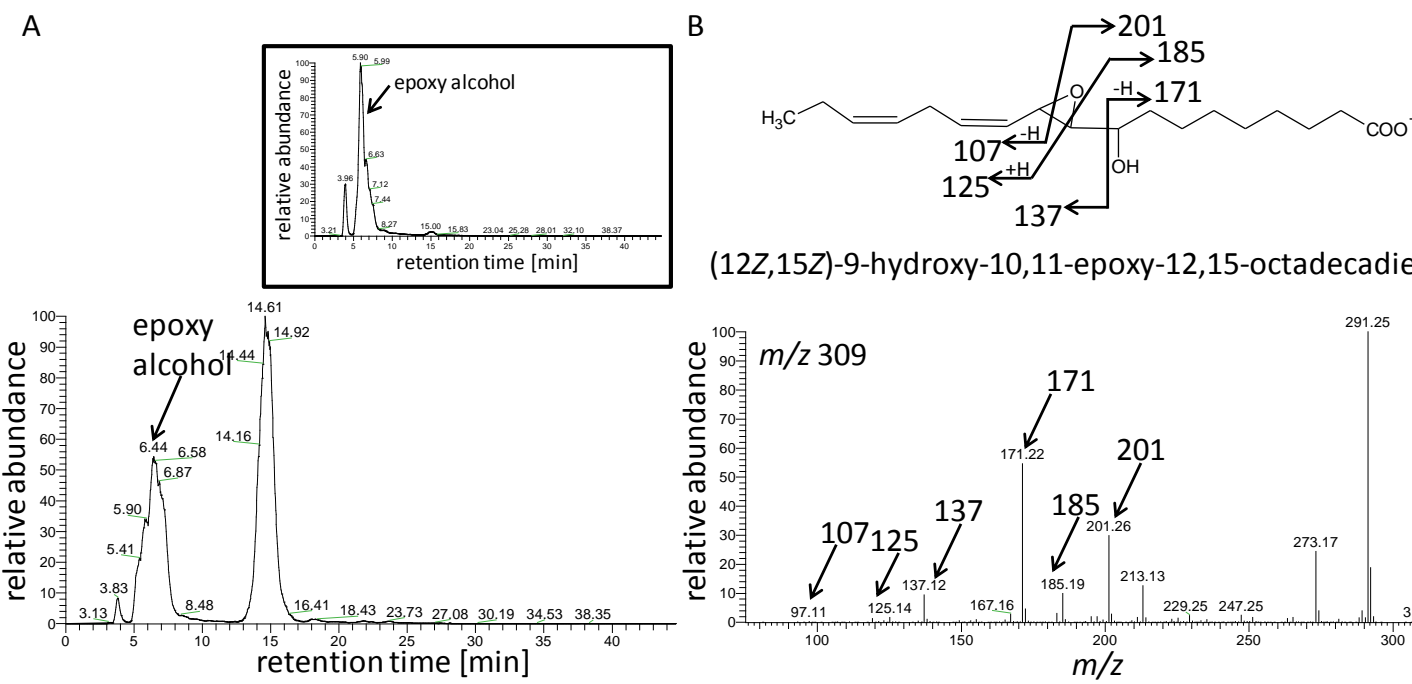

(12Z,15Z)-9-hydroxy-10,11-epoxy-12,15-octadecadienoic acid

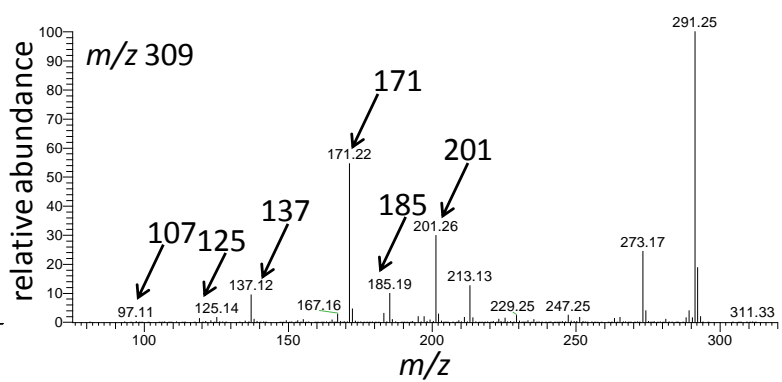

Figure 3.48: RP-HPLC/MS ${ }^{2}$-analysis of products formed by incubation of BfEAS_F58L with $9 \alpha$-HPOT in comparison to the wild type (insertion). Shown are the extracted ion chromatograms $(\mathrm{m} / z$ 309) (A)and the associated tandem mass spectrum (B).

The extracted ion chromatograms and associated tandem mass spectra and potential fragment patterns of BfEAS_9AS-deletion were similar to that of BfEAS_F58L (data not shown). BfEAS_9ASdeletion variant was not active as the other two variants, because less amounts of epoxy alcohol were detected.

Figure 3.49 shows the extracted ion chromatogram and the associated tandem mass spectrum and potential fragment patterns of the conversion of BfEAS variant F58L with $13 \alpha$-HPOT in comparison to the wild type. The epoxy alcohol (9Z,15Z)-11-hydroxy-12,13-epoxy-9,15-octadecadienoic acid (Figure 3.49B) was detected. 
A

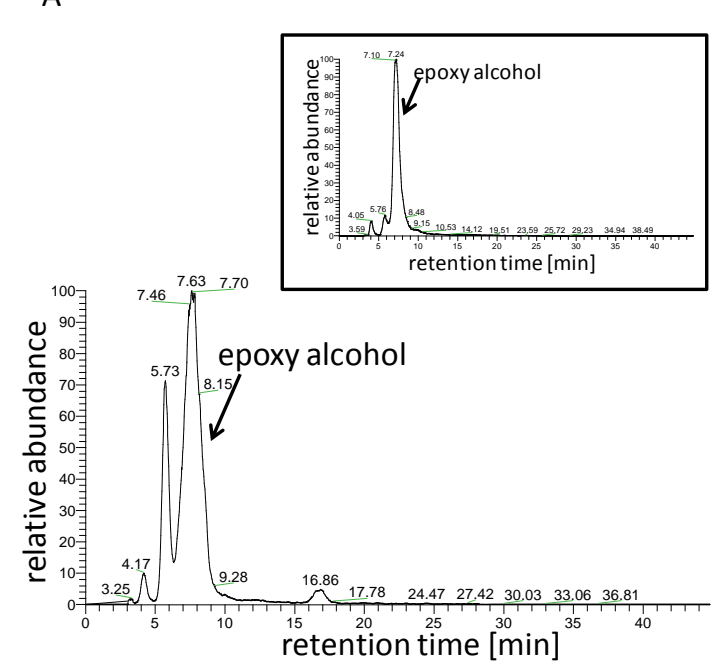

B

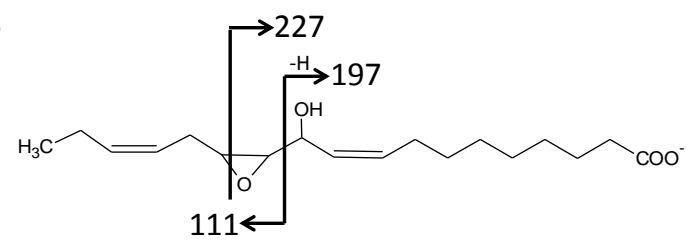

(9Z,15Z)-11-hydroxy-12,13-epoxy-9,15-octadecadienoic acid

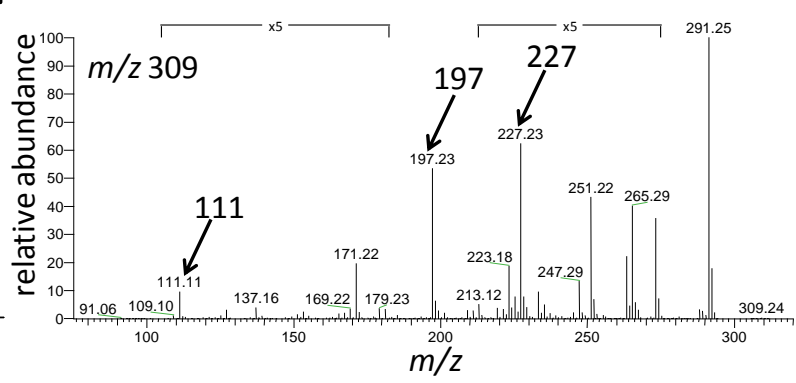

Figure 3.49: RP-HPLC/MS ${ }^{2}$-analysis of products formed by incubation of BfEAS_F58L with $13 \alpha-\mathrm{HPOT}$ in comparison to the wild type (insertion). Shown are the extracted ion chromatogram $(\mathrm{m} / \mathrm{z} 309)(A)$ and the associated tandem mass spectrum (B).

The RP-HPLC/MS ${ }^{2}$ analysis of conversions of other BfEAS variants with 9- and 13-hydroperoxides derived from LA and $\alpha$-LeA verified also the formation of epoxy alcohols (data not shown). 


\section{Discussion}

The aim of the study was the biochemical characterization of the CYP74-enzymes in the moss Physcomitrella patens and in the lancelet Branchiostoma floridae. In the first part the biochemical properties of PpAOS1 were analyzed and compared with that of previously characterized PpAOS2 (diploma thesis Scholz, 2009) and PpHPL (Stumpe et al., 2006a).

For this purpose a purification protocol was established that enabled the generation of high amounts of homogenous protein. In addition previously established methods for the isolation of PpAOS2 and PpHPL were modified in order to generate high amounts of homogenous protein. With purified PpAOS1 different biochemical analysis were performed: the specificity of PpAOS1 against hydroperoxides derived from $C_{18}$ fatty acids $L A, \alpha$-LeA and $\gamma$-LeA and the $C_{20}$ fatty acid ARA were determined. The products from those reactions were analyzed by radio-HPLC and RP-HPLC/MS ${ }^{2}$. Based on bioinformatic data different amino acids possibly involved in catalysis were mutagenized by site-directed mutagenesis and the respective enzyme variants were analyzed with respect to their biochemical properties and compared to those of the wild type PpAOS1, PpAOS2 and PpHPL. In the second part the biochemical parameters of the fourth CYP74-enzyme EAS from B. floridae were determined to compare it with AOS, DES and HPL from plants.

\subsection{Biochemical characterization of CYP74-enzymes}

\subsubsection{Spectroscopic analysis (UV/vis and CD)}

In plants CYP74-enzymes are targeted to different membranes of the plastid envelope (Froehlich et al., 2001) where they bind to the hydrophobic bilayer via a patch of hydrophobic helices (Schaller and Stintzi, 2009). As pointed out recently by Schückel and coworkers, the hydrophobic nature of these enzymes renders the expression and solubilisation difficult (Schückel et al., 2012). In order to improve the solubility of PpHPL an amino acid sequence (MAKKTSS) was added at the N-terminus, directly behind the hexahistidine-tag. A similar approach was successfully used by Lee and coworkers before for the purification and crystallization of AtAOS (Lee et al., 2008). Initially, purification of PpHPL and the AOS isoforms PpAOS1 and PpAOS2 was performed according to previously established protocols (diploma thesis Scholz, 2009). As these procedures only led to samples containing variable amounts of contaminating proteins (see Figure 3.1 and Figure $3.3 \mathrm{~A}$ ) the purification protocol was optimized. To generate more homogenous protein by IMAC-purification the stepwise imidazole gradient (5\% and $100 \%$ ) was changed into a linear imidazole gradient (5\% to $100 \%$ in $20 \mathrm{~min}$ ), where the unspecifically bound proteins were eluted from the column. This 
modification led to more homogenous protein (see Figure 3.2 and Figure 3.3 B and C). In some experiments a subsequent purification step by using size exclusion chromatography was used. However, as this procedure did not led to a significant improvement of the protein purity this procedure was only used for analyzing the native molecular weights of the proteins. The molecular weight of PpAOS1 and PpAOS2 as determined by SDS-PAGE analysis was approx. $55 \mathrm{kDa}$ and $57 \mathrm{kDa}$ for PpHPL. Size exclution chromatography determined for PpAOS1 a molecular weight of $40 \mathrm{kDa}$, for PpAOS2 of $240 \mathrm{kDa}$ and for PpHPL of $113 \mathrm{kDa}$. These findings indicated that PpAOS1 is a monomeric protein complex, while PpAOS2 may form at least under the applied experimental conditions a tetrameric or even pentameric complex. PpHPL on the other hand may exist as a homodimeric complex. Different oligomeric states have also been reported for other CYP74s: While for AOS of Arabidopsis (AtAOS) and for guayule (PaAOS) were found to be monomeres (Li et al., 2008, Hughes et al., 2006a), the HPL of pepper (CaHPL) was identified to form a trimeric protein complex (Shibata et al., 1995). Recently, it was shown that the oligomeric state of CYP74s might be influenced by different molecular factors as for example the presence or absence of detergents. Based on these different resulting effects new rules for CYP74-classification were proposed (Hughes et al., 2006b).

As mentioned above CYP74-enzymes belong to the P450-family and are thus heme containing enzymes. A prerequisite of these enzymes is that the heme cofactor is ligated by a cysteine as the fifth iron ligand on the proximal site and water as the sixth ligand at the distal site (Lee et al., 2008, Li et al., 2008). Consequently, the heme iron in all P450-enzymes (in this resting state) is low spin. In order to characterize the heme cofactor and its molecular micro-environment the purified proteins were analyzed by means of UV/vis-spectroscopy. Detected UV/vis spectra (see Figure 3.4) for the proteins showed the characteristic main absorption maximum at about $410 \mathrm{~nm}$ which is characteristic for hemeproteins and is known as the Soret-band. Importantly, all spectra lacked an absorption maximum at $630 \mathrm{~nm}$ which is characteristic for high-spin iron-III-complexes. Therefore the UV/vis analysis of PpAOS1, PpAOS2 and PpHPL indicates that all three proteins contain low-spin hemeiron complexes. This finding is thus in line with the spin-states reported for all CYP74s so far and is characteristic for P450-enzymes (Noordermeer et al., 2001b).

UV/vis analysis also indicated that PpAOS1 and PpAOS2 had significant differences in their heme contents. While PpAOS1 has a content of approx. $30 \%$ the content of PpAOS2 is only $4 \%$. The heme content of PpHPL is higher (46\%) than of both AOS isoforms.

The analysis of protein folding by CD spectroscopy (see Figure 3.5 and Figure 3.6) showed signals typical for proteins with a mainly $\alpha$-helical fold (Panagakou et al., 2012). Thus this finding goes in line with the fact that both characterized AOS-structures are mainly composed of $\alpha$-helices (Lee et al., 2008, Li et al., 2008). 


\subsubsection{Analysis of products (radio-HPLC and RP-HPLC/MS ${ }^{2}$ )}

To analyze the different products that were formed by incubations of various CYP74-enzymes (e. $\mathrm{g}$. PpAOS1, PpAOS2 and PpHPL) with different 9-and 13-hydroperoxides derived from LA and $\alpha$-LeA, radio-HPLC and RP-HPLC/MS ${ }^{2}$ analysis were performed.

Analysis of products formed by conversions of radio-labeled 9-hydroperoxides with PpAOS1 indicated that mainly typical AOS products, the $\alpha$ - and $y$-ketols were formed (Figure 3.7andFigure 9.1 A). It is well established that these metabolites are formed non-enzymatically by hydrolysis of the allene oxide. While the $\alpha$-ketol represents the main product of this reaction, the $\gamma$-ketols are normally formed in lower amounts. Interestingly, in addition to these characteristic AOS products the HPL-specific $\omega$-oxo fatty acid product was also detected as side product. The occurrence of the characteristic HPL product ( $\omega$-oxo fatty acid, (Vick and Zimmerman, 1976)) beside the $\alpha$ - and $\psi$-ketols (Hamberg, 1987, Brash et al., 1988) suggests that PpAOS possess an inherent HPL activity. This finding is in line with previous studies on rice AOS and Arabidopsis AOS (Cho et al., 2011).Conversions of radio-labeled 13-hydroperoxides with PpAOS1 gave similar results (Figure 3.8and Figure 9.3 B). However, in addition to the ketols and oxo fatty acid a cyclopentenone derivative wasformed, which arose from the non-enzymatical cyclization of the intermediary formed allene oxide(Hamberg et al., 1988).

On the other hand, incubations of PpHPL with 9-hydroperoxides derived from radio-labeled LA and $\alpha$ LeA led not only to the formation of 9-oxo nonanoic acid as main product but yielded $\alpha$ - and $y$-ketols (Figure 3.7 andFigure 9.1 A). Conversions of radio-labeled 13-hydroperoxides gave similar results (Figure 3.8 and Figure 9.1 B).Thus results indicate that also PpHPL exhibits inherent AOSactivity. It should be noted at this point, that the $\alpha$-ketols were formed in lower amounts compared to the $\gamma$ ketols that were formed in higher amounts. This finding was surprising since as mentioned above the $\alpha$-ketol is normally formed in high amounts and the $\gamma$-ketol in significantly less amounts (Grechkin et al., 1991). The favored formation of the $\gamma$-ketol may have occurred because of the acidic $\mathrm{pH}$ used in the applied buffer systems. As reported by Grechkin and coworkers the trajectory of this unspecific reaction is highly $\mathrm{pH}$-dependent; in particular formation of the $\mathrm{\gamma}$-ketol is favoured at low (acidic) $\mathrm{pH}$ values (Grechkin et al., 1991). These conditions were used for the product analysis experiments. The existence of both enzymatic activities suggests that the catalytic pathways of AOS and HPL are close interconnected as has been suggested before (Lee et al., 2008, Brash, 2009,Cho et al., 2011). To analyze if this close interconnection of the catalytic pathways also applies to other members of the CYP74-family conversions of StDES and AsDES with radio-labeled 9-and 13-hydroperoxides derived from $\alpha$-LeA were performed. The radio-HPLC analysis showed that beside the main DESproduct (divinyl ether) also some HPLproduct ( $\omega$-oxo fatty acid) can be detected (see Figure 3.9). These results led to the hypothesis that the catalytic pathways of DES and HPL were also close 
interconnected. Unfortunately the handling of both DESisozymes turned out to be difficult, since the protein yield after purification was considerably low. Thus we did not follow up the mechanistic details of these reactions.

The products formed by conversion of $13 \alpha$-HPOT by PpAOS1 and PpHPL were verified by RPHPLC/MS ${ }^{2}$ (see Figure 3.10toFigure 3.13).

\subsubsection{Kinetic analysis (pH optimum and kinetic parameters)}

For determination of the $\mathrm{pH}$ optimum the conversion of 13-HPOD to the corresponding products was measured by recording the decrease at $234 \mathrm{~nm}$ in various buffers. This method gives information about the initial velocity in dependence of various $\mathrm{pH}$ reaction buffers. Initial velocity can be influenced by enzyme concentration and substrate concentration. Therefore the concentrations were kept constant at the measurements.

PpAOS1 showed highest activity at pH 6.5 (see Figure 3.14). A similar pH optimum (pH 6.0) was recently reported for PpAOS1 (Bandara et al., 2009). In contrast to the slightly acidic pH of PpAOS1, PpAOS2 has a neutral pH optimum at pH 7.0 (diploma thesis Scholz, 2009).

The slightly acidic $\mathrm{pH}$ optimum of PpAOS1 and the neutral $\mathrm{pH}$ optimum of PpAOS2 are consistent to $\mathrm{pH}$ optimum of the corresponding PpLOX isoforms (Anterola et al., 2009). This is important since the fatty acid hydroperoxide substrates for AOS activity are provided by LOXs (Wasternack and Kombrink, 2010).

Interestingly, the $\mathrm{pH}$-values optimal for catalytic activity of both AOS isoforms are also in line with their sub-cellular localizations. PpAOS2 is localized in the plastid, where, due to the increasing concentration of $\mathrm{H}^{+}$during photosynthesis, the milieu might become slightly acidic (Taiz, 2007). PpAOS1 on the other hand is localized in the cytosol at a nearly neutral pH (Scholz et al., 2012).

Similar pH optima have also been reported for different AOSs from flowering plants that are between pH 7.0 and pH 8.0 (Bandara et al., 2009).

P. patens contain $C_{18}$ fatty acids LA and LeA and $C_{20}$ fatty acids ARA and EPA (Dembitsky, 1993, Girke et al., 1998). As these fatty acids can be used as substrates for oxylipin biosynthesis two different biosynthetic pathways have been proposed: the octadecanoid and the eicosanoid pathway (Wichard et al., 2005). Thus it was of interest to assign which PpAOS isoform may be involved in which pathway. For this purpose the substrate specificity was determined by analyzing the kinetic parameters $K_{M}$ and $V_{\max }$ of PpAOS1 for the different substrates and comparing those results to the data obtained for PpAOS2 which were part of a former study (diploma thesis Scholz, 2009). In order to compare the kinetic parameters of both AOSisoforms it was essential to take the unequal heme occupancy of both proteins into account. Thus, the data for PpAOS2 were re-calculated by 
considering the respective heme occupancy mentioned above. The data obtained for PpAOS1 were analogously modified.

The kinetic analysis showed that PpAOS1 can use both the 9- and 13-hydroperoxides derived from $C_{18}$ fatty acids LA, $\alpha$-LeA and $\gamma$-LeA and 12 -HPETE derived from $C_{20}$ fatty acid ARA as substrates with a similar activity (see Table 3.3) indicating that PpAOS1 might be involved in the octadecanoid as well as in the eicosanoid pathway. The $\mathrm{k}_{\text {cat }}$-values of PpAOS1 are comparable with those reported for other AOS (Hughes et al., 2006a, Lee et al., 2008).

A re-evaluation of the data derived from the kinetic analysis of the reactions catalyzed by PpAOS2 (Table 4.1) showed that $\mathrm{C}_{18}$ fatty acid derived hydroperoxides are only very poor substrates, whereas $\mathrm{C}_{20}$ fatty acid derived 12-HPETE are converted with a similar high activity as it was shown for PpAOS1.

Table 4.1:Re-evaluation of the kinetic parameters for the reaction of PpAOS2 with different hydroperoxides derived from $\mathrm{C}_{18}$ - and $\mathrm{C}_{20}$ fatty acids. The kinetic parameters were measured at a photometer, where the initial time-dependent substrate consumption at $234 \mathrm{~nm}$ with different substrate concentrations was determined. For the analysis between 20 and 30 data points were fitted to the Michaelis-Menten equation. The concentration of PpAOS2 was $100 \mathrm{nM}$ except for the measurements of 12 -HPETE $(1 \mathrm{nM})$. The values for $k_{\text {cat }}$ were calculated by considering heme occupancy of approx. $4 \%$.

\begin{tabular}{|c|c|c|c|c|}
\hline substrate & $\begin{array}{c}\mathrm{K}_{\mathrm{M}} \\
{[\mu \mathrm{M}]}\end{array}$ & $\begin{array}{c}\mathrm{V}_{\max } \\
{[\mu \mathrm{M} / \min ]}\end{array}$ & $\begin{array}{c}\mathbf{k}_{\mathrm{cat}} \\
{[1 / \mathrm{min}]}\end{array}$ & $\begin{array}{c}k_{\mathrm{cat}} / K_{\mathrm{M}} \\
{\left[\mathrm{min}^{-1} \mathrm{M}^{-1} \times 10^{6}\right]}\end{array}$ \\
\hline 9-HPOD & $36+/-5$ & $0.02+/-0.001$ & 5 & 0.14 \\
\hline $9 \alpha-$ НРОТ & $40+/-4$ & $0.01+/-0.001$ & 2.5 & 0.06 \\
\hline $9 \gamma$-НРОT & $28+/-4$ & $0.03+/-0.002$ & 7.5 & 0.27 \\
\hline 13-HPOD & $27+/-3$ & $0.04+/-0.002$ & 10 & 0.37 \\
\hline $13 \alpha-\mathrm{HPOT}$ & $30+/-5$ & $0.02+/-0.002$ & 5 & 0.17 \\
\hline 13ץ-HРOT & $42+/-10$ & $0.02+/-0.002$ & 5 & 0.12 \\
\hline 12-HPETE & $10+/-5$ & $0.49+/-0.057$ & 12250 & 1228.69 \\
\hline
\end{tabular}

This result indicates that PpAOS2 exhibits in contrast to PpAOS1 a distinct specificity for $\mathrm{C}_{20}$-derived hydroperoxy derivatives and thus suggests a distinct involvement in the eicosanoid pathway (Wichard et al., 2005).

The data obtained from the kinetic measurements suggests that the substrate preference of PpAOS1 is characteristic for unspecific 9/13-AOS. The majorities of biochemically characterized AOS found in the literature are substrate specific 13-AOS and are thus members of the subfamily CYP74A. Unspecific 9/13-AOS as well as 9-AOS, 9-HPL and 9/13-HPL are part of the CYP74C-family. HPLs and DES with a preference for 13-hydroperoxides belong to the CYP74B-family and DES with a preference for 9-hydroperoxides to CYP74D (Stumpe and Feussner, 2006, Gogolev et al., 2011). In comparison to both AOS isoforms for PpHPL was reported that it can accept $C_{18^{-}}$as well as $C_{20^{-}}$-derived hydroperoxides as substrates (Stumpe et al., 2006a) and that it prefers 9-hydroperoxides derived 
from $\mathrm{C}_{18}$ fatty acids. The phylogenetic analysis in Figure 1.3shows that the division in the different subfamily groups applies to CYP74s from higher plants but not for CYP74-enzymes AOS1, AOS2 and HPL from non-flowering plants like the moss Physcomitrella.

\subsubsection{Mutagenesis}

For AOS from Arabidopsis it is known that the catalytic activity can be altered by mutating specific determinants that connect the AOS and HPL activities (Lee et al., 2008, Cho et al., 2011). However, the AOS used in these experiments has a distinct specificity for 13-hydroperoxides as substrates (Hughes et al., 2006a). Thus it was of interest to investigate whether the respective amino acids are also triggering the catalytic activity of unspecific 9-/13-AOS. For this purpose in the present work sitedirected mutagenesis of these particular determinants was performed on PpHPL and PpAOS1 which have preferences for 9-and 13-hydroperoxides as substrates (Stumpe et al., 2006a). It was hypothesized that a conserved $\mathrm{F}$ is an essential residue in the active site of AOS and plays a central role in the catalysis by stabilizing an intermediary formed carbon centered substrate radical. The replacement of the F by L, converts the AOS into a HPL (Lee et al., 2008, Cho et al., 2011). In order to analyze if this concept can also be applied on AOS with a broad substrate range, the respective PpAOS1 variant (PpAOS1_F93L) was generated. In HPL at this particular position is a conserved L located. However, PpHPL contains at this position the F which is characteristic for AOS-enzymes. In order to analyze the function of this amino acid on HPL-catalysis the PpHPL_F151L variant was generated. Additionally PpHPL_A169S was generated, in which a HPL-specific A was substituted by an AOS-specific S.

As reported for tomato AOS (LeAOS3) at position 295 is a further F which is strictly conserved in all known AOS and HPLs and can alter enzyme activity by substitution (Toporkova et al., 2008). PpHPL has at this particular position a conserved L instead of the F. Thus PpHPL_F336L variant was generated to analyze the function of this residue in HPL-catalysis. It was also reported for LeAOS3 that it has AOS- and HPLactivities by substitution of lysine (K) by S (LeAOS3_K302S) (Toporkova et al., 2010). PpAOS1 has $S$ and PpHPL a $C$ at this position. These residues were substituted by $K$ (PpAOS1_S287K and PpHPL_C343K).

Beside the F (AtAOS_F320, LeAOS3_F295) is a conserved N which substitution by a Q should abolish enzyme activity (Lee et al., 2008). Therefore PpAOS1_N281Q and PpHPL_N337Q were generated.

With the generated variants of PpAOS1 and PpHPL spectroscopic analysis and product analysis were performed. 


\subsubsection{Spectroscopic analysis (UV/vis and CD)}

UV/vis spectra of PpHPL variants showed no significant changes (see Figure 3.19). They looked similar to the spectra of the wild type enzyme. Like the wild typeenzyme the different HPL variants appeared to contain low-spin-heme iron as it is characteristic for P450-enzymes (Noordermeer et al., 2001b). The heme content of PpHPL_F151L (45\%) is very similar to that of PpHPL wild type (46\%). PpHPL_A169S (31 \%) and PpHPL_F151L, A169S (37 \%) has light decreases in the heme content. CD spectra showed that the variants had a similar protein folding like the wild type (see Figure 3.20). As the $C D$ spectrum of the wild type the $C D$ spectra of the variants showed the typical peaks for proteins with a mainly $\alpha$-helical fold (Panagakou et al., 2012). Thus these results showed that sitedirected mutagenesis had no effects on protein folding.

\subsubsection{Analysis of products (radio-HPLC)}

To analyze the influence of the single amino acid exchanges on enzyme activity products formed by the different PpAOS1 and PpHPL variants were analyzed by radio-HPLC.

Incubations of the PpAOS1 variant in which the respective $F$ was substituted by L (PpAOS1_F93L) showed that the product activity was altered from AOS- to HPLactivity paralleling the results obtained for substrate specific AtAOS. This effect on the activity was also observed for incubations with all 9- and 13-hydroperoxides as substrates (see Figure 3.21, Figure 3.22andFigure 9.2).This has before only been reported for AtAOS which has a preference for 13-hydroperoxides (Lee et al., 2008, Cho et al., 2011,Hughes et al., 2006a). Thus, the mutational studies of PpAOS1 of this work showed for the first time, that the AOS activity of an unspecific enzyme can also be converted to HPLactivity. It may be considered that site-directed mutagenesis of the AOS-specific F in PpHPL would increase the HPLactivity and decrease the inherent AOSactivity that was found in wild type. RadioHPLCanalysis showed that the product pattern of the variant, where the conserved AOS-specific F was substituted by HPL-specific L, was similar to that of wild type. Lee as well as Schaller have proposed reaction mechanisms for AOS and HPL with a common radical intermediate (epoxy allylic radical) and that product formation takes place by the property of stabilization of the radical by AOSspecific F or not by HPL-specific L (Lee et al., 2008, Schaller and Stintzi, 2009). Brash also proposed that the enzymes have common intermediates and that they can be synthesized either through ionic or radical routes and that the unstable hemiacetal is possible through both routes (Brash, 2009). In other HPLs this proposed mechanisms may be possible. In case of HPL of Physcomitrella it could be that the occurrence of the AOS-specific $F$ in the PpHPL-sequence instead of HPL-specific $L$ is responsible for the inherent AOS activity. For HPL activity may other determinants be responsible.

Substitution of HPL-specific A by AOS-specific S (PpHPL_A169S) showed also similar product pattern as the wild type. Also the double variant (PpHPL_F151L, A169S) did not show any alteration in its 
product pattern. These results suggest that determinants essential for AOS-catalysis are not essential for the activity in PpHPL. Therefore in PpHPL other determinants seem to be relevant for enzyme specificity. These findings are similar to those reported before for HPL of tomato (Lee et al., 2008), where the analogous double variant LeHPL_L101F, A119S exhibited only $5 \%$ AOS activity.

Generation of PpAOS1_S111A, PpAOS1_S287K, PpHPL_L336F and PpHPL_C343K were not successful. Additionally to the site-directed mutagenesis analysis of AOS and HPL a set of different StDES variants were generated for product analysis. The problem was that the expression of the mutants was too low to perform product analysis. Thus expression conditions have to be improved. 


\subsection{Biochemical characterization of BfEAS}

Recently EAS was beside AOS, DES and HPL reported as fourth member of the CYP74-enzymes (Lee et al., 2008). Until now the enzymes of EAS pathway were not detected in plants, only EAS products have been found in potato and rice (Hamberg, 1999, Feussner and Wasternack, 2002,Ohta et al., 1990).However, EAS activity was very recently also observed in the rice pathogen Magnaporthe salvinii (Wennman and Oliw, 2012). Along these lines it has been discussed that EAS seems to be involved in pathogen defense (Feussner and Wasternack, 2002, Blée, 1998b). Therefore it is of interest to characterize the biochemical properties of this enzyme and to compare it with other CYP74-enzymes which have been found in plants. In this study EAS of the lancelet B. floridae was purified and used for kinetic and spectroscopic analysis. Product analysis was performed by radioHPLC and RP-HPLC/MS². For further analysis site-directed mutagenesis were carried out.

\subsubsection{Spectroscopic analysis (UV/vis and CD)}

First a purification protocol was established that enabled the isolation of highly homogenous protein (see Figure 3.24). Analysis by size exclution chromatography determined a native molecular weight of $60 \mathrm{kDa}$ for BfEAS. Therefore BfEAS is like PpAOS1 a monomeric protein.

With the purified enzyme further analysis were performed. Absorption profiles obtained by UV/vis analysis and CD analysis of BfEAS were similar to the analysis of PpAOS and PpHPL. The UV/vis spectrum confirmed the presence of the heme cofactor in BfEAS, because the spectrum showed characteristic absorption maximum at $420 \mathrm{~nm}$. As the two AOS and the HPL of $P$. patens the EAS of B. floridae also seem to contain a low-spin heme-iron-III-complex, because high-spin-iron-III-complex characteristic maximum at $630 \mathrm{~nm}$ was not detected (see Figure 3.25).

The heme content of BfEAS is about $37 \%$ and almost similar to PpAOS1 (30\%) and PpHPL (46 \%).

The measured CD spectrum (see Figure 3.26) showed like PpAOS1 and PpHPL typical peaks for proteins with a mainly $\alpha$-helical fold (Panagakou et al., 2012) and thus suggests that EAS might exhibit a similar over-all structure as AtAOS (Lee et al., 2008).

\subsubsection{Analysis of products (radio-HPLC and RP-HPLC/MS²)}

Analyses of products formed by incubations of BfEAS with hydroperoxides were analyzed by radioHPLC and RP-HPLC/MS ${ }^{2}$.

Analysis of products by incubations with radio-labeled 9-and 13-hydroperoxides from LA and $\alpha$-LeA showed different product patterns by radio-HPLC analysis. Exemplarily with 9-hydroperoxide derived from $\alpha$-LeA as substrate the respective epoxyalcohol-derivative $(12 Z, 15 Z)-9$-hydroxy-10,11-epoxy12,15-octadecadienoic acid was detected as the main product (see Figure 3.28 and Figure 3.30). In 
addition formation of small amounts of the HPL product (9-oxo nonanoic acid) as well as the DESproduct (colnelenic acid) was detected (see Figure 3.28 and Figure 3.31). On the other hand incubations of BfEAS with 13-hydroperoxide derived from $\alpha$-LeA mainly led to formation of the epoxy alcohol derivative $(9 Z, 15 Z)-11$-hydroxy-12,13-epoxy-9,15-octadecadienoic acid as main product (see Figure 3.29 and Figure 3.32)and (9Z)-12-oxo-9-dodecenoic acid as the only side product (see Figure 3.29).

These findings suggest that conversions of 13-hydroperoxides from LA and $\alpha$-LeA were more specific than conversions of 9-hydroperoxides from LA and $\alpha$-LeA. The finding that during catalysis the characteristic products of EAS, DES and HPL arise reflects the close interconnection of the different enzymatic trajectories in catalysis of CYP74-enzymes and thus indicates that EAS, DES and HPL may have the same intermediate, an epoxy allylic radical, in catalysis (Brash, 2009).

Since the $\mathrm{C}_{18}$ fatty acids LA and $\alpha$-LeA are not the most abundant fatty acids in Branchiostoma it is questionable if the tested 9-and 13-hydroperoxides derived from LA and $\alpha$-LeA are the natural substrates of BfEAS. The $\mathrm{C}_{22}$ fatty acid DHA is one of the most abundant fatty acids in B. floridae (Lee et al., 2008). Therefore by incubations of DHA with different LOXsisoforms various hydroperoxide isomers can be generated and used as substrates for BfEAS. In this study the products formed from incubations DHA with different LOXisoforms from soybean (GmLOX1), moss (PpLOX2), Arabidopsis (AtLOX1) and potato (StLOX) were analyzed by RP-HPLC/MS ${ }^{2}$ to identify the possible hydroperoxide isomers that may be used for EAS analysis. Extracted ion chromatograms, associated tandem mass spectra and potential fragment patterns were consistent to known patterns (Hong et al., 2007). Conversion of DHA by GmLOX1 led to the formation of 17-hydroperoxide (see Figure 3.33), whereas conversions of DHA by AtLXO1 and StLOX led to the formation of 10-hydroperoxide (see Figure 3.35 and Figure 3.36), and by PpLOX the 14-hydroperoxide was generated (Figure 3.37).

After the identification of various hydroperoxide isomers derived from DHA, conversions of BfEAS with this hydroperoxides were analyzed and indeed incubations of BfEAS with hydroperoxides derived from $\mathrm{C}_{22}$ fatty acid led to the formation of the corresponding epoxy alcohols (see Figure 3.34and Figure 9.7 to Figure 9.9), where the hydroxygroup stays at its position and the epoxy group is at the adjacent double bond of the DHA-derived fatty acid hydroperoxide.

\subsubsection{Kinetic analysis ( $\mathrm{pH}$ optimum and kinetic parameters)}

The determined $\mathrm{pH}$ optimum of BfEAS is similar to that of other CYP74-enzymes. BfEAS showed the highest activity at pH 6.75 (see Figure 3.38). This optimum is between the optima of both AOS isoforms of P. patens (PpAOS1 pH 6.5 and PpAOS2 pH 7.0 (Scholz et al., 2012).

In B. floridae various fatty acids were the potential precursors of EAS substrates. As mentioned before $B$. floridae contains inter alia the $C_{18}$ fatty acids LA and $\alpha$-LeA as well as the $C_{22}$ fatty acid DHA. 
For determination of substrate preference the kinetic parameters $K_{M}$ and $V_{\max }$ of BfEAS for conversions of 13-hydroperoxides derived from the $\mathrm{C}_{18}$ fatty acids and 17-hydroperoxide derived from the $\mathrm{C}_{22}$ fatty acid were measured.

Kinetic analysis showed that BfEAS can use both 13-hydroperoxides derived from LA and $\alpha$-LeA (see Figure 3.39) as well as 17-HPDHA derived from DHA (see Figure 3.40) as substrates. The $\mathrm{k}_{\text {cat }}$-values were calculated by considering heme occupancy of $37 \%$ and showed that BfEAS has a preference for $13 \alpha$-HPOT as substrate. The $\mathrm{k}_{\mathrm{cat}} / \mathrm{K}_{\mathrm{M}}$-values of BfEAS for $13 \alpha$-HPOT were similar to that of PpAOS1.

The results of the first kinetic measurements and product analysis suggest that BfEAS can convert different hydroperoxy-substrates (9- and 13-hydroperoxides from LA and $\alpha$-LeA as well as 10-, 14and 17-hydroperoxides derived from DHA) to a large variety of different products. Products of incubations with 9- and 13-hydroperoxides are shown in Table 4.2.

Table 4.2: Products formed by conversions of 9 -and 13-hydroperoxides derived from $C_{18}$ fatty acids LA and $\alpha$ LeA by BfEAS.

\begin{tabular}{|c|c|c|c|c|}
\hline & 9-HPOD & 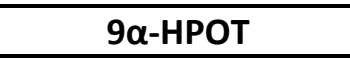 & 13-HPOD & $13 \alpha-\mathrm{HPOT}$ \\
\hline & (12Z)-9-hydroxy- & (12Z,15Z)-9-hydroxy- & (9Z,)-11-hydroxy- & (9Z,15Z)-11-hydroxy- \\
\hline $\begin{array}{l}\text { epoxy } \\
\text { alcohols }\end{array}$ & $\begin{array}{l}\text { 10,11-epoxy-12- } \\
\text { octadecenoic acid }\end{array}$ & $\begin{array}{l}\text { 10,11-epoxy-12,15- } \\
\text { octadecadienoic acid }\end{array}$ & $\begin{array}{l}\text { 12,13-epoxy-9- } \\
\text { octadecenoic acid }\end{array}$ & $\begin{array}{l}\text { 12,13-epoxy-9,15- } \\
\text { octadecadienoic acid }\end{array}$ \\
\hline $\begin{array}{l}\omega \text {-oxo } \\
\text { fatty } \\
\text { acids }\end{array}$ & $\begin{array}{l}\text { 9-oxo nonanoic } \\
\text { acid }\end{array}$ & 9-oxo nonanoic acid & $\begin{array}{l}\text { (9Z)-12-oxo-9- } \\
\text { dodecenoic acid }\end{array}$ & $\begin{array}{l}\text { (9Z)-12-oxo-9- } \\
\text { dodecenoic acid }\end{array}$ \\
\hline $\begin{array}{l}\text { divinyl } \\
\text { ethers }\end{array}$ & colneleic acid (CA) & colnelenic acid (CnA) & - & - \\
\hline
\end{tabular}

In Table 4.3 the products from incubations with 10-, 14- and 17-hydroperoxides are shown.

Table 4.3: Products formed by conversions of 10-, 14-, and 17-hydroperoxides derived from $\mathrm{C}_{22}$ fatty acid DHA by BfEAS.

\begin{tabular}{llll}
\hline & \multicolumn{1}{c}{ 10-HPDHA } & \multicolumn{1}{c}{ 14-HPDHA } & \multicolumn{1}{c}{ 17-HPDHA } \\
\hline \multirow{3}{*}{ epoxy } & $(4 Z, 7 Z, 13 Z, 16 Z, 19 Z)-10-$ & $(4 Z, 7 Z, 10 Z, 16 Z, 19 Z)-14-$ & $(4 Z, 7 Z, 10 Z, 13 Z, 19 Z)-17-$ \\
alcohols & hydroxy-11,12-epoxy- & hydroxy-12,13-epoxy- & hydroxy-15,16-epoxy- \\
& $4,7,13,16,19-$ & $4,7,10,16,19-$ & $4,7,10,13,19-$ \\
& docosapentaenoic acid & docosapentaenoic acid & docosapentaenoic acid \\
\hline
\end{tabular}

Thus, one may conclude that BfEAS is an unspecific enzyme like PpAOS1. However, in order to confirm this hypothesis it is essential to determine the substrate preference of BfEAS by analyzing the kinetic parameters for 9-hydroperoxides derived from LA and $\alpha$-LeA as well as for 10- and 14hydroperoxides derived from DHA. Unfortunately measurements with 10- and 14-HPDHA as substrates could not be performed in frame of this thesis due to the limited amount of substrates available. Also, measurements of initial time-dependent consumptions of 9-hydroperoxides appeared to be difficult, because BfEAS has a broad product spectrum. As mentioned above beside 
EAS products (epoxy alcohols) also HPL products ( $\omega$-oxo fatty acids) as well as DES products (divinyl ether) were detected. Therefore it is very difficult to get reasonable data for $\mathrm{k}_{\text {cat }}$, because the broad absorption maxima ( $252 \mathrm{~nm}$ to $268 \mathrm{~nm}$ ) of the divinyl ethers that overlaps with those of the hydroperoxide substrates (234 nm).

\subsubsection{Mutagenesis}

In analogy to the mutagenesis analysis of PpAOS1, PpHPL, (StDES) and AtAOS essential residues in BfEAS were substituted to analyze if site-directed mutagenesis have an effect on the product formation.

Sequence alignments showed that specific amino acid residues important for product specificity in AtAOS as mentioned above (Lee et al., 2008), were also conserved in BfEAS (see Figure 3.17). To analyze the importance of these residues in BfEAS site-directed mutagenesis were performed. BfEAS contains the AOS-specific $F$, that is thought be essential for AOS activity by stabilizing intermediary formed substrate radicals (Lee et al., 2008). This residue was substituted by the HPL-specific L (BfEAS_F58L). In addition an in EAS conserved P located at position 75 in BfEAS was substituted by a HPL-specific A (BfEAS_P75A) and the AOS-specific S (BfEAS_P75S).

Lee and coworkers proposed that the conserved F, which is essential for AOSactivity, is located at position 60 in the BfEAS-sequence (AtAOS_F137) (Lee et al., 2008). This contradicts the alignments in this study where this particular F was located at position 58 in BfEAS. In order to investigate the molecular function of this amino acid in addition to that of the F58 a F60L variant was generated.

Toporkova and coworkers determined another strictly conserved F in tomato AOS (LeAOS_F295) (Toporkova et al., 2008). This F is also strictly conserved in AOS and in HPLs except in PpHPL where L is at this position. DES have a conserved I at this position. To analyze the importance of this $F$ BfEAS_F223I and BfEAS_F223L variants were generated.

At position 224 an N-residue is located in BfEAS. This $\mathrm{N}$ is highly conserved in all CYP74-enzymes and kinetic analyzes of AtAOS_N321Q showed that enzyme activity was abolished (Lee et al., 2008). Therefore the N224 in BfEAS was substituted by Q (BfEAS_N224Q).

Additionally to the site-directed mutagenesis of the activity determining amino acids residues a truncation variant was generated. CYP74-enzymes contain in contrast to classical P450-enzymes an insertion of 9AS in the near of the heme-binding loop. To analyze the molecular function of this insertion the BfEAS_9AS-deletion variant was generated and analyzed.

The generated BfEAS variants were purified and then analyzed by spectroscopic analysis and product analysis. 


\subsubsection{Spectroscopic anaylsis (UV/vis and CD)}

Spectroscopic analysis of BfEAS variants were performed to get information about the influence of site-directed mutagenesis on the proteinfolding and the cofactor binding.

UV/vis spectra of both variants (BfEAS_F58L and BfEAS_9AS-deletion) show absorption maxima for Soret-band and $\gamma$-band. In comparison to wild type the maxima for $\delta$-band in both variants are considerably low. Unlike the wild type BfEAS_F58L shows no absorption maxima for $\alpha$ - and $\beta$-bands and BfEAS_9AS-deletion only small absorption maxima for $\beta$-band (see Figure 3.44). This data might indicate that the amino acid exchanges resulted in an altered micro-environment of the heme cofactor. However, the enzyme variants apparently contain hemeiron in its low-spin state, because signals at $630 \mathrm{~nm}$, which were characteristic for high-spin iron-III-complexes were not detected.

The heme content of BfEAS_F58L is $16 \%$ and of BfEAS_9AS-deletion $2 \%$. Therefore the heme contents of the variants are lower than the content of wild type (37\%). The strong decrease of the heme content in BfEAS_9AS-deletion variant suggest that the deletion of the CYP74 9AS-insert, which is near the heme-binding loop, seem to have a significantly influence on proteinfolding.

Like the wild type the CD spectra of the variants (see Figure 3.45 ) showed characteristics for proteins with a mainly $\alpha$-helical fold (Panagakou et al., 2012).

\subsubsection{Analysis of products (radio-HPLC and RP-HPLC/MS²)}

The products formed by incubations of BfEAS variants with 9- and 13-hydroperoxides derived from LA and $\alpha$-LeA was analyzed by radio-HPLC and RP-HPLC/MS ${ }^{2}$.

The decrease of EAS activity in BfEAS_F58L variant by incubations with $\mathrm{C}_{18}$ fatty acid derived hydroperoxides indicates that the $\mathrm{F}$ at position 58 is essential for enzyme activity as it was also reported for AtAOS_F137L (Lee et al., 2008). The F is reported to stabilize the intermediately formed carbon-centered substrate radicals. Substitution of the aromatic F with HPL characteristic aliphatic L yielded a protein that cannot stabilize the substrate radical and therefore forms an unstable hemiacetal, which decomposes to short chain aldehydes (Grechkin and Hamberg, 2004). It was expected that mutagenesis of the EAS-specific $F$ to L (BfEAS_F58L) might lead to an increase of the inherent HPL- or DESactivity which was found as sideactivity in BfEAS wild type. However, radio-HPLC analysis of products formed from incubations with 9-and 13-hydroperoxides with this variant showed no significant increase of $\omega$-oxo fatty acids or divinyl ethers (see Figure 3.48, Figure 3.49 and Figure 9.4).Nevertheless substitution of $F$ at position 58 by $L$ does not lead to an inactive protein since a decrease of the respective hydroperoxide substrates was observed. Incubations of BfEAS_P75A variant with 9- and 13-hydroperoxides showed similar product patterns like BfEAS_F58L. For substitution of EAS-specific $P$ by HPL-specific $A$ it was expected that EASactivity decreases and HPLactivity increases. However, the analyses showed that inherent HPLactivity showed no 
significantly increases and that the product patterns were like that of BfEAS_F58L.The F58 and the P75 seem to be essential determinants for the formation of epoxy alcohols in BfEAS. Substitution of these determinants influenced the reaction mechanism in that way that EAS-route is disturbed and that the inherent activities (DES and HPL) appear to be unaffected.

Analysis of BfEAS_9AS-deletion variant showed surprisingly that this enzyme variant still exhibits catalytic activity (see Figure 3.48, Figure 3.49and Figure 9.4). The product pattern was similar to wild type, but the deletion-variant formed the products in lower amounts than the wild type does indicating that the activity was highly impaired. BfEAS_9AS-deletion showed with hydroperoxides derived from LA strong decreases of the epoxy alcohol, whereas with hydroperoxides derived from $\alpha$ LeA only slight decreases of epoxy alcohol were detected. Kinetic analysis of the deletion variant could verify the lower activity of BfEAS_9AS-deletion variant.

Radio-HPLC analyses of other generated variants (see Table 3.8) showed product patterns which were similar to that of wild type. Lee and coworkers reported for AtAOS that the substitution of $\mathrm{N}$ by Q (AtAOS_N321Q) abolish > $95 \%$ activity (Lee et al., 2008). Radio-HPLC and RP-HPLC/MS ${ }^{2}$ analyses indicated that the BfEAS_N224Q variant exhibits a similar catalytic activity as the wild type. Thus these results suggest that the postulated concept for N/Q-substitution may not be applicable to all CYP74-enzymes. To investigate this aspect in more detail, additionally steady state kinetic analysis of BfEAS_N224Q is essential. Lee showed that the respective F for enzyme activity alteration is in BfEAS at position 60(Lee et al., 2008). Incubations of BfEAS_F60L with 9- and 13-hydroperoxides showed similar product patterns like wild type. The results of the product analysis performed in this work suggest that the respective $\mathrm{F}$ is at position 58 (BfEAS_F58L) and not at position 60.

As shown in Table 3.8 the amount of products formed from hydroperoxides derived from LA was lower compared to that formed from LeA-analogous. This indicates that this enzyme variant might have a substrate preference for LeA-derived hydroperoxides. 


\section{Summary}

In this study recombinant CYP74-enzymes AOS1, AOS2 and HPL from the moss P. patens, DES from S. tuberosum and EAS from the lancelet B. floridae were expressed in E. coli and purified for further analysis. PpAOS1 and BfEAS were biochemically characterized. Substrate specificities against hydroperoxides derived from $C_{18}$ fatty acids LA and $\alpha$-LeA (PpAOS1 and BfEAS), $C_{20}$ fatty acid ARA (PpAOS1) as well as $\mathrm{C}_{22}$ fatty acid DHA (BfEAS) and pH optima were determined. Both enzymes showed slightly acidic pH (PpAOS1 6.5 and BfEAS 6.75). UV/vis and CD spectroscopy analysis were performed to characterize the heme content and structure of the enzymes.

Analysis of products of AOS, DES, EAS and HPL wild types showed that these enzymes have inherent side activities of the other enzymes beside their main enzyme activity. Thus PpHPL has inherent AOSactivity, PpAOS1 as well as StDES have inherent HPLactivity, whereas BfEAS has inherent HPLand DESactivity.

Site-directed mutagenesis was performed in order to identify amino acid residues essential for catalytic activity. Analysis of products of conversions of PpAOS1 with 9-and 13-hydroperoxides derived from LA and $\alpha$-LeA showed that enzyme activity can be altered from AOS to HPL by substitution of an essential determinant (PpAOS1_F93L). This mutagenesis concept was reported before for an AOS from Arabidopsis (AtAOS) with specificity for 13-hydroperoxides (Lee et al., 2008). In the present study this concept has been extended to AOS with specificity for 9-and 13hydroperoxide. The results showed that this concept works for both specific and unspecific enzymes. For PpHPL this concept was not applicable suggesting that for the alteration of HPL activity other determinants were essential.

Site-directed mutagenesis of StDES for product analysis generated mutants that had very low or no activity to perform the product analysis.

All purified variants of BfEAS were catalytically active. Product analysis showed that the mutational concept of AtAOS (Lee et al., 2008) can also be applied to BfEAS. It was observed that EASactivity decreased by mutating the conserved F (BfEAS_F58L) and the conserved P (BfEAS_P75A). But unlike in AtAOS or in PpAOS1 no significant increase of HPLactivity was detected, because in comparison to the other formed products the amount of oxo fatty acids was nearly the same. DESactivity like in the wild type was not detected in the variants. The results suggest like in PpAOS1 that the concept can be applied to unspecific enzymes. BfEAS is like PpAOS1 and unspecific enzyme.

The deletion of the 9AS-insert, which is characteristic for CYP74-enzyme, had no influence on enzymeactivity. The BfEAS_9AS-deletion variant was still active.

Product analysis by RP-HPLC/MS ${ }^{2}$ confirmed the formation of epoxy alcohols by conversions of BfEAS with 9- and 13-hydroperoxides derived from LA and $\alpha$-LeA. The detected fragment patterns were similar to that of wild type (Cristea and Oliw, 2006, Oliw et al., 2006). 
Product analysis showed that the catalytic trajectories of PpAOS1 and PpHPL as well as BfEAS, DES and HPL are closely interconnected and can be interconverted by exchanges of special single amino acids. 


\section{Outlook}

In order to get further insights into the CYP74-catalysis different biochemical and biophysical experiments might be performed: On the one hand different heme intermediates formed during catalysis might be analyzed by performing time resolved UV/vis spectroscopy employing the stopped flow technique. Data derived from those experiments might further analyzed by performing freezequench EPR-spectroscopy. In similar experiments on the other hand different substrate intermediates might be identified. Here it will be of special interest to investigate whether the different catalytic trajectories occur by a pure radical pathway or if also cationic intermediates might be formed as proposed recently (Brash, 2009).

Additionally, X-ray crystallography might be used to determine the 3D-structure of other CYP74, because only AOS-structures have been solved in the field of the CYP74-enzymes (AtAOS and PaAOS) (Lee et al., 2008, Li et al., 2008).

To get a further inside into the physiological role of the fourth CYP74-enzyme EAS was transferred into A. thaliana. As it is known that EAS derived oxylipins are thought to be involved in pathogen defense reactions (Feussner and Wasternack, 2002, Blée, 1998b). Thus it will be of interest to investigate if the recombinant plants are more resistant compared to wild type plants. 


\section{References}

Andreou, A., Brodhun, F. \& Feussner, I. (2009). Biosynthesis of oxylipins in non-mammals. Prog Lipid Res, 48: 148-170.

Anterola, A., Göbel, C., Hornung, E., Sellhorn, G., Feussner, I. \& Grimes, H. (2009). Physcomitrella patens has lipoxygenases for both eicosanoid and octadecanoid pathways. Phytochemistry, 70: 40-52.

Ausubel, F.M., Brent, R.E., Kingston, D.D., Seidmann, J.R., Smith, J.A. \& Struhl, K. (1993). Current Protocolls in Molecular Biology, New York: Green Publishing Associates and John Wiley and Sons Inc.

Bandara, P.K., Takahashi, K., Sato, M., Matsuura, H. \& Nabeta, K. (2009). Cloning and functional analysis of an allene oxide synthase in Physcomitrella patens. Biosci Biotechnol Biochem, 73: 2356-9.

Birnboim, H.C. \& Doly, J. (1979). A rapid alkaline extraction procedure for screening recombinant plasmid DNA. Nucleic Acids Res, 7: 1513-23.

Blée, E. (1998a). Biosynthesis of phytooxylipins: the Peroxygenase pathway. Fett/Lipid, 100: 121-127.

Blée, E. (1998b). Phytooxylipins and plant defense reactions. Prog Lipid Res, 37: 33-72.

Blée, E. \& Joyard, J. (1996). Envelope membranes from spinach chloroplasts are a site of metabolism of fatty acid hydroperoxides. Plant Physiol, 110: 445-454.

Bradford, M.M. (1976). A rapid and sensitive method for the quantitation of microgram quantities of proteins utilizing the principle of protein-dye binding. Anal Biochem, 72: 248-254.

Brash, A.R. (2009). Mechanistic aspects of CYP74 allene oxide synthases and related cytochrome P450 enzymes. Phytochemistry, 70: 1522-1531.

Brash, A.R., Baertschi, S.W., Ingram, C.D. \& Harris, T.M. (1988). Isolation and characterization of natural allene oxides: unstable intermediates in the metabolism of lipid hydroperoxides. Proc Natl Acad Sci USA, 85: 3382-3386.

Brodhun, F., Gobel, C., Hornung, E. \& Feussner, I. (2009). Identification of PpoA from Aspergillus nidulans as a fusion protein of a fatty acid heme dioxygenase/peroxidase and a cytochrome P450. J Biol Chem, 284: 11792-11805.

Bullock, W.D., Fernandez, J.M. \& Hort, J.M.S. (1987). XL1-Blue: A high efficiency plasmid transformants recA Escherichia coli strain $\beta$-galactosidase selection. BioTechniques, 5: 376378.

Chechetkin, I.R., Blufard, A., Hamberg, M. \& Grechkin, A.N. (2008). A lipoxygenase-divinyl ether synthase pathway in flax (Linum usitatissimum L.) leaves. Phytochemistry, 69: 2008-2015.

Cho, K.-B., Lai, W., Hamberg, M., Raman, C.S. \& Shaik, S. (2011). The reaction mechanism of allene oxide synthase: Interplay of theoretical $\mathrm{QM} / \mathrm{MM}$ calculations and experimental investigations. Arch Biochem Biophys, 507: 14-25. 
Cristea, M. \& Oliw, E.H. (2006). A G316A mutation of manganese lipoxygenase augments hydroperoxide isomerase activity: Mechanism of biosynthesis of epoxyalcohols. $J$ Biol Chem, 281: 17612-17623.

Dembitsky, V.M. (1993). Lipids of bryophytes. Prog Lipid Res, 32: 281-356.

Denisov, I.G., Makris, T.M., Sligar, S.G. \& Schlichting, I. (2005). Structure and chemistry of cytochrome P450. Chem Rev, 105: 2253-2278.

Fammartino, A., Cardinale, F., Göbel, C., Mene-Saffrane, L., Fournier, J., Feussner, I. \& EsquerreTugaye, M.-T. (2007). Characterization of a divinyl ether biosynthetic pathway specifically associated with pathogenesis in tobacco. Plant Physiol, 143: 378-388.

Feussner, I. \& Wasternack, C. (1998). Lipoxygenase catalyzed oxygenation of lipids. Fett/Lipid, 100: 146-152.

Feussner, I. \& Wasternack, C. (2002). The lipoxygenase pathway. Annu Rev Plant Biol, 53: 275-297.

Froehlich, J.E., Itoh, A. \& Howe, G.A. (2001). Tomato allene oxide synthase and fatty acid hydroperoxide lyase, two cytochrome P450s involved in oxylipin metabolism, are targeted to different membranes of chloroplast envelope. Plant Physiol, 125: 306-317.

Funk, C.D. (2001). Prostaglandins and leukotrienes: advances in eicosanoid biology. Science, 294: 1871-5.

Gerwick, W.H. (1994). Structure and biosynthesis of marine algal oxylipins. Biochim Biophys Acta, 1211: 243-55.

Girke, T., Schmidt, H., Zähringer, U., Reski, R. \& Heinz, E. (1998). Identification of a novel $\Delta 6$-acylgroup desaturase by targeted gene disruption in Physcomitrella patens. Plant J, 15: 39-48.

Göbel, C., Feussner, I., Hamberg, M. \& Rosahl, S. (2002). Oxylipin profiling in pathogen-infected potato leaves. Biochim Biophys Acta, 1584: 55-64.

Göbel, C., Feussner, I., Schmidt, A., Scheel, D., Sanchez-Serrano, J., Hamberg, M. \& Rosahl, S. (2001). Oxylipin profiling reveals the preferential stimulation of the 9-lipoxygenase pathway in elicitor-treated potato cells. J Biol Chem, 276: 6267-6273.

Gogolev, Y.V., Gorina, S.S., Gogoleva, N.E., Toporkova, Y.Y., Chechetkin, I.R. \& Grechkin, A.N. (2011). Green leaf divinyl ether synthase: gene detection, molecular cloning and identification of a unique CYP74B subfamily member. Biochim Biophys Acta.

Gottesman, S., Halpern, E. \& Trisler, P. (1981). Role of sulA and sulB in filamentation by lon mutants of Escherichia coli K-12. J Bacteriol, 148: 265-73.

Grechkin, A.N. (2002). Hydroperoxide lyase and divinyl ether synthase. Prostaglandins Other Lipid Mediat, 68-69: 457-570.

Grechkin, A.N. \& Hamberg, M. (2004). The "heterolytic hydroperoxide lyase" is an isomerase producing a short-lived fatty acid hemiacetal. Biochim Biophys Acta, 1636: 47-58. 
Grechkin, A.N., Kuramshin, R.A., Safonova, E.Y., Latypov, S.K. \& Ilyasov, A.V. (1991). Formation of ketols from linolenic acid 13-hydroperoxide via allene oxide. Evidence for two distinct mechanisms of allene oxide hydrolysis. Biochim Biophys Acta, 1086: 317-325.

Grechkin, A.N., Mukhtarova, L.S., Latypova, L.R., Gogolev, Y., Toporkova, Y.Y. \& Hamberg, M. (2008). Tomato CYP74C3 is a Multifunctional Enzyme not only Synthesizing Allene Oxide but also Catalyzing its Hydrolysis and Cyclization. ChemBioChem, 9: 2498-2505.

Hamberg, M. (1987). Mechanism of corn hydroperoxide isomerase: detection of 12,13(S)-oxido9(Z),11-octadecadienoic acid. Biochim Biophys Acta, 920: 76-84.

Hamberg, M. (1997). Myoglobin-catalyzed bis-allylic hydroxylation and epoxidation of linoleic acid. Arch Biochem Biophys, 344: 194-199.

Hamberg, M. (1998). A pathway for biosynthesis of divinyl ether fatty acids in green leaves. Lipids, 33: 1061-1071.

Hamberg, M. (1999). An epoxy alcohol synthase pathway in higher plants: Biosynthesis of antifungal trihydroxy oxylipins in leaves of potato. Lipids, 34: 1131-1142.

Hamberg, M. (2004). Isolation and structures of two divinyl ether fatty acids from Clematis vitalba. Lipids, 39: 565-9.

Hamberg, M. \& Fahlstadius, P. (1990). Allene oxide cyclase: a new enzyme in plant lipid metabolism. Arch Biochem Biophys, 276: 518-526.

Hamberg, M. \& Gardner, H.W. (1992). Oxylipin pathway to jasmonates: biochemistry and biological significance. Biochim Biophys Acta, 1165: 1-18.

Hamberg, M., Miersch, O. \& Sembdner, G. (1988). Absolute Configuration of 12-Oxo-10,15 (Z)phytodienoic Acid. lipids, 23: 521-524.

Hanano, A., Burcklen, M., Flenet, M., Ivancich, A., Louwagie, M., Garin, J. \& Blee, E. (2006). Plant seed peroxygenase is an original heme-oxygenase with an EF-hand calcium binding motif. I Biol Chem, 281: 33140-33151.

Hong, S., Lu, Y., Yang, R., Gotlinger, K.H., Petasis, N.A. \& Serhan, C.N. (2007). Resolvin D1, protectin $D 1$, and related docosahexaenoic acid-derived products: Analysis via electrospray/low energy tandem mass spectrometry based on spectra and fragmentation mechanisms. J Am Soc Mass Spectrom, 18: 128-44.

Howe, G.A. \& Jander, G. (2008). Plant immunity to insect herbivores. Annu Rev Plant Biol, 59: 41-66.

Howe, G.A. \& Schilmiller, A.L. (2002). Oxylipin metabolism in response to stress. Curr Opin Plant Biol, 5: 230-6.

Hughes, R.K., Belfield, E.J., Ashton, R., Fairhurst, S.A., Göbel, C., Stumpe, M., Feussner, I. \& Casey, R. (2006a). Allene oxide synthase from Arabidopsis thaliana (CYP74A1) exhibits dual specificity that is regulated by monomer-micelle association. FEBS Lett, 580: 4188-4194.

Hughes, R.K., Belfield, E.J. \& Casey, R. (2006b). CYP74C3 and CYP74A1, plant cytochrome P450 enzymes whose activity is regulated by detergent micelle association, and proposed new rules for the classification of CYP74 enzymes. Biochem Soc Trans, 34: 1223-7. 
Hughes, R.K., De Domenico, S. \& Santino, A. (2009). Plant cytochrome CYP74 family: biochemical features, endocellular localisation, activation mechanism in plant defence and improvements for industrial applications. ChemBioChem, 10: 1122-33.

Iny, D., Grossman, S. \& Pinsky, A. (1993). Lipoxygenase of the thermophilic bacteria thermoactinomyces-vulgaris - properties and study on the active site. Int J Biochem, 25: 1325-1330.

Itoh, A. \& Howe, G.A. (2001). Molecular cloning of a divinyl ether synthase: Identification as a CYP74 cytochrome P450. J Biol Chem, 276: 3620-3627.

Itoh, A., Schilmiller, A.L., Mccaig, B.C. \& Howe, G.A. (2002). Identification of a jasmonate-regulated allene oxide synthase that metabolizes 9-hydroperoxides of linoleic and linolenic acids. J Biol Chem, 277: 46051-46058.

Kuroda, H., Oshima, T., Kaneda, H. \& Takashio, M. (2005). Identification and Functional Analyses of Two cDNAs That Encode Fatty Acid 9-/13-Hydroperoxide Lyase (CYP74C) in Rice. Biosci Biotechnol Biochem, 69: 1545-54.

Laemmli, U.K. (1970). Cleavage of structural proteins during the assembly of the head of bacteriophage T4. Nature, 227: 680-684.

Lang, I., Göbel, C., Porzel, A., Heilmann, I. \& Feussner, I. (2008). A lipoxygenase with linoleate diol synthase activity from Nostoc sp. PCC 7120. Biochem J, 410: 347-57.

Lee, D.-S., Nioche, P., Hamberg, M. \& Raman, C.S. (2008). Structural insights into the evolutionary paths of oxylipin biosynthetic enzymes. Nature, 455: 363-368.

Li, L., Chang, Z., Pan, Z., Fu, Z.-Q. \& Wang, X. (2008). Modes of heme binding and substrate access for cytochrome P450 CYP74A revealed by crystal structures of allene oxide synthase. Proc NatI Acad Sci USA, 105: 13883-13888.

Liavonchanka, A. \& Feussner, I. (2006). Lipoxygenases: Occurrence, functions and catalysis. J Plant Physiol, 163: 348-357.

Lottspeich, F., Engels, J.W. (2006). Bioanalytik: Spektrum Akademischer Verlag Heidelberg.

Marnett, L.J. (2008). Biochemistry: Divergence from the superfamily. Nature, 455: 300-1.

Matsui, K. (2006). Green leaf volatiles: hydroperoxide lyase pathway of oxylipin metabolism. Curr Opin Plant Biol, 9: 274-280.

Matsui, K., Kaji, Y., Kajiwara, T. \& Hatanaka, A. (1996). Developmental changes of lipoxygenase and fatty acid hydroperoxide lyase activities in cultured cells of Marchantia polymorpha. Phytochemistry, 41: 177-182.

Matsui, K., Minami, A., Hornung, E., Shibata, H., Kishimoto, K., Ahnert, V., Kindl, H., Kajiwara, T. \& Feussner, I. (2006). Biosynthesis of fatty acid derived aldehydes is induced upon mechanical wounding and its products show fungicidal activities in cucumber. Phytochemistry, 67: 649657.

Mosblech, A., Feussner, I. \& Heilmann, I. (2009). Oxylipins: Structurally diverse metabolites from fatty acid oxidation. Plant Physiol Biochem, 47: 511-517. 
Mullis, K., Faloona, F., Scharf, S., Saiki, R., Horn, G. \& Erlich, H. (1986). Specific enzymatic amplification of DNA in vitro: the polymerase chain reaction. Cold Spring Harb Symp Quant Biol, 51 Pt 1: 263-73.

Noordermeer, M.A., Van Dijken, A.J., Smeekens, S.C., Veldink, G.A. \& Vliegenthart, J.F. (2000). Characterization of three cloned and expressed 13-hydroperoxide lyase isoenzymes from alfalfa with unusual $\mathrm{N}$-terminal sequences and different enzyme kinetics. Eur J Biochem, 267: 2473-82.

Noordermeer, M.A., Veldink, G.A. \& Vliegenthart, J.F. (2001a). Fatty acid hydroperoxide lyase: A plant cytochrome P450 enzyme involved in wound healing and pest resistance. ChemBioChem, 2: 494-504.

Noordermeer, M.A., Veldink, G.A. \& Vliegenthart, J.F. (2001b). Spectroscopic studies on the active site of hydroperoxide lyase; the influence of detergents on its conformation. FEBS Lett, 489: 229-32.

Noverr, M.C., Erb-Downward, J.R. \& Huffnagle, G.B. (2003). Production of eicosanoids and other oxylipins by pathogenic eukaryotic microbes. Clin Microbiol Rev, 16: 517-33.

Ogorodnikova, A.V., Latypova, L.R., Mukhitova, F.K., Mukhtarova, L.S. \& Grechkin, A.N. (2008). Detection of divinyl ether synthase in Lily-of-the-Valley (Convallaria majalis) roots. Phytochemistry, 69: 2793-8.

Ohta, H., Shida, K., Peng, Y.-L., Furusawa, I., Shishiyama, J., Aibara, S. \& Morita, Y. (1990). The occurence of lipid hydroperoxide-decomposing activities in rice and the relationship of such activities to the formation of antifungal substances. Plant Cell Physiol, 31: 1117-1122.

Oliw, E.H., Garscha, U., Nilsson, T. \& Cristea, M. (2006). Payne rearrangement during analysis of epoxyalcohols of linoleic and alpha-linolenic acids by normal phase liquid chromatography with tandem mass spectrometry. Anal Biochem, 354: 111-126.

Panagakou, I., Touloupakis, E. \& Ghanotakis, D. (2012). Structural Characterization of Hydroperoxide Lyase in Dodecyl Maltoside by Using Circular Dichroism. The Protein Journal: 1-6.

Porta, H. \& Rocha-Sosa, M. (2001). Lipoxygenase in bacteria: a horizontal transfer event? Microbiol, 147: 3199-3200.

Richardson, T.H., Hsu, M.H., Kronbach, T., Barnes, H.J., Chan, G., Waterman, M.R., Kemper, B. \& Johnson, E.F. (1993). Purification and characterization of recombinant-expressed cytochrome P450 2C3 from Escherichia coli: 2C3 encodes the $6 \beta$-hydroxylase deficient form of P450 3b. Arch Biochem Biophys, 300: 510-516.

Sanger, F., Nicklen, S. \& Coulson, A.R. (1977). DNA Sequencing with chain-terminating inhibitors. Proc Natl Acad Sci USA, 74: 5463-5467.

Schaller, A. \& Stintzi, A. (2009). Enzymes in jasmonate biosynthesis - Structure, function, regulation. Phytochemistry, 70: 1532-1538.

Scholz, J. (2009). Diplomarbeit: Eigenschaften von CYP74-Enzymen in Arabidopsis thaliana und Physcomitrella patens. Abteilung Biochemie der Pflanze. Göttingen, Deutschland: GeorgAugust-Universität. 
Scholz, J., Brodhun, F., Hornung, E., Herrfurth, C., Stumpe, M., Beike, A., Faltin, B., Frank, W., Reski, R. \& Feussner, I. (2012). Biosynthesis of allene oxides in Physcomitrella patens. BMC Plant Biology, 12: 228.

Schückel, J., Rylott, E.L., Grogan, G. \& Bruce, N.C. (2012). A Gene-Fusion Approach to Enabling Plant Cytochromes P450 for Biocatalysis. ChemBioChem: n/a-n/a.

Senger, T., Wichard, T., Kunze, S., Göbel, C., Lerchl, J., Pohnert, G. \& Feussner, I. (2005). A multifunctional lipoxygenase with fatty acid hydroperoxide cleaving activity from the moss Physcomitrella patens. J Biol Chem, 280: 7588-7596.

Shibata, Y., Matsui, K., Kajiwara, T. \& Hatanaka, A. (1995). Purification and properties of fatty acid hydroperoxide lyase from green bell pepper fruits. Plant Cell Physiol, 36: 147-156.

Song, W.-C. \& Brash, A.R. (1991). Purification of an allene oxide synthase and identification of the enzyme as a cytochrome P-450. Sience, 253: 781-784.

Stumpe, M., Bode, J., Göbel, C., Wichard, T., Schaaf, A., Frank, W., Frank, M., Reski, R., Pohnert, G. \& Feussner, I. (2006a). Biosynthesis of C9-aldehydes in the moss Physcomitrella patens. Biochim Biophys Acta, 1761: 301-312.

Stumpe, M., Carsjens, J.-G., Göbel, C. \& Feussner, I. (2008). Divinyl ether synthesis in garlic bulbs. J Exp Bot, 59: 907-915.

Stumpe, M., Carsjens, J.-G., Stenzel, I., Göbel, C., Lang, I., Pawlowski, K., Hause, B. \& Feussner, I. (2005). Lipid metabolism in arbuscular mycorrhizal roots of Medicago truncatula. Phytochemistry, 66: 781-791.

Stumpe, M. \& Feussner, I. (2006). Formation of oxylipins by CYP74 enzymes. Phytochem Rev, 5: 347357.

Stumpe, M., Göbel, C., Demchenko, K., Hoffmann, M., Klösgen, R.B., Pawlowski, K. \& Feussner, I. (2006b). Identification of an allene oxide synthase (CYP74C) that leads to formation of $\alpha$ ketols from 9-hydroperoxides of linoleic and linolenic acid in below-ground organs of potato. Plant J, 47: 883-896.

Stumpe, M., Göbel, C., Faltin, B., Beike, A.K., Hause, B., Himmelsbach, K., Bode, J., Kramell, R., Wasternack, C., Frank, W., Reski, R. \& Feussner, I. (2010). The moss Physcomitrella patens contains cyclopentenones but no jasmonates: mutations in allene oxide cyclase lead to reduced fertility and altered sporophyte morphology. New Phytol, 188: 740-749.

Stumpe, M., Kandzia, R., Göbel, C., Rosahl, S. \& Feussner, I. (2001). A pathogen-inducible divinyl ether synthase (CYP74D) from elicitor-treated potato suspension cells. FEBS Lett, 507: 371-376.

Taiz, L., Zeiger, E. (2007). Plant Physiology Das Original mit Übersetzungshilfen: Spektrum Akademischer Verlag.

Toporkova, Y.Y., Gogolev, Y.V., Mukhtarova, L.S. \& Grechkin, A.N. (2008). Determinants governing the CYP74 catalysis: Conversion of allene oxide synthase into hydroperoxide lyase by sitedirected mutagenesis. FEBS Lett, 582: 3423-3428. 
Toporkova, Y.Y., Osipova, E.V., Mukhtarova, L., Gogolev, Y.V. \& Grechkin, A.N. (2010). Alteration of catalysis of CYP74C subfamily enzymes as a result of site-directed mutagenesis. Dokl Biochem Biophys, 435: 287-290.

Tsitsigiannis, D.I. \& Keller, N.P. (2007). Oxylipins as developmental and host-fungal communication signals. Trends Microbiol, 15: 109-118.

Vick, B.A. \& Zimmerman, D.C. (1976). Lipoxygenase and hydroperoxide lyase in germinating watermelon seedlings. Plant Physiol, 57: 780-788.

Vick, B.A. \& Zimmerman, D.C. (1984). Biosynthesis of jasmonic acid by several plant species. Plant Physiol, 75: 458-461.

Wasternack, C. (2007). Jasmonates: An update on biosynthesis, signal transduction and action in plant stress response, growth and development. Ann Bot, 100: 681-697.

Wasternack, C. \& Kombrink, E. (2010). Jasmonates: Structural requirements for lipid-derived signals active in plant stress responses and development. ACS Chem Biol, 5: 63-77.

Weber, H., Chetelat, A., Caldelari, D. \& Farmer, E.E. (1999). Divinyl ether fatty acid synthesis in late blight-diseased potato leaves. Plant Cell, 11: 485-493.

Wennman, A. \& Oliw, E.H. (2012). Secretion of two novel enzymes, manganese 9S-Lipoxygenase and epoxy alcohol synthase, by the rice pathogen Magnaporthe salvinii. J Lipid Res.

Werck-Reichhart, D. \& Feyereisen, R. (2000). Cytochromes P450: a success story. Genome Biology, 1: reviews3003.1-reviews3003.9.

Wichard, T., Göbel, C., Feussner, I. \& Pohnert, G. (2005). Unprecedented lipoxygenase/hydroperoxide lyase pathways in the moss Physcomitrella patens. Angew Chem Int Ed Engl, 44: 158-161.

Ziegler, J., Wasternack, C. \& Hamberg, M. (1999). On the specificity of allene oxide cyclase. Lipids, 34: 1005-1015. 


\section{Acknowledgements}

I like to thank all who supported and encouraged me during the completion of this work.

I am grateful to Prof. Dr. Ivo Feußner who gives me the opportunity to work on this interesting project. I also want to thank for his supervision and guidance, helpful discussions and his interest in the progress of the project.

I thank Prof. Dr. Kai Tittmann for being the second referee of my thesis as well as for being member of my thesis committee.

I would like to thank Prof. Dr. Marina Bennati for being member of my thesis committee.

The international research training group (IRTG 1422) "Metal Sites in Biomolecules: Structures, Regulation and Mechanisms" I want to thank for funding and also for organizing all the interesting and helpful seminars, workshops and symposia.

The Göttingen Graduate School of Neurosciences, Biophysics and Molecular Biosciences (GGNB) I thank for the GGNB Bridging Fund and for the opportunity to take part in the interesting courses and events in the framework of the graduate school.

I thank Dr. Florian Brodhun for his great help, advice, patience, his encouragement and for answering my questions.

Dr. Ellen Hornung I am thankful for her helpful tips and tricks in the lab and for all the delicious cakes and cookies, especially for the "extra-free-versions".

I also want to thank Dr. Danilo Meyer for helping and explaining me CD spectroscopy.

I thank my lab mate Steffen Kawelke for bringing the "magic power" in our lab.

Sabine Freitag I am thankful for her help in the "fight" with the HPLCs.

I thankGerd and Christoph Mader for their help with computer problems and for keep the machines running. 
I thank all members of the Department for the nice atmosphere in the lab.

Thanks Hamze Beati and Stefan Schneider for the coffee breaks, even if it wereat the endalwaysless.

Furthermore I like to thank Prof. Dr. Feußner, Dr. Florian Brodhun and Rainer Scholz for prove reading of my thesis and valuable comments.

At the end I want to thank my family for their support and understanding and that they always trust in me.

Thanks to everyone that I may forgot. 


\section{Appendix}

\subsection{Sequences for alignment}

\subsubsection{AtAOS}

MASISTPFPISLHPKTVRSKPLKFRVLTRPIKASGSETPDLTVATRTGSKDLPIRNI PGNYGLPIVGPIKDRWDY FYDQGAEEFFKSRIRKYNSTVYRVNMPPGAFIAENPQVVALLDGKSFPVLFDVDKVEKKDLFTGTYMPSTELTGG YRILSYLDPSEPKHEKLKNLLFFLLKS SRNRIFPEFQATYSELFDSLEKELSLKGKADFGGSSDGTAFNFLARAF YGTNPADTKLKADAPGLITKWVLFNLHPLLS I GLPRVIEEPLI HTFS LPPALVKS DYQRLYEF FLESAGE ILVEA DKLGISREEATHNLLFATCFNTWGGMKI LFPNMVKRIGRAGHQVHNRLAEEIRSVIKSNGGELTMGAIEKMELTK SVVYECLRFEPPVTAQYGRAKKDLVIESHDAAFKVKAGEMLYGYQPLATRDPKIFDRADEFVPERFVGEEGEKLL RHVLWSNGPETETPTVGNKQCAGKDFVVLVARLFVIEIFRRYDSFDIEVGTSPLGSSVNFSSLRKASF

\subsubsection{PaAOS}

MDPSSKPLREIPGSYGIPFFQPIKDRLEYFYGTGGRDEYFRSRMQKYQSTVFRANMP PGPFVSSNPKVIVLLDAK SFPILFDVSKVEKKDLFTGTYMPSTKLTGAYRVLSYLDPSEPRHAQLKNLLFEMLKNSSNRVI PQFETTYTELFE GLEAELAKNGKAAFNDVGEQAAFRFLGRAYFNSNPEETKLGTSAPTLISSWVLFNLAPTLDLGLPWFLQEPLLHT FRLPAFLIKSTYNKLYDYFQSVATPVMEQAEKLGVPKDEAVHNILFAVCFNTFGGVKILFPNTLKWIGVAGENLH TQLAEEIRGAIKSYGDGNVTLEAIEQMPLTKSVVYESLRIEP PVP PQYGKAKSNFT IESHDATFEVKKGEMLFGY QPFATKDPKVFDRPEEFVPDRFVGDGEALLKYVWWSNGPETESPTVENKQCAGKDFVVLITRLFVIELFRRYDSF EIELGESPLGAAVTLTFLKRASI

\subsubsection{LeAOS3}

MANTKDSYHIITMDTKESS I PSLPMKEI PGDYGVPFFGAIKDRYDFHYNQGADEFFRSRMKKYDSTVFRTNVPPG PENARNSKVVVLVDAVSYPILFDNSQVDKENYFEGTFMS SPSFNGGYKVCGFLGTSDPKHTTLKGLFLSTLTRLH DKFIPIFTTS ITSMFTSLEKELSEKGTSYFNP I GDNLSFEFLFRLFCEGKNP I DTSVGPNGPKIVDKWVFLQLAP LISLGLKFVPNFLEDLVLHTFPLPY I LVKRDHQKLYNAFYNSMKD I LDEAEKLGVKRDEACHNFVFLAGFNSYGG LKVFFPSLIKWIGTSGPSLHARLVKEIRTAVKEAGGVTLSAIDKMPLVKSVVYETLRMDPPVPFQTVKARKNI I I TNHESSFLIKKDELIFGYQPLATKDSKVFKNAEEFNPDRFVGGGEKLLKYVYWSNGKEI DNPSVNDKQCPGKDLI VLMGRLLVVEFFMRYDTFEVEFGKLLLGSKVTEKSLTKATS

\subsubsection{PpAOS1}

MAVPSSKLPLKAI PGDYGVPYFGAIKDRLDYFWLQGEEQFYRSRMAKYNSTVFRVNMP PGPPISEHPQVICLLDQ KSFPILFDVSKVEKKDVFTGTYMPSVSFTSGYRVCSYLDPSEERHTKLKQWCEEVIAMNGRNFLPEFHKS IEESM VLWETSLAKGEKTSVSDEVKQFAFNFLMRAVCHHDPAAPGEYSLGRNGGPYATAWANPQLAPIAGQTGLPHVVEE LVLHTVPLPSALVKKNYDALYNFIKNYATEALDRAEAMGIERNDATANLLFFLCFNAYGGFSIFFPLITILISSC GPELMHDLHDEVTKAVAATDGKVTLQS IENMPLVKSVVYEAFRFKPPVPYQYGKAKFDFTIENHENSFEVKKGEM LYGYQPIVMHDPKVFSDPDQFLPRRFMGPDGEKLIKYIFWSNGYETDEPTTANKQCAGKDLVVTMARAFVAEMFL RYKEYTLTMEGAGNATKVFFSDLKK 


\subsubsection{PpAOS2}

MAVPVSNLPLRAIPGGYGISYLGAIKDRLDYFWIQGEEEFYRSRVEKYNSTVERVSMPPGPPIAKDARVICVLDQ KSFP I LFDVNKCEKRDLFLGTYMPDLSYTSGHRVLSYLDPSEVRHEKLKQWCFDLIARNGRKF LPEFHTAMEESF AVWEEAMEKGENANLSEEVQQFAFNFLVRAVLHHDPVAPGEAS LGKNGGPYASAWHGPQLAP IAGQTGLPHAVEE LLHTIRLPSSVVKEQYDALYNFFKTYGGEELDRAVALGI KRDDAIANLLFLLGFNAYGGFNFFFPQLTGHIAQCG PELMHELHEEVVAAVQATEGKVTPKS LENMPLLSSVVYEGFRMKP PVPYQYARAKTDFLIESHENSEEVKKGEML YGFQPYVMHDPNVFENPDKFLPRRFMGPEGEALLGNVFWSNGRETDDPTVHDKQCAGKDLAVTISRAYVAEMFLR YKEFTLEVQGSGVQTTLLFSALQKA

\subsubsection{AtHPL}

MDLVDKRDVLIGDFRPSLGFYGGVRVGVYLDTTEPKHAKIKGFAMETLKRSSKVWLQELRSNLNIFWGTIESEIS KNGAASYIFPLQRCIFSFLCASLAGVDASVSPDIAENGWKT INTWLALQVI PTAKLGVVPQPLEEILLHTWPYPS LLIAGNYKKLYNF I DENAGDCLRLGQEEFGLTRDEAIQNLLFVLGFNAYGGFSVFLPSLIGRITGDNSGLQERIR TEVRRVCGSGSDLNFKTVNEMELVKSVVYETLRF S P PVPLQFARARKDFQI S S HDAVFEVKKGELLCGYQPLVMR DANVFDEPEEFKPDRYVGETGSELLNYLYWSNGPQTGTPSASNKQCAAKDIVTLTASLLVADLFLRYDTITGDSG SIKAVVKAK

\subsubsection{LeHPL}

MNSAPLSTPAPVTLPVRSI PGSYGLPLVGPIADRLDYFWFQKPENFFTKRMEKHKSTVFRTNVPPCFPFFGSVNP NVVAVLDVKSFSHLFDMEIVEKANVLVGDFMPSVVYTGDMRVCAYLDTSEPKHAQIKNFSQDI LKRGSKTWVPTL LKELDTMFTTFEADLSKSNTASLLPALQKFLFNFFSLTILGADPSVSPEIANSGYIFLDSWLAIQLAPTVS IGVI QPLEEILVHSFAYPFFLVKGNYEKLVQFVKNEAKEVLSRAQTGFQLTEQEA I HNLLFILGFNAFGGFS IFLPTLL GNLGDEKNADMQEKLRKEVRDKVGVNPENLSFESVKEMELVQSFVYETLRLSP PVP SQYARARKDFKLSSHDSVY EIKKGELLCGYQPLVMKDPKVFDEPEKFVLERFTKEKGKELLNYLFWSNGPQTGRPTESNKQCAAKDMVTLTASL IVAYIFQKYDSVSFSSGSLTSVKKAS

\subsubsection{PpHPL}

MDRTLVLTCTTTCSHSAFRQSALPSNTS ISVRLGTCSVRTQKRRTVVASLGNIETTSTSTVGQESNLPLREIPGS YGI PYLSQLLDRWTFFYREGEPQFWQSRMAKYGSTVIRSNMPPGWFWTDSRC IMLLDQKSYPTVFDYDKVDKYKA FAGT IMPSTEYNGGYEVCAYLDASDKKHEQLKGYCEELLKFSSSKWAREFHTAISETFNQWEGKLAQKTPALINP TLPESLFSFVINALTTARFDDSS IPDAEKPVCGDLQKWAGFQLMPVIRTGAPIYIEEMLHVAPIPASLTKGGYDK MVVFLQKYAAETLSIAEKFGLSQDEAVHNLIFFLILNAHGGFCRFLPVILREVAKNGQLQADLREEVRAAVKASG SDQVTMKAVMNDMPLVASTVFEALRFDPPVPFQYARAKKDFIIESHDARYQIKTGDFLGGVNYMVSRDPKVFTDR PNEFNARRFMGPEGDKLLAHLVWSNGRQTDETTVYTKQCAGKEIVPLTGRLLLAELFMRFDSFNIEGLEMEATFT SLTPRSD

\subsubsection{StDES}

MSSYSELSNLPIREIPGDYGFPI ISAIKDRYDYFYNQGEDAWFHNKAEKYKSTVVKINMAPGPFTSNDYKLVAFL DANSFVCMFDNSLIDKTDTLGGTFKPGKEYYSGYRPVAFIDTKDPNHAALKGYILSAFAKRHNLFIPLFRNSLSD HLFNNLEKQVTEQGKS DFNALLPTMTFNFIFRLLCDQTNPS DTVLGAQGPEHLRKWLFPQLI PSLSAKKLPNI IE DTLFHNFLI PFGFIKS DYNKLVDAFSKSAVS I LDEAEKLGIKREEAVQN I LFLVG INMFAGLNAFS PHLFRFVGE AGASLHTQLAKEIRTVIKEEGGAITLSAINKMS LVKSVVYETLRLRP PVPLQYGKAKKDFMVQSHDASYKINKGQ FVVGYQPMASRDPKIFANPDEFVPDRFMNDGEKMLKHVLWSNGRETENPAPDNKQCPGKDLVHLLGRLI LVEFFM RYDTFTVEITPLFRAPNVAFKTLTKASK 


\subsubsection{AsDES}

MSTSNGSTENIQKPLRKIPDITGTPILTAIKDRLDFFYNQGQYEYFQSRVKKNNSTILRMNMIPGPFASNPKIVA LCDAASFPTLFDPSKVSKVNSLTGNYMPALSFTGGYRVCAYLDPSEPTHTKIKQVFFNAQAAKKDTFIPTFVSTF NSMFDKMDAEVESKKKAEFTKFNEAAVFEFVGLALVGPKPAREVFDSAKKSVFFQFHPFITAGLPALVEELAFHM FPFPSFVAKSSYKILYEYFSTGGSWILDNAEEIGLSREEA I HHLIFTWA INAYLGIRTCLMRLFKWIVASGPDLQ EKLAREVRSVVRSEEGKITFAGIEKMELVKSVAYESFRFDP PVQVQYGTAKSDLI IESHDGKYQVKKGEMLCGFQ PMATRDPKVFDRADEFVPDRFMGDGKKLVKHVLWANGYGTDAPKADDKICAGKDLGVLVGRLLIAVMFLRYDKIG GVVGKTMEEVDVIVNELTKVAV*

\subsubsection{BfEAS}

MGNQVGTARLLGWGNQTLKPNREEDFVEKNIGFPCRVVTGNKAVQSVFDIELFKKEEFCFGVAEVRRDFTEGICP SVSSNGKI HEKNKGFLMEVIAKAGEGI PSSTASSVLSNISKWGSTAMSDFESKLLAVAADTVLPNIFGESSSFNA EDIRLYI I GATEVRSCIVKALTSKNLDEERQAMRS I IGKIKTSERYQQLMDLGHAYGLGEAETTAQLLLPVFFNG VAGIRANLVSSFARLDTISAEDREELREEALAALKKHGGLTRESLEEMPKMESFVLEVLRACPSPMFWSTIATRP TTVEYTTDSGEHALKIKEGERVYAS SYWALRDPAVFDKPEDFMWRRFLGPEGDARRKHHVIFHGRLTDTPAVNNH MCPGRDVSLSALKGS IAIFNTFFGWELQEPPVWTGTKMTRGSQPDYEVKIKSFWVQHPEDLKEIFPSHFRDIIDE ADDVDDI DVLVKTKTGTYSGSGTNSNVYIRLFDDKGHQSRELQLDVWWKNDFEKGQEGQYKLKDVKVAAP IVKIE LFRDGCQPDDDWYCESVSVQLNPDNSGPTYDFSVNRWIRQNDHVWLCPGGCEGPQDDVNPKDDLE

\subsubsection{BfEAS_1}

MGSVLGTLQLLGWNNQMLKPNREEDFVEKNIGFPCRVVTGNKTVQSVFDIDLFKKEEFCFGVVGEVRKDFTEGVC PCILSNGKIHEKNKGFLMEVIAKAGEDI PPSTALSVLSNISKWGSTPMSDFESKLTDVAADAFLPNIFGESTHFH GEEIRLYRSGAIAVRLSIVKALTGRNLDEERRAMTSILEKIKTSERYQQLLDLGKSYGLGEKEATAQLLFPVFIN GAYGLAAHLVCTFACLDTISAEDREELREEALAALKNHRGLTRESLEEMPKIES FVLEVLRFCPNPVFWST IATC PTTVEYTTDSGEHTLKIEEGERVYASSYWALRDPAVFDKPEDFMWRRFLGPEGDALRKHHVTFHGRLTDTPAVNN HMCPGKDVSLSALKGS IAIFNTFFGWELQEP PFWTGKKLSRGS LPDNEVKIKSFWVQHPEDLKEIFPSHFQDIVN EVDDVGDI DVLVKTKTGKYSGSGTNSNVYIRLFDDKGHQSRELQLDVWWKDDFEKGQEGQYKLKDIKVAAPIVKI ELFRDGCHPDDDWYCESVSVQLNPDNNGPTYDFPVNRW IRQNDHVWLSPGGGEPPKDDVNPIDD

\subsubsection{BfEAS_2}

MGAGSVVGFDLTSCNIALDLI PVVEGLISKRKRNPFWYVWDDKGSEIFEKIATTSATYTLWKREFTLLQRKCNEI ASKTTSPTILVELGSGASSKTRL I IEAMLKQHGSLTFVPVDIAKGRI IMGNALGYLKWLKSNNQAFAPDRHEDFV ETNIGFPCRVVTGNKAVQSVFDVDLFRKEEFYFGP IEVRKDFTEGVCPSITSNGKIHERNKALMMDI IAEAYKEI PTSTANAVLSNIARWGRRPVDFESKLFAVSAGALLPTIFGKTTHFNAEEIELYISGSTELRPDI LAALLEGKDLA EGRPAMAYVLEKMKTSERYHQLIDLGKS HGFGEKETTGQLLFS IMFNGVGGMAVNLVPS FAHLDTISAEDREELR EEALAALKKHGGLTREALAEMPKIESFVLEVLRACPAPDFWSTIATRPTTVKYSTESGPQEVEIKEGERVYASSY WALRDPAVFDKPDNFMWRRFLGPEDKARREHHVTFHGRLIDTPATNNHMCPGKDVGLSVIKGS IALLNTFFGWEL EEPPVWTGTKAARLGQPDNEVNINTFWLQHPNDLKAVFPSYFDDI I DGLNKNPEDSDVAGIDVLVKTKTGKYRGS GTNSNVYIRLYDDKGHQSRELKLDVWWKNDFERGQKGQYKLKDVKVAAPIVKIELFRDGCHPDDDWYCESVSVQL NPDKKGPTYDFPVNRWIRQNDHVWLSAGGCEPTKDDMKPKDG 


\subsection{Alignment}

AtAOS

PaAOS

LeAOS 3

PpAOS1

PpAOS2

AtHPL

LeHPL

PpHPL

StDES

AsDes

BfEAS

BfEAS 1

BfEAS_2

AtAOS

PaAOS

LeAOS3

PpAOS1

PpAOS2

AtHPL

LeHPL

PpHPL

StDES

AsDes

BfEAS

BEEAS 1

BfEAS_2

AtAOS

PaAOS

LeAOS 3

PpAOS 1

PpAOS2

At HPL

LeHPL

PpHPL

StDES

AsDes

BfEAS

BfEAS_1

BfEAS_2

AtAos

PaAOS

LeAOS3

PpAOS1

PpAOS2

AtHPL

LeHPL

PpHPL

StDES

AsDes

BEEAS

BfEAS_1

BfEAS_2

AtAos

PaAOS

LeAOS 3

PpAOS 1

PpAOS2

AtHPL

LeHPL

PpHPL

StDES

AsDes

BEEAS

BfEAS 1

BfEAS_2

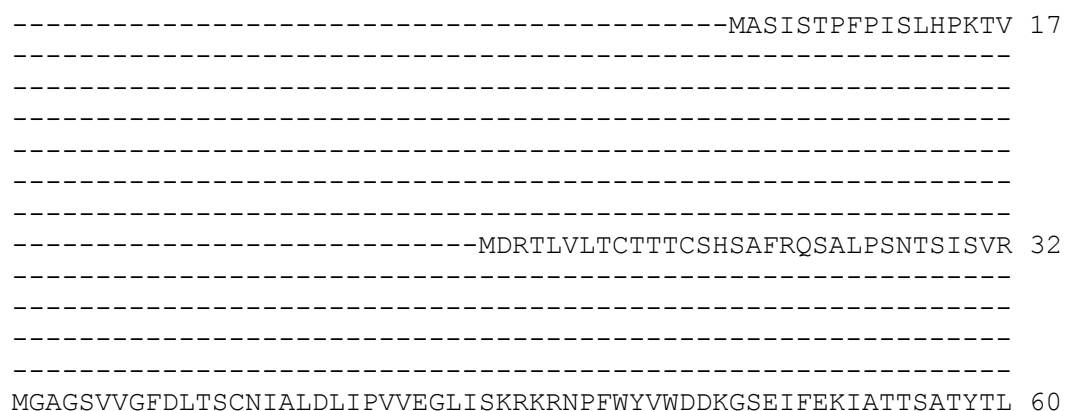

RSKPLKFRVLTRPIKASGSETPDLTVATRTGSKDLPIRNIPGNYGLPIVGPIKDRWDYFY 77 -MDPSSK--PLREIPGSYGIPFFQPIKDRLEYFY 31 ----------MANTKDSYHIITMDTKESS I PSLPMKEI PGDYGVPFFGAIKDRYDFHY 48

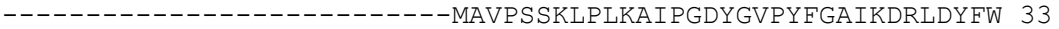
------------------------MAVPVSNLPLRAIPGGYGISYLGAIKDRLDYFW 33 --------------------------------------------------------

----------------MNSAPLSTPAPVTLPVRSIPGSYGLPLVGPIADRLDYFW 39 LGTCSVRTQKRRTVVASLGNIETTSTSTVGQESNLPLREIPGSYGIPYLSQLLDRWTFFY 92 $-------------n-----M S S-Y S E L S N L P I R E I$ PGDYGFPIISAIKDRYDYFY 35 -------------------MSTSNGSTENIQKPLRKI PDITGTPILTAIKDRLDFFY 38

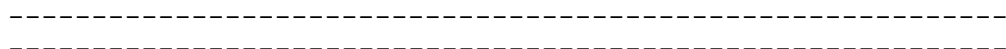
WKREFTLLQRKCNEIASKTTSPTILVELGSGASSKTRLI IEAMLKQHGSLTFVPVDIAKG 120

DQG-AEEFFKSRIRKYNSTVYRVNMPP--GAFIAENPQVVALLDGKSFPVLFDVDKVEKK 134 GTGGRDEYFRSRMQKYQSTVFRANMPP--GPFVSSNPKVIVLLDAKSFPILFDVSKVEKK 89 NQG-ADEFFRSRMKKYDSTVFRTNVPP--GPFNARNSKVVVLVDAVSYPILFDNSQVDKE 105 LQG-EEQFYRSRMAKYNSTVFRVNMPP--GPPISEHPQVICLLDQKSFPILFDVSKVEKK 90 IQG-EEEFYRSRVEKYNSTVFRVSMPP--GPPIAKDARVICVLDQKSFPILFDVNKCEKR 90 FQK-PENFFTKRMEKHKSTVFRTNVPPCFPFFGSVNPNVVAVLDVKSFSHLFDMEIVEKA 98 REG-EPQFWQSRMAKYGSTVIRSNMPP--GWFWT-DSRCIMLLDQKSYPTVFDYDKVDKY 148 NQG-EDAWFHNKAEKYKSTVVKINMAP--GPFTSNDYKLVAFLDANSFVCMFDNSLIDKT 92 NQG-QYEYFQSRVKKNNSTILRMNMI P--GPFAS-NPKIVALCDAASFPTLFDPSKVSKV 94 ---MGNQVGTARLLGWGNQTLKPNREEDFVEKNIGFPCRVVTGNKAVQSVFD--IELFKK 55 ---MGSVLGTLQLLGWNNQMLKPNREEDFVEKNIGFPCRVVTGNKTVQSVFD--IDLFKK 55 RI IMGNALGYLKWLKSNNQAFAPDRHEDFVETNIGFPCRVVTGNKAVQSVFD--VDLFRK 178

DLFTGTYMPSTELTGGYRILSYLDPSEPKHEKLKNLLFFLLKSSRNRIFPEFQATYSE-L 193 DLFTGTYMPSTKLTGAYRVLSYLDPSEPRHAQLKNLLFFMLKNSSNRVIPQFETTYTE-L 148 NYFEGTFMSSPSFNGGYKVCGFLGTSDPKHTTLKGLFLSTLTRLHDKFIPIFTTSITS-M 164 DVFTGTYMPSVSFTSGYRVCSYLDPSEERHTKLKQWCFEVIAMNGRNFLPEFHKSIEE-S 149 DLFLGTYMPDLSYTSGHRVLSYLDPSEVRHEKLKQWCFDLIARNGRKFLPEFHTAMEE-S 149 DVLIGDFRPSLGFYGGVRVGVYLDTTEPKHAKIKGFAMETLKRSSKVWLQELRSNLNI -F 66 NVLVGDFMPSVVYTGDMRVCAYLDTSEPKHAQIKNFSQDILKRGSKTWVPTLLKELDT-M 157 KAFAGTIMPSTEYNGGYEVCAYLDASDKKHEQLKGYCFELLKFSSSKWAREFHTAISE-T 207 DTLGGTFKPGKEYYSGYRPVAFIDTKDPNHAALKGYILSAFAKRHNLFIPLFRNSLSDHL 152 NSLTGNYMPALSFTGGYRVCAYLDPSEPTHTKIKQVFFNAQAAKKDTFIPTFVSTFNS-M 153 EEFCFGVA-EVRRDFTEGICPSVSSNGKIHEKNKGFLMEVIAKAGEGIPSSTASSVLS-- 112 EEFCFGVVGEVRKDFTEGVCPCILSNGKIHEKNKGFLMEVIAKAGEDIPPSTALSVLS-- 113 EEFYFGPI-EVRKDFTEGVCPS ITSNGKIHERNKALMMDI IAEAYKEIPTSTANAVLS-- 235

FDSLEKELSLKGKADFGGSSDGTAFNFLARAFYG---TNPADTKLKADAPGLITKWVLFN 250 FEGLEAELAKNGKAAFNDVGEQAAFRFLGRAYFN---SNPEETKLGTSAPTLISSWVLFN 205 FTSLEKELSEKGTSYFNPIGDNLSFEFLFRLFCEG--KNPIDTSVGPNGPKIVDKWVFLQ 222 MVLWETSLAKGEKTSVSDEVKOFAFNFLMRAVCHHDPAAPGEYSLGRNGGPYATAWANPQ 209 FAVWEEAMEKGENANLSEEVQQFAFNFLVRAVLHHDPVAPGEASLGKNGGPYASAWHGPQ 209 WGTIESEISKNGAASYIFPLQRCIFSFLCASLAG--VDASVSPDIAENGWKTINTWLALQ 124 FTTFEADLSKSNTASLLPALQKFLFNFFSLTILG--ADPSVSPEIANSGYIFLDSWLAIQ 215 FNQWEGKLAQKTPALINPTLPESLFSFVINALTTARFDDSS IPDAEKPVCGDLQKWAGFQ 267 FNNLEKQVTEQGKSDFNALLPTMTFNFIFRLLCDQ--TNPSDTVLGAQGPEHLRKWLFPQ 210 FDKMDAEVESKKKAEFTKFNEAAVFEFVGLALVG---PKPAREVFDS-----AKKSVFFQ 205 --NISKWGSTAMSDFESKLLAVAADTVLPNIFGESSSFNAEDIRLYIIGATEVRSCIVKA 170 --NISKWGSTPMSDFESKLTDVAADAFLPNIFGESTHFHGEEIRLYRSGAIAVRLSIVKA 171 --NIARWGRRPV-DFESKLFAVSAGALLPTIFGKTTHFNAEEIELYISGSTELRPDILAA 292 
AtAOS

PaAOS

LeAOS 3

PpAOS1

PpAOS2

AtHPL

LeHPL

PpHPL

StDES

AsDes

BIEAS

BEEAS 1

BfEAS ${ }^{-} 2$

AtAOS

PaAOS

LeAOS3

PpAOS 1

PpAOS2

AtHPL

LeHPL

PpHPL

StDES

AsDes

BfEAS

BfEAS 1

BfEAS ${ }^{-} 2$

AtAOS

PaAOS

LeAOS 3

PpAOS 1

PpAOS2

AtHPL

LeHPL

PpHPL

StDES

AsDes

BIEAS

BEEAS 1

BfEAS ${ }^{-2}$

AtAOS

PaAOS

LeAOS3

PpAOS 1

PpAOS2

AtHPL

LeHPL

PpHPL

StDES

AsDes

BEEAS

BfEAS 1

BfEAS ${ }^{-} 2$

AtAOS

PaAOS

LeAOS 3

PpAOS1

PpAOS2

AtHPL

LeHPL

PpHPL

StDES

AsDes

BEEAS

BfEAS_1

BfEAS ${ }^{-}$
LHP--LLSIG-LPRVIEEPLIHTFSLPPALVKSDYQRLYEFFLESAGEILVEADK-LGIS 306 LAP--TLDLG-LPWFLQEPLLHTFRLPAFLIKSTYNKLYDYFQSVATPVMEQAEK-LGVP 261 LAPLISLGLKFVPNFLEDLVLHTFPLPYILVKRDHQKLYNAFYNSMKDILDEAEK-LGVK 281 LAP--IAGQTGLPHVVEELVLHTVPLPSALVKKNYDALYNFIKNYATEALDRAEA-MGIE 266 LAP--IAGQTGLPHAVEEL-LHTIRLPSSVVKEQYDALYNFFKTYGGEELDRAVA-LGIK 265 VIP--TAKLGVVPQPLEEILLHTWPYPSLLIAGNYKKLYNFIDENAGDCLRLGQEEFGLT 182 LAP--TVSIGVL-QPLEEILVHSFAYPFFLVKGNYEKLVQFVKNEAKEVLSRAQTGFQLT 272 LMP---VIRTGAPIYIEEM-LHVAPIPASLTKGGYDKMVVFLQKYAAETLSIAEK-FGLS 322 LIP--SLSAKKLPNI IEDTLFHNFLIPFGFIKSDYNKLVDAFSKSAVSILDEAEK-LGIK 267 FHP--FITAG-LPALVEELAFHMFPFPSFVAKSSYKILYEYFSTGGSWILDNAEE-IGLS 261 LT---------SKNLDEERQAMRS I I GKIKTSERYQQLMDLGHAYG------------LG 209 LT--------GRNLDEERRAMTS ILEKIKTSERYQQLLDLGKSYG-----------LG 210 LLE--------GKDLAEGRPAMAYVLEKMKTSERYHQLIDLGKSHG------------FG 332

REEATHNLLFATCFNTWGGMKILFPNMV-KRIGRAGHQVHNRLAEEIRSVIKSNG-GELT 364 KDEAVHNILFAVCFNTFGGVKILFPNTL-KWIGVAGENLHTQLAEEIRGAIKSYGDGNVT 320 RDEACHNFVFLAGFNSYGGLKVFFPSLI-KWIGTSGPSLHARLVKEIRTAVKEAG-G-VT 338 RNDATANLLFFLCFNAYGGFSIFFPLIT-ILISSCGPELMHDLHDEVTKAVAATD-GKVT 324 RDDAIANLLFLLGFNAYGGFNFFFPQLT-GHIAQCGPELMHELHEEVVAAVQATE-GKVT 323 RDEAIQNLLFVLGFNAYGGFSVFLPSLIGRITGD-NSGLQERIRTEVRRVCGSGS-D-LN 239 EQEAIHNLLFILGFNAFGGFS IFLPTLLGNLGDEKNADMQEKLRKEVRDKVGVNP-ENLS 331 QDEAVHNLIFFLILNAHGGFCRFLPVIL-REVAKNG-QLQADLREEVRAAVKASGSDQVT 380 REEAVQNILFLVGINMFAGLNAFSPHLF-RFVGEAGASLHTQLAKEIRTVIKEEG-GAIT 325 REEAIHHLIFTWAINAYLGIRTCLMRLF-KWIVASGPDLQEKLAREVRSVVRSEE-GKIT 319 EAETTAOLLLPVFFNGVAGIRANLVSSF-ARLDTISAEDREELREEALAALKKHG--GLT 266 EKEATAQLLFPVFINGAYGLAAHLVCTF-ACLDTISAEDREELREEALAALKNHR--GLT 267 EKETTGQLLFSIMFNGVGGMAVNLVPSF-AHLDTISAEDREELREEALAALKKHG--GLT 389

MGAIEK-MELTKS-----VVYECLREEPPVTAQYG-----RAKKDLVIESHDAA------ 407 LEAIEQ-MPLTKS-----VVYESLRIEPPVPPQYG-----KAKSNFTIESHDAT------ 363 LSAIDK-MPLVKS-----VVYETLRMDP PVPFQTV-----KARKNIIITNHESS------ 381 LQSIEN-MPLVKS-----VVYEAFRFKPPVPYQYG-----KAKFDFTIENHENS------ 367 PKSLEN-MPLLSS-----VVYEGFRMKPPVPYQYA-----RAKTDFLIESHENS------ 366 FKTVNE-MELVKS-----VVYETLRFSPPVPLQFA-----RARKDFQISSHDAV------ 282 FESVKE-MELVQS-----FVYETLRLSPPVPSQYA-----RARKDFKLSSHDSV------ 374 MKAVMNDMPLVAS-----TVFEALRFDPPVPFQYA-----RAKKDFIIESHDAR------ 424 LSAINK-MSLVKS-----VVYETLRLRPPVPLQYG-----KAKKDFMVQSHDAS------ 368 FAGIEK-MELVKS-----VAYESFRFDPPVQVQYG-----TAKSDLIIESHDGK------ 362 RESLEE-MPKMES-----FVLEVLRACPSPMFWST-IATRPTTVEYTTDSGEHA------ 313 RESLEE-MPKIES-----FVLEVLRFCPNPVFWST-IATCPTTVEYTTDSGEHT------ 314 REALAE-MPKIES-----FVLEVLRACPAPDFWST-IATRPTTVKYSTESGPQE------ 436

FKVKAGEMLYGYQPLATRDPKIFD-RADEFVPERFVGEEGEKLLRHVLWSNGPETETPTV 466 FEVKKGEMLFGYQPFATKDPKVFD-RPEEFVPDRFVGD-GEALLKYVWWSNGPETESPTV 421 FLIKKDELIFGYQPLATKDSKVFK-NAEEFNPDRFVGG-GEKLLKYVYWSNGKEIDNPSV 439 FEVKKGEMLYGYOPIVMHDPKVFS-DPDOFLPRRFMGPDGEKLIKYIFWSNGYETDEPTT 426 FEVKKGEMLYGFQPYVMHDPNVFE-NPDKFLPRRFMGPEGEALLGNVFWSNGRETDDPTV 425 FEVKKGELLCGYQPLVMRDANVED-EPEEFKPDRYVGETGSELLNYLYWSNGPQTGTPSA 341 YEIKKGELLCGYQPLVMKDPKVFD-EPEKFVLERFTKEKGKELLNYLFWSNGPQTGRPTE 433 YQIKTGDFLGGVNYMVSRDPKVFTDRPNEFNARRFMGPEGDKLLAHLVWSNGRQTDETTV 484 YKINKGQFVVGYQPMASRDPKIFA-NPDEFVPDRFMND-GEKMLKHVLWSNGRETENPAP 426 YQVKKGEMLCGFQPMATRDPKVFD-RADEFVPDRFMGD-GKKLVKHVLWANGYGTDAPKA 420 LKIKEGERVYASSYWALRDPAVFD-KPEDFMWRRFLGPEGDARRKHHVIFHGRLTDTPAV 372 LKIEEGERVYASSYWALRDPAVFD-KPEDFMWRRFLGPEGDALRKHHVTFHGRLTDTPAV 373 VEIKEGERVYASSYWALRDPAVFD-KPDNFMWRRFLGPEDKARREHHVTFHGRLIDTPAT 495

GNKQCAGK--DFVVLVARLFVIEIFRRYDSFDIEVGTSPLGSSVNFSSLRKASF------ 518 ENKQCAGK--DFVVLITRLFVIELFRRYDSFEIELGESPLGAAVTLTFLKRASI------ 473 NDKQCPGK--DLIVLMGRLLVVEFFMRYDTFEVEFGKLLLGSKVTFKSLTKATS------ 491 ANKQCAGK--DLVVTMARAFVAEMFLRYKEYTLTMEGAGNATKVFFSDLKK--------- 475 HDKQCAGK--DLAVTISRAYVAEMFLRYKEFTLEVQGSGVQTTLLFSALQKA-------- 475 SNKQCAAK--DIVTLTASLLVADLFLRYDTITGDSG--------SIKAVVKAK------- 384 SNKQCAAK--DMVTLTASLIVAYIFQKYDSVSFSSG--------SLTSVKKAS------- 476 YTKQCAGK--EIVPLTGRLLLAELFMRFDSFNIEG----LEMEATFTSLTPRSD------ 532 DNKQCPGK--DLVHLLGRLILVEFFMRYDTFTVEITPLFRAPNVAFKTLTKASK------ 478 DDKICAGK--DLGVLVGRLLIAVMFLRYDKIGGVVGKTMEEVDVIVNELTKVAV------ 472 NNHMCPGRDVSLSALKGSIAIFNTFFGWELQEPPVWTGTKMTRGSQPDYEVKIKSFWVQH 432 NNHMCPGKDVSLSALKGSIAIFNTFFGWELQEPPFWTGKKLSRGSLPDNEVKIKSFWVQH 433 NNHMCPGKDVGLSVIKGS IALLNTFFGWELEEPPVWTGTKAARLGQPDNEVNINTFWLQH 555 


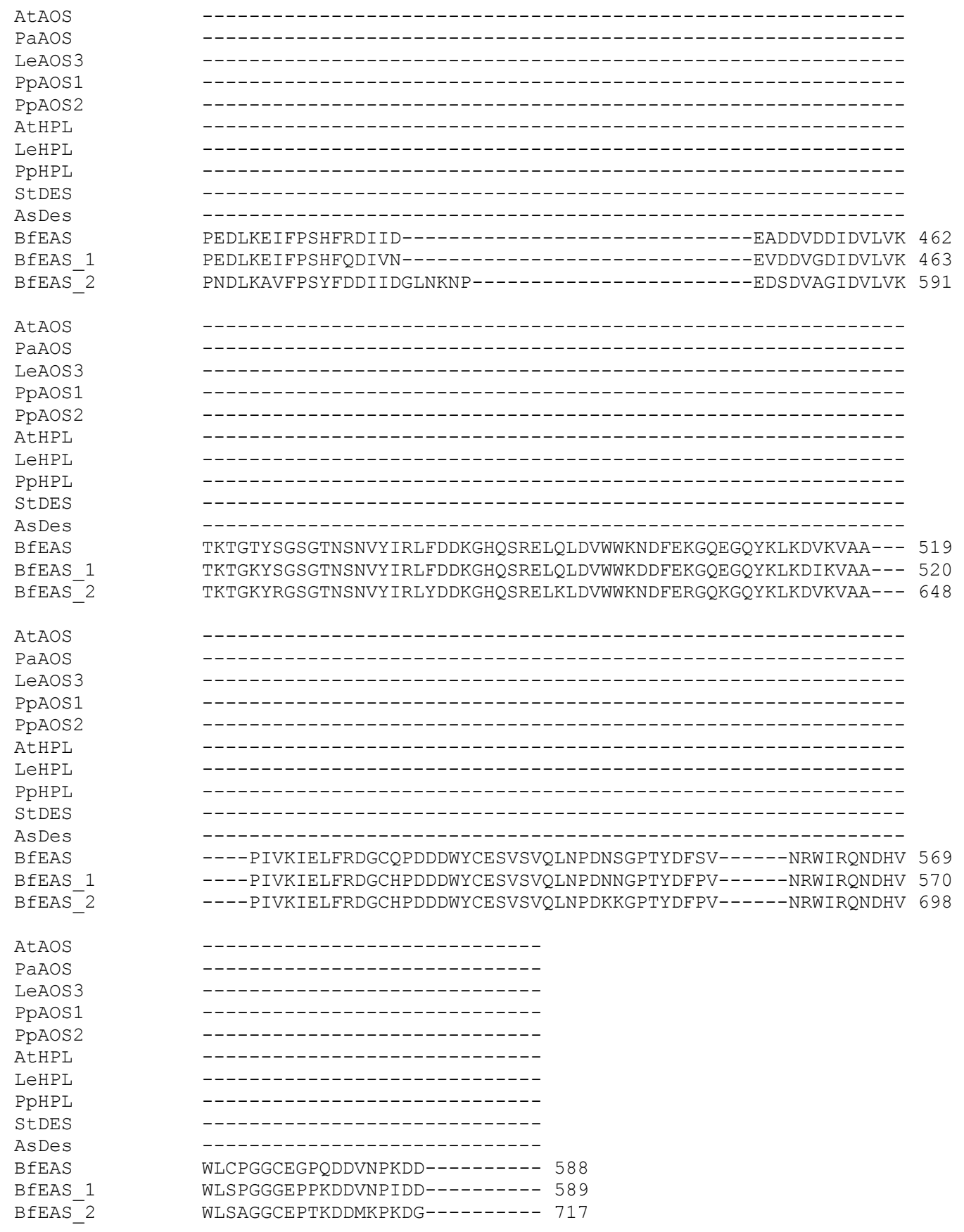




\subsection{Primer}

\subsubsection{Mutagenesis Primer}

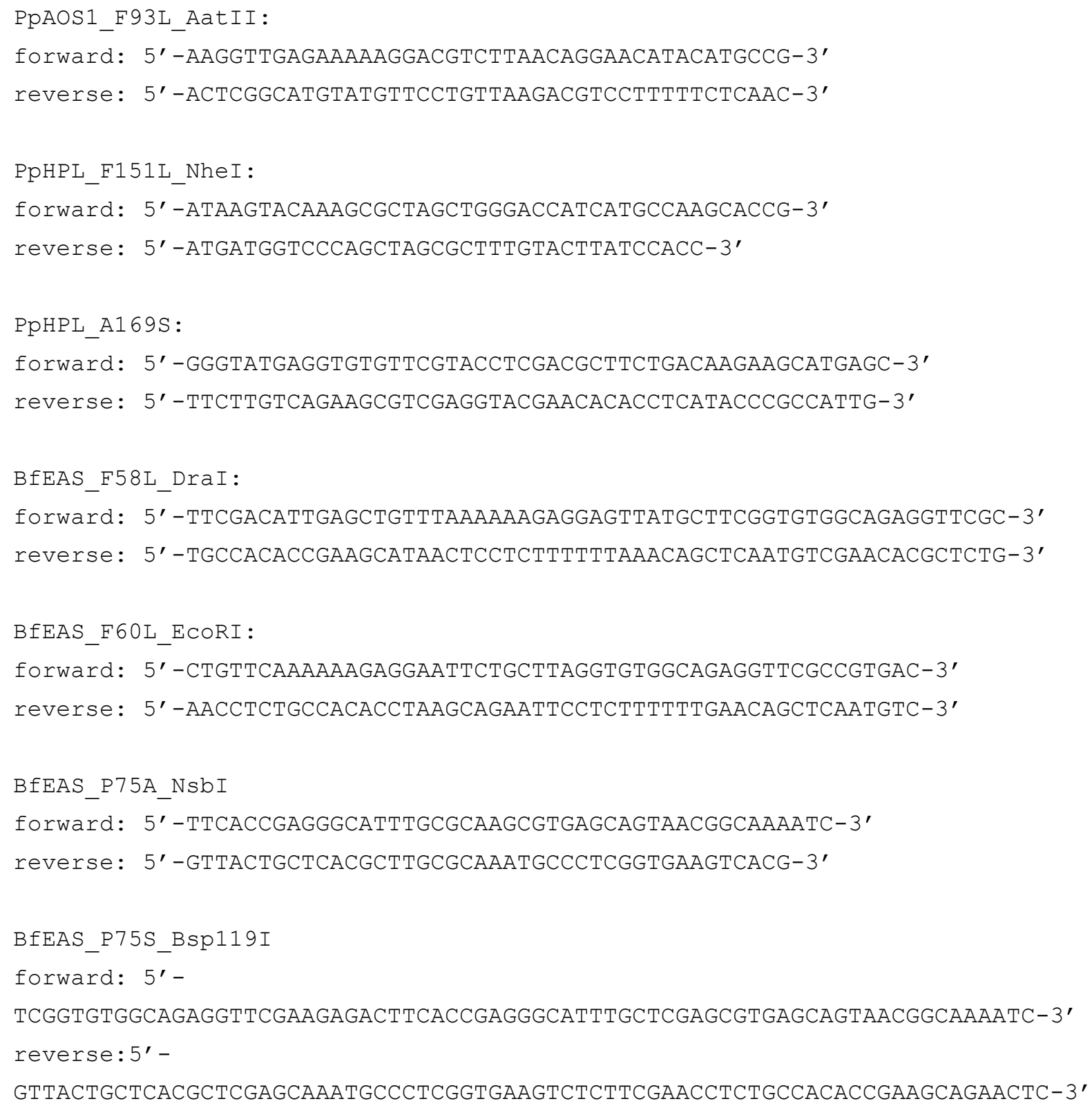


BfEAS_F223I_PdiI

forward: 5'-CTGTTACCGGTGTTTATCAACGGCGTTGCCGGCATTCGTGCAAACCTGGTGAGC-3' reverse: 5'-CAGGTTTGCACGAATGCCGGCAACGCCGTTGATAAACACCGGTAACAGCAGCTG-3'

BfEAS_F223L_PdiI

forward: 5'-CTGTTACCGGTGTTTTTAAACGGCGTTGCCGGCATTCGTGCAAACCTGGTGAGC-3'

reverse: 5'-CAGGTTTGCACGAATGCCGGCAACGCCGTTTAAAAACACCGGTAACAGCAGCTG-3'

BfEAS_N224Q_PdiI

forward: 5'-TTACCGGTGTTTTTCCAGGGCGTTGCCGGCATTCGTGCAAACCTGGTGAGC-3'

reverse: 5'-CAGGTTTGCACGAATGCCGGCAACGCCCTGGAAAAACACCGGTAACAGCAG-3'

BfEAS_9AS-deletion:

forward: $5^{\prime}-$

CGTCGCAAACACCACGTTATCTTCCATGGCAATCACATGTGTCCGGGTCGTGACGTTAGTCTGAGC-3' reverse: $5^{\prime}-$

ACTAACGTCACGACCCGGACACATGTGATTGCCATGGAAGATAACGTGGTGTTTGCGACGTGCGTC-3' 


\subsection{Additional Figures}

\subsubsection{Biochemical characterization of CYP74-enzymes from P. patens}

\subsubsection{Analysis of products(wild types)}
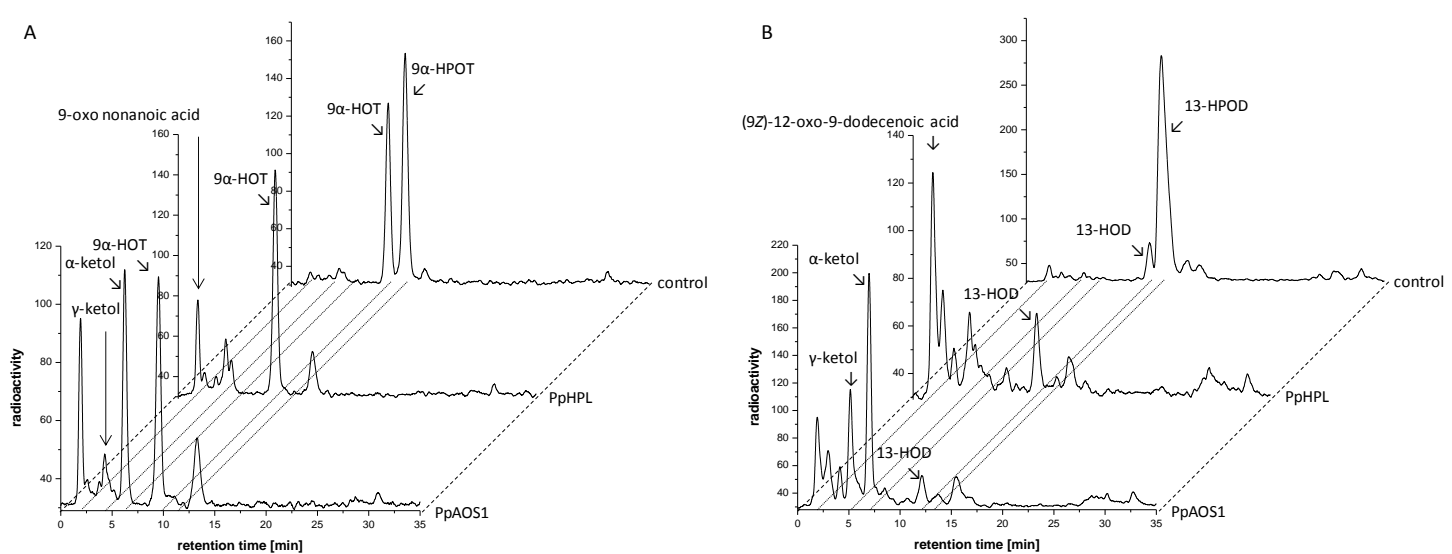

Figure 9.1: Product analyses from incubation of PpAOS1 and PpHPL with $\left[1-{ }^{14} \mathrm{C}\right]-9 \alpha-\mathrm{HPOT}(\mathrm{A})$ andwith $\left[1-{ }^{14} \mathrm{C}\right]-$ 13-HPOD (B). The purified enzymes as well as only buffer (control) were incubated with the radio-labeled substrate for approx. $30 \mathrm{~min}$ at RT. After extraction with diethyl ether the products were analyzed by RP-HPLC coupled to a radio-detector. The chromatograms shown are representative for three to four(A) and three to five (B) experiments.

\subsubsection{Analysis of products (site-directed mutagenesis)}
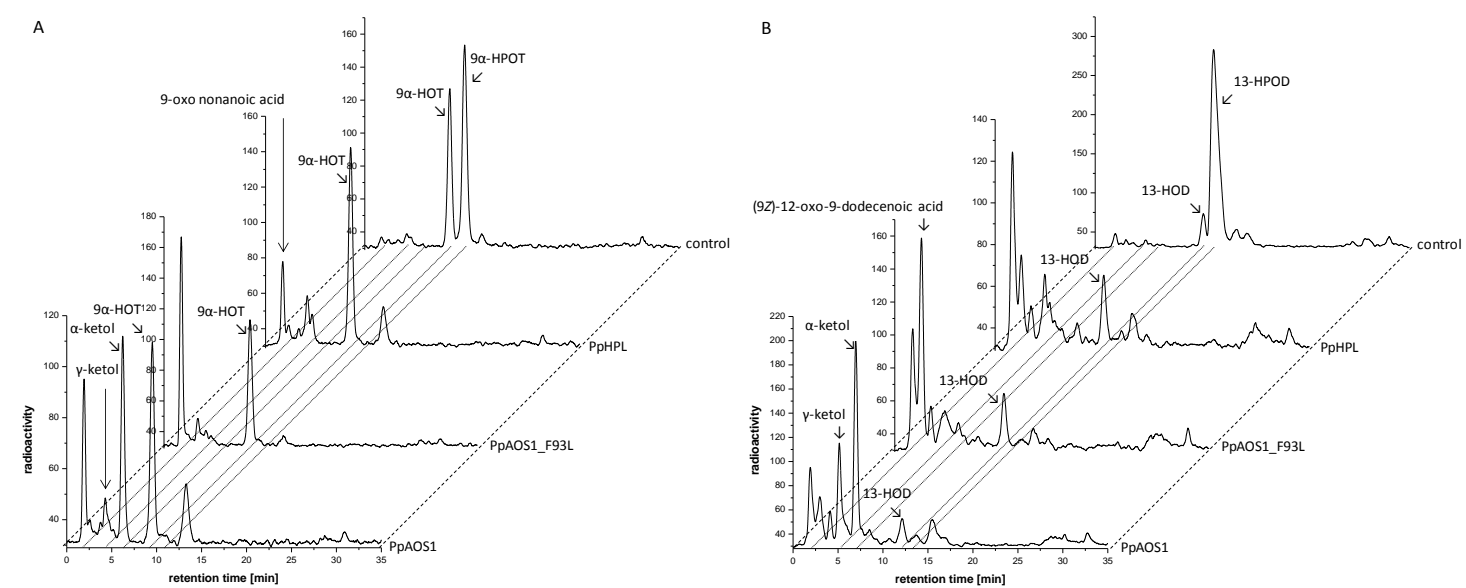

Figure 9.2: Product analyses of PpAOS_F93L with $\left[1-{ }^{14} \mathrm{C}\right]-9 \alpha-\mathrm{HPOT}$ (A) andwith $\left[1-{ }^{14} \mathrm{C}\right]-13-\mathrm{HPOD}$ (B) in comparison to the PpAOS1 and PpHPL wild types. The purified enzymes as well as only buffer (control) were incubated with the radio-labeled substrate for approx. $30 \mathrm{~min}$ at RT. After extraction with diethyl ether the products were analyzed by RP-HPLC coupled to a radio-detector. The chromatograms shown are representative for two to four(A) and two to five (B) experiments. 


\subsubsection{Biochemical characterization of BfEAS}

\subsubsection{Analysis of products (wild type)}

A

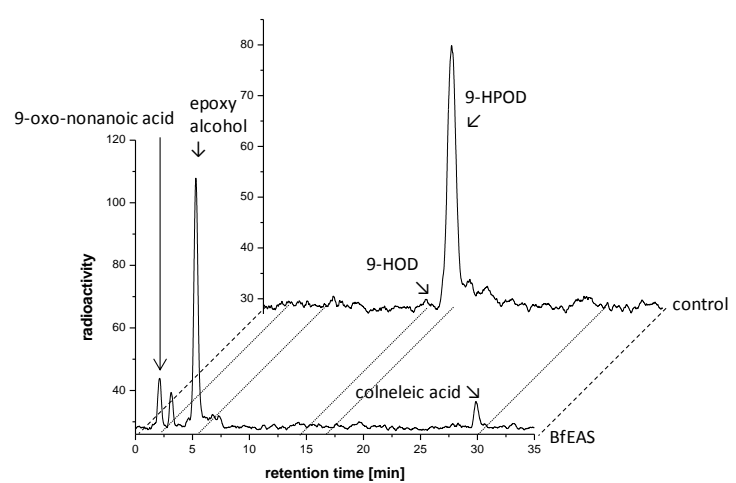

B

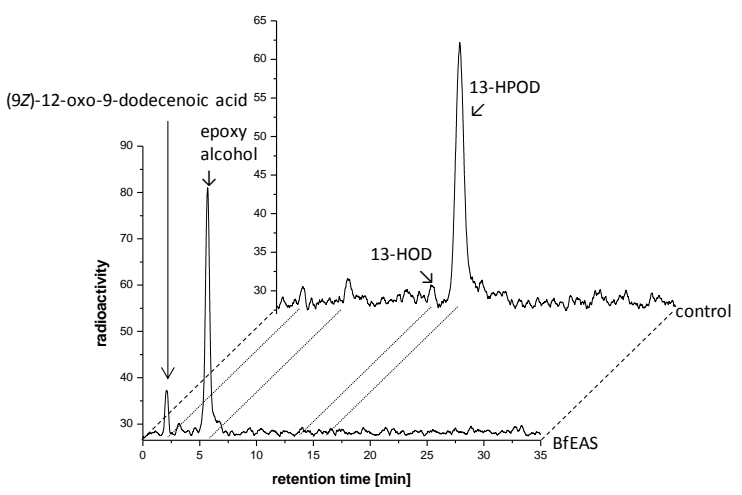

Figure 9.3: Product analyses from incubation of BfEAS with $\left[1-{ }^{14} \mathrm{C}\right]-9-\mathrm{HPOD}(\mathrm{A})$ and with $\left[1-{ }^{14} \mathrm{C}\right]-13-\mathrm{HPOD}(\mathrm{B})$. The purified enzyme was incubated with the radio-labeled substrate for approx. 30 min at RT. After extraction with diethyl ether the products were analyzed by RP-HPLC coupled to a radio-detector. The chromatograms shown are representative for three $(A)$ and for five $(B)$ experiments.

\subsubsection{Analysis of products (site-directed mutagenesis)}

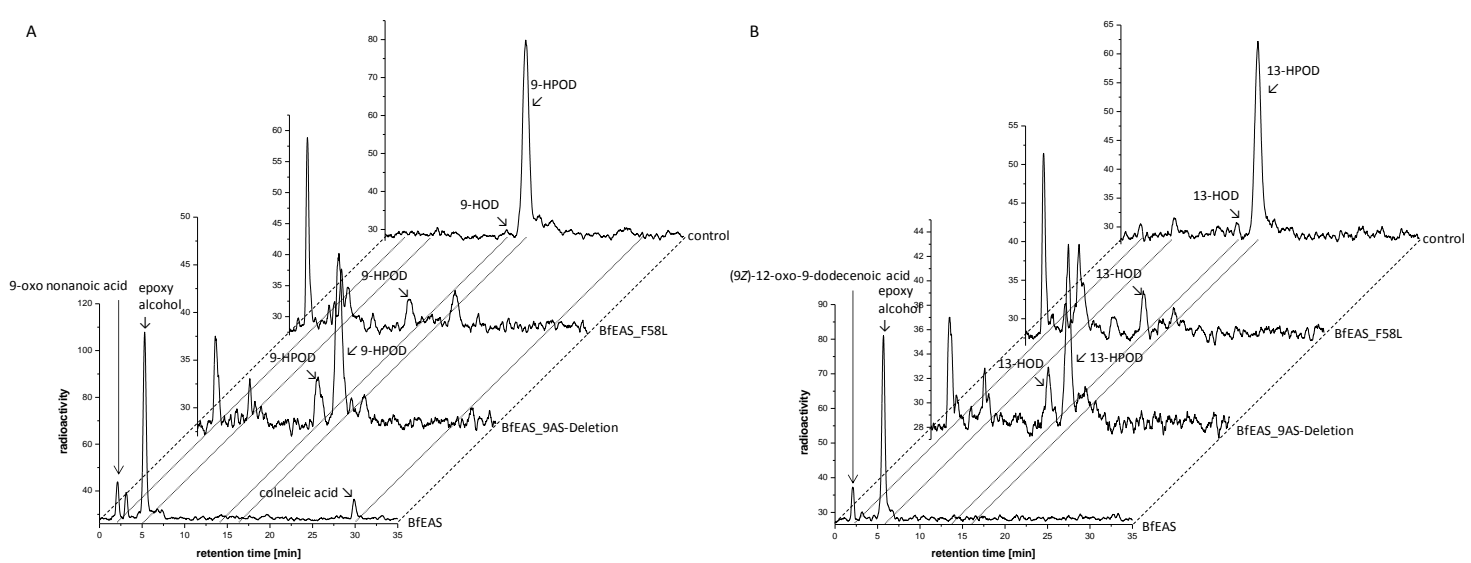

Figure 9.4:Product analyses from incubation of BfEAS_F58L and BfEAS_9AS-deletion with $\left[1-{ }^{14} \mathrm{C}\right]-9-\mathrm{HPOD}$ (A) and with $\left[1-{ }^{14} \mathrm{C}\right]-13-\mathrm{HPOD}(\mathrm{B})$ in comparison to BfEAS wild type. The purified enzymes were incubated with the radio-labeled substrate for approx. $30 \mathrm{~min}$ at $\mathrm{RT}$. After extraction with diethyl ether the products were analyzed by RP-HPLC coupled to a radio-detector. The chromatograms shown are representative for two to three (A) and two to five (B) experiments. 
A

B

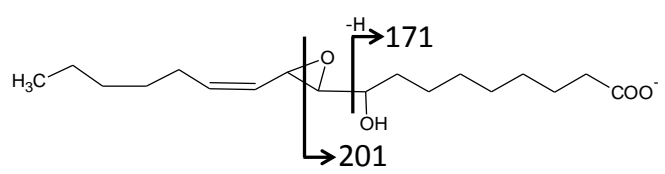

(12Z)-9-hydroxy-10,11-epoxy-12-octadecenoic acid
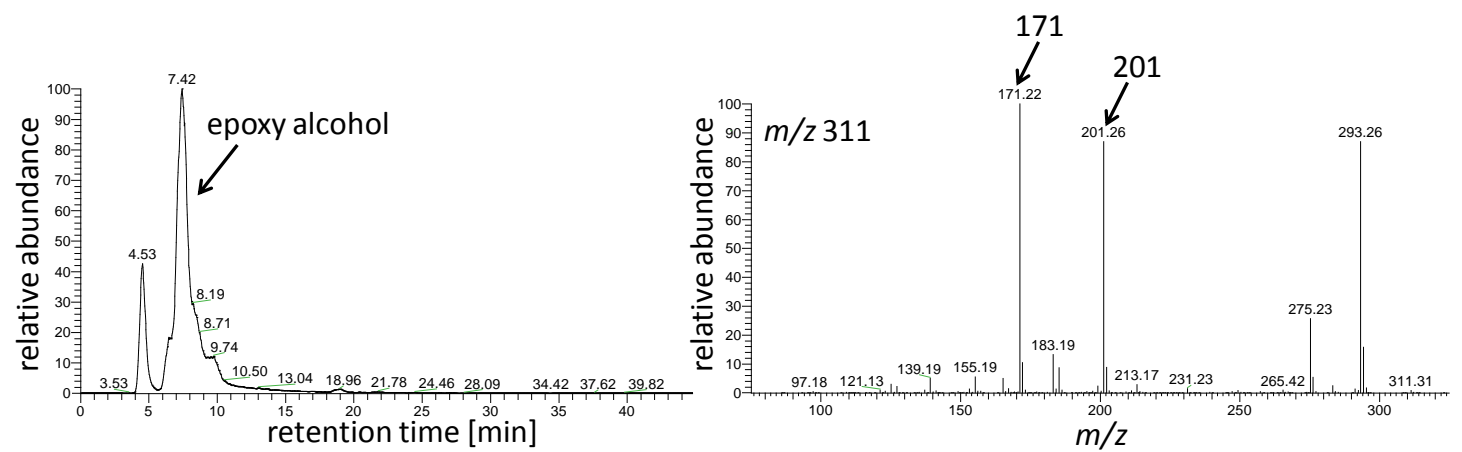

Figure 9.5: RP-HPLC/MS ${ }^{2}$-analysis of products formed by incubation of BfEAS with 9-HPOD. Shown are the extracted ion chromatograms $(\mathrm{m} / \mathrm{z} 311)(\mathrm{A})$ and the associated tandem mass spectrum (B).

A

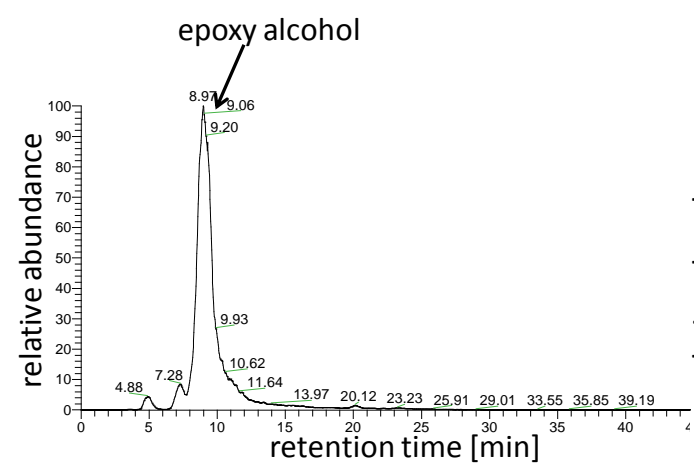

B

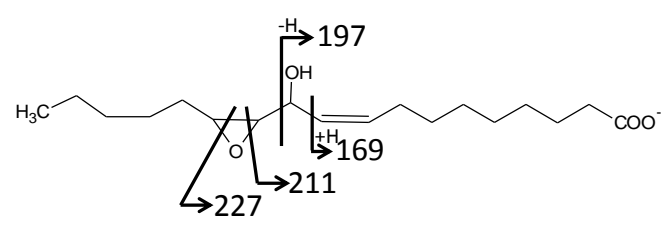

(9Z)-11-hydroxy-12,13-epoxy-9-octadecenoic acid

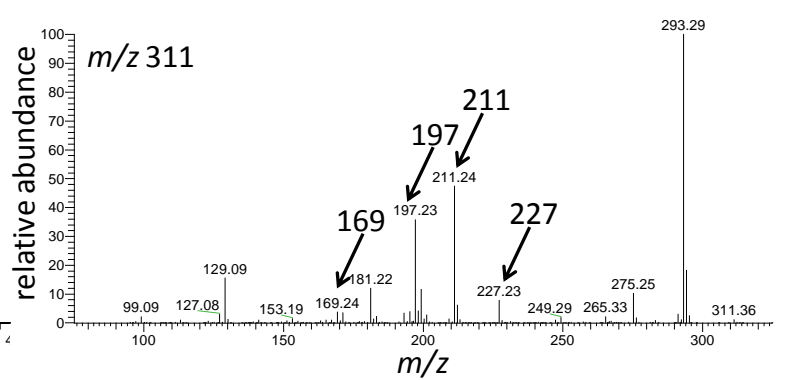

Figure 9.6: RP-HPLC/MS ${ }^{2}$-analysis of products formed by incubation of BfEAS with 13-HPOD. Shown are the extracted ion chromatograms $(\mathrm{m} / \mathrm{z} 311)(\mathrm{A})$ and the associated tandem mass spectrum (B).

A

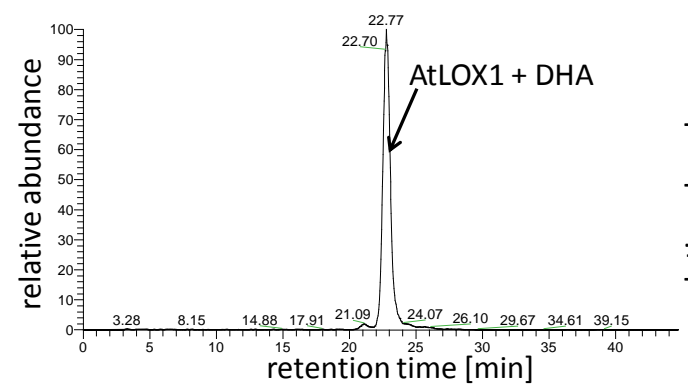

B
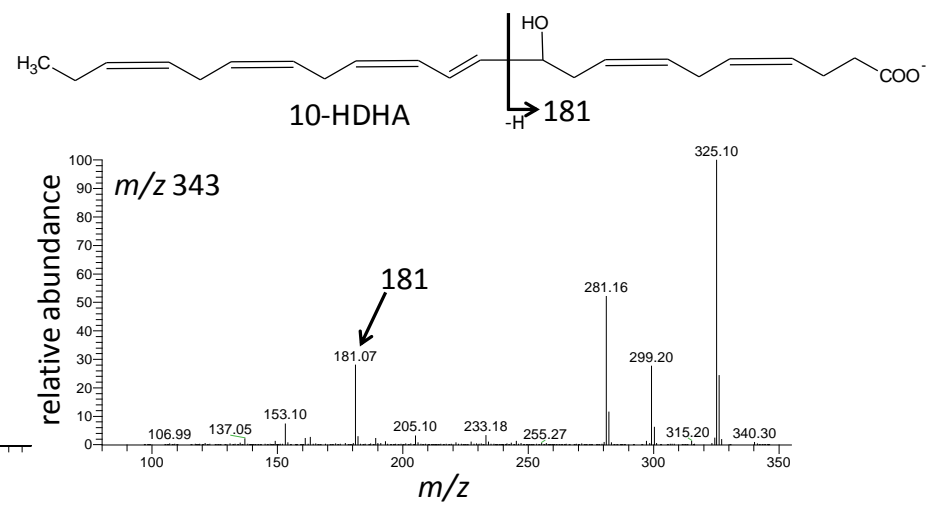

Figure 9.7: RP-HPLC/MS ${ }^{2}$-analysis of products formed by incubation of AtLOX1 with DHA. Shown are the extracted ion chromatograms $\mathrm{m} / \mathrm{z} 343$ and the associated tandem mass spectrum of 10-HDHA. 
A

B
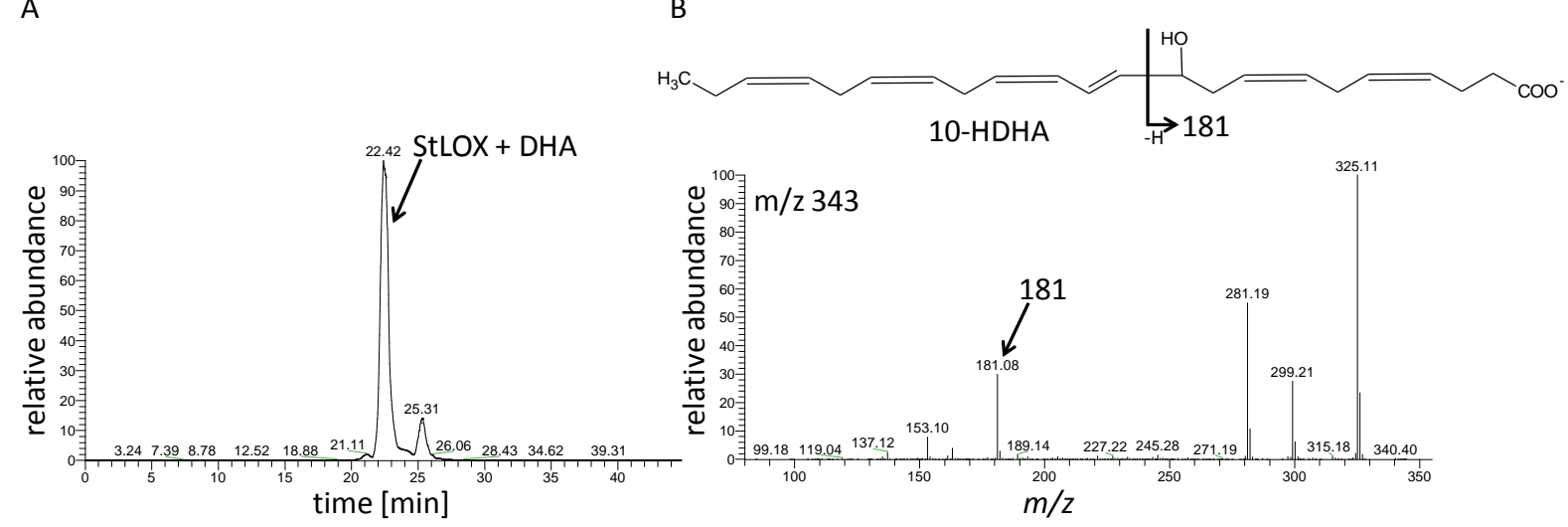

Figure 9.8: RP-HPLC/MS ${ }^{2}$-analysis of products formed by incubation of StLOX with DHA. Shown are the extracted ion chromatograms $\mathrm{m} / \mathrm{z} 343$ and the associated tandem mass spectrum of 10-HDHA.

A
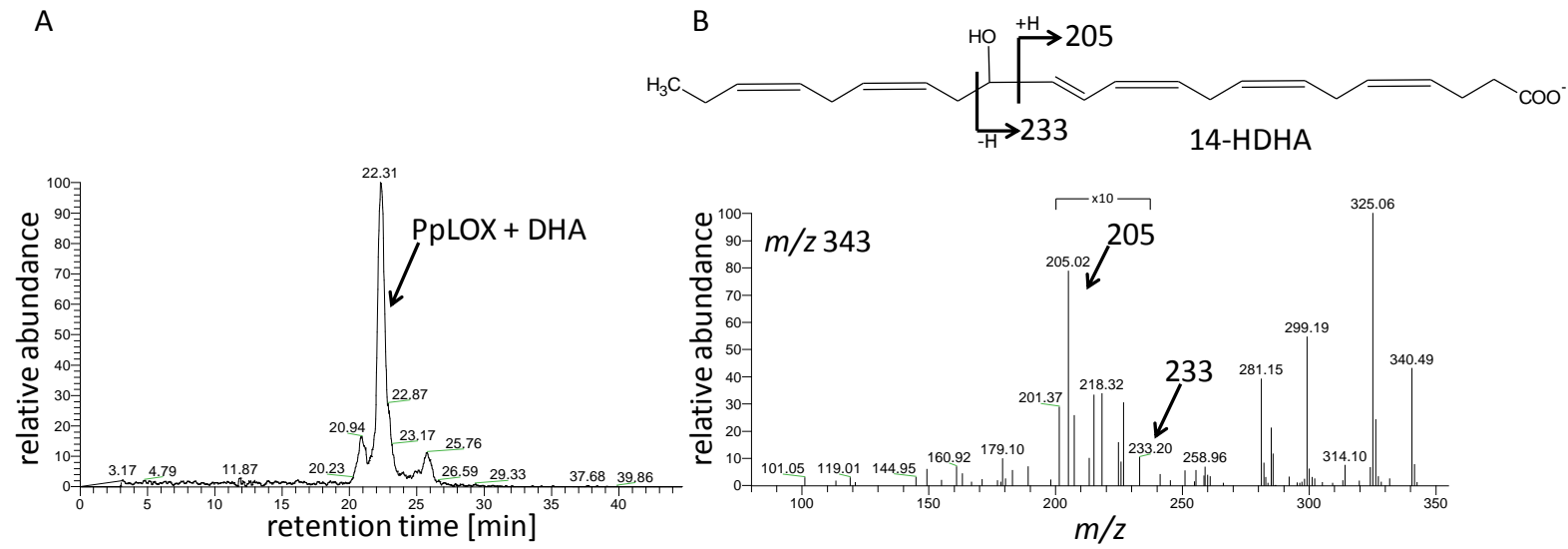

Figure 9.9: RP-HPLC/MS ${ }^{2}$-analysis of products formed by incubation of PpLOX with DHA. Shown are the extracted ion chromatograms $\mathrm{m} / \mathrm{z} 343$ and the associated tandem mass spectrum of $14-\mathrm{HDHA}$. 


\section{List of abbreviations}

${ }^{\circ} \mathrm{C}$

${ }^{\circ}$

TM

$\mathrm{ADH}$

$A O C$

AOS

approx.

APS

ARA

CA

$C D$

cDNA

$\mathrm{cm}$

CnA

C-terminal

CYP

d

DAD

$\mathrm{ddH}_{2} \mathrm{O}$

DES

DHA

DNA

dNTP

DTT

E

e.g.

EA

EAS

E. coli

EDTA

EnA

12,13S-EOT

EPA

ESI

et al.

$\mathrm{EtOH}$

$g$

$\mathrm{g}$

h

$\mathrm{HCl}$

His

10-H(P)DHA

14-H(P)DHA

17-H(P)DHA

12-HPETE

9-H(P)OD
Degree Celsius

Registered trademark

Trademark

Alcohol dehydrogenase

Allene oxide cyclase

Allene oxide synthase

approximately

Ammonium persulfate

Arachidonic acid

Colneleic acid

Circular Dichroism

complementary deoxyribonucleic acid

Centimeter

Colnelenic acid

Carboxy terminal

Cytochrome P

Day

Diode array detector

Double distilled water

Divinylether synthase

Docosahexaenoic acid

Deoxyribonucleic acid

Deoxynucleotidetriphosphate

Dithiothreitol

trans configuration

exempli gratia (for example)

Etheroleic acid

Epoxyalcohole synthase

Escherichia coli

Ethylenediaminetetraacetic acid

Etherolenic acid

(9Z,11E,13S,15Z)-12,13-epoxy-9,11,15-octadecatrienoic acid

Eicosapentaenoic acid

Electro spray ionization

et alii, et aliae; and others

Ethanol

gravitation acceleration

Gram

Hour

Hydrochloric acid

Histidine

(4Z,7Z,11E,13Z,16Z,19Z)-10-hydro(pero)xy-4,7,11,13,15,19docosahexaenoic acid

(4Z,7Z,10Z,12E,16Z,19Z)-14-hydro(pero)xy-4,7,11,13,15,19docosahexaenoic acid

(4Z,7Z,10Z,13Z,15E,19Z)-17-hydro(pero)xy-4,7,11,13,15,19docosahexaenoic acid

(5Z,8Z,10E,14Z)-12-hydroperoxy-5,8,10,14-eicosatetraenoic acid

(9S,10E,12Z)-9-hydro(pero)xy-10,12-octadecadienoic acid 


\begin{tabular}{|c|c|}
\hline $9 \alpha-H(P) O T$ & $\begin{array}{l}(9 S, 10 E, 12 Z, 15 Z)-9-h y d r o(p e r o) x y-10,12,15 \text {-octadecatrienoic } \\
\text { acid }\end{array}$ \\
\hline $9 \gamma-\mathrm{H}(\mathrm{P}) \mathrm{OT}$ & $\begin{array}{l}\text { (9S,6Z,10E,12Z)-9-hydro(pero)xy-6,10,12-octadecatrienoic } \\
\text { acid }\end{array}$ \\
\hline $13-H(P) O D$ & $(9 Z, 11 E, 13 S)$-13-hydro(pero)xy-9,11-octadecadienoic acid \\
\hline $13 \alpha-H(P) O T$ & $\begin{array}{l}(9 Z, 11 E, 13 S, 15 Z)-13 \text {-hydro(pero)xy-9,11,15-octadecatrienoic } \\
\text { acid }\end{array}$ \\
\hline $13 \gamma-\mathrm{H}(\mathrm{P}) \mathrm{OT}$ & $\begin{array}{l}(6 Z, 9 Z, 11 E, 13 S,)-13 \text {-hydro(pero)xy-6,9,11-octadecatrienoic } \\
\text { acid }\end{array}$ \\
\hline HPL & Hydroperoxide lyase \\
\hline HPLC & High Performance Liquid Chhromatography \\
\hline IMAC & Immobilized Metal Affinity Chromatography \\
\hline inter alia & among other things \\
\hline IPTG & Isopropyl $\beta$-D-thiogalactopyranoside \\
\hline$J A$ & Jasmonic acid \\
\hline KAc & Calium acetate \\
\hline $\mathrm{kb}$ & Kilobasepair \\
\hline $\mathrm{k}_{\text {cat }}$ & turnover number \\
\hline kDa & Kilo Dalton \\
\hline$K_{M}$ & Michaelis constant \\
\hline KOD & Ketodiene/Keto octadecadienoic acid \\
\hline KOT & Ketotriene/Keto octadecatrienoic acid \\
\hline kV & Kilovolt \\
\hline $\mathrm{L}$ & Liter \\
\hline LA & Linoleic acid \\
\hline LB & Luria-Bertani \\
\hline$\alpha$-LeA & Alpha-linolenic acid \\
\hline$\gamma$-LeA & Gamma-linolenic acid \\
\hline $\log$ & Decadic logarithm \\
\hline LOX & Lipoxygenase \\
\hline $\mathrm{M}$ & Molar (mole per liter) \\
\hline $\mathrm{mA}$ & Milliampere \\
\hline $\mathrm{mg}$ & Milligram \\
\hline MgAc & Magnesium acetate \\
\hline $\mathrm{MgCl}_{2}$ & Magnesium chloride \\
\hline $\min$ & Minute \\
\hline $\mathrm{ml}$ & Milliliter \\
\hline $\mathrm{mM}$ & Millimol per liter \\
\hline MS & Mass spectrometry \\
\hline$m / z$ & Ratio of mass to charge \\
\hline$\mu$ & Micro \\
\hline$\mu g$ & Microgram \\
\hline$\mu \mathrm{L}$ & Microliter \\
\hline$\mu \mathrm{m}$ & Micrometer \\
\hline$\mu \mathrm{M}$ & Micromol pro Liter \\
\hline$\mu \mathrm{mol}$ & Micromol \\
\hline $\mathrm{NaAc}$ & Sodium acetate \\
\hline $\mathrm{NaCl}$ & Sodium chloride \\
\hline $\mathrm{NAD}(\mathrm{P}) \mathrm{H}$ & Nicotinamide adenine dinucleotide phosphate \\
\hline n. d. & Not detected \\
\hline ng & Nanogram \\
\hline $\mathrm{nm}$ & Nanometer \\
\hline $\mathrm{nM}$ & Nanomol \\
\hline
\end{tabular}




\begin{tabular}{|c|c|}
\hline N-terminal & Amino-terminus \\
\hline$O D_{x}$ & Optical density at a wavelenght of $\mathrm{X} \mathrm{nm}$ \\
\hline 12-OPDA & $(10 Z, 15 Z)-12-o x o-10,15-p h y t o d i e n o i c ~ a c i d$ \\
\hline OPR3 & 12-oxo-phytodienoic acid-reductase isoform 3 \\
\hline PAGE & PolyÁcrylamide GelElektrophoresis \\
\hline PCR & Polymerase $\underline{\text { Chain }}$ Reaction \\
\hline $\mathrm{pH}$ & Negative decadic logarithm of hydrogen ion activity \\
\hline pmol & pico Mol \\
\hline PMSF & Phenylmethanesulfonyl fluoride \\
\hline P. patens & Physcomitrella patens \\
\hline PUFA & 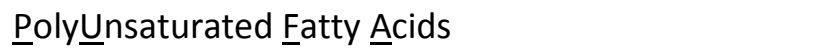 \\
\hline PXG & Peroxygenase \\
\hline $\mathrm{RP}$ & 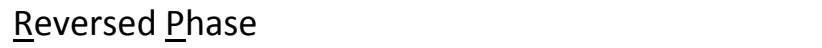 \\
\hline rpm & rotations per minute \\
\hline RT & Room temperature \\
\hline S & Second \\
\hline SDS & Sodium Dodecyl $\underline{\text { Sulfate }}$ \\
\hline SP & Straight $\underline{P h a s e}$ \\
\hline TAE & Tris-acetate-EDTA \\
\hline TEMED & N,N,N,N-tetramethylenediamine \\
\hline TFA & Trifluoroacetic acid \\
\hline Tris & 2-Amino-2-hydroxymethyl-propane-1,3diol \\
\hline UV & 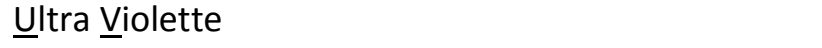 \\
\hline v & reaction rate \\
\hline V & Volt \\
\hline Vol & Volume \\
\hline vis & visible \\
\hline $\mathrm{V}_{\max }$ & Maximum reaction rate \\
\hline $\mathrm{v} / \mathrm{v}$ & Volume per volume \\
\hline$w / v$ & Weight per volume \\
\hline Wt & Wild type \\
\hline YT & Yeast Extract Tryptone \\
\hline$Z$ & cis configuration \\
\hline
\end{tabular}




\section{List of figures}

Figure 1.1: Synthesis of hydroperoxides derived from LA by 9-LOX or 13-LOX (adapted from (Stumpe and Feussner, 2006)).

Figure 1.2: Formation of oxylipins in plants (adapted from (Göbel et al., 2001)).....

Figure 1.3: Phylogenetic analysis of different CYP74 enzymes from different plant species (taken from (Scholz et al., 2012)). 5

Figure 1.4: AOS pathway for the conversion of 13-HPOT (adapted from (Stumpe and Feussner, 2006)).

Figure 1.5: HPL pathway for the conversion of $\alpha$-LeA (adapted from (Stumpe and Feussner, 2006))... 8

Figure 1.6: DES pathway for the conversion of $\alpha$-LeA (adapted from (Hamberg, 2004)). 9

Figure 1.7: EAS pathway for the conversion of $\alpha$-LeA 10

Figure 1.8: Proposed reaction mechanisms for CYP74-enzymes (adapted from (Schaller and Stintzi, 2009)).

Figure 1.9: Proposed potential intermediates in radical and ionic pathways to CYP74 products (adapted from (Brash, 2009)).

Figure 3.1: SDS-PAGE analysis of PpAOS1 purification by stepwise elution. 35

Figure 3.2: SDS-PAGE analysis of PpAOS1 purification by gradual elution.. 36

Figure 3.3: SDS-PAGE analysis of PpHPL (A, B) and PpAOS2 (C) purification. 37

Figure 3.4: UV/vis spectra of purified PpAOS1 (A), PpAOS2 (B) and PpHPL (C). 39

Figure 3.5: CD spectrum of purified PpHPL 40

Figure 3.6: $\mathrm{CD}$ spectrum of purified PpAOS1. 40

Figure 3.7: Product analysis of PpAOS1 and PpHPL with $\left[1-{ }^{14} \mathrm{C}\right]-9-\mathrm{HPOD}$. 42

Figure 3.8: Product analysis of PpAOS1 and PpHPL with $\left[1-{ }^{14} \mathrm{C}\right]-13 \alpha-\mathrm{HPOT}$. 43

Figure 3.9: Product analysis of StDES with $\left[1-{ }^{14} \mathrm{C}\right]-9 \alpha-\mathrm{HPOT}$. 44 
Figure 3.10: RP-HPLC/MS ${ }^{2}$-analysis of products formed by incubation of PpAOS1 with $13 \alpha$-HPOT ( $\gamma$ ketol). .45

Figure 3.11: RP-HPLC/MS ${ }^{2}$-analysis of products formed by incubation of PpAOS1 with $13 \alpha$-HPOT ( $\alpha$ ketol). 45

Figure 3.12: RP-HPLC/MS ${ }^{2}$-analysis of products formed by incubation of PpAOS1 with $13 \alpha$-HPOT (cyclopentenone).

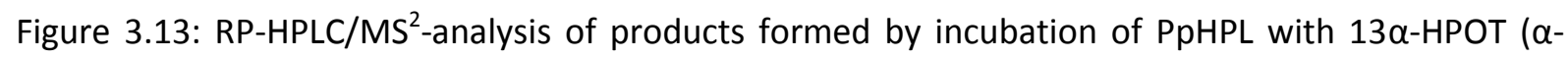
and $\gamma$-ketol).

Figure 3.14: The pH optimum of PpAOS1 with 13-HPOD...

Figure 3.15: Kinetic analyses of PpAOS1 with different hydroperoxides from $C_{18}$ fatty acids as substrates.

Figure 3.16: Kinetic analysis of PpAOS1 with 12-HPETE. 50

Figure 3.17: Partial multiple sequence alignment of different CYP74-enzymes from different plant species and marine invertebrates. 51

Figure 3.18: Partial multiple sequence alignment of different CYP74-enzymes from different plant species and marine invertebrates.

Figure 3.19: UV/vis spectra of purified PpHPL_F151L (A), PpHPL_A169S (B), and PpHPL_F151L, A169S

(C) in comparison to the wild type (D).

Figure 3.20: $C D$ sepcra of purified variants from PpHPL in comparison to the wild type. 54

Figure 3.21: Product analysis of PpAOS_F93L with $\left[1-{ }^{14} \mathrm{C}\right]-9-\mathrm{HPOD}$ in comparison to the PpAOS1 and PpHPL wild types. 55

Figure 3.22: Product analysis of PpAOS_F93L with $\left[1-{ }^{14} \mathrm{C}\right]-13 \alpha-\mathrm{HPOT}$ in comparison to the PpAOS1 and PpHPL wild types. 56

Figure 3.23: SDS-PAGE analyses of BfEAS purified from different $E$. coli BL21 Star cultivations $\left(11^{\circ} \mathrm{C}\right.$ or at $28^{\circ} \mathrm{C}$ for $\left.1 \mathrm{~d}\right)$. 58

Figure 3.24: SDS-PAGE of purified BfEAS expression at $16^{\circ} \mathrm{C}$ for $3 \mathrm{~d}$ 59

Figure 3.25: UV/vis spectrum of purified BfEAS in $50 \mathrm{mM}$ sodium phosphate buffer, $\mathrm{pH}$ 8.0. 60

Figure 3.26: $C D$ sepctrum of purified BfEAS. 
Figure 3.27: Structure of $\omega-3$ fatty acid docosahexaenoic acid (DHA) and its possible ten positions that may be oxidized enzymes.

Figure 3.28: Product analysis from incubation of BfEAS with $\left[1-{ }^{14} \mathrm{C}\right]-9 \alpha-\mathrm{HPOT}$. 62

Figure 3.29: Product analysis from incubation of BfEAS with $\left[1-{ }^{14} \mathrm{C}\right]-13 \alpha-\mathrm{HPOT}$. 63

Figure 3.30: RP-HPLC/MS ${ }^{2}$-analysis of products formed by incubation of BfEAS with $9 \alpha-\mathrm{HPOT}$. 64

Figure 3.31: RP-HPLC/MS ${ }^{2}$-analysis of products formed by incubation of BfEAS with $9 \alpha$-HPOT (divinyl ether). 65

Figure 3.32: RP-HPLC/MS²-analysis of products formed by incubation of BfEAS with $13 \alpha-H P O T$. 65

Figure 3.33: RP-HPLC/MS ${ }^{2}$-analysis of products formed by incubation of GmLOX1 with DHA. 66

Figure 3.34: RP-HPLC/MS ${ }^{2}$-analysis of products formed by incubation of GmLOX1 with DHA and BfEAS.

Figure 3.35: RP-HPLC/MS ${ }^{2}$-analysis of products formed by incubation of AtLOX1 with DHA and BfEAS.

Figure 3.36: RP-HPLC/MS ${ }^{2}$-analysis of products formed by incubation of StLOX with DHA and BfEAS.

Figure 3.37: RP-HPLC/MS²-analysis of products formed by incubation of PpLOX with DHA and BfEAS. 68

Figure 3.38: The pH optimum of BfEAS with 13-HPOD. 69

Figure 3.39: Kinetic analyses of BfEAS with 13-HPOD (A) and $13 \alpha-$ HPOT (B). 70

Figure 3.40: Kinetic analysis of BfEAS with 17-HPDHA.

Figure 3.41: Partial multiple sequence alignment of different CYP74 enzymes from different plant species and marine invertebrates. 72

Figure 3.42: Partial multiple sequence alignment of different CYP74 enzymes from different plant species and marine invertebrates. 
Figure 3.44: UV/vis spectra of purified BfEAS_F58L (A) and BfEAS_9AS-deletion (B) in comparison to the BfEAS wild type (C). .75

Figure 3.45: CD sepcra of purified BfEAS_F58L (A) and BfEAS_9AS-deletion (B) in comparison to BfEAS wild type (C).....

Figure 3.46: Product analysis from incubation of BfEAS, BfEAS_F58L, and BfEAS_9AS-deletion with [1$\left.{ }^{14} \mathrm{C}\right]-9 \alpha$-HPOT. 77

Figure 3.47: Product analysis from incubation of BfEAS, BfEAS_F58L, and BfEAS_9AS-deletion with [1$\left.{ }^{14} \mathrm{C}\right]-13 \alpha-$-HPOT. .78

Figure 3.48: RP-HPLC/MS ${ }^{2}$-analysis of products formed by incubation of BfEAS_F58L with $9 \alpha$-HPOT in comparison to the wild type (insertion). 80

Figure 3.49: RP-HPLC/MS²-analysis of products formed by incubation of BfEAS_F58L with 13 $\alpha$-HPOT in comparison to the wild type (insertion).

Figure 9.1: Product analyses from incubation of PpAOS1 and PpHPL with $\left[1-{ }^{14} \mathrm{C}\right]-9 \alpha-\mathrm{HPOT}$ (A) and with $\left[1-{ }^{14} \mathrm{C}\right]-13-\mathrm{HPOD}(\mathrm{B})$. 116

Figure 9.2: Product analyses of PpAOS_F93L with $\left[1-{ }^{14} \mathrm{C}\right]-9 \alpha-\mathrm{HPOT}(\mathrm{A})$ and with $\left[1-{ }^{14} \mathrm{C}\right]-13-\mathrm{HPOD}(\mathrm{B})$ in comparison to the PpAOS1 and PpHPL wild types. 116

Figure 9.3: Product analyses from incubation of BfEAS with $\left[1-{ }^{14} \mathrm{C}\right]-9-\mathrm{HPOD}(\mathrm{A})$ and with $\left[1-{ }^{14} \mathrm{C}\right]-13-$ HPOD (B).

Figure 9.4: Product analyses from incubation of BfEAS_F58L and BfEAS_9AS-deletion with $\left[1-{ }^{14} \mathrm{C}\right]-9-$ HPOD (A) and with $\left[1-{ }^{14} \mathrm{C}\right]-13-\mathrm{HPOD}(\mathrm{B})$ in comparison to BfEAS wild type.

Figure 9.5: RP-HPLC/MS²-analysis of products formed by incubation of BfEAS with 9-HPOD. 118

Figure 9.6: RP-HPLC/MS'-analysis of products formed by incubation of BfEAS with 13-HPOD. 118

Figure 9.7: RP-HPLC/MS ${ }^{2}$-analysis of products formed by incubation of AtLOX1 with DHA. 118

Figure 9.8: RP-HPLC/MS'-analysis of products formed by incubation of StLOX with DHA. 119

Figure 9.9: RP-HPLC/MS'-analysis of products formed by incubation of PpLOX with DHA. 119 


\section{List of tables}

Table 2.1: Bacterial strains used

Table 2.2: Vectors used.

Table 2.3: Flow diagram for the RP-HPLC separation.

Table 2.4: Flow diagram for the radio-RP-HPLC separation. 30

Table 2.5: Flow diagram for the RP-HPLC/MS ${ }^{2}$ separation. 31

Table 2.6: Range $\mathrm{pH}$ and buffer used for measurements of PpAOS1.

Table 2.7: Range $\mathrm{pH}$ and buffer used for measurements of BfEAS.

Table 3.1: Expression vectors used in this study.

Table 3.2: Product specificities of PpHPL and PpAOS1.

Table 3.3: Kinetic parameters for the reaction of PpAOS1 with different hydroperoxides from $\mathrm{C}_{18}$ - and $\mathrm{C}_{20}$ fatty acids.. 50

Table 3.4: Product specificities of different PpHPL and PpAOS1 variants. 57

Table 3.5: Product specificities of BfEAS. 64

Table 3.6: Product formation of conversions by various LOXs with DHA. 66

Table 3.7: Kinetic parameters for the reaction of BfEAS with different hydroperoxides from $\mathrm{C}_{18^{-}}$and $\mathrm{C}_{22}$ fatty acids...

Table 3.8: Product specificities of BfEAS wild type and various BfEAS variants. 79

Table 4.1: Re-evaluation of the kinetic parameters for the reaction of PpAOS2 with different hydroperoxides derived from $\mathrm{C}_{18}$ and $\mathrm{C}_{20}$ fatty acids...

Table 4.2: Products formed by conversions of 9-and 13-hydroperoxides derived from $\mathrm{C}_{18}$ fatty acids LA and $\alpha$-LeA by BfEAS.

Table 4.3: Products formed by conversions of 10-, 14-, and 17-hydroperoxides derived from $C_{22}$ fatty acid DHA by BfEAS. 


\section{Publications}

Scholz, J., Brodhun, F., Hornung, E., Herrfurth, C., Stumpe, M., Beike, A., Faltin, B., Frank, W., Reski, R. \& Feussner, I. (2012). Biosynthesis of allene oxides in Physcomitrella patens. BMC Plant Biology, 12: 228.

Neumann, P., Brodhun, F., Sauer, K., Herrfurth, C., Hamberg, M., Brinkmann, J., Scholz, J., Dickmanns, A., Feussner, I. \& Ficner, R. (2012). Crystal structures of Physcomitrella patens AOC1 and 2: Insights into the enzyme mechanism and differences in substrate specificity. Plant Physiol: doi: 10.1104/pp.112.205138. 